\title{
Perseverance's Scanning Habitable Environments with Raman and Luminescence for Organics and Chemicals (SHERLOC) Investigation
}

Rohit Bhartia $^{1}$ - Luther W. Beegle ${ }^{2}$ (D) - Lauren DeFlores ${ }^{2} \cdot$ William Abbey $^{2}$. Joseph Razzell Hollis ${ }^{2} \cdot$ Kyle Uckert $^{2}$ - Brian Monacelli ${ }^{2} \cdot$ Kenneth S. Edgett $^{3}$. Megan R. Kennedy ${ }^{3} \cdot$ Margarite Sylvia $^{2}$ - David Aldrich ${ }^{2} \cdot$ Mark Anderson $^{2}$. Sanford A. Asher ${ }^{4}$ - Zachary Bailey ${ }^{2}$ Kerry Boyd ${ }^{5}$. Aaron S. Burton ${ }^{6}$. Michael Caffrey ${ }^{5}$ Michael J. Calaway ${ }^{7}$ - Robert Calvet ${ }^{2} \cdot$ Bruce Cameron $^{2}$. Michael A. Caplinger ${ }^{3}$ - Brandi L. Carrier ${ }^{2}$ - Nataly Chen² - Amy Chen ${ }^{2}$. Matthew J. Clark ${ }^{3} \cdot$ Samuel Clegg $^{5} \cdot$ Pamela G. Conrad $^{8} \cdot$ Moogega Cooper $^{2}$. Kristine N. Davis ${ }^{6}$ - Bethany Ehlmann' $\cdot$ Linda Facto ${ }^{2}$ - Marc D. Fries ${ }^{6}$. Dan H. Garrison ${ }^{7}$ - Denine Gasway ${ }^{5}$ - F. Tony Ghaemi ${ }^{10}$. Trevor G. Graff ${ }^{7}$. Kevin P. Hand ${ }^{2}$ - Cathleen Harris ${ }^{2}$. Jeffrey D. Hein ${ }^{2}$ - Nicholas Heinz ${ }^{2}$. Harrison Herzog 2 • Eric Hochberg ${ }^{2}$ • Andrew Houck ${ }^{2}$ • William F. Hug ${ }^{1}$. Elsa H. Jensen ${ }^{3} \cdot$ Linda C. Kah $^{11} \cdot$ John Kennedy $^{2} \cdot$ Robert Krylo $^{2} \cdot$ Johnathan Lam $^{2}$ • Mark Lindeman ${ }^{2} \cdot$ Justin McGlown $^{5} \cdot$ John Michel $^{5} \cdot$ Ed Miller $^{2} \cdot$ Zachary Mills $^{2}$. Michelle E. Minitti' ${ }^{12}$ - Fai Mok $^{2}$ - James Moore ${ }^{2} \cdot K_{\text {Kenneth H. Nealson }}{ }^{13}$. Anthony Nelson ${ }^{5}$ - Raymond Newell ${ }^{5}$ Brian E. Nixon ${ }^{3}$. Daniel A. Nordman ${ }^{2}$. Danielle Nuding $^{14}$ - Sonny Orellana ${ }^{2}$. Michael Pauken ${ }^{2}$. Glen Peterson ${ }^{5}$. Randy Pollock ${ }^{2}$ Heather Quinn ${ }^{5}$. Claire Quinto ${ }^{2}$. Michael A. Ravine ${ }^{3}$. Ray D. Reid ${ }^{1} \cdot$ Joe Riendeau $^{2}$ • Amy J. Ross ${ }^{6}$. Joshua Sackos ${ }^{5}$. Jacob A. Schaffner ${ }^{3}$. Mark Schwochert ${ }^{2} \cdot$ Molly O Shelton ${ }^{2}$ Rufus Simon ${ }^{2}$. Caroline L. Smith ${ }^{15}$. Pablo Sobron ${ }^{16}$ - Kimberly Steadman ${ }^{2}$. Andrew Steele ${ }^{8}$. Dave Thiessen ${ }^{2}$. Vinh D. Tran ${ }^{7}$. Tony Tsai ${ }^{2} \cdot$ Michael Tuite $^{2}$ Eric Tung ${ }^{2} \cdot$ Rami Wehbe $^{2}$. Rachel Weinberg ${ }^{2} \cdot$ Ryan H. Weiner $^{7} \cdot$ Roger C. Wiens $^{5} \cdot$ Kenneth Williford $^{2}$.

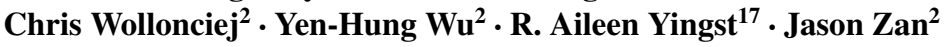

The Mars 2020 Mission

Edited by Kenneth A. Farley, Kenneth H. Williford and Kathryn M. Stack

\section{L.W. Beegle}

1 Photon Systems Inc., 1512 Industrial Park St., Covina, CA 91722, USA

2 Jet Propulsion Laboratory, California Institution of Technology, 4800 Oak Grove Drive, Pasadena, CA 91109, USA

3 Malin Space Science Systems, Inc., San Diego, CA, USA

4 Pittsburg University, Pittsburg, PA, USA

5 Los Alamos National Laboratory, Los Alamos, NM 87545, USA 


\begin{abstract}
The Scanning Habitable Environments with Raman and Luminescence for Organics and Chemicals (SHERLOC) is a robotic arm-mounted instrument on NASA's Perseverance rover. SHERLOC has two primary boresights. The Spectroscopy boresight generates spatially resolved chemical maps using fluorescence and Raman spectroscopy coupled to microscopic images $(10.1 \mu \mathrm{m} / \mathrm{pixel})$. The second boresight is a Wide Angle Topographic Sensor for Operations and eNgineering (WATSON); a copy of the Mars Science Laboratory (MSL) Mars Hand Lens Imager (MAHLI) that obtains color images from microscopic scales $(\sim 13 \mu \mathrm{m} /$ pixel) to infinity. SHERLOC Spectroscopy focuses a $40 \mu$ s pulsed deep UV neon-copper laser $(248.6 \mathrm{~nm})$, to a $\sim 100 \mu \mathrm{m}$ spot on a target at a working distance of $\sim 48$ $\mathrm{mm}$. Fluorescence emissions from organics, and Raman scattered photons from organics and minerals, are spectrally resolved with a single diffractive grating spectrograph with a spectral range of 250 to $\sim 370 \mathrm{~nm}$. Because the fluorescence and Raman regions are naturally separated with deep UV excitation $(<250 \mathrm{~nm})$, the Raman region $\sim 800-4000 \mathrm{~cm}^{-1}$ ( 250 to $273 \mathrm{~nm}$ ) and the fluorescence region ( 274 to $\sim 370 \mathrm{~nm}$ ) are acquired simultaneously without time gating or additional mechanisms. SHERLOC science begins by using an Autofocus Context Imager (ACI) to obtain target focus and acquire $10.1 \mu \mathrm{m} /$ pixel greyscale images. Chemical maps of organic and mineral signatures are acquired by the orchestration of an internal scanning mirror that moves the focused laser spot across discrete points on the target surface where spectra are captured on the spectrometer detector. ACI images and chemical maps ( $<100 \mu \mathrm{m} /$ mapping pixel) will enable the first Mars in situ view of the spatial distribution and interaction between organics, minerals, and chemicals important to the assessment of potential biogenicity (containing CHNOPS). Single robotic arm placement chemical maps can cover areas up to $7 \mathrm{x} 7 \mathrm{~mm}$ in area and, with the $<10$ min acquisition time per map, larger mosaics are possible with arm movements. This microscopic view of the organic geochemistry of a target at the Perseverance field site, when combined with the other instruments, such as Mastcam-Z, PIXL, and SuperCam, will enable unprecedented analysis of geological materials for both scientific research and determination of which samples to collect and cache for Mars sample return.
\end{abstract}

\title{
Acronyms
}

ACI Autofocus and Contextual Imager

ADA Adaptive Caching Assembly

ACA Adaptive Caching Assembly

6 NASA Johnson Space Center, Houston, TX 77058, USA

7 Jacobs Engineering, NASA Jonson Space Center, Houston, TX 77058, USA

8 Carnegie Insitutie of Washington, Washington, DC, USA

9 California Institution of Technology, Pasadena, CA, USA

10 Ghaemi Optical Engineering, San Diego, CA, USA

11 University of Tennessee, Knoxville, TN, USA

12 Framework, Silver Spring, MD, USA

13 University of Southern California, Los Angeles, CA, USA

14 Applied Physics Laboratory, Johns Hopkins University, Baltimore, MD, USA

15 Natural History Museum, London, UK

16 SETI Institute, Mountain View, CA, USA

17

Planetary Science Institute, Tucson, AZ, USA 


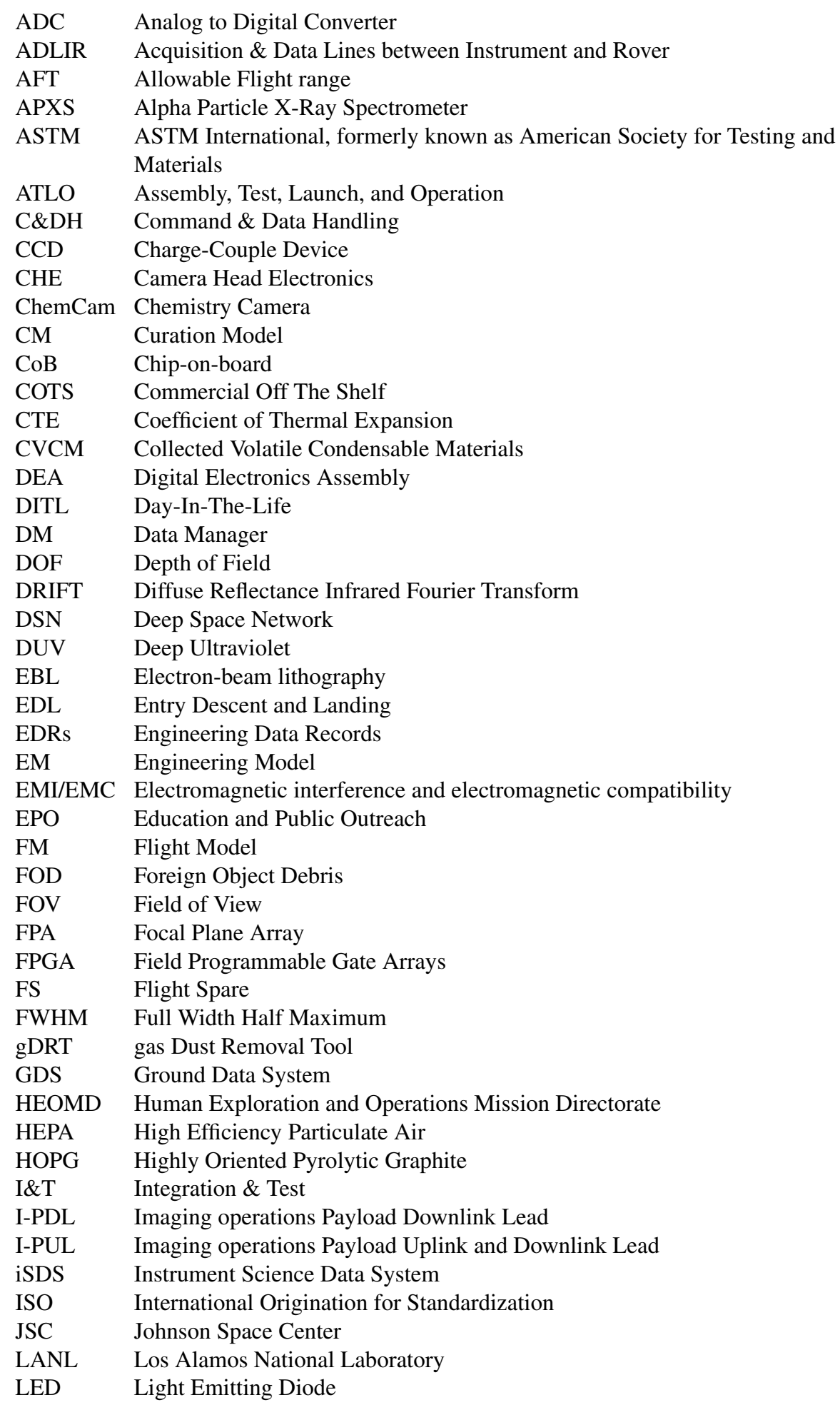




\begin{tabular}{|c|c|}
\hline LIF & Laser Injection Filter \\
\hline LPF & Long-Pass Filter \\
\hline LPS & Laser Power Supply \\
\hline LVDS & Low-Voltage Differential Signal \\
\hline M2020 & Mars 2020 mission \\
\hline MAHLI & Mars Hand Lens Imager \\
\hline MDL & Micro-Devices Laboratory \\
\hline MEDA & Mars Environmental Dynamics Analyzer \\
\hline MER & Mars Exploration Rover \\
\hline MI & Microscopic Imager \\
\hline MMRTG & Multi-Mission Radioisotope Thermoelectric Generator \\
\hline MSL & Mars Science Laboratory \\
\hline MSSS & Malin Space Science Systems \\
\hline MTF & modulation transfer function \\
\hline MUX & Multiplexer \\
\hline NASA & National Aeronautics and Space Administration \\
\hline NIST & National Institute of Standards and Technology \\
\hline NIR & Near infrared \\
\hline NVM & Non-Volatile memory \\
\hline NVR & Non-Volatile Residue \\
\hline OAP & Off-Axis Paraboloidal \\
\hline OFHC & Oxygen-Free, High thermal Conductivity \\
\hline PAHs & Polycyclic Aromatic Hydrocarbons \\
\hline PCB & Printed Circuit Board \\
\hline PCM & Phase Change Material \\
\hline PDL & Payload Downlink Lead \\
\hline PDS & Planetary Data System \\
\hline PDU & Power Distribution Unit \\
\hline PGE & Product Generated Executable \\
\hline PIXL & Planetary Instrument for X-ray Lithochemistry \\
\hline PQV & Package Qualification and Verification \\
\hline PRT & platinum resistance thermometer \\
\hline PSFs & Point Spread Functions \\
\hline PSI & Photon Systems Inc. \\
\hline PUL & Payload Uplink Lead \\
\hline QE & Quantum efficiency \\
\hline QTH & Quartz Tungsten Halogen \\
\hline QRM & Quantitative Relief Model \\
\hline RAMP & Rover Avionics Mounting Plate \\
\hline RCE & Rover computing elements \\
\hline RDRs & Reduced Data Records \\
\hline RGB & Red, Green and Blue \\
\hline RMI & Remote Microscopic Imager \\
\hline ROI & Region of Interest \\
\hline RPAM & Rover Power Avionics Module \\
\hline SAM & Sample Analysis at Mars \\
\hline SaU 008 & Sayh al Uhaymir 008 \\
\hline SBA & SHERLOC Body Assembly \\
\hline SCCD & Spectrometer Charge-Couple Device \\
\hline
\end{tabular}




$\begin{array}{ll}\text { Sc-PDL } & \text { SHERLOC Science Payload Downlink Lead } \\ \text { Sc-PUL } & \text { SHERLOC Science Payload Uplink Lead } \\ \text { SCT } & \text { SHERLOC Calibration Target } \\ \text { SDE } & \text { Scanner Demodulator Electronics } \\ \text { SE } & \text { Spectrometer Electronics } \\ \text { SfM } & \text { Structure from Motion } \\ \text { SHERLOC } & \text { Scanning Habitable Environments for Raman and Luminescence for Organics } \\ & \text { and Chemicals } \\ \text { SOL } & \text { SHERLOC Operations Lead } \\ \text { Sp-PDL } & \text { Spectroscopy operations Payload Downlink Lead } \\ \text { Sp-PUL } & \text { Spectroscopy operations Payload Uplink and Downlink Lead } \\ \text { SSE } & \text { Scanner Servo Electronics } \\ \text { STA } & \text { SHERLOC Turret Assembly } \\ \text { STP } & \text { Space Technology Program } \\ \text { STT } & \text { System Thermal Test } \\ \text { TAYF } & \text { Test As You Fly } \\ \text { TIG } & \text { Tungsten Inert Gas } \\ \text { TML } & \text { Total Mass Loss } \\ \text { TPRB } & \text { Turret Power Regulation Board } \\ \text { TQCM } & \text { Thermoelectric Quartz Crystal Microbalance, } \\ \text { TRCE } & \text { Temperature cycling resistant electronics } \\ \text { UV } & \text { Ultraviolet } \\ \text { VIS } & \text { Human-visible portion of electromagnetic spectrum } \\ \text { VM } & \text { Volatile memory } \\ \text { WATSON } & \text { Wide Angle Telegraphic Sensor for Operations and eNgineering } \\ \text { WCHE } & \text { WATSON Camera Head Electronics } \\ \text { WEB } & \text { Warm Electronics Box }\end{array}$

\section{Introduction}

Since ancient times, Mars has been at the forefront of astronomical observations as we struggle to understand our place in the universe. It was named for the god of war and was once thought to be a harbinger of death and plague. Telescopes trained on Mars by such observers as Giovanni Schiaparelli and Percival Lowell led imaginations to popularly envision an inhabited and vegetated Mars. However, with more advanced telescopic observations and dawn of the space age, it was clear that Mars has a cold, dry environment that appeared to be devoid of life. As we continued to explore Mars it was obvious from orbiter and rover observations that the Mars of the past was a very different place. All indications, including results from NASA's Mars Science Laboratory (MSL; Curiosity rover), indicate that Mars had periods of stable liquid water on its surface and that habitable environments existed during a significant period of its history (e.g. Carr and Head 2003; Perron et al. 2007; Grotzinger et al. 2014). Orbital and in situ observations show evidence of volcanism and hydrothermal environments that could provide energy for life (Squyres et al. 2007; Ruff et al. 2020). Mars also had access to organic carbon through both in situ synthesis at hydrothermal vents and through meteoritic infall (Schock and Schulte 1998; Flynn 1996; Benner et al. 2000; Cady et al. 2018). All of this occurred at a time when life started on Earth, leading astrobiologists to ask the question, did life also originate on Mars? And, if not, why not? 


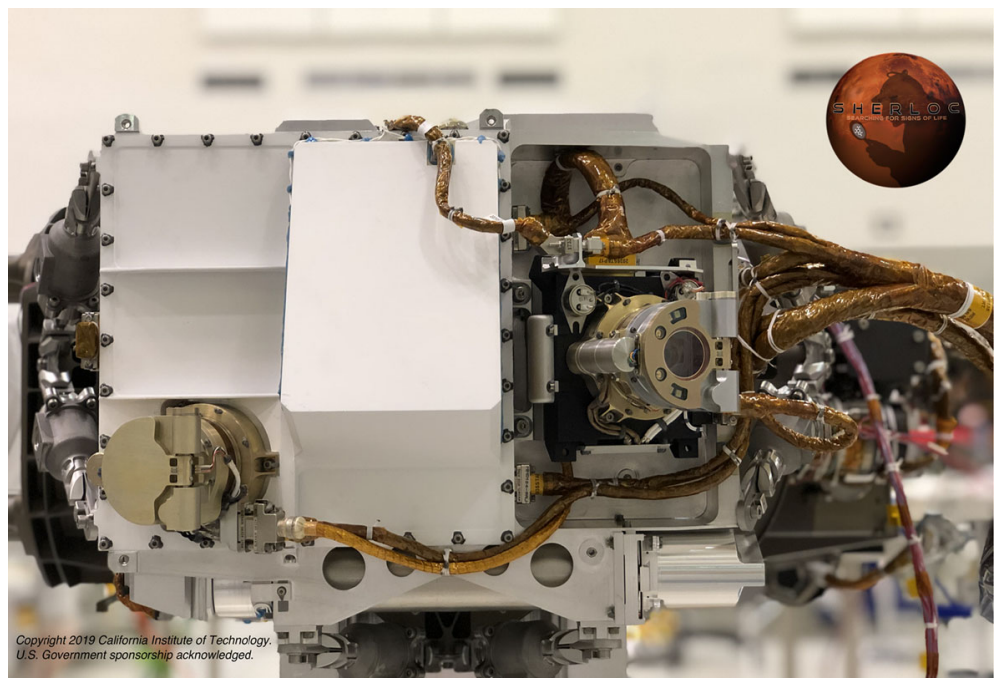

Fig. 1 The SHERLOC Turret Assembly (STA) on the Perseverance robotic arm during Assembly, Test, and Launch Operations (ATLO) testing. Both the spectroscopy/ACI boresight (Left) and WATSON boresight (Right) are visible

To address these questions, understand its potential habitability, and to look for evidence of past life, NASA launched the Perseverance rover to Mars in 2020 (Farley et al. 2020). The Mars 2020 mission has four science goals:

A. Explore an astrobiologically relevant ancient environment on Mars to decipher its geological processes and history, including the assessment of past habitability.

B. Assess the biosignature preservation potential within the selected geological environment and search for potential biosignatures.

C. Demonstrate significant technical progress towards the future return of scientifically selected, well-documented samples to Earth.

D. Provide an opportunity for contributed Human Exploration and Operations Mission Directorate (HEOMD) or Space Technology Program (STP) participation, compatible with the science payload and within the mission's payload capacity.

\subsection{SHERLOC Overview}

SHERLOC (Scanning Habitable Environments for Raman and Luminescence for Organics and Chemicals) is a robotic arm-mounted instrument onboard the Perseverance rover that will operate in Jezero crater on Mars (Fig. 1). SHERLOC enables high-sensitivity detection, characterization, and spatially resolved correlation of trace organics/biosignatures and minerals in geological materials at the Martian surface and near sub-surface (i.e. within boreholes). Using a deep ultraviolet (DUV) laser, SHERLOC obtains native fluorescence emissions from aromatic organic species and Raman scattered photons from molecules that allow identification of functional groups of organics, chemicals, and minerals. SHERLOC also acquires high spatial resolution color and gray scale images of natural and abraded samples on the surface in order to better understand the geologic history of Mars and help spatially correlate measurements made by SHERLOC and the other scientific instruments 
onboard Perseverance. SHERLOC's spectroscopic methodology enables a new science investigation for organic analysis on Mars where the fine-scale spatial distribution of organics within the context of minerals an be preserved to assess organic provenance; a capability previously relegated to laboratory instruments.

SHERLOC is designed to operate from the end of the rover's a robotic arm in a single arm deployment activity. Each spectral map obtained is approximately the size of a postage stamp. The robotic arm places SHERLOC over the target without surface contact, thus avoiding contamination of the instrument, rover contamination of the surface, or crosssample contamination. SHERLOC consists of a combined Raman and fluorescence spectrometer that analyzes photons generated through the interaction of a $248.6 \mathrm{~nm}$ pulsed laser beam with surface material. Perseverance will mark the first time that Raman spectroscopy will be attempted on the surface of another planet and will capitalize on the synergistic benefits of utilizing multi-wavelength Raman spectroscopy, with SHERLOC using a DUV excitation source and SuperCam (Maurice et al. 2021; Wiens et al. 2021) using a $532 \mathrm{~nm}$ source.

To simplify operations and avoid contacting the surface, several SHERLOC subsystems were included to aid the spectroscopy investigation. This includes an Autofocus Context Imager (ACI) for focusing the spectrometer at standoff distance of $\sim 50 \mathrm{~mm}$, an internal scanning mirror to avoid complex and sub-millimeter arm movements, and an optical design that focuses the laser to an $\sim 100 \mu \mathrm{m}$ beam on the target. In addition, both backscattered spectroscopic elements (Raman and fluorescence) are detected on the same CCD through a common optical path. The instrument has knowledge of where the laser was pointing within the ACI's grayscale image, thus providing an ability to correlate spectroscopic signals with the physical features of a target's surface. In addition, onboard spectral analysis enables autonomous retargeting of regions of interest for additional spectral measurements; providing an increased capability to locate minute, "needle-in-a-haystack" features of importance without multiple sols of planning and complex arm retargeting (a sol is a Martian day; duration 1.027 Earth days). All of these measurements are capable with a single, non-contact arm placement on either a natural surface or, preferably, an abraded surface for which dust and weathering products have been removed.

After selection of the initial Mars 2020 science payload, it was noted that there arose a need for an arm-mounted imager that could perform engineering tasks to document rover health, such as wheel imaging, rover undercarriage inspections, and Education and Public Outreach (EPO) activities. The ACI inherits many components derived from MSL's Mars Hand Lens Imager (MAHLI; Edgett et al. 2012; Grotzinger et al. 2012) hardware. A Mars 2020 Project-wide trade study was performed to determine the best way to integrate these needs within the rover payload. The options included modifying SHERLOC's ACI capabilities to generate MAHLI-like images. Given the spectroscopy-driven requirements on the ACI design, including a fixed focal length, it was infeasible to incorporate increased capability with the proposed hardware without significant complexity or reduction in spectroscopy performance. However, given the ACI's heritage based on MAHLI hardware, it was determined that the SHERLOC design could be modified to operate a second camera head, and hence the Wide Angle Telegraphic Sensor for Operations and eNgineering (WATSON) camera was integrated to the SHERLOC investigation. The shared infrastructure approach had only moderate impact to turret space, arm cabling mass and volume, and flight system resources. As an almost direct re-flight of MAHLI hardware, it enables all of the capabilities of the MSL MAHLI (Edgett et al. 2012) to be performed as well. WATSON and the higher resolution ACI, together, enable the most versatile and capable set of rover arm-mounted imagers ever flown to the surface of Mars. 


\subsubsection{SHERLOC Organics/Mineral/Chemical Detection}

A major NASA goal is to identify whether there is life elsewhere in the universe (NASA 2020). Life on Earth requires energy, liquid water, nutrients, and organic carbon, but the exact prescription for the origin of life on Earth has been lost to billions of years of geologic processing, chemical weathering, and biological processes. Soon after it formed, Mars likely also had the ingredients necessary to support an origin of life and, as such, is a prime candidate for the search for life elsewhere in the solar system. Mars has preserved a rock record-including sedimentary rocks that carry signals of very ancient environments - that is older than the oldest rocks on Earth (cf. Harrison 2020). Early Mars had energy in the form of impact melting and magmatism and it hosted nearly a dozen different types of aqueous, potentially habitable, environments during its first billion years (e.g., Murchie et al. 2009; Grotzinger et al. 2014). Organic material and nutrients would also have been present either through in situ synthesis of outgassed volatiles, such as $\mathrm{N}_{2} / \mathrm{H}_{2} / \mathrm{CO} / \mathrm{CO}_{2}$ (Chang et al. 1983), or through infall of meteorites and interplanetary dust particles (IDPs) (Flynn 1996; Flynn and McKay 1990). The chosen landing site at Jezero crater includes potential records of past hydrothermal, lacustrine, weathering, and diagenetic environments (Ehlmann et al. 2008, 2011; Glotch et al. 2010; Wray and Ehlmann 2011; Michalski et al. 2013). If Mars had life, Jezero is an ideal location to search for Martian biosignatures. SHERLOC will contribute to this investigation by providing micron-to-millimeter scale microscopy in combination with mineral and organic detection and characterization, putting any potential Martian biosignatures in their proper geologic and textural context.

To assess the presence of potential biosignatures, the SHERLOC spectrometer is used to detect classes of organics, minerals, and chemicals. Figure 2 shows spectral examples of detectable organics using DUV Raman, and for aromatic species, the fluorescence spectra. These classes of organics are found in life as we know it but a number of them have also been found in meteorites. We also show examples of organics that may be present as a result of predicted degradation paths resulting in oxidized organics. It should be noted that the DNA and RNA spectral features represent the capability to detect organic complexity versus subunits or condensed carbon; while not a definitive biomarker, detection of complex organics would certainly be classified as a potential biosignature. For mineral detection Fig. 3 shows examples of some mineral classes that are observable with SHERLOC. The majority of these would be indicative of an aqueous past and are key source and sinks for elemental cycling necessary for life. In addition, we have demonstrated that SHERLOC can detect silicate minerals such as the olivines and pyroxenes in the SaU 008 martian meteorite (Table 1, Fig. 43). It should be noted that increasing iron or manganese content in a sample will attenuate the spectral response through UV absorption of signal. Mineral specificity for carbonates and sulfates has also been demonstrated and an in-depth review can be found in Razzell Hollis et al. (2020b).

The next sections provide an overview of the Jezero crater site and its potential for organic preservation, background regarding the spectroscopy SHERLOC performs and how it detects organics and minerals, a discussion of the key subsystems and components and how they were tested and integrated to form SHERLOC, a demonstration of operations and performance, the methodology to operate, command, and calibrate SHERLOC on the surface of Mars, the SHERLOC data processing plan, and the efforts that were put into maintaining a level of required instrument cleanliness. Table 1 is a summary of the SHERLOC instrument, its component characteristics and performance values. 


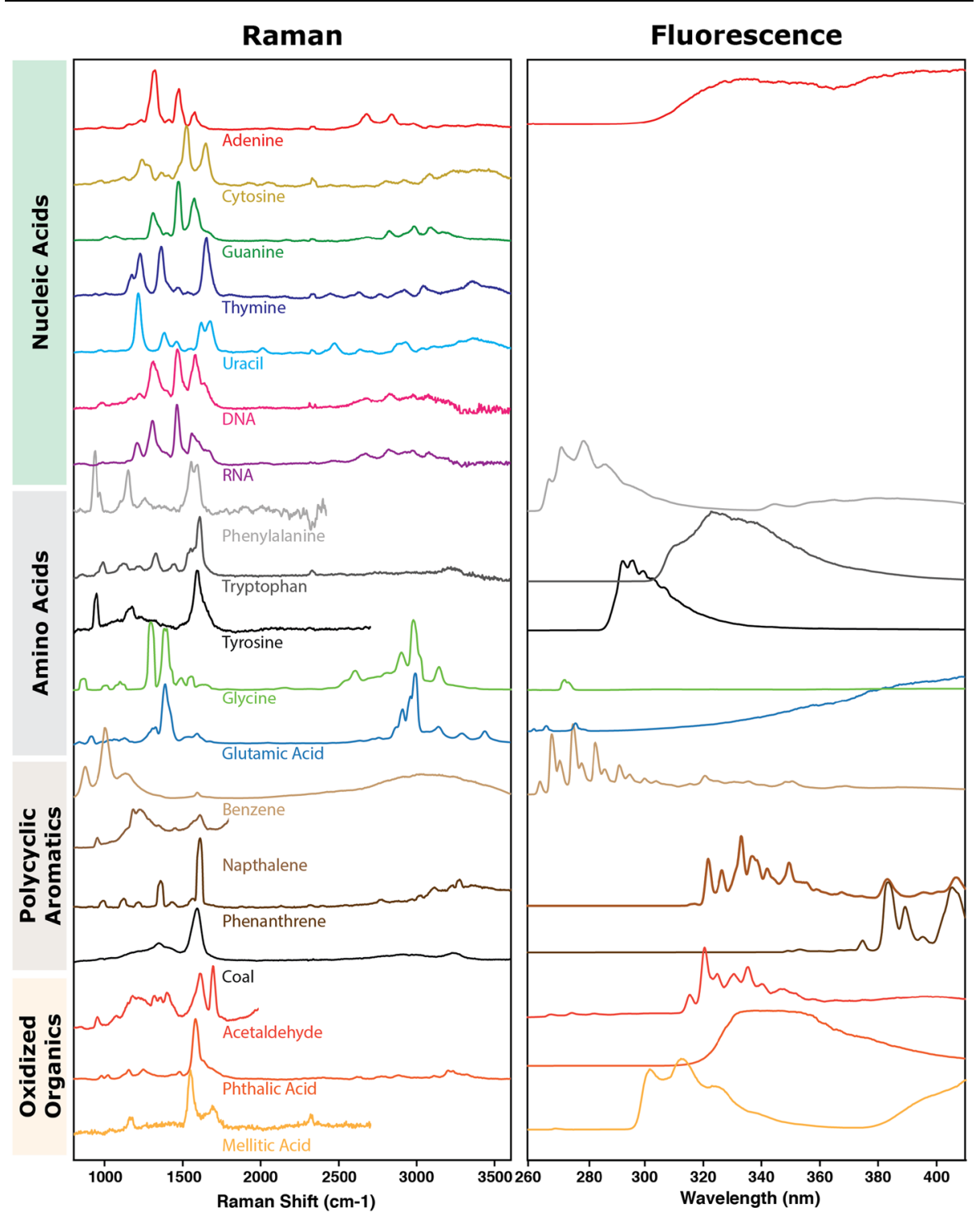

Fig. 2 Example spectra of organics detectable using SHERLOC DUV Raman (Left) and native fluorescence (right). These spectra were collected on the lab breadboard instrument that was used to set the SHERLOC performance requirements and are representative of what can be achieved using SHERLOC on Mars (The lab breadboard, MOBIUS, is described in detail in Bhartia et al. 2014; Abbey et al. 2017; Sapers et al. 2019; Razzell Hollis et al. 2020b)

\subsection{Jezero Crater Field Site}

After an exhaustive site selection process (Grant et al. 2018), Jezero crater was chosen to be the destination of the Perseverance rover (Stack et al. 2020) Jezero crater $\left(18.4^{\circ} \mathrm{N}, 77.7^{\circ}\right.$ E) is an $\sim 45 \mathrm{~km}$ diameter impact structure in the Nili Fossae region of Mars. Orbital data provide spectral and geomorphic evidence for a complex geological evolution that includes 


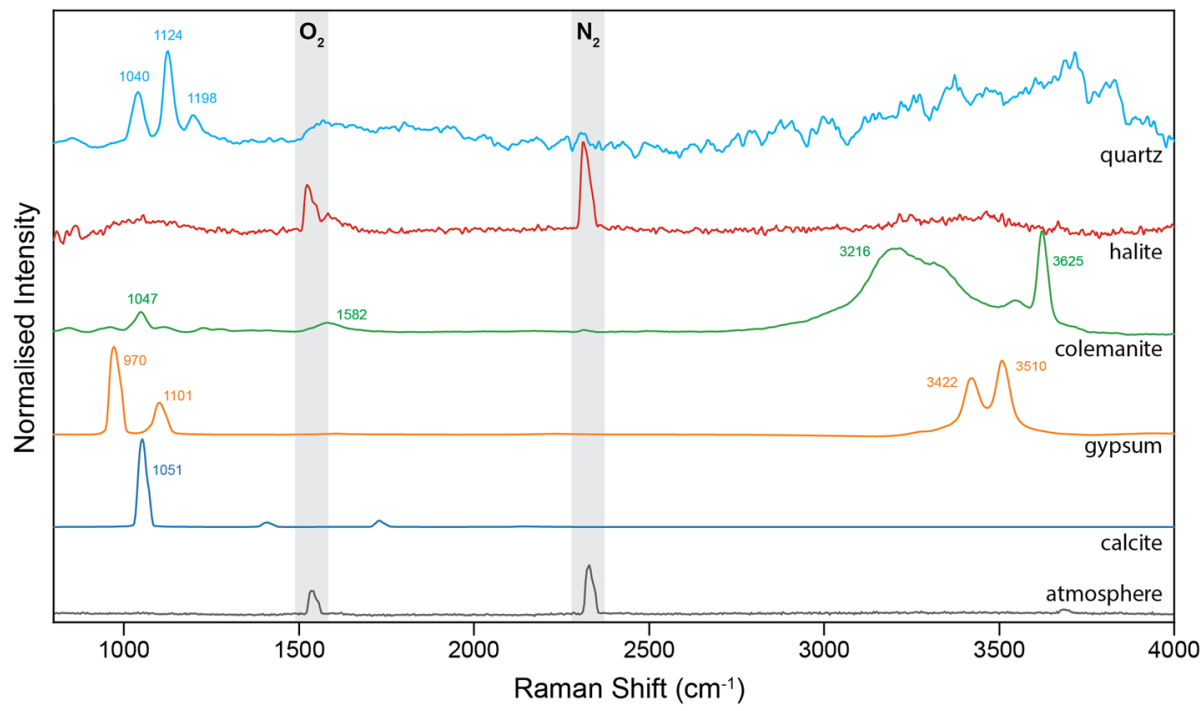

Fig. 3 Example spectra of minerals detectable using SHERLOC. These spectra were collected on the lab breadboard and are representative of what can be achieved using SHERLOC (Razzell Hollis et al. 2020b). The $\mathrm{O}_{2}$ and $\mathrm{N}_{2}$ lines are terrestrial atmospheric interferences that will not be observed on Mars

a history of fluid-rock interaction both inside and outside of the crater. The pre-Jezero basement rocks include primitive igneous crust marked by spectral signatures of low-Ca pyroxene (Mustard et al. 2008; Ehlmann et al. 2008), polymict megabreccias from the $\sim 3.96 \mathrm{Ga}$ Isidis impact (Werner 2008), and $\mathrm{Fe}$ - and $\mathrm{Mg}$-smectite-bearing rocks which suggest pronounced alteration by regional groundwater flow (cf. Mangold et al. 2007). Two valleys formed by fluvial incision enter Jezero crater from the west and north, and one exits the crater to the east (Fassett and Head 2005; Schon et al. 2012; Goudge et al. 2012). The complex history of Jezero crater includes the events that made it possible for such valleys to breach the crater rim (e.g., some combination of superposition and deep erosion of the crater rim). Jezero today is partly filled and rocks exposed at the surface include spectrally diverse olivine- and carbonate-bearing rocks, a high-Ca pyroxene-bearing unit, and fluvial-deltaic deposits bearing very little detectable pyroxene (Ehlmann et al. 2008; Horgan et al. 2020). The deltaic sediments and inlet and outlet valleys are key indicators that Jezero records the site of an ancient paleolake. Some investigators have proposed that the crater fill materials also include lava (Schon et al. 2012; Goudge et al. 2015). This complex history makes Jezero crater a relevant target for questions of Martian habitability, and for its potential to return quality petrologic and astrobiological samples.

Lithologic materials of the Jezero crater floor, for instance, consist of two primary phases: a light-toned unit that carries spectral signatures of olivine and carbonate, and a dark-toned, fracture-bearing unit that has spectral signatures consistent with high-Ca pyroxene (Horgan et al. 2020). Light-toned crater fill deposits have been interpreted as fine-grained detrital sediment deposited in a paleolake (Schon et al. 2012), as aeolian deposition within a potentially dry crater (Horgan et al. 2020), or as exposures of a regional mantling unit, perhaps tephra, that occurs throughout the Isidis-Nili Fossae region (Goudge et al. 2015; Kremer et al. 2019). Dark-toned crater floor deposits have been interpreted as having resulted from mafic lava flows (Schon et al. 2012; Goudge et al. 2015), pyroclastic air fall (Golder et al. 2020), or detrital (aeolian or fluvial) sedimentary deposition. A better understanding of the 
Table 1 SHERLOC summary

General Instrument Characteristics

\begin{tabular}{|c|c|c|}
\hline Hardware Units & Mass (kg) & Envelope (mm) \\
\hline SHERLOC Body Assembly (SBA) & 2.90 & $220 \times 89 \times 200$ \\
\hline SHERLOC Turret Assembly (STA) & 6.83 & $352 \times 176 \times 276$ \\
\hline SHERLOC Calibration Target (SCT) & 0.44 & $150 \times 33 \times 89$ \\
\hline Power by Mode & Power@28 V (W) & Peak Current (A) \\
\hline Imaging (with LEDs) & $\begin{array}{l}14.3 \text { (peak) } \\
12.2 \text { (avg) }\end{array}$ & 0.44 \\
\hline Spectroscopy (Detailed) & $\begin{array}{l}128.8 \text { (peak) } \\
60.5(\text { avg })\end{array}$ & 4.75 \\
\hline STA Warm up & 80.4 & 3.84 \\
\hline STA Survival & 19.1 & 0.91 \\
\hline STA Decon & 34.6 & 1.65 \\
\hline SCT Decon & 2.0 & 0.10 \\
\hline Resources & Number & Description \\
\hline Power Switch & 6 & $\begin{array}{l}\text { DEA - 2A } \\
\text { PDU - 10A } \\
\text { Warm up heaters - 4A } \\
\text { Survival heaters - 2A } \\
\text { STA Decon heaters - 2A } \\
\text { SCT Decon heaters - 2A }\end{array}$ \\
\hline Communication Interfaces & 2 & $\begin{array}{l}\text { DEA - LVDS } \\
\text { PDU - LVDS }\end{array}$ \\
\hline FS Monitored PRTs & 13 & $\begin{array}{l}2 \text { in SBA } \\
11 \text { in STA }\end{array}$ \\
\hline
\end{tabular}

SHERLOC Science and Operability Summary

System Performance

Fine scale classification for aromatic, aliphatic, and condensed $\quad<10^{-3}$ wt/wt over 100 um sampling carbon $\left(<100 \mu \mathrm{m}^{2}\right)$

Bulk scale detection for Aromatic organics $\left(<100 \mu \mathrm{m}^{2}\right)$

$10^{-5} \mathrm{wt} / \mathrm{wt}$ over $7 \times 7 \mathrm{~mm}$ area

Grain detection for minerals

50 micron grain size

\begin{tabular}{ll}
\hline Operability & \\
\hline Operational Time & $10-120$ minutes \\
Working distance & $48 \mathrm{~mm}$ from surface (nominal) \\
& $<30 \mathrm{~mm}$ for drill hole science (nominal) \\
Focus range of travel & $15.8 \mathrm{~mm}$ (spectroscopy) \\
& $16.7 \mathrm{~mm}$ (imaging) \\
Mapping Area (capability) & $7.8 \times 10.4 \mathrm{~mm}$ at target \\
Mapping Step Size & $>5$ microns \\
Spatial Resolution & Features as small as 50 microns \\
Surface Roughness tolerance & $+/-550$ microns \\
Arm placement accuracy tolerance & $\mathrm{XY}-11 \mathrm{~mm}$ \\
& $\mathrm{Z}-7 \mathrm{~mm}$ \\
Arm Jitter tolerance & $<10 \mathrm{microns}$ RMS \\
Arm drift tolerance & $<100 \mathrm{microns} / \mathrm{min}$ \\
\hline
\end{tabular}


Table 1 (Continued)

\begin{tabular}{|c|c|}
\hline Spectroscopy Specifications & Description \\
\hline \multicolumn{2}{|l|}{ Optical Properties } \\
\hline Slit Dimensions & $\begin{array}{l}100 \text { microns in dispersion direction } \\
150 \text { microns in the spatial direction }\end{array}$ \\
\hline $\mathrm{F} / \#$ & 7 \\
\hline FOV & $100 \times 150$ microns at the target \\
\hline Grating & $4200 \mathrm{~g} / \mathrm{mm}$ \\
\hline Target to slit Magnification & $1: 1$ \\
\hline \multicolumn{2}{|l|}{ Deep UV Raman } \\
\hline Spectral Range & $254-274 \mathrm{~nm}$ \\
\hline Spectral Resolution & $0.269 \mathrm{~nm}$ \\
\hline Spectral Throughput & $36 \%$ \\
\hline \multicolumn{2}{|l|}{ Fluorescence } \\
\hline Spectral Range & $275-354 \mathrm{~nm}$ \\
\hline Spectral Resolution & $0.269 \mathrm{~nm}$ \\
\hline Spectral Throughput & $24 \%$ \\
\hline \multicolumn{2}{|l|}{ Detector } \\
\hline Product & e2v 42-10 CCD \\
\hline Array size & $2048 \times 512$ pixels \\
\hline Pixel Scale & 13.5 microns/pixel \\
\hline $\begin{array}{l}\text { Read Noise, Conversion gain, } \\
\text { Saturation (Complete single chain) }\end{array}$ & $\begin{array}{l}\text { High gain: } 5.8 \text { e- rms, } 0.46 \mathrm{e}-/ \mathrm{DN}, 28 \mathrm{ke}-(\mathrm{ADC} \text { limited }) \\
\text { Low gain: } \sim 10 \mathrm{e}-\mathrm{rms}, 2.35 \mathrm{e}-/ \mathrm{DN}, 101 \mathrm{ke}-(\mathrm{CCD} \\
\text { limited) }\end{array}$ \\
\hline Dark Noise@273 K & 80 e-/pixel \\
\hline $\begin{array}{l}\text { Phase Change Material Transition } \\
\text { Temperature (SCCD temp) }\end{array}$ & $-28 \mathrm{C}$ \\
\hline \multicolumn{2}{|l|}{ Deep UV Laser } \\
\hline Laser Type & 248.5794 nm Deep UV Hollow Cathode Laser \\
\hline Minimum Spot Size at target & 111 microns $\left(1 / \mathrm{e}^{2}\right)$ \\
\hline $\begin{array}{l}\text { Spot size at spectrometer focus @ } \\
\text { room temp/pressure }\end{array}$ & $\begin{array}{l}120.1 \text { microns in the dispersion direction } \\
123.2 \text { microns in the spatial direction }\end{array}$ \\
\hline Path throughput & $63 \%$ \\
\hline Energy (Beginning of Life) & $>14 \mathrm{uJ}$ \\
\hline Life Capability & $7 \mathrm{x}$ mission life \\
\hline Pulse Width & 20-40 usec (adjustable) \\
\hline Frequency & 10-160 hz (adjustable) \\
\hline \multicolumn{2}{|l|}{ Scanner } \\
\hline Scanner Type & 2-axis $(\mathrm{Az} / \mathrm{El})$ voice coil fast steering mirror \\
\hline Range of motion & $\begin{array}{l}+/-61 \text { mrad (SHERLOC only uses }+/-28 \text { mrad of motion } \\
\text { to achieve full } 7 \times 7 \text { mapping capability) }\end{array}$ \\
\hline Scale Factor & $\begin{array}{l}11.5 \mathrm{mRad} / \mathrm{V}(\mathrm{Az}) \\
10.9 \mathrm{mRad} / \mathrm{V}(\mathrm{El})\end{array}$ \\
\hline Position Error & $<0.18 \mathrm{mrad}$ (22 microns at the target) \\
\hline
\end{tabular}


Table 1 (Continued)

Spectroscopy Specifications Description

Spectral Mapping Functions

Modes

Spectra

Single spectra taken based on user defined commanded position

Point

$\mathrm{N}$ point spectral maps based on user defined table and commanded central position. Maps are located in NVM or can be loaded into VM prior to observation

Area

$\mathrm{N}$ area spectral maps based on user defined table and commanded central position. Maps are located in NVM or can be loaded into VM prior to observation. Area maps are collected by moving the scanner over specified area during SCCD acquisition

Max Map Size 1296 points, limited by raw data buffers. Shape of tables can be arbitrary as they are a table of defined az/el positions for scanner motion

Max Pulses per Point

2000 pulses per point, limited by photodiode sampling buffers

Max Number of Spectral Regions

3; SCCD can be vertically binned with selectable gain setting per region

Spectra Types

$1 \mathrm{D}$ and $2 \mathrm{D}$ (single spectra only)

Onboard Advanced Software Functions

Data Preprocessing

Background subtraction, laser normalization, bad pixel removal and/or cosmic ray removal (functions are parameterizable and selectable)

Spatial Binning

Spatially reduce spectral maps to using binning tables loaded on instrument

Spectral Binning

Spectrally reduce data sets to up to 6 spectral bins

Region of Interest Selection

Rank and selection regions of interest using weighted average of up to 6 spectral bins over entire map area

Data Buffers

1 raw and 4 process buffers

Imaging

ACI-specific Specifications

\begin{tabular}{ll}
\hline Nominal Working Distance & $48 \mathrm{~mm}$ \\
Focus Range & $\pm 8.35 \mathrm{~mm}$ from working distance \\
Focusing accuracy & $34 \mu \mathrm{m}$ \\
MTF & 0.75 at best focus and 0.3 at $+/-250$ microns at 20 cycles \\
& per $\mathrm{mm}(50$ microns at target) averaged over FOV \\
Depth of Field & $+/-250 \mu \mathrm{m}$ \\
Field of View & $16.16 \mathrm{x} 12.12 \mathrm{~mm}$ with $11.7 \mathrm{~mm} \emptyset$ unvignetted; \\
Focal Ratio (f/number) & 3.8 \\
Band Pass & $500-600 \mathrm{~nm}$ (grayscale) \\
Pixel Scale & $10.1 \mathrm{microns} /$ pixel \\
\hline WATSON-specific Specifications & \\
\hline Working Distance Range & $17.8 \mathrm{~mm}$ to Infinity \\
Depth of Field (see also Ghaemi & $\sim 0.045 \mathrm{~cm}$ at 22.5 cm working distance \\
2009, Fig. 15) & $\sim 1 \mathrm{~cm}$ at $20 \mathrm{~cm}$ working distance \\
& $\sim 30 \mathrm{~cm}$ at 1 m working distance \\
\hline
\end{tabular}


Table 1 (Continued)

\section{Imaging}

MTF

Field of View

Focal Ratio (f/number)

Band Pass

Pixel Scale

(comparable to MER MI/MSL MAHLI)
0.173 at system Nyquist freq. (min working distance)

0.221 at system Nyquist freq. (infinity focus)

$41^{\circ}$ diagonal (min. working distance)

$39^{\circ}$ diagonal (infinity focus)

9.80 (min. working distance)

$\sim 8.47$ (infinity focus)

394-670 nm (color)

$13.1 \mu \mathrm{m} /$ pixel (min. working distance)

$32.0 \pm 0.7 \mu \mathrm{m} /$ pixel $(69 \mathrm{~mm}$ working distance)

$100 \pm 8 \mu \mathrm{m} /$ pixel (255 mm working distance)

Detector (ACI and WATSON)

Products

Array size

Pixel size

Cover glass

Microlenses

Bayer pattern color microfilters

system gain, read noise, full well
ACI - OnSemi KAI-2020M interline transfer CCD

WATSON - OnSemi KAI-2020CM interline transfer CCD 1640 x 1214 pixels; $1600 \times 1200$ photoactive pixels

$7.4 \mu \mathrm{m}$ (square pixels)

No

ACI - yes

WATSON - yes

ACI - no

WATSON - yes

WATSON - 15.84 e-/DN, 18.7 e-, 24507 e-

Illumination Source (ACI and WATSON)

Sun

White light LEDs

Ultraviolet LEDs
Nominal operations use daytime lighting conditions in direct sun or shadowed

four LEDs arranged in two groups of two; each group can be operated independently or together; Avago Technologies HSMW-10x LEDs

two LEDs, operated together; $365 \mathrm{~nm}$ emission; Nichia NSHU550B

Exposure (ACI and WATSON)

\begin{tabular}{ll}
\hline $\begin{array}{l}\text { Duration } \\
\text { Auto-exposure }\end{array}$ & $\begin{array}{l}0 \text { to } 838.8 \text { seconds in } 0.1 \text { milliseconds increments } \\
\text { Developed by Maki et al. (2003) }\end{array}$ \\
\hline video acquisition (ACI and WATSON) & $<2 \mathrm{~Hz}$ \\
\hline Frame rate & \\
\hline Compression (ACI and WATSON) & No compression \\
\hline uncompressed & $\sim 1.7: 1$ compression \\
Lossless & JPEG with user defined quality between 1 and 100 \\
Lossy & $\begin{array}{l}\text { Uncompressed images remain onboard SHERLOC DEA; } \\
\text { compression specified upon later transmission using } \\
\text { Deferred compression }\end{array}$ \\
& rover Virtual Data Product (VDP) function \\
\hline
\end{tabular}


Table 1 (Continued)

Imaging

Focus merge (ACI and WATSON)

\begin{tabular}{ll}
\hline Products & Best focus image and range map \\
Max number of images & 8 images, no larger than $1600 \times 1200$ pixels \\
Input image quality & Raw, uncompressed \\
Output compression & JPEG with user defined compression quality \\
Processing Options & merge, registration (feature tracking + corner detection), \\
& blending (spline-based)
\end{tabular}

Calibration Targets

External (SCT)

\begin{tabular}{ll}
\hline 1. AlGaN on Sapphire $(262 \mathrm{~nm})$ & Spectral Calibration standard \\
2. Diffusil & Diffuse Ambient Light (Decontamination Heated) \\
3. Mars Meteorite & Sayh al Uhaymir 008 (SaU 008) collected in Oman in \\
& 1999 (Decontamination Heated) \\
4. Maze & Intensity/Spatial standard (Decontamination Heated) \\
5. AlGaN on Sapphire $(335 \mathrm{~nm})$ & Spectral Calibrations standard \\
6. Front: Polycarbonate Spacesuit & HOEMD/EPO/Resolution target
\end{tabular}

Visor Material

Back: Geocache Coin

7. Vectran HOEMD soft goods

8. Orthofabric HOEMD

9. Teflon HOEMD

10. nGimat coated Teflon HOEMD

Internal (Located inside the SHERLOC ACI dust cover)

AlGaN on Sapphire $(274 \mathrm{~nm}) \quad$ Spectral/Intensity standard

origins of these crater floor units is critical to reconstructing the age and time-line of deposition within Jezero crater, defining times of active deposition and erosion, and exploring the extent to which the Jezero system may have recorded perennial versus ephemeral lacustrine sedimentary environments. Potentially mineralized fractures that cross-cut crater floor materials (Miklusicak et al. 2020) may also provide a glimpse into later, diagenetic fluid flow in the subsurface of Jezero crater.

A second critical region of the Jezero landing site includes distinct deposits along the crater's western rim that have been interpreted as resulting from fluvial-deltaic deposition (e.g., Fassett and Head 2005; Goudge et al. 2015; Goudge et al. 2017). Deltaic deposition served as a critical element in the selection of Jezero crater as the Mars 2020 landing site, as it records strong evidence that the crater, at least at one time, hosted a water-rich lacustrine environment that would have received chemical nutrients (as well as potential organic materials) from a broader region of the Martian surface. Strata in the deltaic deposits are of particular interest as both potential sinks for organic deposition (e.g., fluvial overbank deposits, prodelta muds, etc.) and to provide critical input to reconstruct the depositional history of Jezero crater (e.g., periods of increased or decreased fluvial input to a lake). Such reconstructions will produce distinct hypotheses for identifying circumstances of the spa- 
tial and temporal extent of habitability and the identification of lithologies with the greatest astrobiological potential for sample return.

A third critical region of Jezero crater consists of a spatially discrete carbonate-bearing unit that is located along the western margin of the crater, near the edge of the western delta. This unit exhibits the strongest spectral signatures for Mg-carbonate in the Jezero system and has been interpreted as either resulting from weathering of an ultramafic tephra deposit, widespread in the Isidis region (Kremer et al. 2019), or as lacustrine carbonates deposited in ancient shoreline environments of a former lake (Horgan et al. 2020). This so-called marginal carbonate unit is particularly intriguing as an astrobiological target. Groundwater alteration of mafic to ultramafic igneous rocks, for instance, would provide an aqueous environment of circum-neutral $\mathrm{pH}$ that might be conducive to microbial habitability. Similarly, lacustrine shoreline deposits may provide a locus for authigenic carbonate formation, such as in spring deposits and shoreline groundwater seeps that may be highly conducive to both microbial growth and organic preservation.

\subsection{Biosignature/Organic Preservation Potential at Jezero}

The current Martian surface is an inhospitable place for most organic molecules due to high UV flux and an oxidizing component present within the ubiquitous dust that is present on the surface (Hecht et al. 2009; Moores and Schuerger 2012; ten Kate et al. 2006). However, organic material has been identified in Gale crater by the Sample Analysis at Mars (SAM) investigation (Mahaffy et al. 2012) on MSL (e.g., Glavin et al. 2013; Freissinet et al. 2015; Stern et al. 2015; Eigenbrode et al. 2018), as well as in Martian meteorites (Steele et al. 2016). SAM observed 150-300 ppb of chlorobenzene, and chlorinated alkanes at lower abundances (Glavin et al. 2013; Freissinet et al. 2015), as well as thiophene and methythiophene in samples of lacustrine mudstones (Eigenbrode et al. 2018). In addition, the Martian meteorites (e.g., Allan Hills (ALH84001)) are well-studied samples from Mars in which hydrocarbon compounds, including polycyclic aromatic hydrocarbons (PAHs), have been identified in more than a dozen different meteorites (Grady et al. 1997, 2004; Grady 2006; Steele et al. 2006, 2012a,b). Steele et al. (2016) tabulated all of the bulk analyses from shergottites to calculate that igneous rocks on Mars could possess between $\sim 18 \pm 26 \mathrm{ppm}$ reduced carbon. Given this evidence, it is highly likely that organic molecules are preserved just beneath the Martian surface in Jezero crater (Carrier et al. 2019).

\section{The SHERLOC Investigation Objectives}

\subsection{Science Objectives}

The SHERLOC investigation, along with other payload elements, such as Planetary Instrument for X-ray Lithochemistry (PIXL), SuperCam, Mastcam-Z, RIMFAX, and Mars Environmental Dynamics Analyzer (MEDA) (Allwood et al. 2020; Wiens et al. 2021; Maurice et al. 2021; Bell et al. 2020; Hamran et al. 2020; Rodriguez-Manfredi et al. 2021) will provide critical data to decipher the evolutionary history of Jezero crater, reconstruct the environmental history of the Jezero lacustrine systems, and identify samples most likely to preserve potential biosignatures. The observations that acquire these data map directly to the SHERLOC investigation objectives (Table 2).

SHERLOC will be used to assess the habitability potential and aqueous history at Jezero crater through identification of key minerals using Raman spectroscopy. Observations of 
Table 2 SHERLOC investigation objectives

In situ Astrobiology

Assess the habitability potential and aqueous history of a sample

Assess the availability of key elements and energy sources for life (C, H, N, O, P, S, etc.)

Determine if there are potential biosignatures preserved in Martian rocks and outcrops
DUV Raman/ACI Imaging

DUV Raman

DUV Raman/Fluorescence/Imaging

In-situ Mineralogy/Geology

Contribute to grain-scale characterization of rock type, facies, stratigraphy and modern regolith and aeolian sediment

Obtain fine-scale (10-100 $\mu \mathrm{m} /$ pixel) images to document the location, context, and grain-scale details of SHERLOC

Spectrometer and PIXL proximity science targets with an option to also image SuperCam targets in the robotic arm workspace

Provide core sample extraction support, including data needed for tool placement (e.g., range-finding) and curatorial images of core extraction sites, core hole walls, and cuttings

WATSON/ACI Imaging

WATSON/ACI Imaging

WATSON Imaging

Sample Return Support

Provide organic and mineral analysis for selective sample caching

Engineering Support

Provide engineering support for rover operations including diagnostic imaging, wheel and instrument inspections, etc.

Deep UV Raman/Florescence/ACI Imaging WATSON Imaging

clay minerals, carbonates, sulfates, and halides in sedimentary units has shown that Mars hosted nearly a dozen different types of aqueous (alkaline, acidic, and saline lakes, hydrothermal deposits etc.) and other potentially habitable environments during its first billion years (Ehlmann et al. 2008; Glotch et al. 2010; Wray and Ehlmann 2011; Michalski and Niles 2012; Elhmann et al. 2011; Murchie et al. 2009). Some minerals are particularly indicative of environmental conditions such as serpentine (alkaline, reducing), sulfate (acidic, oxidizing), and carbonates (alkaline). Other phases are useful as environmental indicators, although only when they are found in mineral assemblages in which multiple phases collectively allow derivation of fluid geochemistry. By collecting point mineralogical data over an extended spatial scale in conjunction with textural evidence, it is possible to discern whether aqueous minerals formed in sediments, hydrothermal precipitates, volcanic materials, or resulted from secondary overprinting by weathering or diagenesis. Measurements on these scales can identify many important features including evidence for aqueous activity, alteration features, sedimentary laminae, or melt pockets, and can contribute to the analysis of detrital grains in sediments or individual clasts in conglomerates or breccias. These detailed textural observations will be made possible by SHERLOC's ability to image from the micron to the multi-millimeter scale; this range is an important size-step in the continuum of contextual investigations that make up the 2020 mission.

Furthermore, by identifying minerals with Raman spectroscopy, SHERLOC will also be able to assess the availability of key elements and energy sources for life. Life on Earth is 

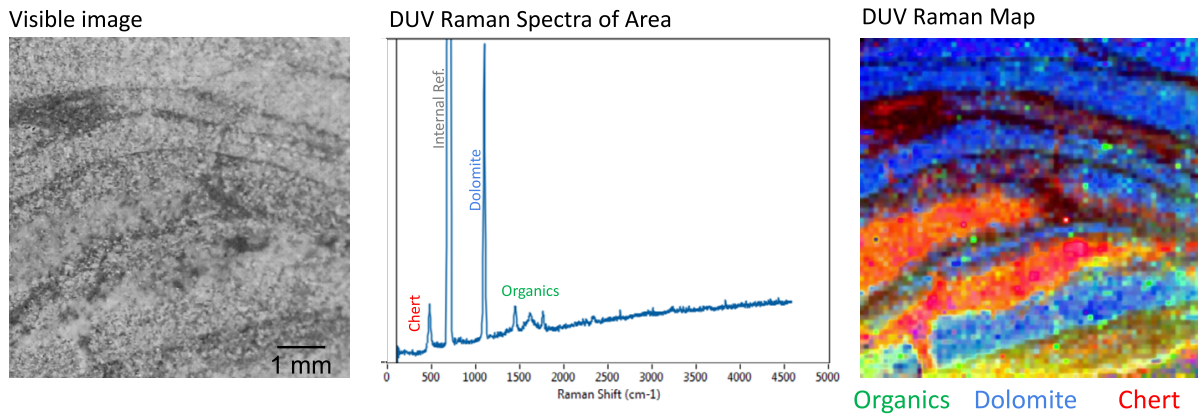

Fig. 4 Example of SHERLOC science via analysis of a Strelley Pool Formation Stromatolite sample using the laboratory breadboard MOBIUS instrument (see Bhartia et al. 2014; Abbey et al. 2017). The chert signature observed is highly attenuated by the edge filter but is observable in this sample. Colors blend in the map and organics are green and yellow as they are mixed with the chert signature (sample courtesy of A. Allwood)

driven by oxidation-reduction reactions that form and transform organic matter and mineral substrates containing key elements such as $\mathrm{C}, \mathrm{H}, \mathrm{N}, \mathrm{O}, \mathrm{P}$ and $\mathrm{S}$. The abundance and diversity of these substrates in a sedimentary environment are key measures of habitability and analysis of these substrates in a given environment can identify sedimentary rocks that reflect the detrital component, a precipitated residue of the dissolved component, or post-depositional (diagenetic) alteration processes (Fries et al. 2011). Transformation of minerals by biology or diagenesis has distinctive signatures that can be observed in assemblages of astrobiologically important minerals (e.g., carbonates, nitrates, phosphates, sulfates, etc.). The presence of such minerals, especially in association with organics, or when they otherwise imply some degree of chemical or morphologic disequilibrium, is an important component in evaluating potential biogenicity.

SHERLOC data will also be used to ascertain whether there are potential organic biosignatures preserved in Martian rocks and outcrops by combining Raman spectroscopy with fluorescence spectroscopy (Bhartia et al. 2012b, 2014; Abbey et al. 2017). Potential biosignatures that can be identified and spatially resolved using these synergistic techniques are key organics such as hopanes, steranes, and organic macromolecules. This especially strong interrogative power will help identify samples to be obtained for return to Earth. SHERLOC's operational modes can determine the organics and minerals present over scales that match the mineralogy and morphology of most biosignatures resulting from microorganisms (Bhartia et al. 2010). It is capable of correlating detected classes of organics with morphology (widths and shapes) to determine whether morphological candidates for microfossils, filaments, or stromatolitic layering are potentially biogenic (Allwood et al. 2006) (Fig. 4). For finer-scale features, SHERLOC's micro-mapping mode is a platform for nondestructive, sub-picogram sensitive organic detection (Bhartia et al. 2010), providing an in situ capability unprecedented for a flight instrument.

SHERLOC will further provide organic and mineral analyses that will be essential for selective core sample extraction and caching. By generating maps of organic molecules and mineral features co-located with corresponding contextual information in the form of micron-to-millimeter scale imaging, the team will be able to identify the most compelling samples that need to be acquired for sample return. In addition, its autofocus and intrinsic depth of field enables organic/mineral analysis of the near-subsurface by observing the inside walls of the extracted cores (i.e. boreholes) where organic material can be protected from the oxidizing nature of the surface. 
Table 3 lists the SHERLOC investigation goals associated with the Spectroscopy element of the investigation. All of the SHERLOC scientific objectives and observations described above include options for operations at any time of the Martian day. The inherent Martian UV flux, coupled to the limited spectroscopic field of view, with internal instrument baffling, and the speed that the spectrometer Charged Coupled Device (SCCD) for spectroscopy is read, enables day-time spectroscopic observations. The only notable exception, based on MSL MAHLI experience, might be some hardware inspections (e.g., rover deck) and rare science objectives (e.g., borehole walls), that might be best imaged at night using onboard illuminators (Minitti et al. 2014; Yingst et al. 2016).

\subsection{Geologic Photography at 10-100 $\mu \mathrm{m}$ Scales}

SHERLOC's two robotic arm deployable cameras, WATSON and ACI, will provide high spatial resolution $(10.1 \mu \mathrm{m} /$ pixel via ACI; 13.1 to $>100 \mu \mathrm{m} /$ pixel via WATSON), color (WATSON) and grayscale (ACI) images of rocks and regolith in natural and roboticallydisturbed (e.g., abraded, cored, broken by wheels) states. WATSON will, furthermore, obtain images of landscapes, rocks hidden from view of other Perseverance cameras (e.g., beneath ledges, etc.), and provide the contextual spatial resolution bridge between SHERLOC and PIXL observations and those of the rover's mast-mounted instruments (e.g., Yingst et al. 2016).

Close-up photography of geological materials, including fossil biosignatures, is a field almost as old as photography itself (e.g., Deane 1861; Woodward 1903; Kerp and Bomfleur 2011). Photography is used in the geosciences for a wide range of purposes designed to illustrate, instruct, document, and provide new data for study-including examination of changes that occur on various time scales and images used to construct three-dimensional models of geological materials (e.g., Stow 2005; Maley 2005; Remondino et al. 2010; Rodrigues 2015; Fonstad and Zettler-Mann 2020).

High spatial resolution imaging (in this case, 10.1 to $\sim 100 \mu \mathrm{m} /$ pixel) of geological materials on Mars began with the $31 \mu \mathrm{m} /$ pixel grayscale robotic arm deployable Microscopic Imager (MI) cameras onboard the MER rovers, Spirit and Opportunity (Herkenhoff et al. 2003, 2019). The Phoenix Mars Scout lander included a Robotic Arm Camera (RAC; Keller et al. 2008) as well, which provided sand grain-scale views $(\sim 50 \mu \mathrm{m} /$ pixel $)$ of regolith particulates (Goetz et al. 2010). MSL's Chemistry Camera (ChemCam) Remote Microscopic Imager (RMI) provides sub-millimeter per pixel grayscale close-up views of rocks and regolith from a mast-mounted instrument (Le Mouélic et al. 2015). In addition, the robotic arm-mounted MAHLI onboard Curiosity (Edgett et al. 2012) routinely obtains images in the 16 to $100 \mu \mathrm{m} /$ pixel range; it is capable of $13.9 \mu \mathrm{m} /$ pixel but rarely is it practical for the camera to be positioned close enough to a target to obtain images at that scale (Yingst et al. 2016). Together, Minitti et al. (2013) and Yingst et al. (2016) described the many versatile uses of the MAHLI; Edgett and Newsom (2018) noted examples of changes detected (sand grain motion) during single MAHLI imaging sessions; and Garvin et al. (2017) and Caravaca et al. (2020) described methods for three dimensional Structure from Motion (SfM) model construction from MAHLI data. All of the capabilities and uses of the MSL MAHLI are achievable with the SHERLOC WATSON camera.

All ACI images will be obtained at $\sim 10.1 \mu \mathrm{m} /$ pixel in gray scale. ACI will enable three primary tasks: (1) determine the best focus position for the spectrometer, (2) correlate the laser position with the target surface to provide morphological context of SHERLOC spectra and chemical maps, and (3) place the high resolution chemical maps in context with the rest of the target. In addition, the ground-based data processing pipeline also uses the imaging 
Table 3 SHERLOC Spectroscopic Investigation Matrix. Mars 2020 Mission Goals and derived Mission Science Objectives (Farley et al. 2020) are mapped into SHERLOC contributions and data products addressed by the SHERLOC investigation

\begin{tabular}{|c|c|c|c|c|}
\hline $\begin{array}{l}\text { Mars } \\
2020 \\
\text { Obj }\end{array}$ & $\begin{array}{l}\text { Derived Mission Science } \\
\text { Objectives }\end{array}$ & SHERLOC Analysis & SHERLOC Data Product ** & Notes \\
\hline \multirow[t]{2}{*}{$\leftarrow$} & $\begin{array}{l}\text { aqueous conditions existed } \\
\text { and identify organic mineral } \\
\text { assemblages that constrain } \\
\text { chemical and redox } \\
\text { environment) }\end{array}$ & $\begin{array}{l}\qquad \text { Classify mineral } \\
\text { assemblages associated with } \\
\text { aqueous activity. }\end{array}$ & $\begin{array}{l}\text { DUV Raman spectra } \\
\text { of carbonates, sulfates, } \\
\text { phyllosilicates, zeolites, etc. } \\
\text { with grain sizes }>50 \mu \mathrm{m} \text {. }\end{array}$ & \multirow{2}{*}{$\begin{array}{l}\quad \text { DUV Raman } \\
\text { signatures of } \\
\text { astrobiologically important } \\
\text { organic and mineralogical } \\
\text { species describe energy } \\
\text { sources, ancient } \\
\text { environmental conditions } \\
\text { and water availability at a } \\
\text { time when the surface of } \\
\text { Mars was warmer and } \\
\text { wetter. }\end{array}$} \\
\hline & $\begin{array}{l}\text { Assess availability and } \\
\text { distribution of electron donors, } \\
\text { key elements and energy } \\
\text { sources }\end{array}$ & $\begin{array}{l}\text { Detect and classify } \\
\text { CHNOPS elements in } \\
\text { minerals and organics. } \\
\text { Identify alterations in } \\
\text { mineral content due to } \\
\text { biologic processes. }\end{array}$ & $\begin{array}{l}\text { DUV Raman spectra } \\
\text { of CHNOPS minerals in } \\
\text { organics/minerals over an } \\
\text { area at } 50 \mu \mathrm{m} \text { resolution. }\end{array}$ & \\
\hline \multirow{3}{*}{ 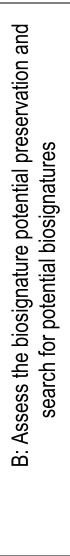 } & $\begin{array}{l}\text { Characterize } \\
\text { physiochemical processes and } \\
\text { conditions in the } \\
\text { paleoenvironment, and identify } \\
\text { mechanisms for formation and } \\
\text { preservation of biosignatures }\end{array}$ & $\begin{array}{l}\quad \text { Classify organics } \\
\text { within mineralogical context } \\
\text { and assess the probability of } \\
\text { origin by meteoritic infall, } \\
\text { native, and/or biologically } \\
\text { derived processes. }\end{array}$ & $\begin{array}{l}\quad \text { Textural, } \\
\text { fluorescence maps of } \\
\text { organics \& DUV Raman } \\
\text { maps of organics and } \\
\text { minerals. Includes grain size } \\
\text { analysis }(>50 \mu \mathrm{m}) \text { and } \\
\text { distribution. }\end{array}$ & \multirow{3}{*}{$\begin{array}{l}\text { Combining } \\
\text { fluorescence and DUV } \\
\text { Raman signatures for bulk } \\
\text { and fine scale organic and } \\
\text { mineral maps of } \\
\text { surface/near-surface } \\
\text { material describes the } \\
\text { potential for organic } \\
\text { preservation and potential } \\
\text { biosignatures detection. } \\
\text { Combining mineral and } \\
\text { organic maps enables in } \\
\text { situ assessment of } \\
\text { formation and alteration } \\
\text { processes for a given target } \\
\text { to assess whether materials } \\
\text { are meteoritic, native and/or } \\
\text { past life and chemical } \\
\text { precursors. Also constrains } \\
\text { the potential for } \\
\text { biosignature preservation. }\end{array}$} \\
\hline & $\begin{array}{l}\text { Chara } \\
\text { type, and timir } \\
\text { processes tha }\end{array}$ & $\begin{array}{l}\text { oxidation, } \\
\text { via organic }\end{array}$ & $\begin{array}{l}\text { DUV Ram } \\
\text { organics and mine }\end{array}$ & \\
\hline & $\begin{array}{l}\quad \text { Map distributions of } \\
\text { potential biosignatures (PBS) } \\
\text { relative to geologic features, } \\
\text { including characteristics of } \\
\text { potentially biogenic structures }\end{array}$ & $\begin{array}{l}\quad \text { Assess organic } \\
\text { classes (aromaticity, } \\
\text { functional groups, PBS, etc.) } \\
\text { and correlating aqueous- } \\
\text { related mineralogy and } \\
\text { textures on surfaces and drill } \\
\text { holes. }\end{array}$ & \multirow{2}{*}{$\begin{array}{l}\quad \text { DUV Raman and } \\
\text { fluorescence maps of } \\
\text { organics and minerals co- } \\
\text { located with textures and } \\
\text { morphology of the target } \\
\text { surface over a } 5 \times 5 \mathrm{~mm} \text { area } \\
\text { with spatial resolution of } \\
100 \mu \mathrm{m} \text {. drill hole mapping } \\
\text { of mineral and organic } \\
\text { species to } 25 \mathrm{~mm} \text { depth. }\end{array}$} & \\
\hline \multirow{2}{*}{ 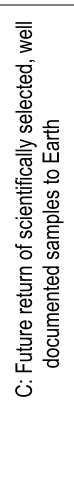 } & $\begin{array}{l}\text { Assess evidence for } \\
\text { past life, or its chemical } \\
\text { precursors, and place } \\
\text { constraints on the past } \\
\text { habitability and the potential for } \\
\text { preservation of the signs of life }\end{array}$ & \begin{tabular}{l}
\multicolumn{1}{c}{ Assessment of } \\
mineral/ textural \\
characteristics consistent \\
with PBS, energy sources, or \\
preservation. Identification of \\
preserved evidence \\
suggesting biotic or pre-biotic
\end{tabular} & & \multirow{2}{*}{$\begin{array}{l}\text { Facilitates merger } \\
\text { of organic/mineral data with } \\
\text { other payload instruments } \\
\text { to improve analytical } \\
\text { capability of the rover } \\
\text { instrument suite. } \\
\text { Identification of samples } \\
\text { with organic composition is } \\
\text { vital to selection of samples } \\
\text { for return. The direct } \\
\text { analysis of drill holes } \\
\text { results in a proxy for core } \\
\text { analysis. SHERLOC's } \\
\text { mapping mode inside the } \\
\text { drill hole can identify } \\
\text { organic gradients that } \\
\text { indicate degradation of } \\
\text { organic material. }\end{array}$} \\
\hline & $\begin{array}{l}\text { Assess the history and } \\
\text { significance of surface } \\
\text { modification processes } \\
\text { including but not limited to: } \\
\text { impact, photochemical, } \\
\text { volcanic, and aeolian. } \\
\text { Constrain the magnitude, } \\
\text { nature, timing and origin of } \\
\text { past planet wide climate } \\
\text { change ( }\end{array}$ & $\begin{array}{l}\text { Assess morphologic } \\
\text { and mineralogical } \\
\text { characteristics of Mars' } \\
\text { paleoenvironment with co- } \\
\text { located organic molecule } \\
\text { mapping, mineral phase } \\
\text { mapping, and contextual } \\
\text { images. Identify salt, } \\
\text { aqueous products, and } \\
\text { evaporite concentrations. }\end{array}$ & $\begin{array}{l}\text { Deep UV Raman and } \\
\text { fluorescence maps of organic } \\
\text { molecules co-located with } \\
\text { perchlorates or other } \\
\text { oxidizers and textures, (grain } \\
\text { size, shape, distribution) co- } \\
\text { located with surface features. }\end{array}$ & \\
\hline
\end{tabular}

data to correct for arm drift during prolonged measurements. The resulting imaging system also provides additional science capabilities such as range-finding with $\sim 0.2 \mathrm{~mm}$ accuracy from a safe standoff from the surface, $10.1 \mu \mathrm{m} /$ pixel views of rock and regolith texture and structure, ability to generate of 3D surface relief maps via MAHLI heritage Z-stacks. The ACI was built with the MAHLI heritage UV Light Emitting Diodes (LEDs) which will enable high-resolution imaging of mineral fluorescence, if present, in a sample (Fig. 5). 

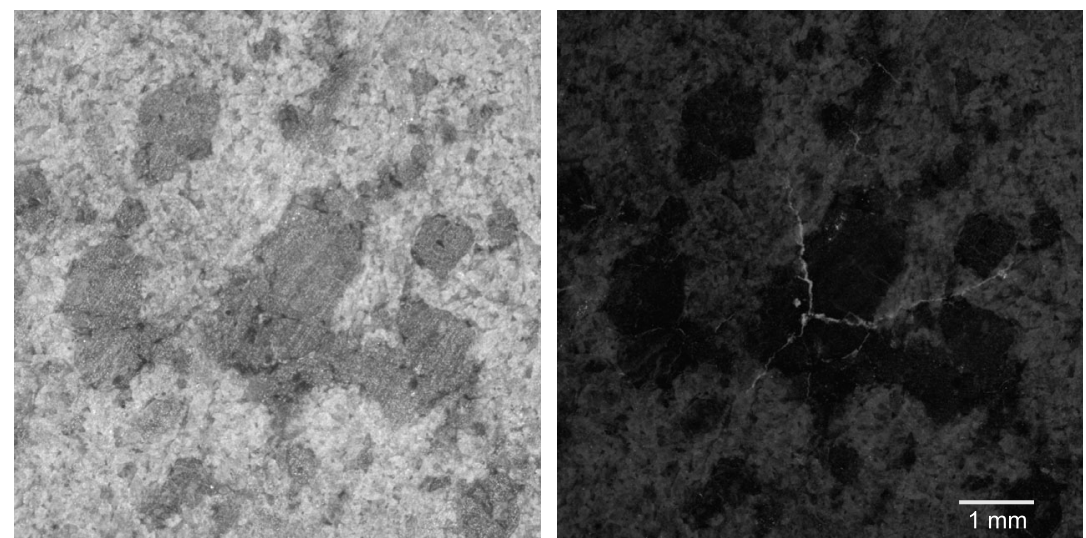

Fig. 5 ACI visible LEDs and UV LED illuminated images of the SAU 008 meteorite on the flight spare SHERLOC Calibration Target during SHERLOC thermal-vacuum test. ACI image with visible LEDs (Left). Same region with UV LEDs showing the florescence from carbonates from terrestrial contamination

a)

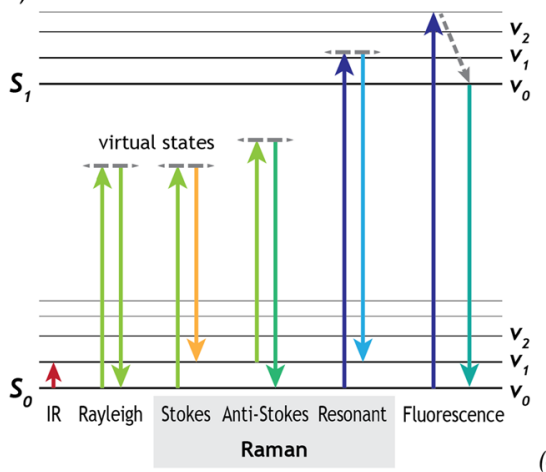

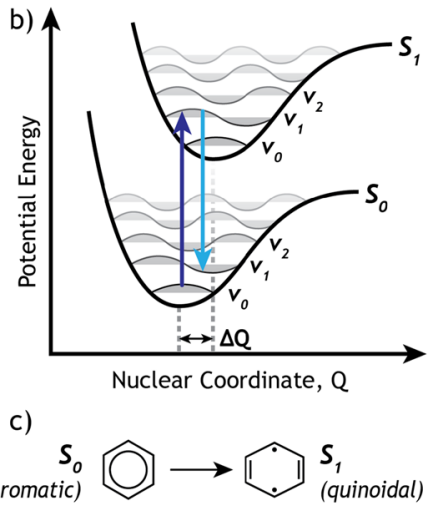

geometry change exaggerated

Fig. 6 (a) Jablonski diagram illustrating electronic (S) and vibrational (v) energy levels of a molecule, and the transitions involved in each scattering process (Raman) or emission (fluorescence). (b) the Morse potentials and vibrational wavefunctions for ground and excited electronic states involved in resonant Raman scattering. (c) the change in nuclear geometry for electronic excitation of unsubstituted benzene

\subsection{Deep UV Laser Induced Native Fluorescence Spectroscopy}

Native fluorescence was first observed in Acuña et al. (2009) and since then, the variety of compounds that exhibit native fluorescence has been determined to be extensive. Most fluorescent compounds incorporate aromatic ring structures but there are some exceptions (Borkman and Kearns 1966). In simple terms, fluorescence provides insight on the electronic states of a molecule. It requires that a molecule absorbs an incident photon such that a bound electron can jump from an $\mathrm{S}_{0}$ to an $\mathrm{S}_{1}$ or $\mathrm{S}_{2}$ state. Once excited, vibrational, rotational, and molecular collisions cause the excited electron to non-radiatively lose energy until, typically, it reaches the lowest vibrational level of the $S_{1}$ excited energy state. The gap between the lowest energy level of $S_{1}$ and various levels of the $S_{0}$ ground state will dictate the emission wavelength produced when the electron returns to $S_{0}$ (Fig. 6a). This excita- 
tion energy is lost through thermal energy or by emission of a photon that is less energetic than the initial absorbed photon; all of this can occur within in hundreds of picoseconds of the incident excitation. The wavelength of this emitted photon provides information about the gap between electronic levels. Typically, as the physical size of a compound increases the emitted photon is of a lower energy (longer wavelength); the shift from the excitation wavelength to the emission wavelength is known as the Stokes shift.

Native fluorescence is a complimentary mode of detection to Raman, and resonance Raman. One primary benefit are cross-sections that are $10^{5}$ to $10^{8}$ times larger than Raman. Additionally, when excitation is below $250 \mathrm{~nm}$, native fluorescence enables observation of the full range of fluorescence emissions from the full range of aromatic organics including benzene, the simplest aromatic organic - which has an emission at $260 \mathrm{~nm}$ (Asher and Johnson 1984) - to polyaromatic hydrocarbons (PAHs) such as anthracene, which can absorb a $248 \mathrm{~nm}$ photon and emit at $374 \mathrm{~nm}$ (Bhartia et al. 2008). As a result, the full spectral shape of simple and complex aromatic organics is characterizable and provide a very sensitive method for determining molecular size. Indeed, DUV native fluorescence can be used to detect and differentiate astrobiologically relevant organics (aromatic amino acids, PAHs, and biosignatures) from mineral backgrounds (Bhartia et al. 2008). It was subsequently demonstrated that DUV native fluorescence enables detection of microbes at the single cell level on natural basalt samples, demonstrating spatially resolved detection of picograms of carbon (Bhartia 2012; Bhartia et al. 2010, 2012a). Salas et al. (2015) demonstrated that the high radiance of a laser is necessary to observe these weak emissions and employed the technique in instruments for in situ measurements that observe trace organic and microbial detection in the ocean subsurface on the walls of igneous boreholes. More recently, laser-induced DUV native fluorescence was demonstrated to detect and locate and spatially resolve the stochastic nature of microbes and organics, including PAHs, in situ in Greenland ice (Malaska et al. 2020).

The sensitivity of laser-induced DUV native fluorescence enables detection of exceedingly trace amounts of organics. While classification is possible, and further enabled with spatially resolved maps, the specificity is limited by the broad emission peaks and spectral variances that are typically observed and interpretable. However, as described by (Bhartia et al. 2014) and others (Bhartia et al. 2012b; Hug et al. 2018), native fluorescence can be used to detect regions of interest, followed by DUV resonance Raman to further interpret an unknown substance. Given that even resonance Raman is inherently orders of magnitude weaker, it is not an ideal broad area search tool for in situ analysis due to resource (i.e. power, data volume, laser lifetime etc.) constraints, however the specificity of the spectrum provides a higher level of organic differentiation. Fusion of these data can be used to extract additional information (Bhartia et al. 2012b). Thus, combining these two complementary methods leverages both techniques to enable detection and characterization of organics and the mineral matrix.

Typically, only organic molecules fluoresce when excited at $248.6 \mathrm{~nm}$, especially between the 260 to $370 \mathrm{~nm}$ region where SHERLOC observations occur. However, as there is evidence of some mineral fluorescence from some astrophysical environments (Garstang 1957; Bowen 1960; Hartman and Johansson 2000; Johansson et al. 2000), SHERLOC data analysis will include study of the elemental data obtained by PIXL on the same target (Allwood et al. 2020) to assess the probability of mineral interference. Additionally, the deep UV Raman analysis will provide confirmation of fluorescence features as organics. 


\subsection{Deep UV Raman Spectroscopy}

Raman spectroscopy is a form of molecular vibrational spectroscopy that is highly sensitive to the chemical structure, environment, and photochemical properties of molecules in a target or sample. In contrast to infrared (IR) spectroscopy, which involves the direct excitation of molecular vibrations by absorption of IR radiation, Raman spectroscopy probes these vibrations using the Raman effect, a phenomenon where incident light is inelastically scattered by molecular vibrations, and the change in photon energy corresponds to the change in vibrational energy. Any given molecule may have several Raman-active vibrational modes of different energies, depending on chemical structure, which leads to a typical spectrum of several Raman peaks between 10 and $4000 \mathrm{~cm}^{-1}$. Because of its sensitivity to vibrations of the $\mathrm{C}-\mathrm{C}$ and $\mathrm{C}=\mathrm{C}$ bonds (in the $1000-1800 \mathrm{~cm}^{-1}$ range) that make up the backbone of organic molecules, the Raman spectrum in this range is sometimes referred to as the "molecular fingerprint" region. Laboratory Raman spectroscopy can be used to study isotope effects, molecular orientation/anisotropy, crystallinity, and surface adsorption (Asher and Murtaugh 1988; Toyama et al. 1999; Chennaoui Aoudjehane et al. 2012; Le Ru and Etchegoin 2008; Razzell Hollis 2016; Fries et al. 2011; Fries and Steele 2018).

In the next few paragraphs, we briefly explain the principles behind Raman spectroscopy, but we recommend Raman Spectroscopy by Long (1977) for a more detailed and authoritative description. A molecule absorbs an incident photon and its energy is briefly raised to a virtual state between the electronic ground state $\left(S_{0}\right)$ and electronic excited state $\left(S_{1}\right)$, which exists for $\sim 1$ fs before relaxing back to the ground state through re-emission of the scattered photon (illustrated in Fig. 6). Typically, the molecule returns to its original vibrational state, meaning that the scattered photon has the same energy as the incident photon (elastic scattering); this process is Rayleigh scattering. Roughly 1 in $10^{7}$ scattering events result in the molecule relaxing back to a different vibrational state, such that the scattered photon loses energy (inelastic scattering). The wavelength shift is characteristic of a vibrational mode in the molecule. Finally, the more coherent the excitation source is, the finer the scattered photons are and the more readily a molecular identification can be made which is why lasers are commonly used for Raman spectroscopy.

The selection rule for Raman scattering dictates only those vibrational modes that involve a change in the molecule's polarizability, $\alpha$, at equilibrium, are Raman active and contribute to the Raman spectrum. Compare this to the selection rule for IR absorption, which requires that vibrational modes involve a change in dipole moment to be IR active. Consequently, Raman spectra tend to be dominated by the vibrational modes of easily polarized bonds like $\mathrm{C}-\mathrm{C}$ and $\mathrm{C}=\mathrm{C}$, which usually lack inherent dipole moments and thus are weak in IR. Because of this, Raman and IR spectroscopy are considered complementary techniques that probe different aspects of a sample's molecular vibrations. Traditional Raman scattering cross-sections tend to be very small compared to fluorescence and Rayleigh scattering typically $10^{-30} \mathrm{~cm}^{2} \mathrm{sr}^{-1}$ per molecule (Asher and Murtaugh 1988) - necessitating the use of high-power lasers as an excitation source.

One of the most important considerations in Raman spectroscopy is the choice of excitation wavelength. The difference in intensity between Raman scattering and other photophysical phenomena; fluorescence, for example, can be $\sim 10^{10}$ times stronger than Raman, meaning that the Raman spectrum may be impossible to detect if the frequency of the scattered light coincides with a strong fluorescence peak (Asher and Johnson 1984; Tarcea et al. 2007). To emphasize the importance of wavelength, an excitation just above the DUV, at 262 $\mathrm{nm}$, can lead to obscuration by fluorescence (Christensen et al. 2008). Additionally, scattered intensity is generally proportional to the fourth power of the scattered frequency, which for 
Raman depends on the excitation frequency, $f_{L}$, and the frequency of the vibration, $f_{v}$. This means that, for the same laser power, a typical $\mathrm{C}=\mathrm{C}$ Raman peak at $1600 \mathrm{~cm}^{-1}$ will generate $\sim 25 \mathrm{x}$ more signal under $248.6 \mathrm{~nm}$ excitation as compared to the more common 532 $\mathrm{nm}$ excitation. However, including the resonance term (Asher 1993), the observed increase can be as high 100 to 200x greater (Nagli et al. 2008). While the majority of commercial Raman instruments operate in the visible to near-IR range $\left(\lambda_{\mathrm{ex}}=400-1000 \mathrm{~nm}\right)$, the development of commercial deep UV lasers $\left(\lambda_{\text {ex }}<250 \mathrm{~nm}\right)$ has triggered greater interest in UV Raman spectroscopy in recent decades, especially as UV Raman spectra occur in a relatively fluorescence-free spectral range (Asher 1993; Storrie-Lombardi et al. 2001; Tarcea et al. 2007; Hug et al. 2009; Tuschel et al. 2010; Bhartia et al. 2012b; Bhartia et al. 2014; Beegle et al. 2015). In addition to the increase in signal from high-frequency excitation, the use of an excitation frequency that coincides with the absorption band of a molecule can increase scattering cross-sections a further 10-1000x. This phenomenon, known as molecular resonance, is exploited in resonant Raman spectroscopy to increase signal and achieve a greater spectral sensitivity to chemical and environmental perturbations of molecular structure (Asher and Johnson 1984). Pre-resonance refers to the tendency for scattering crosssections to increase faster than the fourth power rule as excitation wavelength approaches the electronic transition energy (Johnson and Asher 1987).

The origin of resonant Raman is the virtual state coinciding with a real vibrational energy level of the electronically excited state. This leads to strong electron-phonon coupling between vibrational excitation and electronic excitation, described by the Franck-Condon overlap between original state (e.g. $\left.S_{0}, v_{0}\right)$ and excited state $\left(S_{1}, v_{1}\right)$, shown in Fig. 6b. Strong overlap between $v_{0}$ and $v_{1}$ only occurs when the electronic transition involves a net change in nuclear geometry $Q$ (the equilibrium positions of atoms in the molecule), as illustrated in Fig. 6c, and only applies to vibrational transitions that explore a similar change in nuclear geometry. This tends to result in the selective enhancement of those vibrational modes associated with the part of the scatterer that absorbs light (known as the chromophore), which for organic molecules under visible or UV excitation is usually a conjugated $\pi$-system such as an aromatic ring. Because of the sensitivity of resonance to changes in nuclear geometry, resonant Raman spectroscopy is a powerful tool for studying how chemical and environmental factors perturb the structure of the chromophore and/or its excited state. Vibrational overtones, transitions between $v_{0}$ and $v_{2}$, are also frequently observed under resonant conditions as a second, weaker, set of Raman peaks at slightly less than twice the Raman shift of the fundamental transition (Long 1977). While resonance provides many advantages for detection and identification of chromophores at low concentration, quantitative analysis may be complicated by the absorption of incident and/or scattered light by the chromophore (Pelletier 2003; Wu et al. 2006; Razzell Hollis et al. 2020b).

A number of laboratory studies have been conducted using SHERLOC analog instruments to demonstrate the application of UV Raman spectroscopy to planetary science and astrobiology. The use of DUV excitation $(248.6 \mathrm{~nm})$ provides resonance with many organic molecules that possess at least one aromatic ring, a list that includes several molecules of biological significance (such as the nucleobases of RNA and DNA, and certain amino acids), as well as many compounds that are formed by abiotic processes and have been found in meteorites (such as polyaromatic hydrocarbons and carbonaceous material) (Kubasek et al. 1985; Wu et al. 2001; Sephton et al. 2002; Frosch et al. 2007; Czaja et al. 2009; Höhl et al. 2016). Raman scattering cross-sections for these molecules under UV are of the order of $10^{-25} \mathrm{~cm}^{2} \mathrm{sr}^{-1}$, meaning that a detectable Raman spectrum can be obtained with a relatively low-power laser, even when the aromatic organic material is at low concentration (Asher and Murtaugh 1988; Abbey et al. 2017). Saturated aliphatic compounds, which lack 
the conjugated $\pi$-system that acts as a chromophore, are still detectable. However, with only pre-resonance or without resonance enhancement, they typically have a higher limit of detection than aromatics (Trulson and Mathies 1986; Bhartia et al. 2014; Beegle et al. 2015; Abbey et al. 2017). The resonant enhancement of certain molecules and vibrational modes under UV can be very useful when dealing with complex samples such as biological material, leading to cleaner spectra dominated by a more manageable number of key molecules that are easier to classify (Asher and Johnson 1984; Nelson et al. 1992; Hug et al. 2009; Sapers et al. 2019). We have also demonstrated the ability to conduct quantitative analysis of concentration from Raman spectral intensities, provided that self-absorption and the shape of the annular laser spot are considered (Razzell Hollis et al. 2020b). Mineralogy can also be mapped at the sub-mm scale, as many Mars-relevant minerals (carbonates, borates, sulfates, some silicates) may be distinguished by the positions of key peaks in the 800-1200 $\mathrm{cm}^{-1}$ range (Abbey et al. 2017; Razzell Hollis et al. 2020a). SHERLOC also avoids the complication of spectra dominated by background fluorescence, as the $248.6 \mathrm{~nm}$ excitation ensures that Raman peaks between $\sim 800$ and $4000 \mathrm{~cm}^{-1}$ occur in a narrow spectral range $(252-275 \mathrm{~nm}$ ) that is spectrally separated from fluorescence that occurs at $>275 \mathrm{~nm}$ (Asher and Johnson 1984; Tarcea et al. 2007; Bhartia et al. 2008, 2014; Hug et al. 2009; Beegle et al. 2015). Analysis of complex organic systems is possible with UV Raman, where deconvolution of the Raman spectra can be used to identify multiple organic species, including cells themselves when present in a sample (Sapers et al. 2019). We have developed a model that accounts for these physical parameters (Bhartia et al. 2014). This model was vital in driving the SHERLOC optical design requirements and operation methodology and was used to evaluate successful performance of the completed instrument.

\subsection{SHERLOC Sensitivity Model}

The advantage of DUV fluorescence spectroscopy is its high sensitivity in detecting the presence of aromatic organic compounds when compared to other forms of spectroscopy, including mass spectroscopy. In environments such as Mars, where organics are rare, the low detection limit can result in identification of rocks/surfaces that should be cored and cached for sample return as well as a way to document the concentration of organics to identify potential contamination sources when these samples are analyzed in terrestrial laboratories. However, while fluorescence can detect, the advantage of DUV resonance Raman is its chemical specificity such that organic classification and mineral identification are enabled.

In the development of the instrument concept, a photon budget model (Bhartia et al. 2010; Hug et al. 2012; Bhartia et al. 2014; Abbey et al. 2017; Razzell Hollis et al. 2020b) was used to define the performance of the SHERLOC instrument in order to detect aliphatic organics at $<1 \times 10^{-3} \mathrm{wt} / \mathrm{wt}$ in a $100 \mu \mathrm{m}$ spot; the most challenging case of mission objectives. Initially the model included known terms such as laser power, Raman cross sections and ambient light levels as well as estimates for instrument performance such as optical throughput. This enabled requirements to be placed on individual subsystems to use as requirements in their design. As the instrument went through design, development and characterization, the model was modified to include the measured performance of key subsystems, including the laser energy at the sample, the optical throughput (illumination and detection), detector quantum efficiency, dark noise and end to end electronics read noise in order to ensure that the instrument requirements were all satisfied with margins.

In brief, the sensitivity of the measurement is a function of the interaction volume that is collectable on the CCD, the fluorescence or Raman cross section, the concentration of organics and the number of UV photons. The interaction volume can be modeled as a cylinder 
with diameter of the laser beam and a height defined by the lesser of the value of the depth of field or the penetration of UV light into and out of a mineral matrix. Measurements of the depth of penetration into mars relevant samples, indications that the depth of field of the optical system ( $\sim 500$ microns) is on order of the depth of penetration (Carrier et al. 2019). Based on the measured SHERLOC beam parameters, the focused laser spot has an effective area at $\sim 7540 \mu \mathrm{m}^{2}$ (using the $1 / e^{2}$ definition), which encompasses $\sim 95 \%$ of total intensity. For a typical laser pulse energy of $8 \mu \mathrm{J}$ at start of mission in Jezero crater, we predict the dosage received by the sample will be $0.1 \mathrm{~J} / \mathrm{cm}^{2}$ per pulse. For a 5-pulse survey acquisition, the total dosage is $0.5 \mathrm{~J} / \mathrm{cm}^{2}$, and for a 400-pulse detailed acquisition, the dosage is $40 \mathrm{~J} / \mathrm{cm}^{2}$.

Using these values, we modeled the 3D distribution of laser intensity at the sample and determined that for a non-attenuating, non-subsurface-scattering sample, SHERLOC has an interrogation volume of $\sim 20 \mathrm{~nL}\left(\sim 0.02 \mathrm{~mm}^{3}\right)$. Given the detection limit of $\sim 10$ parts per million and assuming a sample density similar to that of basalt, we will be able to detect the Raman scattering from as little as $0.5 \mathrm{ng}$ of condensed carbon, or approximately 4 picomoles of a small organic compound such as adenine. For samples that are rich in organics and/or minerals that have strong absorption in the UV, attenuation of both incident laser light and Raman-scattered light will reduce the interrogation volume and increase the minimum concentration required for detectable Raman signal.

We have also considered that the DUV laser can lead to photolytic sample degradation (the low peak power avoids thermal alteration). Based on laboratory degradation studies and modeling of ambient UV in Jezero crater (Razzell Hollis et al. 2020c), the UV dosage received by the sample during operation of the SHERLOC laser is estimated to be comparable to 0.01-2 sols of exposure to solar UV radiation, depending on the number of laser pulses used per point. For longer exposures, e.g. detailed scan activities, the SHERLOC laser can induce photo-chemical degradation in organic materials (Razzell Hollis et al. 2020c). However, the extent of degradation and its impact on observed Raman and fluorescence spectra will depend on the organic in question, with more mature material (e.g. macromolecular carbons and carbonaceous material) expected to be highly resilient under UV exposure. Organic material may also be protected by the surrounding mineral matrix, as some certain minerals (such as clays) have been shown to inhibit UV-induced degradation of key biosignatures like DNA (Fornaro et al. 2018).

\section{Instrument Accommodation}

\subsection{Instrument Overview}

The SHERLOC instrument consists of three hardware units: (1) the SHERLOC Body Assembly (SBA) mounted within the rover chassis which houses the command and data handling, power distribution and scanner control units; (2) the SHERLOC Turret Assembly (STA) mounted on the robotic arm that houses the laser, scanner, spectrometer, imagers (ACI and WATSON) and local control electronics; and (3) the SHERLOC Calibration Target (SCT) mounted to the front of the rover chassis below the elbow bracket that includes spectral standards and HOEDM soft materials.

The SHERLOC instrument contains two electrically separated subsystems: Spectroscopy and Imaging. These subsystems have independent data and power connections to the rover and functions are interlaced through the commanding architecture. The Spectroscopy and ACI are optically co-boresighted for execution of spectral activities. Figure 7 shows the block diagram for SHERLOC. 


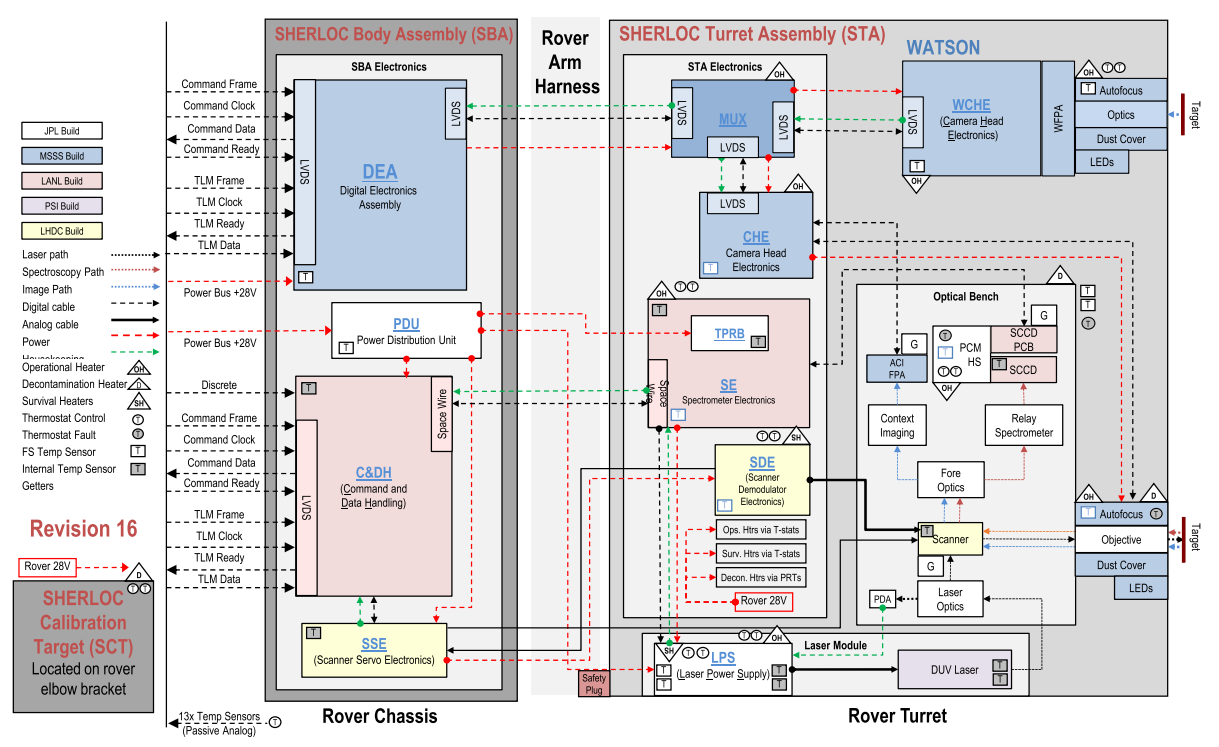

Fig. 7 SHERLOC instrument block diagram

\subsection{Hardware Locations}

The SBA is mounted within the rover chassis directly on the rover avionics mounting panel (RAMP). The thermally-controlled environment within the RAMP reduces the overall allowable flight temperature (AFT) range for electronics to $-40{ }^{\circ} \mathrm{C}$ to $+50{ }^{\circ} \mathrm{C}$. This enables the electronics to avoid the Mars seasonal and diurnal thermal swings without additional thermal control.

The STA is mounted on the turret at the end of the robotic arm with PIXL, the corer, the gas Dust Removal Tool (gDRT), and the facility contact sensor (Moeller et al. 2021). The robotic arm environment is much more severe than that inside internal the rover. The nonoperational AFT range from $-95^{\circ} \mathrm{C}$ to $+50{ }^{\circ} \mathrm{C}$; therefore the STA includes survival heaters and operational heaters to reduce the AFT range within the specification and capabilities of the hardware.

The SCT is mounted to the front of the rover chassis below the robotic arm elbow bracket. The SCT position allows the five degrees of freedom robotic arm to access the calibration targets with both the Spectrometer/ACI and WATSON subsystems.

\subsection{Harnessing}

The SBA and turret assembly harness, ADLIR (Acquisition \& Data Lines between Instrument and Rover), are connected through an impedance-controlled harness that is composed of $>12 \mathrm{~m}$ of round wire and flex cabling. The harness contains all intra-instrument data, power, and sensor signals. Mitigations in the harnessing and system design were included early in the SHERLOC design phase to avoid the type of issues observed on MSL for both MAHLI and the Alpha Particle X-Ray Spectrometer (APXS) regarding sensitivity of signal to external noise sources picked up through their harness (Gellert et al. 2015). For the spectroscopy subsystem, the development of the turret power regulation board (TPRB) use 
of temperature cycling resistant electronics (TRCE) chip-on-board $(\mathrm{CoB})$ technology was implemented to provide low-noise regulated power at the turret assembly for sensitive electronics. The analog scanner electronics are housed in the turret assembly to avoid noise feedback in the pointing control circuitry allowing for higher spatial resolution observations of SHERLOC targets. Additionally, the spectroscopy subsystem communication used a SpaceWire interface to ensure optimal data transfer limits greater than those observed on MSL. During SHERLOC development, the Los Alamos National Laboratory (LANL) demonstrated $>10$ megabits per second using flight hardware, allowing for no data storage being required in the spectroscopy electronics, leading to a simpler design. However, both the ACI and WATSON images are limited to 5 megabits per second per low-voltage differential signaling (LVDS) line, and the camera head readout rates are limited to 2.5 megapixels per second, as these electronics remain mostly unchanged from the MSL MAHLI design (Edgett et al. 2012).

\subsection{Vibration Isolation System}

Due to the vibrational environment on the turret caused by the adjacent percussive corer, the SHERLOC instrument is isolated from the turret feed structure by a hexapod canister-spring isolation system (Fig. 8). The design of the six struts uses preloaded springs exclusively operating under compression to attenuate natural frequencies thus reducing the energy transfer to the instrument. This results in a fully constrained, quasi-kinematic isolation system. Additionally, the isolation system includes flexure joint interfaces to ensure no stress is applied to the SHERLOC chassis during installation or drilling and coring operations to maintain optical alignments in all configurations.

\subsection{Ground Sensing Methods}

Unlike arm mounted instruments on MSL, instrument specific contact sensors were descoped from the Perseverance design and replaced with a facility contact sensor mounted to the rear of the turret feed structure (Moeller et al. 2021). The purpose of the facility contact sensor is to provide direct measurements relative to the rover coordinate system for accurate placement of the turret tools and instruments, including SHERLOC, along the axis of approach of turret instrumentation.

However, when that is not available, knowledge of the position of a proximity science (SHERLOC, PIXL) target in three-dimensional space can be determined from surface relief products derived from stereo pair imaging by Perseverance's engineering stereo cameras (Maki et al. 2020). Also, based on MSL MAHLI experience (Minitti et al. 2013; Robinson et al. 2013; Yingst et al. 2016), deployment of WATSON for range finding via autofocus imaging of a small portion of a geological target can also provide additional, reliable information. While the MSL MAHLI camera head was enveloped by a contact sensing device (Edgett et al. 2012), a similar capability is not included for WATSON. Except for some early testing, the MAHLI contact sensor was not engaged operationally nor accidentally during $>3100$ sols of Mars surface operations so the need for such a contact sensor has not been demonstrated and it was removed.

\subsection{Positioning}

SHERLOC is placed into operating position by the Perseverance's robotic arm. The accuracy of the placement is governed predominately by the thermal environment, accuracy 
Fig. 8 Image of the SHERLOC Turret Assembly on the rover's robotic arm with a view of the DUV laser and the vibration isolators

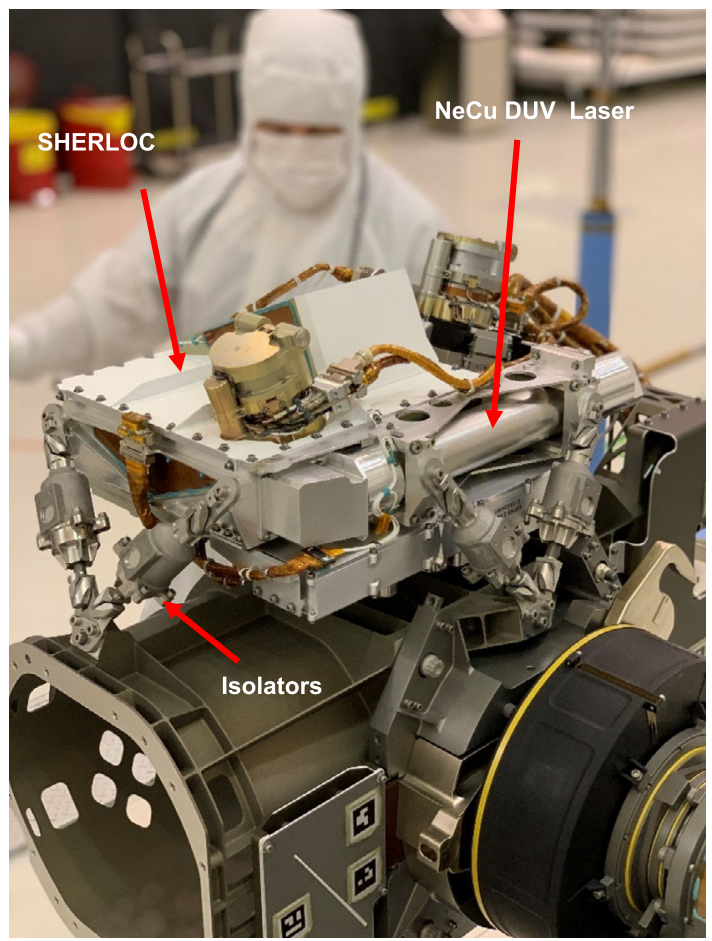

of the rover engineering camera models, and the mechanical accuracy of the robotic arm. SHERLOC operations will be also constrained for placement by the results of the facility contact sensor assessment of the target (relative placement) and for optimal placement, within a restricted thermal range of operations (nominally $10^{\circ} \mathrm{C}$ ). Nominally, this gives an $\mathrm{XY}$ placement accuracy (lateral location relative to the surface) of $< \pm 6 \mathrm{~mm}$ and $\mathrm{Z}$ placement accuracy (distance from the ground aligned to the optical axis) of $< \pm 5 \mathrm{~mm}$ and $<$ $\pm 2 \mathrm{~mm} X Y Z$ with thermal constraint. Relative unconstrained thermal operations are limited to placement accuracies of $\pm 11 \mathrm{~mm}$ for $\mathrm{XY}$ and $\pm 7 \mathrm{~mm}$ for $\mathrm{Z}$.

Operations can be performed in a single placement activity while meeting science requirements. The MSL robotic arm observed jitter of $<7$ microns root mean squared (RMS) providing a highly stable environment for spectral collection and imaging. For Perseverance, the robotic arm is bigger thus necessitating a new jitter measurement which will take place early in the mission. For extended spatial operations, SHERLOC will capitalize on the nudge capability of the robotic arm (steps of $\sim 3 \mathrm{~mm}$ ) to generate spectral and imaging mosaics.

\subsection{Data and Power Interfaces}

The SHERLOC power and data architecture leverages the functions of the Mars 2020 rover. Communication, command, and control use a command-reply handshake over LVDS with the Rover computing elements (RCEs) thus requiring the rover to be awake during all SHERLOC operations. Power is applied directly to the unit from the Rover Power Avionics Module (RPAM) which independently controls power to the SHERLOC spectroscopy and imaging subsystems and thermal zones for warm up, decontamination and survival heating. Ad- 
Fig. 9 The SHERLOC Body Assembly that houses the DEA and the $\mathrm{C} \& \mathrm{DH}$

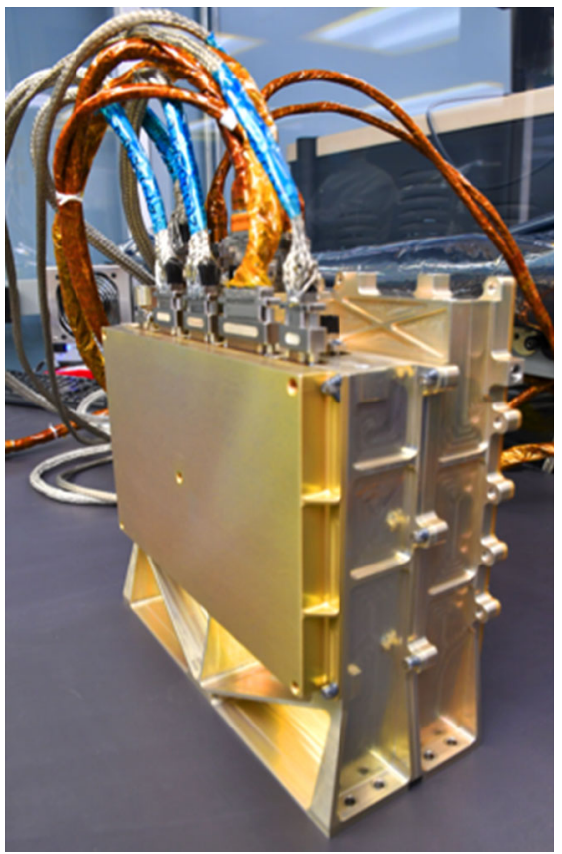

ditionally, SHERLOC is monitored by 13 flight system platinum resistance thermometers (PRTs) spread throughout the instrument to assess instrument temperature during flight.

\section{SHERLOC Body Assembly (SBA)}

The SBA houses the command and data handling and power electronics for the Spectroscopy and Imaging subsystems and serves as the interface between the SHERLOC turret assembly the rover's onboard computers (Fig. 9). To maintain the flight heritage of the SHERLOC imaging system, the SBA contains two distinct LVDS command and power interfaces to the Perseverance rover - one for imaging for the Digital Electronics Assembly (DEA), and one for the spectroscopy for the Command \& Data Handling (C\&DH)/Power Distribution Unit (PDU). The SBA's mass is $2.90 \mathrm{~kg}$ and it has an enveloped volume of $220 \mathrm{~mm}$ x $89 \mathrm{~mm} \mathrm{x}$ $200 \mathrm{~mm}$ excluding connectors and cabling.

\subsection{Power Distribution Unit (PDU)}

The PDU consists of six converters and dual electromagnetic interference and electromagnetic compatibility (EMI/EMC) filters to distribute power to the spectroscopy subsystem and accepts 22-36 V unregulated rover bus power. The PDU provides regulated power isolated from the bus to the C\&DH unit, the Scanner Servo Electronics (SSE) in the SBA, and to the Turret Power Regulation Board (TPRB) located in the STA, and main bus power for the Laser Power Supply (LPS) in the STA. Figure 10 shows the converter and power distribution configuration for the PDU. 


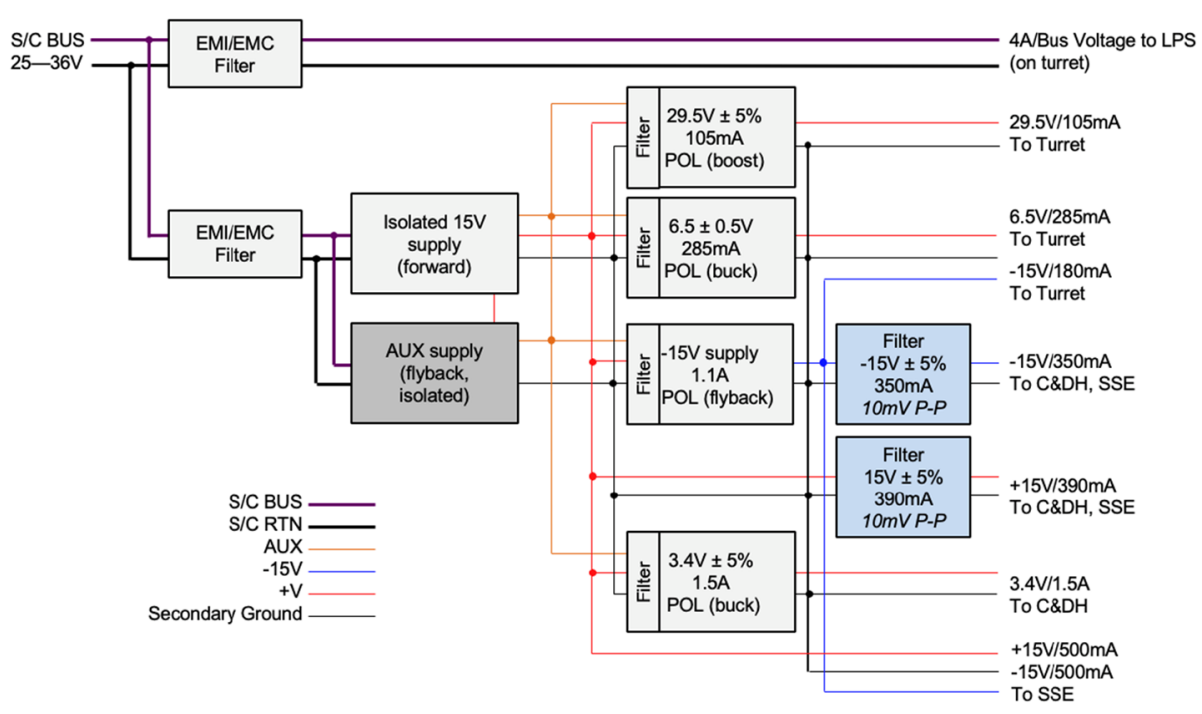

Fig. 10 PDU power distribution schematic

\subsection{Command and Data Handling (C\&DH)}

The block diagram of the spectrometer C\&DH within the body unit is shown in Fig. 11. It incorporates a LEON3 processor running at $50 \mathrm{MHz}$, along with boot ROM (Read Only Memory), 6 MB of nonvolatile MRAM (Magnetoresistive Random Access Memory), and $250 \mathrm{MB}$ of SDRAM (Synchronous Dynamic Random Access Memory), all of which are EDAC (Error Detecting and Correction) protected. The processor interfaces to the spectrometer electronics (SE) via 10 Mbit SpaceWire, and through a ProASIC3 FPGA (Field Programmable Gate Arrays) to the RCE, scanner, and SE discrete signals. Regulated power for the C\&DH is supplied from the PDU.

The processor runs the VxWorks operating system, which is stored along with the application in redundant banks of MRAM. Two copies of both the operating system and application are stored in two banks of MRAM, either of which can be booted based on discrete command from the RCE. There are two rover interfaces (the same as those used on MSL), one to each of two redundant RCEs for commands and telemetry. The RCE interface consists of LVDS clock, data and frame signals. The command interface from the RCE runs at $2 \mathrm{Mbits} / \mathrm{s}$ and the telemetry interface from the $\mathrm{C} \& \mathrm{DH}$ to the RCE operates at $8 \mathrm{Mbits} / \mathrm{s}$.

The C\&DH manages the synchronization of the functions required to perform spectroscopy. Coordination of scanner motion and spectral collection are optimized to improve overall timing of operations. Scanner motion is commanded during the read portion of active spectral collection before proceeding to the next step in the map. Each active spectrum is preceded by a dark collection, where the spectrum is acquired without laser firing, for background removal for ground or flight processing. Under nominal operating conditions, this process runs at $\sim 10 \mathrm{~Hz}$. The maximum number of spectra that can be collected in a single operation is 1296. Results accumulate in the SDRAM and are transmitted to the RCE when commanded.

The C\&DH software includes a suite of algorithms to decimate spectral maps into decisional data sets or to retarget chemical specific regions of interest. Decisional data sets are those data acquired by the instrument which must be downlinked immediately to help 
Fig. 11 Block diagram for the spectrometer command and data handling $(\mathrm{C} \& \mathrm{DH})$ electronics

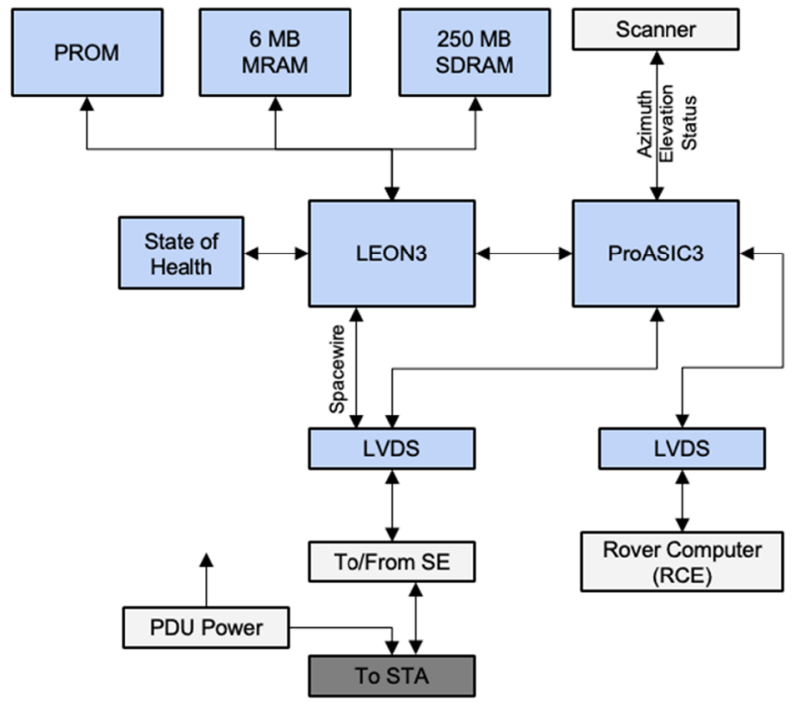

make key decisions for the very next tactical operations plan to be created by the rover team operating on Earth. They can be generated by downlinking laser data only, spectrally binning maps in up to 6 spectral channels, or spatially binning maps to macro pixel(s) based on spatial binning masks stored in NVM or in situ loaded masks. For region of interest selection, initial pre-processing of data is critical to properly retarget chemical signatures. The SHERLOC algorithm suite includes onboard background subtraction, bad pixel removal, laser normalization, and cosmic ray removal. Cosmic rays will cause large spurious spikes obfuscating results and therefore are removed using the statistical analysis method described by Uckert et al. (2019). Preprocessed data are then ranked and positions ordered based on a weighted sum of up to 6 spectral channels. The resulting ranked position list is used to retarget the center to the Nth ranked position. Algorithm parameters are stored in nonvolatile MRAM and can be changed by command. Single data sets can be reprocessed for multiple times for targeting different chemical features as time permits in the operations timeline.

\subsection{Scanner Servo Electronics (SSE)}

The SSE is a part of the scanner subsystem and serves as the interface between the scanner and the control (C\&DH) and power (PDU) systems. The SSE converts control and power signals for distribution to the scanner subsystem located on the turret and positional feedback to the scanner through the servo control loop.

\subsection{Digital Electronics Assembly (DEA)}

The shared DEA (Fig. 24b) performs all interfacing functions with the rover to operate the WATSON and ACI imagers. The DEA, described previously by Edgett et al. (2012) and Malin et al. (2017), contains flight software to operate the imagers and perform onboard data compression (including focus stacking). The DEA hardware is equivalent to that of the Mars 2020 Mastcam-Z DEA (Bell et al. 2020, this journal) and has 128 megabytes of SDRAM and 8 gigabytes of non-volatile NAND flash memory for data storage. 
Fig. 12 The SHERLOC turret assembly with the location of the primary subsystems outlined. At the left, the ACI dust cover is open and its Internal Calibration Target can be seen on the inside of the cover as well as the UV and white LEDs. WATSON is present on the right; its transparent dust cover is closed

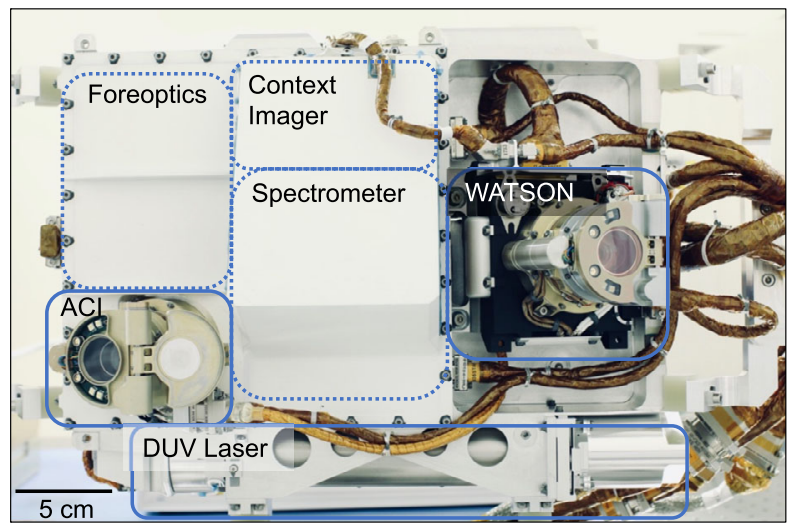

\section{SHERLOC Turret Assembly (STA)}

The SHERLOC Turret Assembly houses the optical, mechanical, thermal and instrument head electronic hardware for the Spectroscopy and Imaging subsystems (Fig. 12). In addition to the optics, the STA contains only critical electronics that are required to be in proximity to hardware. The spectroscopy subsystem electronics includes the spectrometer electronics and turret power regulation board (SE/TPRB), the spectrometer CCD (SCCD) and control electronics, the scanner sensor feedback electronics (SDE), and the high voltage power supply to run the DUV laser (LPS). The imaging electronics on the STA include the context imager FPA (CHE), and the WATSON FPA (WCHE). In addition, to minimize impacts and resources to the flight system, the ACI and WATSON imagers have a command switchable interface for the command, data, and telemetry, and simultaneous power signals from the DEA via a multiplexer (MUX). The STA mass is $6.83 \mathrm{~kg}$ and has an enveloped volume of $352 \mathrm{~mm}$ x $176 \mathrm{~mm}$ x $276 \mathrm{~mm}$ excluding connectors and cabling.

\subsection{SHERLOC Spectroscopy Subsystem Optical Design}

SHERLOC science goals require a DUV laser to excite a target, and a spectrometer that senses the Raman and fluorescence spectral regions as it measures the laser-induced spectral signatures from that target. In addition, the motorized objective lens and the context imager of the ACI provides a means to focus the laser and spectrometer and generate images at a pixel scale of less than $1 / 10$ the laser diameter. These three co-boresighted optical paths comprise the SHERLOC optical system (Figs. 13 and 14).

Guided by the focus position established by acquisition of the ACI image, SHERLOC uses a tip/tilt fine steering mirror to step a focused DUV laser spot across the target, generating Raman scatter and fluorescence emission from each step position. SHERLOC's laser emission starts in its neon-copper laser, where the primary emission at 248.6-nm is output as a Laguerre-Gaussian beam with an $\mathrm{M}^{2}$ of 34 . Also emitted is an orange spontaneousemission spectrum from the glow discharge and a number of weak laser lines, including a particularly strong, non-lasing line at $252.9 \mathrm{~nm}$. These longer-wavelength spectral features must be attenuated significantly, so that they do not influence the spectroscopy. To this end, the first optical elements after the laser's output coupler are steep edge laser-injection filters (LIFs) (Semrock). These filters strongly (>99.995\%) reflect light less than $251 \mathrm{~nm}$ and transmit (on average, about 97\%) longer wavelengths. A series of three such filters reflect the 248.6-nm line and attenuates longer wavelengths, while turning the laser beam by 


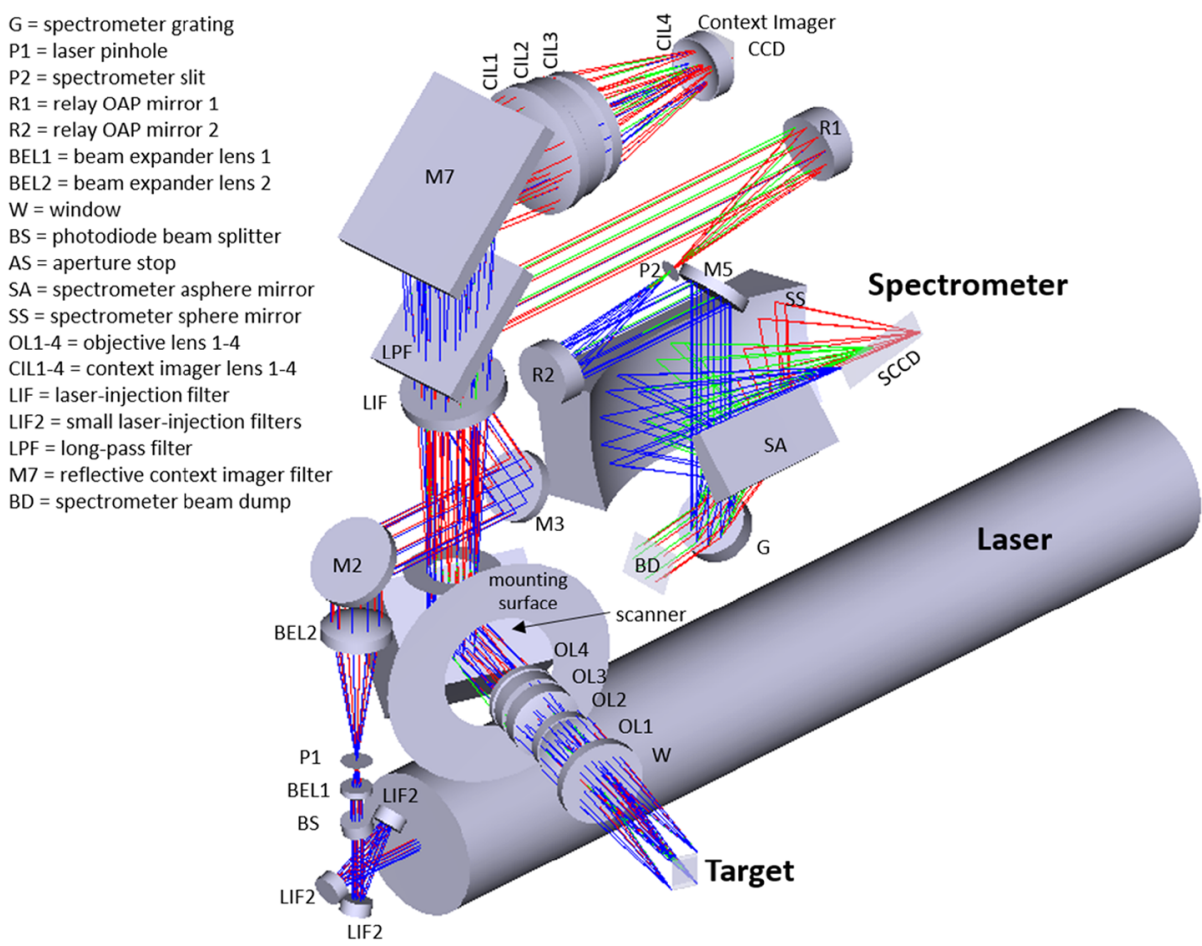

Fig. 13 Ray trace of the SHERLOC optical path

$90^{\circ}$ into the main structure of the SHERLOC instrument. Just before entering SHERLOC, an uncoated fused silica beamsplitter reflects a tiny fraction of the laser signal onto a silicon carbide photodiode (sglux SG01XL). This detector acts as a reference measurement that allows continual assessment of the laser's pulsed output. The laser signal that transmits through the beamsplitter is then expanded by two aspheric lenses lens that collimate the beam to its full diameter, critically filling the instrument's $12.5-\mathrm{mm}$ aperture stop. The collimated output of this aspheric beam expander then reflects off of two mirrors that are coated to reflect the $248.6 \mathrm{~nm}$ laser line (M2 and M3), then off the LIF edge filter, off the scanner mirror, and out the common-path ACI objective, which transmits nearly $90 \%$ of the laser signal and focuses the laser to an approximately $100-\mu \mathrm{m}$ spot on the target. The scanner is then actuated to articulate the laser spot across the target field, thereby inducing a Raman and fluorescence signal at every desired pixel on the target's context image.

The target's laser-induced Raman and fluorescence signals are collected along the same optical path back into the instrument, through the ACI objective and off the scanner mirror. However, this excited signal has a slightly longer wavelength than the exciting laser line, so it is transmitted through the LIF and along SHERLOC's spectrometer optical path. The next filter is a long-pass filter (LPF) (Semrock) that reflects the spectral signal onto an offaxis paraboloidal (OAP) mirror. This OAP mirror focuses the spectral signal through the spectrometer's slit, a 100-by-200- $\mu \mathrm{m}$ laser-drilled aperture. This slit limits the spectroscopy aperture. The spectroscopy signal then continues to be collimated by another OAP mirror, after which it is reflected off a fold mirror (M5) and directed onto the instrument's diffraction grating. This unique grating was written via electron-beam lithography (EBL) at the JPL Microdevices Laboratory (MDL) at a very high groove density of 4200 grooves $/ \mathrm{mm}$. 


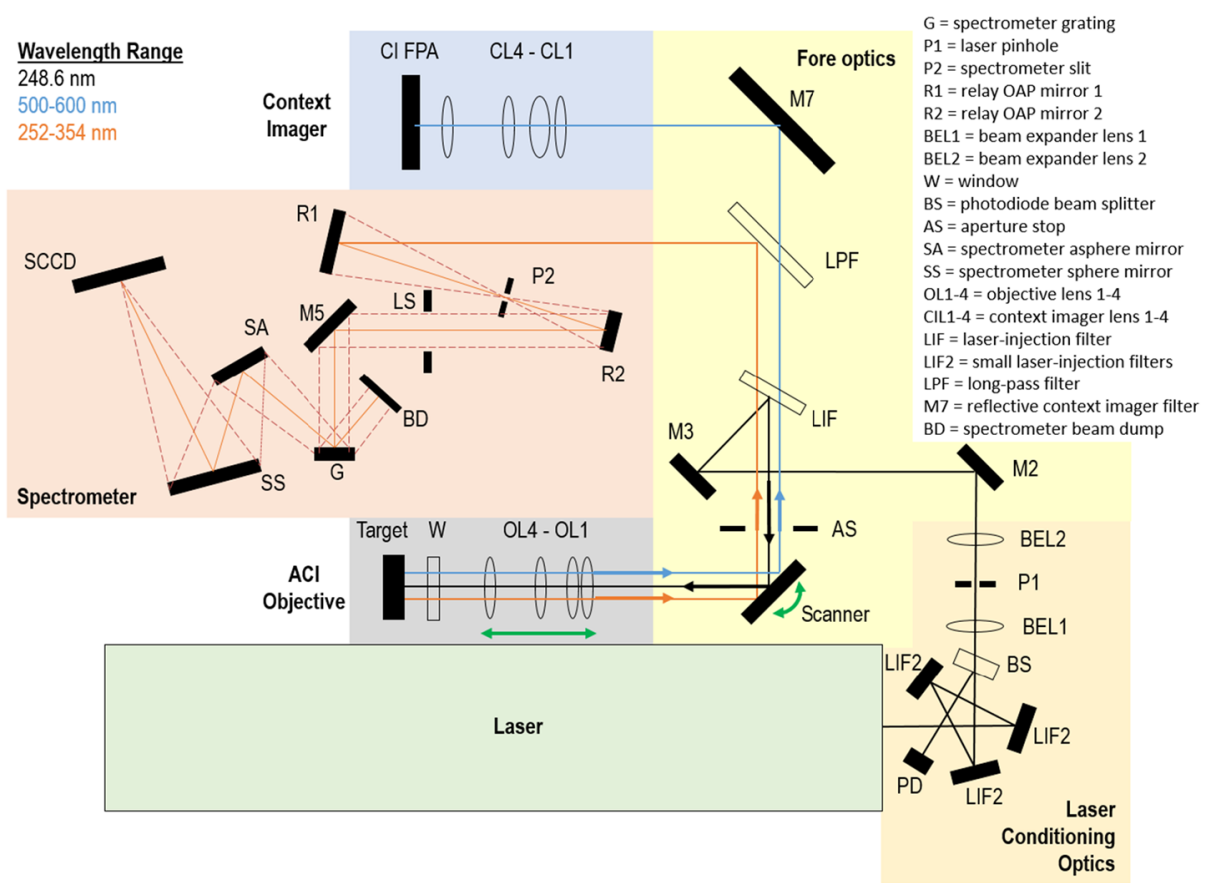

Fig. 14 Block diagram of the SHERLOC optical path. The LIF/LIF2, LPF were provided by Semrock, M7 was from Alluxa, the beam dump, grating, aperture stops were fabricated at JPL and coated by Aktar. The remaining lenses and curved mirrors were custom fabrications to meet the deep UV surface figure and RMS requirements with margin (provided by Optimax)

The zeroth-order diffraction is dumped into an angled port (which was used to aid instrument alignment and designed to minimize stray light), and the first-order beam is diffracted with high efficiency (nearly $60 \%$ ) onto a convex aspheric mirror, and then onto a concave spherical mirror that focuses the spectral signal across the long axis of the 512-by-2048 pixel e2v 42-10 SCCD.

The SHERLOC spectroscopic analysis takes place on the SCCD. The target's spectral signal is read out in three binned regions, one for the Raman signal from 253 to $274 \mathrm{~nm}$ and two for the fluorescence regions that range up to $354 \mathrm{~nm}$. In the Raman region, $16.2 \mathrm{~nm}$ of the spectrum are diffracted across each of the SCCD's 13.5- $\mu$ m pixels. The instrument samples a narrow line source (e.g., the 253.65-nm $\mathrm{Hg}$ line) with an instrument line function of $<5$ pixels Full Width at Half Maximum (FWHM) in the Raman region. The optical design specifies a spectral resolution of $310 \mathrm{pm}$ in this region, and the performance measured was $269 \mathrm{pm}$. Spectra are diffracted across the SCCD as a curved line, called a spectral smile (Fig. 15). To optimize for the photon-starved Raman signals, the smile is registered to the pixel grid by clocking the SCCD by $\sim 9^{\circ}$. This orients the Raman spectral region along pixel rows and minimizes the number of rows binned to generate the spectrum. The fluorescence spectral region curves across the SCCD into the opposite corner. As it is many orders brighter than Raman, binning more rows has a negligible effect on SNR. Data are read in a binned, columnar fashion, since signal is distributed so that wavelength corresponds to SCCD column.

To mitigate ghost reflections and other stray light within the instrument, a physical aperture shaped like the spectral smile is located just before the SCCD. In addition, a baffle 


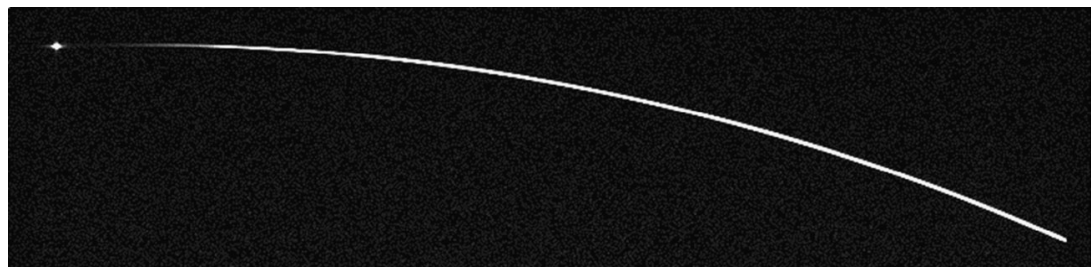

Fig. 15 An example diagnostic two dimensional read of the SCCD for one point on the resolution target that shows the spectrometer "smile" pattern from DUV laser illumination of Bk7 glass

is located around the grating periphery to obscure edge effects and other artifacts of the multilayer EBL process. All aperture masks in SHERLOC, including the laser pinhole, instrument aperture stop, spectrometer slit, grating mask, and SCCD smile mask are coated with the same Acktar black broadband-absorbing coating.

\subsubsection{Deep UV Laser}

Deep UV Laser Overview The SHERLOC NeCu DUV laser tube was manufactured by Photon Systems, Inc (PSI). A commercial-off-the-shelf (COTS) laser design manufactured by PSI was modified specifically for the SHERLOC instrument to meet launch, cruise, landing, extreme operating and non-operating ambient temperature, and vibration conditions of a Mars rover mission.

For accurate accumulation of Raman and fluorescence spectra on Mars, the stability of the laser emission linewidth and wavelength are essential. The SHERLOC NeCu laser emits at a wavelength of $248.6 \mathrm{~nm}$ and, since it is an electronic transition, has a line width and stability less than $0.07 \mathrm{~cm}^{-1}$. The laser is operational without survival heaters or any temperature regulation or control at ambient temperatures from $-135{ }^{\circ} \mathrm{C}$ to $+70{ }^{\circ} \mathrm{C}$. In addition to the ambient temperature requirements, the $\mathrm{NeCu}$ laser was subject to several additional mandatory requirements including:

(1) emission wavelength below $250 \mathrm{~nm}$ to enable spectral separation of the Raman and fluorescence spectral emission regions;

(2) beginning of life output energy $\geq 9 \mu \mathrm{J}$ per pulse with pulse repetition rate of $80 \mathrm{~Hz}$ in $40 \mu$ s-wide pulses with a peak power less than about $200 \mathrm{~mW}$ to avoid photochemical or thermal damage of organic target materials;

(3) stable emission linewidth and position and independent of ambient temperature, to enable accurate Raman spectroscopy without added system size and mass associated with the need for additional spectral calibration components;

(4) unpolarized to enable Raman detection of crystalline materials of unknown orientation;

(5) insensitive to hard radiation (rad-hard);

(6) able to survive the severe shock and vibration expectations through the entire mission envelope, particularly including launch, landing, and during drilling/coring operations;

(7) low mass and volume $\left(<280 \mathrm{~cm}^{3}\right.$ and $\left.<450 \mathrm{~g}\right)$; and

(8) start-up time $<1 \mu$ s to eliminate power consumption before use.

The $\mathrm{NeCu}$ laser is a transverse discharge sputtered $\mathrm{Cu}$ hollow cathode laser which was first demonstrated in 1974 at the University of Budapest (Csilliag 1974) and Colorado State University. The transverse excited hollow cathode sputtered copper laser tube is a simple, coaxial, robust design with grounded anode invar tube body and internal construction consisting 
Fig. 16 The SHERLOC $\mathrm{NeCu}$ flight laser. For scale, the laser with the end caps is $30 \mathrm{~cm}$ in length. The green emission in the photograph is from an alignment laser

Fig. 17 Cross-section diagram of $\mathrm{NeCu} 25$ hollow cathode laser gain region
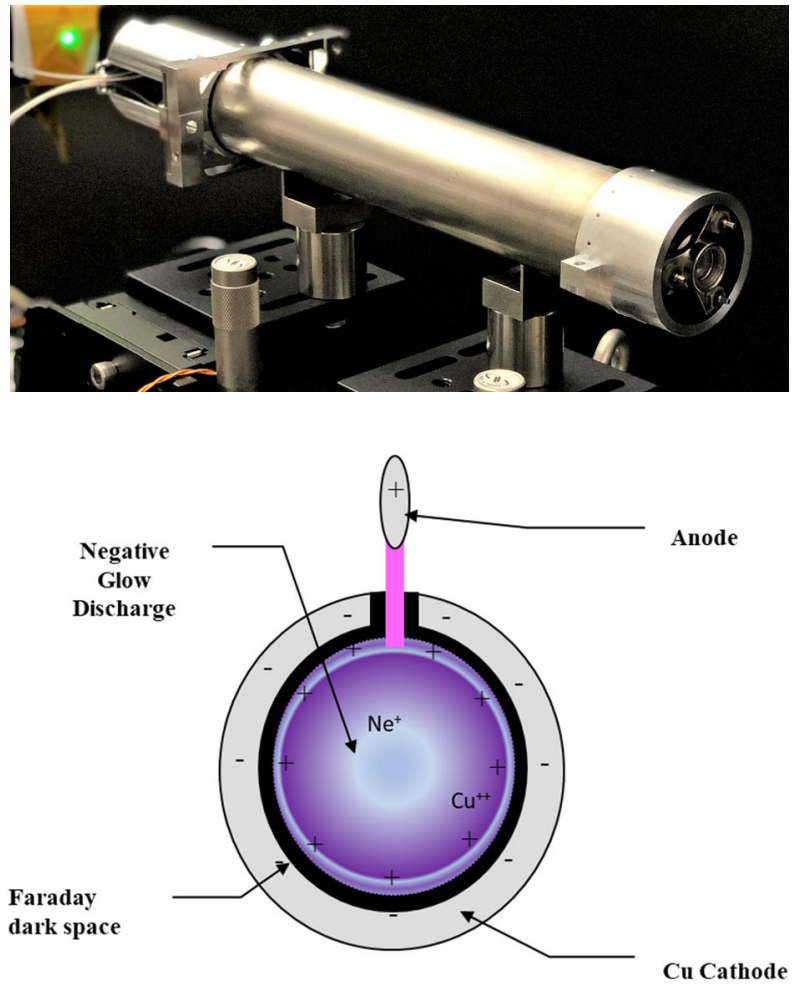

mostly of invar, beryllium oxide, titanium, OFHC (Oxygen-Free, High thermal Conductivity) copper bore, deep UV grade fused silica laser mirrors and windows hermetically sealed with indium and filled with a neon buffer gas. This all-metal, welded, and brazed construction leads to a robust traveling wave tube (Fig. 16). A photograph of the $\mathrm{NeCu}$ laser as mounted on the SHERLOC STA is shown in Fig. 8.

Hollow Cathode Laser Figure 17 shows a cross-section view of the structure of the laser. When a negative voltage between $-550 \mathrm{~V}$ and $-800 \mathrm{~V}$ is applied to the laser, it causes an electrical breakdown of the low-pressure neon buffer gas $(\sim 12$ Torr $)$ where the discharge avalanches to a stable operation at a sustaining voltage of $\sim 380 \mathrm{VDC}$ and current between 20-30A. This process takes $1 \mu$ s after application of voltage. The electrons are accelerated inwardly across the Faraday dark space, into the negative glow region undergoing multiple ionizing collisions with neon $(\mathrm{Ne})$ neutrals and producing neon ions $(\mathrm{Ne}+$ ). This production of $\mathrm{Ne}+$ results in an initial bombardment into the $\mathrm{Cu}$ cathode wall via the acceleration back through the Faraday dark space. These highly energetic $\mathrm{Ne}+$ ions begin generating neutral $\mathrm{Cu}$ atoms via the sputtering process that is enhanced as $\mathrm{Cu}+$ ions are created in the negative glow region and, in turn, are themselves are accelerated into the $\mathrm{Cu}$ wall, efficiently creating even more $\mathrm{Cu}$ atoms (de Hoog et al. 1977; McNeil et al., 1975 and 1976). These $\mathrm{Cu}+$ wall collisions combined with the relatively low $\mathrm{Cu}$ work function produce secondary electrons bootstrapping the process. These sputtered $\mathrm{Cu}$ atoms diffuse back into the negative glow region and are autoionized creating the highly excited upper laser state via charge transfer reaction with the $\mathrm{Ne}+$ metastable ions. One of the unique features of this laser is the (annular ring) Laguerre-Gaussian laser mode which is a result of the nonuniform distribution of 


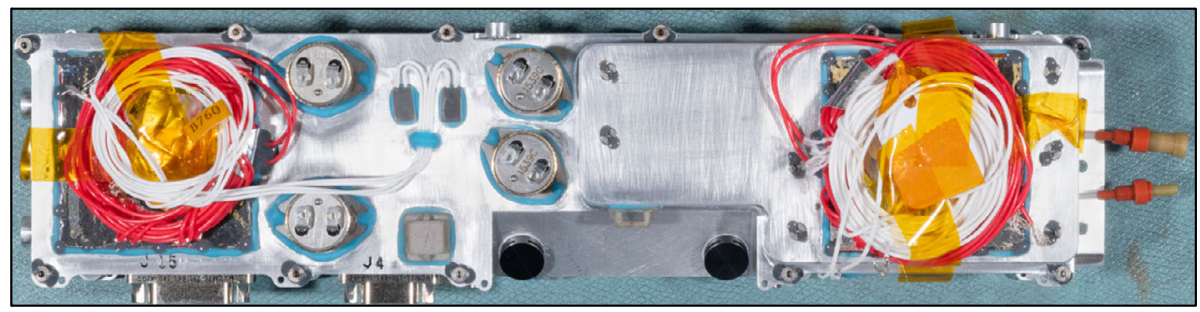

Fig. 18 Photograph of the $\sim 10 \mathrm{~cm}$ SHERLOC LPS

$\mathrm{Cu}++$; higher density at or near the wall of the $3 \mathrm{~mm}$ copper bore produces a smoke ring like laser gain region. The process, including stabilization of the copper ion density in the hollow cathode, takes less than $10 \mu \mathrm{s}$, independent of ambient temperature.

The fundamental lifetime limit of the hollow cathode laser of the $\mathrm{NeCu} 25$ type is about 500 million laser pulses at current levels of 25 to 30A, due to erosion of the copper cathode. For SHERLOC, the required number of pulses is a convolution of the instrument throughput and collection efficiency. Assuming $9 \mu \mathrm{J} /$ pulse, the required number of pulses to be available for use on Mars is $\sim 21 \mathrm{M}$. Given the $>14 \mu \mathrm{J} /$ pulse laser, the SHERLOC flight laser will easily enable acquisition of $>2.7$ million spectra, which is $>7$ times the lifetime of the prime mission.

Tube Construction and Testing The SHERLOC NeCu laser is based on the PSI COTS version, the commercially available NeCu30 model. Although, early during instrument development, a COTS laser was demonstrated to survive exposure to liquid nitrogen temperature $\left(-195^{\circ} \mathrm{C}\right)$, there were mechanical design and process changes to ensure performance under fully Mars-like conditions. The design and construction of the laser evolved as knowledge from testing of previous generations of the laser were incorporated into the designs. As the feedback between design, construction, and testing continued, the rigor of each test increased until we exceeded the nominal SHERLOC operational scenarios (Survey and Detail - see Sect. 10) for the laser, ensuring good working margin.

The CTE (Coefficient of Thermal Expansion) between materials was tailored to reduce stress and deformation of the laser bore and mirrors leading to stable performance over the $200{ }^{\circ} \mathrm{C}$ temperature range the laser would experience. Invar 36 is the primary construction material for the laser tube (outer body) and the mirror mounts. Within this, copper, beryllium oxide, titanium, fused silica, and indium are key materials sealed hermetically in the tube body. Assembling titanium to Invar resulted in the development of a unique, resistive, eutectic TiCuSil braze weld as well as a unique Tungsten Inert Gas (TIG) CuSil braze of Invar to Invar.

Mirror stability is critical to maintaining optimal laser output. A passive Ti6Al4V spring mirror mount was designed to mount into the Invar optic assembly. Hermetically sealing the laser tube used a less mechanically critical fused silica window to Invar seat with an indium seal. This stabilized the laser output over a wide mechanical and thermal range.

To maintain mirror alignment with respect to each other and the gain media within the tube, the laser mirrors are in Moore mounts as part of the laser end-flange. The end flange incorporated a two-part design that was electron-beam welded not only sealing the two halves together but also providing a single convoluted bellow to enable coarse and fine alignment of the mirror without imparting stress to the mount.

In addition to mechanical modification, laser processing was significantly modified compared to the manufacture of a COTS laser. Some methods were necessary to enable laser 
integration into the constrained volume of the STA while others were used to increase mechanical stability over the required thermal and vibrational environments. The volume constraints included a custom pinch-off method to form a physically short, but high-integrity vacuum seal. To increase material and mechanical stability, a bake-out for organic cleaning and invar stabilization thermal cycles were implemented. In addition, staking methodologies were implemented to ensure mirror mount stability.

To assess the laser stability over the laser life, a number of lasers were fabricated to conduct full, accelerated, life tests. These were performed in both ambient conditions as well as operational and non-operational environments. Laser performance was verified and measured in test-as-you-fly (TAYF) operating conditions, including vibration and thermal specifications with some test lasers taken through entire lifetimes to determine performance over mission lifetimes. This is discussed in more detail in Sect. 8.2

\subsubsection{Laser Power Supply (LPS)}

SHERLOC's LPS provides high-voltage, high-current pulsed power to fire the DUV laser (Fig. 18). It operates directly off of the rover bus at $25 \mathrm{~V}-36 \mathrm{~V}$ and provides current pulses of duration, frequency and amplitude controlled by an external $5 \mathrm{~V}$ logic signal. The pulse repetition rate can be specified in the $10 \mathrm{~Hz}-160 \mathrm{~Hz}$ range depending on operational need. The pulse amplitude is commandable to $30 \mathrm{~A} / 25 \mathrm{~A} / 20 \mathrm{~A} / 15 \mathrm{~A}$ as a means to adjust laser energy at the target. The pulse duration is also commandable between a range of $20 \mu$ s to $40 \mu \mathrm{s}$. The LPS comprises two main subcircuits: a $28 \mathrm{~V}$-to-550 V isolated DC-to-DC converter using the flyback topology operating at $50 \mathrm{kHz}$ and two wide-band (fast response) current amplifiers in parallel operating off of the $-550 \mathrm{~V}$. As such, the output of the LPS is referred to as a compliant current source with a compliant voltage of $-550 \mathrm{~V}$. The output of the LPS is equipped with an arc protection circuit which detects a drop in the laser voltage (output voltage of LPS) below a certain voltage while providing the current pulse and terminates the pulse within $2 \mu$ s to protect the laser. This protection circuit also protects against inadvertent short-circuit that could be present in the output cable to the laser.

During the development of the LPS, the output capacitors were removed and placed on a separate board within the same housing compartment of the LPS. Electrically, the routing of the power and sense wires from the capacitor bank to the mother board were designed to resolve noise and other interference issues. Mechanically, wire lengths connecting the capacitor board to the main board were minimized to reduce noise, while still allowing the unit to be handled by the mechanical integration team. The ability to rework the unit was paramount when environmental testing revealed a need for conformal coating updates to mitigate concerns of corona discharge. After much investigation and testing, the team mitigated corona by increasing the surface area of the high voltage components by adding a series of conformal coating layers to the board.

The traditional voltage for igniting the COTS laser requires $-900 \mathrm{~V}$. This value is used in order to eliminate misfires and lower output laser energy. However, flight qualified components at that voltage were not available due to operating environmental conditions. After testing demonstrated that the laser could fire consistently with $-550 \mathrm{~V}$ at all operating temperatures and internal laser pressures (simulating beginning and end of laser life) the LPS was designed to operate at this voltage, systems were designed to monitor misfires and laser output voltage. A 1200 V MOSFET (Metal Oxide Semiconductor Field Effect Transistor) with sufficient voltage and current rating made by Fairchild was qualified and selected for the output amplifier. This MOSFET was selected after the selection (up-screening) of a plastic package using the proper testing procedure prescribed to the Parts Engineering group by the LPS engineers. 
Fig. 19 Block diagram of the Spectrometer Electronics

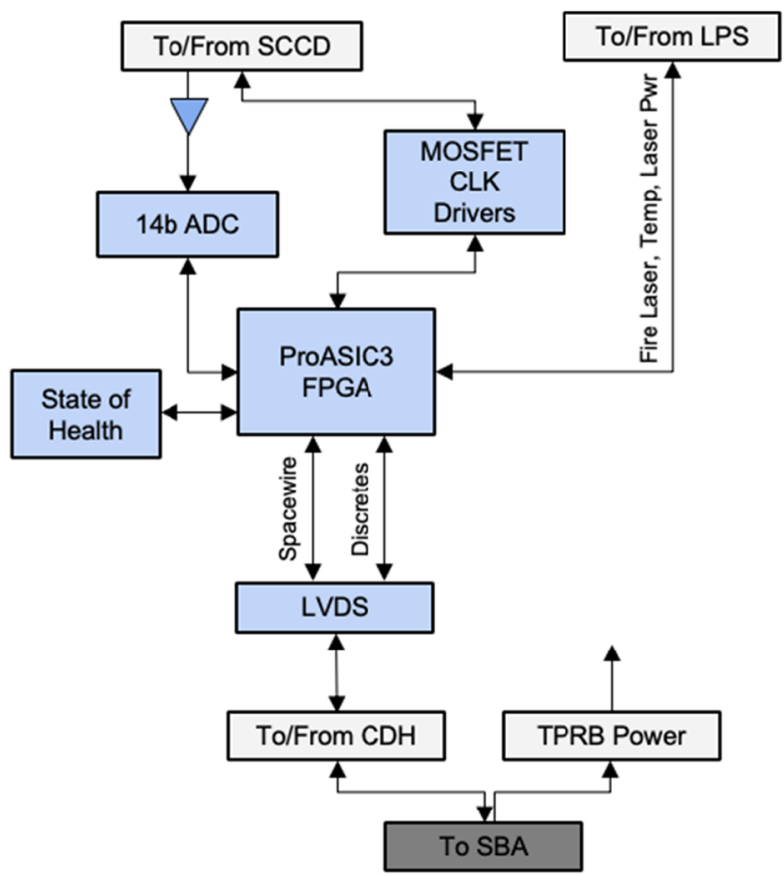

\subsubsection{Spectrometer Electronics (SE)}

The Spectrometer Electronics (SE) module has four electrical interfaces: the detector (CCD module), the laser power supply (LPS), the command and data handling (C\&DH) in the body via the harness down the robotic arm, and the TPRB for power, as shown in Fig. 19.

The SE receives its power from the TPRB that interfaces from the PDU to the SE to provide linearly regulating power singles for the critical functions including $3.3 \mathrm{~V}, 5 \mathrm{~V}$, $+/-12 \mathrm{~V}$ and $26.4 \mathrm{~V}$. The addition of the TPRB was led by efforts to minimize the impacts of arm flex cable voltage drop and to minimize mass on the turret. SHERLOC used MSL heritage design for TRCE chip-on-board ( $\mathrm{CoB})$ technology in the TPRB leading to a $12.7 \mathrm{~g}$ power board.

The SE generates the bias voltages and clocks for the detector, conditions the spectrometer CCD output, and samples the detector signal. The SE implements a correlated double sample technique as described by de la Torre and Albendea (2018) and then samples the signal with an analog to digital converter (ADC). The ADC, a 14 bit AD9240 from Maxwell/DDC, is the same as that used on the MSL ChemCam.

During the CCD readout process, samples are transmitted in real-time to the C\&DH in the body via a $10 \mathrm{Mb}$ SpaceWire link, no memory is required in the SE. The C\&DH interface also includes discrete LVDS signals for reset, laser fire, and integration handshaking. The integration request and acknowledge allow the $\mathrm{C} \& \mathrm{DH}$ to synchronize the detector readout and clearing with the laser firing and scanner motion. The SE also collects telemetry on laser output energy and health, along with a variety of temperatures, which are also sent via SpaceWire to the C\&DH.

The SE will experience dramatic temperature swings during a sol on Mars. When powered off, there is no survival heat for the SE. Several strategies were used to ensure that the electronics will operate over its design lifetime. The printed circuit board is clamped rather 
than fastened into position, allowing it to expand and contract without inducing excessive stress. Several components are mounted in a "dead bug" fashion and flexible leads connect them to the printed circuit board. The FPGA is in a column grid array package, which has flexible leads that do not fracture as the board expands and contracts, and the corners of the package are staked. A warm-to-use heater is used to bring the assembly up to $-80{ }^{\circ} \mathrm{C}$ before it is powered on. Finally, package qualification and verification (PQV) testing equivalent to three lifetimes was performed on a test article to demonstrate that the design can survive the environment.

\subsubsection{Spectrometer Charged Coupled Detector (SCCD)}

The SCCD is an e2v CCD 42-10 device that has an active area of 512 x 2048 pixels with an area of $13.5 \times 13.5 \mu \mathrm{m}$ each. The image area is approximately $28 \times 7 \mathrm{~mm}^{2}$ and has an ultraviolet enhanced coating. To withstand extreme temperature cycling, the leads of the detector were replaced with short, flexible wires and relief loops that will flex with the temperature changes rather than fracture.

The electronics are capable of reading the array in the 2D format for diagnostics; however, under normal operating conditions, on-chip vertical integration is used and the spectral vectors are read from the device.

The size and location of the vertical summing regions is configurable by command. The vertical integration is configured for three regions using skip and sum parameters written by the processor into the SE FPGA registers, as shown in Fig. 20. This flexibility allows the instrument to adapt to any modest shifts in the spectrometer pattern encountered throughout the mission due to aging, shock or vibration. The objective is to integrate as many pixels containing light from the spectrometer while leaving out as many unilluminated pixels as possible to maximize the signal to noise ratio (SNR). Performing integration on the detector improves the SNR by only adding read noise to summed pixels rather than each pixel, so the effective read noise is reduced by the number of pixels integrated vertically. It also has the benefit of accelerating the readout process.

Faster readout reduces operation time and reduces the dark current contribution, which is a function of temperature of the detector and time. Higher temperatures and longer time increase the total dark current. The detector is mounted on a phase change material (PCM) with a melting point of $-29{ }^{\circ} \mathrm{C}$. The PCM freezes in the ambient Martian environment when the instrument is not in use, and then keeps the detector at $-28{ }^{\circ} \mathrm{C}$ for the $>60$ minutes of operating time.

The SCCD clocks and bias voltages are driven from the SE module, and the SCCD output is differentially driven to the SE and sampled.

The SCCD has three vertical and three horizontal clocks that are driven from the SE module. All of the clock speeds, widths, and phasing are configurable with parameters sent to the instrument via command. The typical vertical clocks are $55 \mathrm{kHz}$, and the maximum horizontal clock rate is $400 \mathrm{kHz}$. The maximum horizontal rate is governed by the maximum ADC clock rate and the digital correlated double sampling scheme utilized.

The detector has two important contributions to noise, read noise, and dark current. Read noise is a property of both the detector itself and the readout circuitry, while dark current is a property of the detector and its environment, specifically temperature and radiation.

Significant effort was invested to minimize the read noise contribution. One design feature incorporated is configurable gain, implemented on the SE. The gain can vary between high and low, with high employed typically in region 1 to capture the Raman portion of the spectrum, and low gain in the fluorescence regions 2 and 3. In high gain mode, the read 


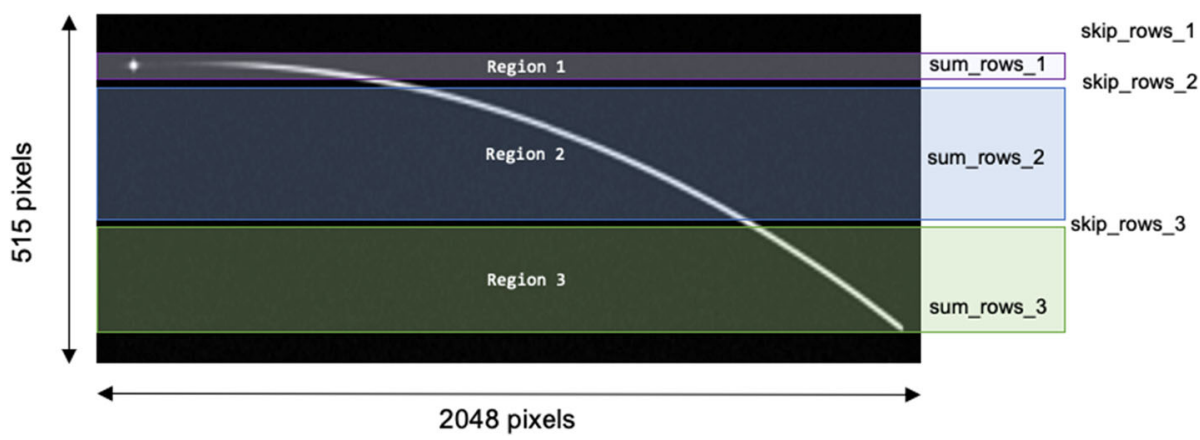

Fig. 20 Illustration of the SCCD detector array overlayed with the spectrometer "smile" pattern and the on-chip integration patterns

noise is $<6 \mathrm{e}^{-} \mathrm{rms}$, with a conversion gain of $0.46 \mathrm{e}^{-} / \mathrm{DN}$ (digital number) at a readout rate of $400 \mathrm{kHz}$. In this mode, the ADC saturates at $\sim 28 \mathrm{ke}^{-}$. In low gain mode, the read noise is $\sim 10 \mathrm{e}^{-}$rms, with a conversion gain of $2.35 \mathrm{e}^{-} / \mathrm{DN}$. In this mode, the ADC can detect the single pixel full well saturation of the SCCD at $\sim 101 \mathrm{ke}^{-}$. With integration performed on the SCCD, output can saturate the ADC, as the summing register has $\sim 1.5 \mathrm{x}$ the capacity of a single pixel. By varying laser energy and the number of laser pulses per spectrum, the signal amplitude can be kept in range of both the SCCD and the ADC.

Dark current mitigation involved "cherry picking" the flight detector from multiple candidate devices to get the detector with the lowest noise at the beginning of life. Radiation effects have a significant impact on dark current in the SCCD. Both solar and cosmic radiation contribute to the environment during cruise and surface operations, but the primary mechanism for radiation effects on the Mars 2020 rover is due to the neutrons from the Multi-Mission Radioisotope Thermoelectric Generator (MMRTG). The neutrons from this power source induce displacement damage in the lattice structure that result in an increase in dark current (Janesick 2001). Ionizing dose can also result in inversion voltage shifts. This was not observed at the anticipated dose levels. As a mitigating precaution, the design maintains a substrate voltage above $9 \mathrm{~V}$. Radiation testing devices from the same lot as the flight detector indicate that, at $-28{ }^{\circ} \mathrm{C}$, the SCCD will have a dark current of $<1 \mathrm{e}^{-} / \mathrm{pixel} / \mathrm{s}$ at the beginning of life and approximately $50 \mathrm{e}^{-} / \mathrm{pixel} / \mathrm{s}$ at the end of the mission.

\subsubsection{Scanner Subsystem}

To raster scan the laser/spectrometer field of view (FOV), SHERLOC uses a fine steering mirror manufactured by Left Hand Design Corporation. The mirror is controlled with a combination of fixed magnets and voice coil electromagnets driving the lightweight mirror mounted on flexures. The assembly has embedded position feedback sensors connected to the scanner demodulation electronics (SDE) and the Scanner Servo Electronics (SSE) that closes the loop with an analog control system with very high bandwidth. To specify a position, the $\mathrm{C} \& \mathrm{DH}$ provide a voltage for the azimuth and elevation axes and the control loop drives the mirror position so that position sensor outputs match these voltages. This system provides the capability to steer the beam across the $7 \times 7 \mathrm{~mm}$ work area. During instrument testing, both steps and repeatability of $<1$ micron in the target were demonstrated, meaning that arm stability is expected to set the limit on the instrument's ability to revisit the same spot during a given observation. During ground testing, the az/el input voltages were 


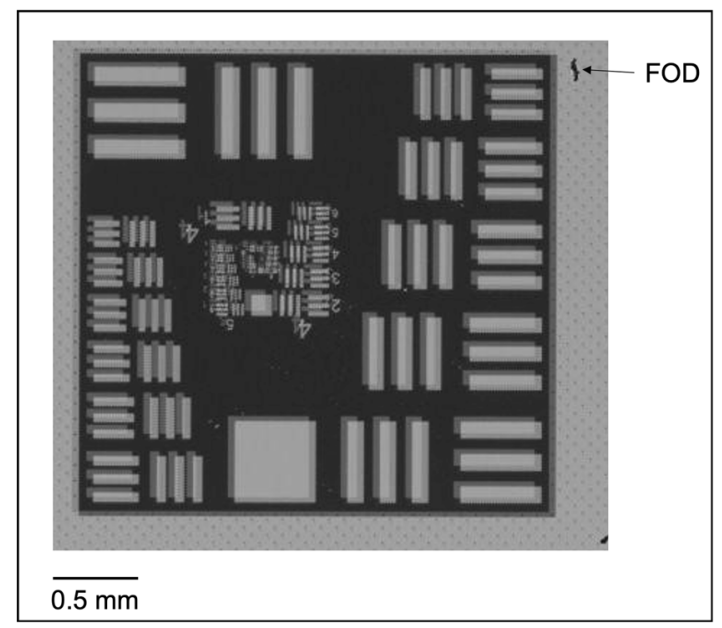

Fig. 21 Illustration of SHERLOC scanner motion. This image was captured using the engineering model SHERLOC ACI during a test of the scan mirror. The exposure duration of this image was $200 \mathrm{~ms}$ and the scanner shifted the image $\sim 70$ microns during this integration time. The result is the superposition of two images of the image target before and after the scanner move. The fact that no streaking can be seen, and that both images have similar sharpness, demonstrates that the scanner motion and settling times are both extremely fast compared to the typical exposure duration of the camera. Note there is FOD (Foreign Object Debris) on the engineering model ACI detector. This can be determined as FOD because the feature does not shift between scanner positions

calibrated to $\mathrm{XY}$ positions in the work space so that scan patters could be generated that sampled the target in even steps (Fig. 21).

\subsection{SHERLOC Imaging System}

\subsubsection{Imaging Electronics}

The imaging subsystem includes two cameras, the ACI and WATSON. A Multiplexor unit (MUX) (Fig. 24c) mounted on the STA, is shared by the WATSON and ACI to allows switching of camera head operation between them. The MUX is connected by cabling on the rover's robotic arm to the DEA inside the rover body. The MUX allows the DEA and cabling to be shared, cutting in half the mass and volume of cabling and DEAs needed to support the two imaging subsystems. This architecture means only one of the two camera heads (ACI and WATSON) can be operated at a given time. To simplify its design, the MUX has no power switching, so both cameras are powered whenever power is enabled. The inactive camera is commanded into a low-clock-rate mode to reduce total power usage. Regulated $15 /-15 \mathrm{~V}$ power from the DEA is passed through directly, but the MUX has internal boost regulation for the $5 \mathrm{~V}$ power. This was required because the additional power of two camera heads caused unacceptably large voltage drops due to cable resistance in the long interconnecting flex cable between the DEA and the MUX. The only other active components in the MUX are low-voltage differential signal (LVDS) drivers and receivers, which are enabled and disabled by a small amount of control logic. The active camera is selected by the state of the data line in the inactive period between command frames, and sampled at the beginning of each command frame. 


\subsubsection{Autofocus and Context Imager (ACI)}

SHERLOC science goals require an image to provide texture and morphology context for SHERLOC spectra. With the addition of WATSON images of the same target, the ACI plus WATSON enable location of each SHERLOC spectral position within the broader context of all of the data acquired by Perseverance's cameras, and even tie these to orbiter imaging system views of Mars. This co-registration capability was a Mars 2020 mission requirement and provides a means to correlate data from SHERLOC with data from PIXL, SuperCam, and Mastcam-Z multispectral observations.

ACI, co-boresighted with the SHERLOC spectroscopy subsystem, is a high spatial resolution grayscale camera that serves two vital purposes; it focuses the spectrometer laser to enable spectral mapping over a $7 \times 7 \mathrm{~mm}$ area, and it acquires $10.1 \mu \mathrm{m} /$ pixel context images (or $<2 \mathrm{~Hz}$ video frames) of the area investigated by the spectrometer. It can also be used as a $10.1 \mu \mathrm{m} /$ pixel camera without operating the spectrometer.

When SHERLOC is operated to acquire spectroscopy data, the initial step is to obtain an image of the science target using the ACI. Focusing the ACI to acquire this image is the first step toward focusing the laser. Reflected light scatters off the target and enters SHERLOC through the ACI Objective (Figs. 13 and 14). The Objective collimates light into the SHERLOC optical system, after which it reflects off a scanner mirror, through the instrument's aperture stop, and through two long-wavelength transmission edge filters that control the instrument's spectroscopy observations. The mirror (M7) (Alluxa) immediately before the ACI camera lens assembly and focal plane assembly (FPA) (Figs. 13 and 14), allows a broadband image to be filtered onto the camera $\mathrm{CCD}$, reflecting a green-to-red $(500-600 \mathrm{~nm})$ region of the spectrum.

ACI Description ACI is derived from the MSL MAHLI design (Edgett et al. 2012), but the optics are very different. The optics consist of two sections, the ACI Objective and the ACI Camera Lens Assembly (Figs. 13 and 14). The ACI Objective is common to all three SHERLOC optical paths (Figs. 13 and 14), and focuses the camera and laser on targets that range over working distances (measured from the external window to the target) of 40 to 56 $\mathrm{mm}$. The ACI Objective is mounted to the front end of the STA and the ACI Focal Plane Assembly (FPA) and Camera Head Electronics (CHE) are mounted inside the back chassis of the STA.

The ACI Objective, fronted by a sapphire window, is a telecentric lens composed of four lenses, all of which are uncoated and fabricated from UV-grade fused silica or fluorescencefree magnesium fluoride. The four-element ACI Camera Lens Assembly is made with commercial glasses (fabricated by Optimax) that move as a group on a linear rail to form an image of the target on an On Semiconductor KAI-2020M 1600 x 1200 greyscale CCD sensor with microlenses and no cover glass. The detector is grayscale since the spectral range that is being observed is 500 to $600 \mathrm{~nm}$. This narrow spectral bandpass enabled meeting the required imaging performance.

The ACI Objective is housed within a hardware element that superficially resembles the MSL MAHLI and WATSON lens housing (Figs. 1, 8, and 12). It is nearly identical to the MSL MAHLI design except for the different optics, an opaque dust cover, and an internal AlGaN calibration target for the spectrometer (see Sect. 9.7.2). The internal calibration target is mounted on the inside of the dust cover. The dust cover is therefore opaque and composed of solid aluminum. Like MSL MAHLI and Perseverance's WATSON, ACI Objective lens focus and dust cover are actuated by single Cobham (formerly Aeroflex) $10 \mathrm{~mm}$ stepper motor per the design of DiBiase and Laramee (2009). 
At the other end of the ACI optics, the FPA and CHE are also identical to that of the MSL MAHLI and WATSON design, except for the use of the KAI-2020M (grayscale) instead of KAI-2020CM (color) CCD. ACI control and data storage functions are operated by the SHERLOC Digital Electronics Assembly (DEA), a hardware element (Fig. 24b) located inside the rover chassis. DEA functions and data storage are shared by the ACI and WATSON.

ACI targets can be illuminated by sunlight, or, like MAHLI and WATSON, by two groups of two white light LEDs (Avago Technologies HSMW-10x) or one group of $365 \mathrm{~nm} \mathrm{UV}$ LEDS (Nichia NSHU550B) (Fig. 12). The LEDs, their locations and operability, and the SHERLOC dust cover and focus mechanism, are all from the MSL MAHLI heritage design.

ACI Capabilities Except for the optical and bandpass differences, the MAHLI-based ACI retains many of the basic functions available on the MSL MAHLI (Edgett et al. 2012, 2015) and on Perseverance's WATSON, including full-frame and sub-frame imaging, manual and auto-focus, manual and auto-exposure, video, lossless and lossy onboard image and video compression, focus stack acquisition and onboard focal plane merging, and "thumbnail" image generation. In addition, robotic arm positioning of SHERLOC can be used to acquire ACI image mosaics or stereo pair images. The onboard focus stack capability, provides opportunities to merge 8 images consecutively acquired by stepping through a commanded focus range while the camera is held in place.

ACI capabilities were predominately driven by the ability to do both imaging and spectroscopy and differs notably from WATSON for both focusing, image quality, resolution, field of view, and depth of field (DOF). The focusing, DOF, and telecentric requirements for spectroscopy led to a fixed focal length imager with a nominal focus at $48 \mathrm{~mm}$ with $+/-7$ $\mathrm{mm}$ focusing range. The DOF was driven by the ability to focus the laser on the target within the laser waist for both Raman and fluorescence but still be able to allow for surface relief caused by abraded surfaces of $+/-250$ microns. The as-built ACI met its requirements but as a result of optical offsets between the imager and spectrometer boresights, the spectroscopy system is limited to $15.8 \mathrm{~mm}$ full range focus. Operationally, the ACI mechanism will be offset during operations for optimal imaging and spectroscopy best focus performance. A full frame ACI image covers a $16.16 \mathrm{~mm}$ x $12.12 \mathrm{~mm}$ surface area with an $11.7 \mathrm{~mm}$ diameter non-vignetted field of view with a scale of $10.1 \mu \mathrm{m} /$ pixel over the full range of focus.

In comparison to WATSON, ACI provides high resolution spatial images at a greater working distance and thus provides additional opportunities to obtain high resolution images during nominal operations with less risk to instrument safety. An example of the potential benefits is shown in the simulation in Fig. 22. While the ACI provides high spatial resolution images, its field of view is limited and relies on the wide FOV enabled by WATSON for context. Another example for real-world-comparison is shown in Fig. 52.

\subsubsection{WATSON}

WATSON provides the second of the investigation's two visible wavelength imaging capabilities. WATSON, a copy of the MSL MAHLI (Edgett et al. 2012), is a color camera with a focusable macro lens. Using Perseverance's robotic arm to position the camera, WATSON can focus on subjects at working distances of $\sim 1.8 \mathrm{~cm}$ to infinity with pixel scales ranging from as high as $13.1 \mu \mathrm{m} /$ pixel at $1.8 \mathrm{~cm}$ range to $17.7 \mu \mathrm{m} /$ pixel at $3 \mathrm{~cm}$ range, $31 \mu \mathrm{m} / \mathrm{pixel}$ (comparable to the MER MI cameras) at $6.6 \mathrm{~cm}$ range, $100 \mu \mathrm{m} / \mathrm{pixel}$ at $25.5 \mathrm{~cm}$ range, and even lower spatial resolution at infinity focus. Operationally, based on MSL MAHLI experience (Yingst et al. 2016), the closest range WATSON images will typically be in the 16-18 $\mu \mathrm{m} /$ pixel range, allowing discrimination between silt and very fine sand (Fig. 23a, b). 


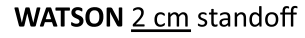

Nominal Operations

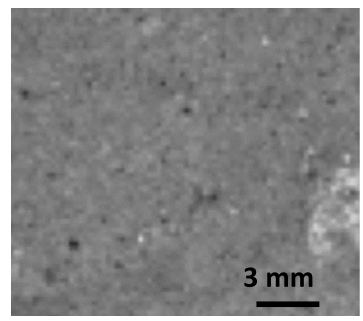

WATSON $1 \mathrm{~cm}$ standoff

Special case operation

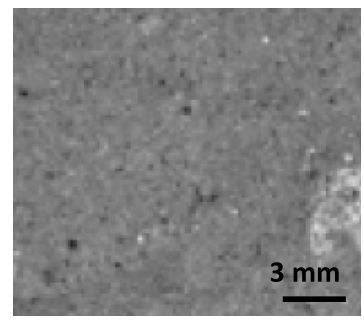

$\mathrm{ACl} \underline{5 \mathrm{~cm}}$ standoff

Nominal Operations

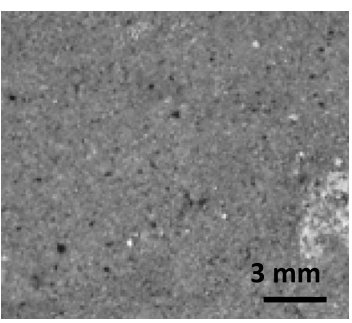

Fig. 22 Images of a fine-grained rock target collected by the engineering model ACI at $5 \mathrm{~cm}$ working distance. The WATSON images are simulated for $2 \mathrm{~cm}$ and $1 \mathrm{~cm}$ working distances

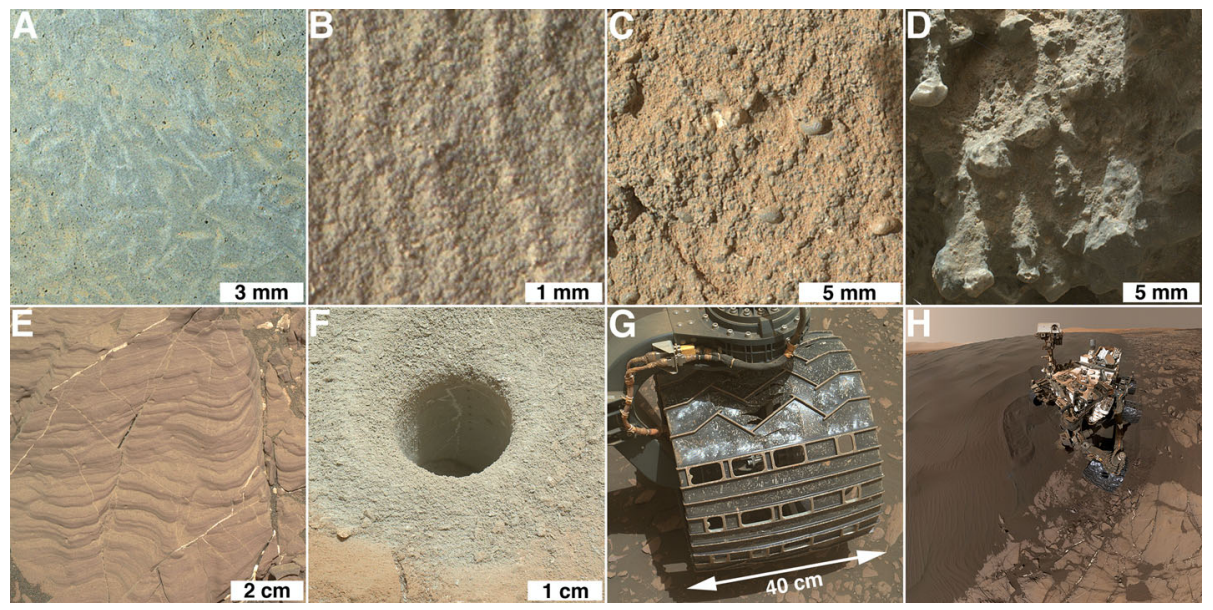

Fig. 23 Example WATSON science and science-enabling support imaging as illustrated by the MSL MAHLI. Please note differences in scale. (A) Mudstone, grains smaller than coarse silt, with gypsum crystal pseudomorphs (Kah et al. 2018); MAHLI night image 809MH0004460020300906C00 illuminated by white light LEDs. (B) Sandstone composed of well-sorted, very fine sand; MAHLI focus merge product 1438MH0001530000503527R00. (C) Gray sandstone with thin coating of orangebrown aeolian dust; MAHLI focus merge product 0999MH0001630000204827R00. (D) Conglomerate; MAHLI focus merge product 1411MH0005840000503008R00. (E) Fine laminae in mudstone outcrop; MAHLI image 1811MH0001900010701304C00. (F) Drill hole wall and cuttings details; MAHLI focus merge product 0270MH0002520000102916R00. (G) Rover hardware inspection (wheel); MAHLI image 2407MH0007710010900396E01. (H) Rover self-portrait for hardware inspection and geological context; MAHLI mosaic acquired Sol 1228

WATSON will obtain Mars science data in the form of close-up (macrophotographic) images of geological materials and science-enabling engineering images. Engineering activities include support for core sample extraction as well as rover and science instrument hardware inspections and diagnostics. Similar activities have been routinely performed on Mars since 2012 using the MSL MAHLI (Fig. 23); Minitti et al. 2013; Robinson et al. 2013; Anderson et al. 2015; Yingst et al. 2016; Abbey et al. 2019). WATSON images of geological targets include provision of context imaging for core samples and SHERLOC and PIXL spectroscopy. Like MAHLI (Minitti et al. 2013; Siebach et al. 2017; VanBommel et al. 2017; Ehlmann et al. 2017; Banham et al. 2018; Kah et al. 2018; Edgett and Newsom 2018; Sun 
Fig. 24 WATSON hardware elements. (a) WATSON camera head. (b) Digital Electronics Assembly (DEA) shared by WATSON and ACI. (c) Multiplexer (MUX) unit shared by WATSON and ACI. The pocketknife is $88.9 \mathrm{~mm}$ long
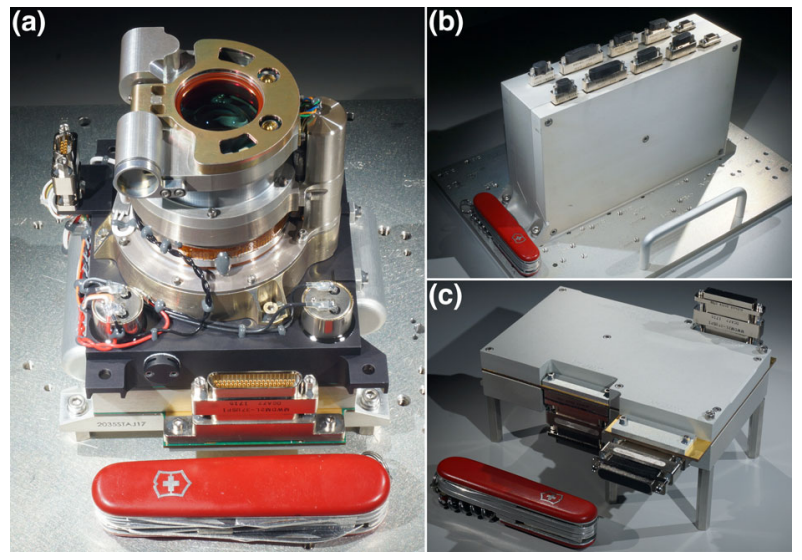

et al. 2019; Rivera-Hernández et al. 2020), WATSON images will provide grain scale information about rock, regolith, and aeolian deposit texture (e.g., grain and crystal size, shape, abundance), structure (laminae, deformation), and assist with the search for biosignatures (e.g., Summons et al. 2011). Also, like MAHLI (Minitti et al. 2014; Fisk et al. 2015), images can be acquired at night using white light and ultraviolet Light Emitting Diode (LED) illuminators.

WATSON Description WATSON consists of one unique hardware element and two shared with the ACI (Fig. 24). Exclusive to WATSON is its camera head, which is a copy of its MSL MAHLI counterpart. The shared elements are DEA and the MUX subsystems (Sect. 5.2.1). The camera head electronics, DEA electronics, and flight software are copies of the MSL MAHLI, Mast Cameras, Mars Descent Imager (Edgett et al. 2012; Malin et al. 2017), and Mars 2020 Mast Camera Zoom (Mastcam-Z; Bell et al. 2020, this journal) instruments. Indeed, both the WATSON camera head electronics printed circuit board (PCB) assembly and the DEA PCB assembly are residual assemblies from the MSL Mastcam, MAHLI and MARDI effort.

The WATSON camera head (Fig. 24a) is part of the STA (Fig. 1). WATSON is not boresighted with the ACI; thus, robotic arm motion is necessary to position the two different subsystems. The camera head consists of an optomechanical assembly (lens) mated to a focal plane and electronics assembly (camera body). The optomechanical assembly includes lens optics; white light and $365 \mathrm{~nm}$ ultraviolet LEDs; an actuated, transparent (Lexan ${ }^{\mathrm{TM}}$ ) dust cover; and a Cobham (formerly Aeroflex) $10 \mathrm{~mm}$ stepper motor for dust cover motion and lens focus. The optics design and details were described by Ghaemi (2009) and Edgett et al. (2012); the mechanism and lens housing were described by DiBiase and Laramee (2009); the LEDs and dust cover window were presented by Edgett et al. (2012). The dust cover is transparent to allow imaging when the cover is closed. On Mars, the cover is likely to become coated by a thin film of dust during the rover's terminal descent to the surface (Edgett et al. 2015), but it is usually possible (and under windy conditions it is sometimes the only good option) to obtain at least a murky image through that dust coating (Edgett et al. 2015). The camera head can be operated on Mars at temperatures between $-40{ }^{\circ} \mathrm{C}$ and $+40{ }^{\circ} \mathrm{C}$. The equivalent MSL MAHLI hardware has been subjected to $>3100$ sols (and counting) of mechanism operations plus diurnal and seasonal temperature variations on Mars. 
Fig. 25 Diagram showing relations between WATSON lens transmission, dust cover Lexan $^{\mathrm{TM}}$ transmission, and system-level red, green, and blue response (quantum efficiency, $\mathrm{QE}$, times transmittance, $\mathrm{T}$ ) as a function of wavelength based on lens, CCD, and dust cover equivalence to MSL MAHLI

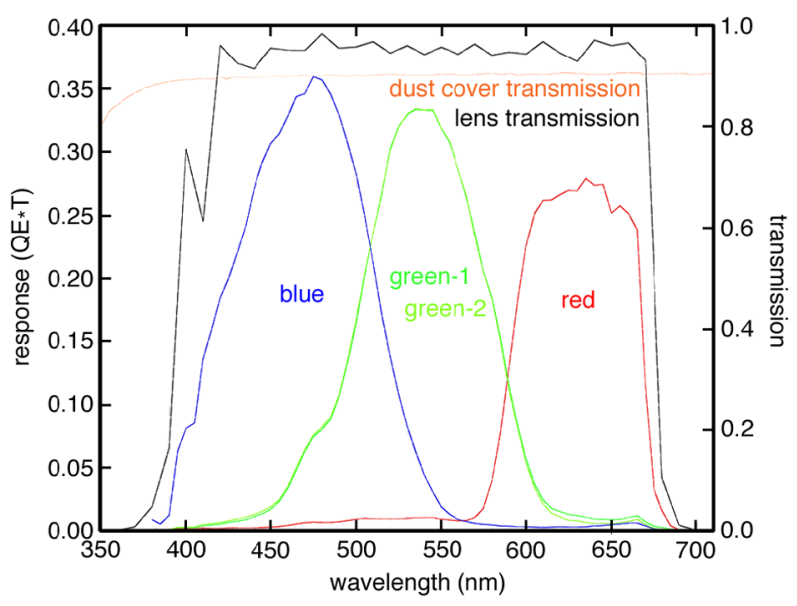

The focal plane assembly is designed around a ON Semiconductor KAI-2020CM interline transfer CCD that provides Bayer pattern (Bayer 1976) RGB (red, green, blue) color imaging on an array of 1600 by 1200 photoactive pixels of $7.4 \mu \mathrm{m}$ by $7.4 \mu \mathrm{m}$ size (Edgett et al. 2012; Malin et al. 2017). Undesired near-infrared radiation is blocked by a coating on the inside surface of WATSON's front lens element, a sapphire window. The combination of lens transmission properties, the infrared cut-off filter, and the RGB microfilters on the CCD, provide spectral throughput over the 395 to $670 \mathrm{~nm}$ range as shown in (Fig. 25).

WATSON Capabilities WATSON retains all of the functions and capabilities of the MAHLI, including full-frame and sub-frame imaging, manual and auto-focus, manual and auto-exposure, video, lossless and lossy onboard image and video compression, focus stack acquisition and onboard focal plane merging, onboard Bayer pattern color interpolation, and "thumbnail" image generation (Edgett et al. 2012, 2015). In addition, robotic arm positioning of the camera head is used to acquire image mosaics, nested image suites of increasing spatial resolution, stereo pair images, and multi-position images for Structure from Motion (SfM) quantitative surface relief products (Edgett et al. 2012; Minitti et al. 2013; Yingst et al. 2016; Garvin et al. 2017).

As with MAHLI, the onboard focus stack capability can merge 8 images consecutively acquired by stepping through a commanded focus range while the camera is held in place (Edgett et al. 2012, 2015). This capability is routinely used on MSL and provides an added form of onboard data compression that converts up to 8 images into two products that are downlinked, a best focus image product and a grayscale range map; an example from WATSON is shown in Fig. 26.

\section{SHERLOC Calibration Target (SCT)}

The SCT (Fig. 27) was designed and constructed at NASA's Johnson Space Center (JSC) with Jacobs Engineering Inc. It is affixed to the front of the rover and includes targets to calibrate the instrument on the surface of Mars. Three targets are specifically heated during cruise to avoid outgassing contamination build up on their surfaces. 


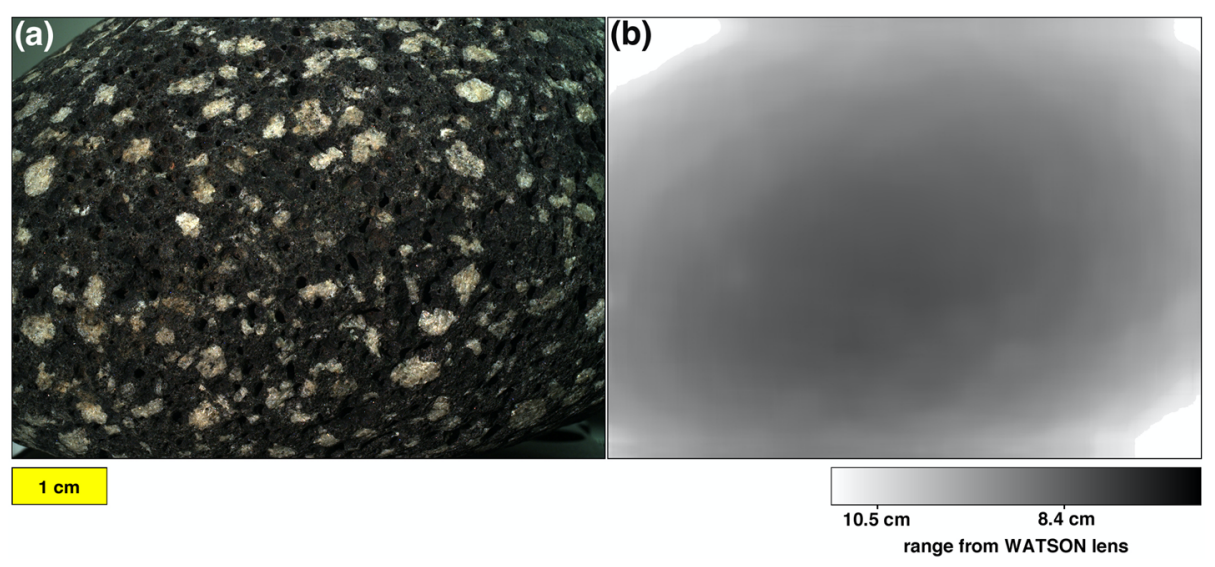

Fig. 26 Example flight unit WATSON focus merge products (a) color, best focus image and (b) grayscale range map produced from 8 focus stack images of a rounded basalt porphyry cobble acquired during clean room ground testing on 07 October 2019. The range map can be used as a rudimentary surface relief map at millimeter scale

Fig. 27 Photograph of the SHERLOC Calibration Target (FM). Numbers are referred to in the text and not printed on the actual target

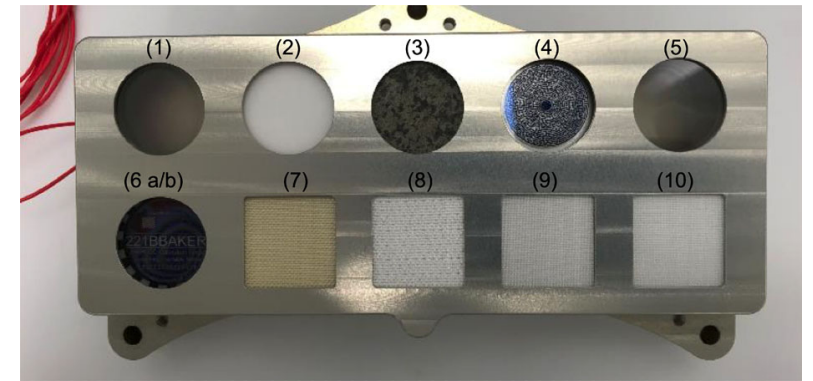

\subsection{SCT Design}

The SCT is $150 \times 89 \times 33 \mathrm{~mm}$ and has a mass of $\sim 437$ grams. Three mounting locations interface with the extension bracket placing the assembly forward-facing on the front of the rover. This configuration allows the STA, through discrete rover arm movements, to access and analyze the each of the SCT targets. The SCT has ten individual targets locations (numbered in Fig. 27); six accommodate $25 \mathrm{~mm}$ round (20 mm exposed) hard targets of various thickness, and four $20 \times 20 \mathrm{~mm}$ square openings which expose a portion of space suit material retained by a soft goods sub-assembly (Fig. 27). A small thermal sub-assembly allows heating of three targets to reduce contamination risk. The design uses compressed wave springs to hold individual targets and sub-assemblies in place. A cover plate protects the targets in ATLO and was removed before flight.

Two targets (number 4 and 6 in Fig. 27) have markings on them specifically designed to calibrate the spatial resolution of both ACI imaging and spectroscopy. Target 4 is a "maze" with chrome coated and uncoated parts that are $200 \mu \mathrm{m}$ thick. The laser is focused to a $100 \mu \mathrm{m}$ in diameter spot at optimum focus. By scanning the laser across the maze in Target 4 and monitoring the intensity of the silica signal, we guarantee the laser hits both chrome and silica through a pre-programmed line scan - regardless of arm placement with respect to distance to target, angle to target, and rotation of the turret. This will permit to calibration of 
the intensity and size of the laser spot, the response of the SCCD and the pointing accuracy of the scanning mirror without requiring ground-in-the-loop operations. The maze was designed to return results regardless of the placement accuracy of the arm over all temperature ranges.

The SHERLOC Geocache target (Target 6b in Fig. 25; shown in detail in Fig. 26c) was designed to monitor spatial resolution performance as a function of focus position for the WATSON and ACI cameras. The top of the calibration target includes a resolution graphic developed from the 1951 USAF Resolution Test chart that includes line pairs ranging from 4 line pairs per millimeter $(\mathrm{mm})$ to 114 lines pairs per $\mathrm{mm}$. This target will enable the team to demonstrate resolution across the range of motion of the optics for the WATSON color imager, and the autofocusing capability of the ACI. Rather than repeat the paired lines resolution target present on the upper portion of that target, the team chose to add in a graphical representation that could be used to determine resolution. Like the maze target, the Dancing Men Cypher allows the team to identify resolution without the need for groundin-the-loop activities such as re-positioning the robotic arm.

\subsection{SCT Target Materials}

The top target row consists of five targets used for SHERLOC calibration including Raman and fluorescence spectral accuracy, response curve, ambient light reflection, spectral mapping, and laser parameters. The bottom target row consists of a selected suite of space suit materials, which also function as secondary calibration targets. Table 4 lists the target functions/materials (numbers are shown in Fig. 27):

Three targets additionally serve unique education and public outreach purposes. The Mars meteorite, a slice of Sayh al Uhaymir 008 ( $\mathrm{SaU}$ 008) collected in Oman in 1999, will be the first piece of Mars to be returned to the planet's surface (Fig. 28a). The intensity target design incorporates an intricate maze (Fig. 28b), and the clear polycarbonate space suit visor material is backed by a distinctive Geocaching trackable marker (Fig. 28c see e.g., Cameron 2017).

\subsection{SCT Cleaning and Assembly}

To achieve Mars 2020 and SHERLOC cleaning and contamination control standards, we used the NASA Johnson Space Center (JSC) Astromaterials Research and Exploration Science Division (ARES) curation cleanroom facilities, expertise, and best practices. All SCT metallic components, hard targets, soft goods targets, and handling tools were cleaned and verified to meet or exceed established requirements. Assembly occurred in a dedicated ISO Class 5 laboratory that was monitored for particulates, as well as inorganic and organic airborne molecular contamination.

Three SCTs were assembled, the Engineering Model (EM), the Flight Model (FM), and the Flight Spare (FS). The EM was used for assembly fit-checks and as a mass simulator for dynamics testing and in rover level System Thermal Tests (STT). A fourth SCT will be assembled as a Curation Model (CM), following the same cleaning and assembly protocols, and will be stored at JSC with other Mars 2020 components for future analysis and referral. Only the FM and FS include the Mars meteorite target and the main difference between them is that the FS meteorite sample has a small $1 \mathrm{~mm}$ vug off the center of the target. The EM and CM include a terrestrial basalt in that target location. As a result of a contamination event that impacted the FM during ATLO, the FS replaced the FM and the FS is the target on the rover. 
Table 4 SCT targets and their primary and secondary function

SHERLOC Calibration Target Primary function $\quad$ Secondary function

(1) AlGaN on Sapphire (262 nm)

(2) Diffusil Diffuser

(3) Mars Meteorite (SaU 008)

(4) Intensity target (Maze/fused silica)

End to End instrument performance

(5) AlGaN on Sapphire (335 nm)

Raman calibration
Ambient light calibration
Fluorescence calibration

End to End instrument performance Alteration in Mars environment during mission

Image/Spectroscopy

Mapping calibration

HEOMD and Education/Public Outreach

(6a) Polycarbonate Spacesuit Visor

Assess changes in material spectral properties/textures

(6b) Geocache coin (opal glass)

Resolution target

Education/Public Outreach

Soft Goods (HEOMD)

(7) Vectran

(8) Orthofabric

(9) Teflon

(10) nGimat coated Teflon
Assess changes in material spectral properties/textures

Assess changes in material spectral properties/textures

Assess changes in material spectral Raman spectral calibration properties/textures

Assess changes in material spectral properties/textures

Fig. 28 Photograph of SHERLOC Calibration Targets (from the FM). (a) \#3 Mars Meteorite, (b) \#4 Intensity Target Maze, and (c) \#6b Geocaching Trackable Marker coin

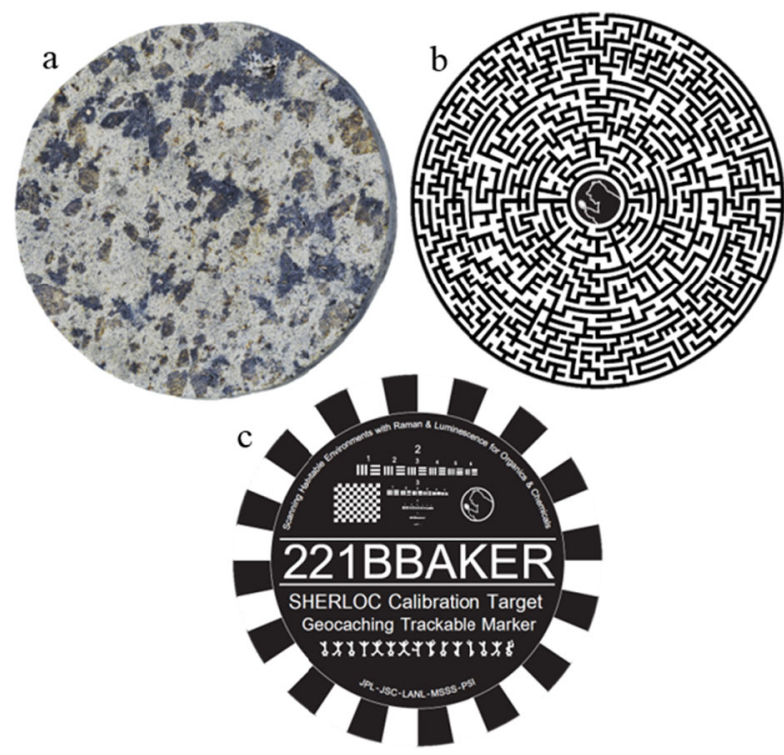




\subsection{SCT Testing and Validation}

The FM and FS completed both random vibration and shock testing in each of the three component axes at JSC's Energy Systems Test Area. Additionally, the FM and FS successfully completed thermal function testing, high-resolution imaging and optical inspection, and bake out requirements. Thermal vacuum testing occurred with the rover at JPL.

\section{Thermal Subsystem}

All of the individual components of the SHERLOC instrument are designed to operate in the Martian environment at the Jezero field site for the duration of the Perseverance mission. Worst case hot and cold environments were provided by the Mars 2020 Project to develop the thermal systems architecture to ensure instrument operability and survivability. Worst case cold is defined as the coldest the instrument will experience right before dawn in winter at the Jezero landing site. Worst case warm is defined as the warmest the instrument will experience which is during cruise. There are four basic functional requirements on the thermal system for SHERLOC:

1. Survive the worst case cold conditions on Mars with minimal the survival energy.

2. Warm-up the instrument prior to commencement of instrument start-up for a science investigation.

3. Maintain the Spectrometer Charged Coupled Device (SCCD) below $-20{ }^{\circ} \mathrm{C}$ during acquisition of spectrometer spectral maps.

4. Provide decontamination heating for the instrument chassis and the calibration target to ensure contamination materials do not collect on sensitive surfaces during spacecraft cruise to Mars.

The SHERLOC instrument contains several major components that are affected by the thermal system and its various functions. The thermal sub-systems include: survival heating, warm-up heating, decontamination heating, phase change material heat sink, and the rover heat rejection system. These thermal regions are shown in Table 5 which also describes the control type and the temperature range.

\subsection{Survival Heating System}

Most of the SHERLOC instrument components are capable of surviving down to $-90{ }^{\circ} \mathrm{C}$ without requiring a survival heater. The LPS and the SDE are two exceptions. The LPS minimum temperature is limited to $-80^{\circ} \mathrm{C}$ and the SDE is limited to $-40{ }^{\circ} \mathrm{C}$. Survival heaters and mechanical thermostats, shown in Fig. 29, are placed on these components with minimum set points that are $5{ }^{\circ} \mathrm{C}$ above their allowable temperature limits. Two thermostats are placed in series with each other on each heater circuit in accordance with standard JPL practice for reducing the risk of thermostat failure when activated. Under the worst case cold conditions on Mars, the maximum predicted survival energy is about 110 Watt-hours per sol. The SDE has a single layer aluminized Kapton blanket with a $5 \mathrm{~mm}$ gap between the blanket and the SDE chassis to reduce heat loss to the environment. The cover blanket is not shown in Fig. 29. The SDE minimum allowable temperature is about $60{ }^{\circ} \mathrm{C}$ warmer than the coldest ambient temperature on Mars, thus the blanket is necessary to meet the survival energy budget allocated to SHERLOC. The SDE dissipates less than 1 Watt when operating, which is less than the survival heater power of about 4 Watts, so the SDE easily stays within its 
Table 5 SHERLOC thermal subsystem overview

SHERLOC Thermal Subsystems

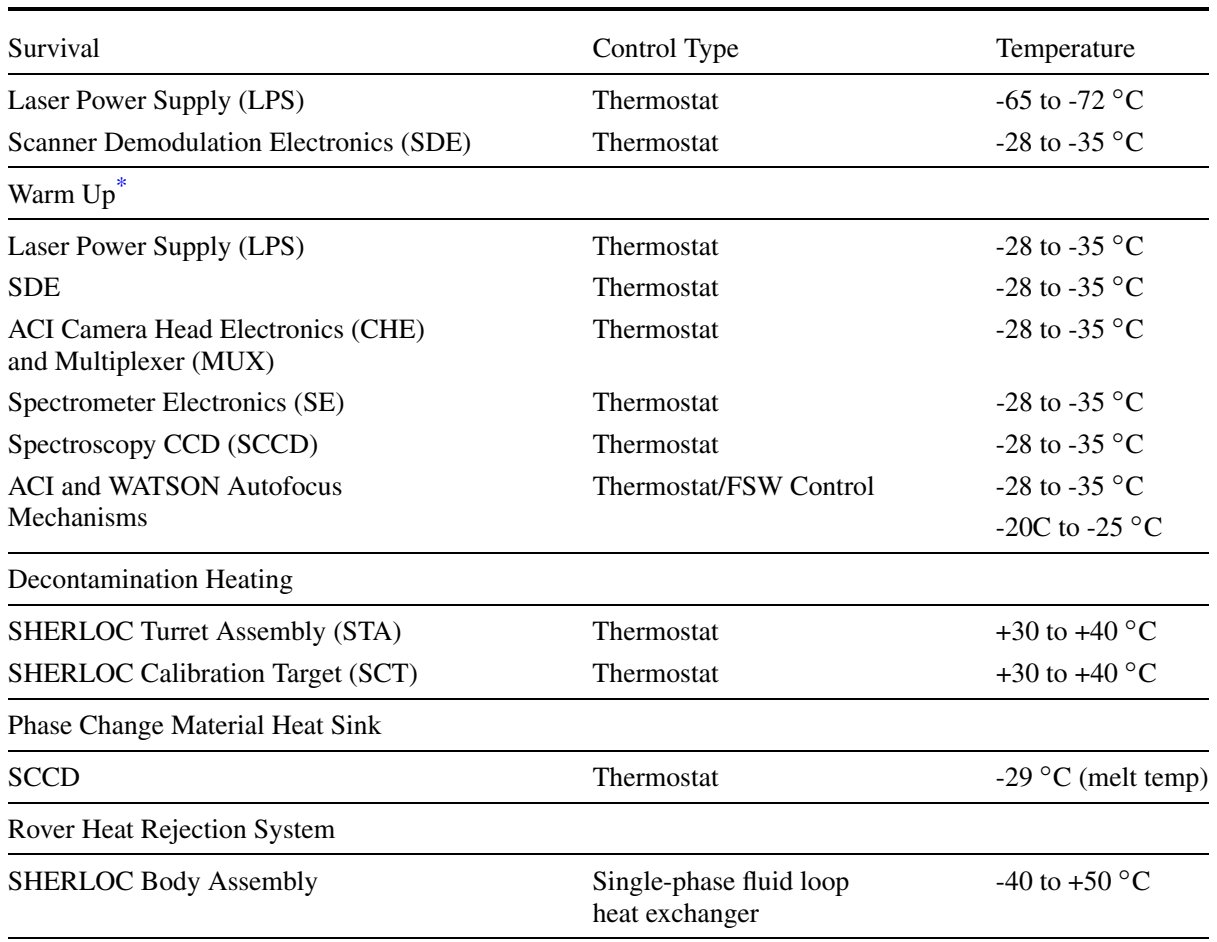

*Warm-up zone is controlled by rover flight software thermal module on the WATSON and ACI mechanisms above $-25^{\circ} \mathrm{C}$ to minimize noise impact of thermostatic switching during operations.

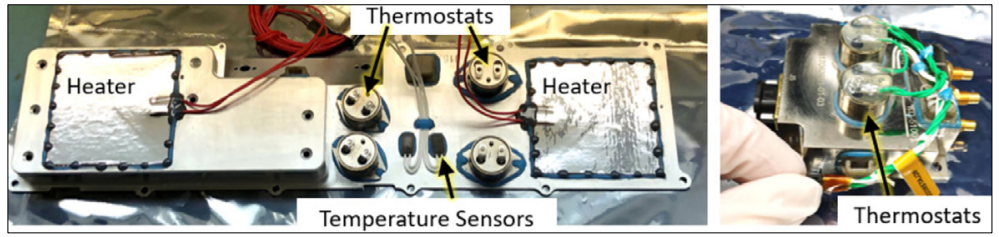

Fig. 29 Survival heating circuit hardware for the LPS (right) and the SDE (left)

upper temperature limit of $40{ }^{\circ} \mathrm{C}$ when operating. The LPS dissipates up to 15 Watts of heat when operating, thus it cannot be blanketed like the SDE to minimize heat loss when not operating. Since the LPS minimum temperature is only about $20^{\circ} \mathrm{C}$ warmer than the coldest expected ambient temperature, the survival heat loss is acceptable under the worst-case cold conditions.

\subsection{Warm-up Heating System}

Many of the electronics and mechanisms on SHERLOC have a minimum operating temperature of $-40{ }^{\circ} \mathrm{C}$ and require warm-up prior to start-up. The warm-up heating circuit uses 


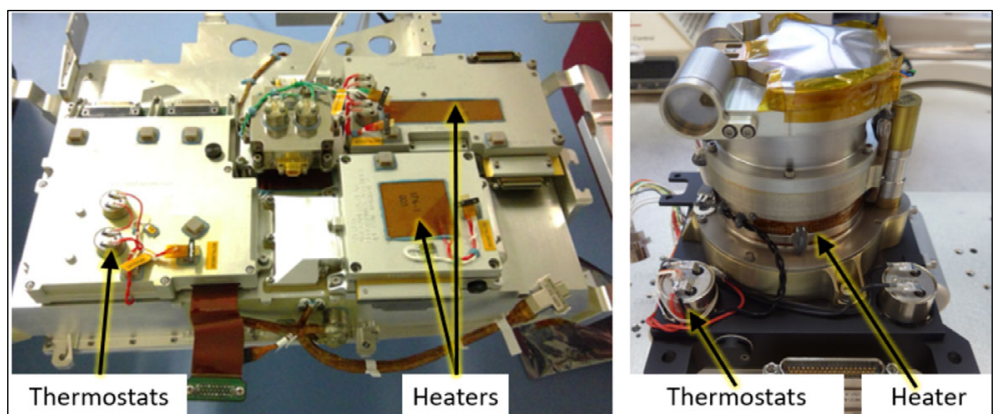

Fig. 30 Warm-up heating circuit hardware for the SHERLOC instrument. Left image shows SE, MUX, and CHE. Right image shows the WATSON camera head

redundant mechanical thermostats with a set point of $-35{ }^{\circ} \mathrm{C}$ for the externally mounted components to regulate temperature above the minimum allowable operating temperatures. Inside the STA, the warm-up heater thermostats for the SCCD have a set point of $-42{ }^{\circ} \mathrm{C}$. The lower set point for the SCCD is provides as much operating temperature margin on the SCCD as possible. The signal to noise ratio on the SCCD meets Raman spectroscopy data requirements when the SCCD temperature is less than $-20{ }^{\circ} \mathrm{C}$ during spectroscopic acquisition.

Each of the electronics boxes on the instrument have their own heater and thermostats as shown in Fig. 30. However, there are a few cases where thermostats control heaters on multiple components. The thermostats on the Multiplexer controls the heater on the CHE and the thermostats on the WATSON camera head control the heaters on the autofocus mechanisms. The warm-up heaters are all on one circuit which is controlled by the rover flight software which monitors the electronics component temperatures.

\subsection{Decontamination Heating System}

The SHERLOC instrument is designed to detect potential organic materials and is highly sensitive to contamination from organic molecules which have many sources on the rover. A set of heaters are distributed over the STA, as shown in Fig. 31, to keep it warm relative to surrounding surfaces while stowed on the rover turret during the interplanetary cruise to Mars. The decontamination heaters operate from just before launch to just before entry into the Martian atmosphere, except for up to 60 minutes when the cruise vehicle is in eclipse right after launch. They are designed to operate during cruise to avoid contamination from outgassing components on the rover. These are not intended for use in the Mars atmosphere. The SCT, which is mounted to the front of the rover chassis, has its own heater to keep three specific targets warmer than its surrounding surfaces (shown inside the red box, right side of Fig. 31). The decontamination heaters are controlled by flight software but have mechanical thermostats in place for overtemperature protection.

\subsection{Phase Change Material (PCM) Heat Sink}

The SCCD dissipates about 1 Watt when operating and is expected to operate for up to 1 hour for a nominal operation. The PCM heat sink was designed and built to keep the SCCD temperature below $-20^{\circ} \mathrm{C}$ during Raman spectroscopy operations. The SCCD assembly has 2 PCM units installed as shown in Fig. 32. This assembly is thermally isolated from the rest 


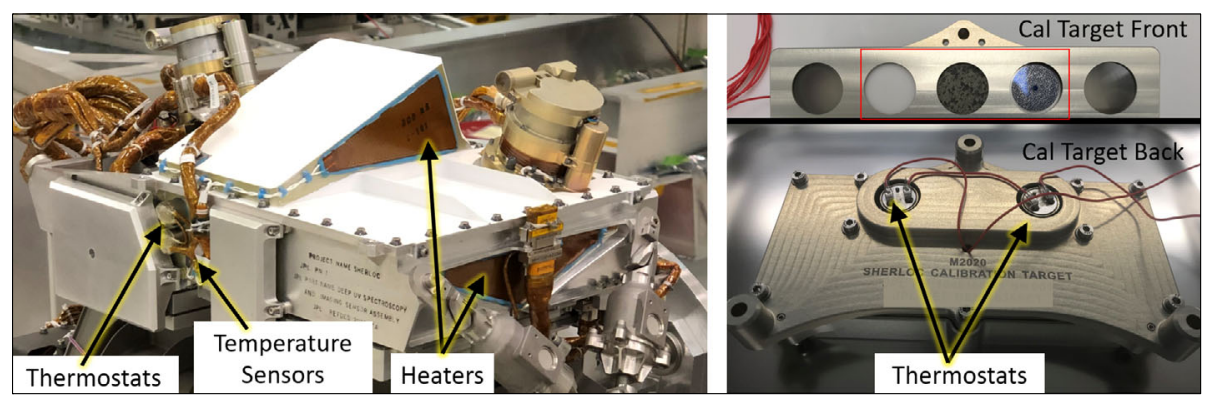

Fig. 31 Decontamination heating circuit hardware for the SHERLOC instrument. The SCT shown is it the FM target

Fig. 32 Phase Change Material (PCM) heat sinks attached to the SCCD mounting interface

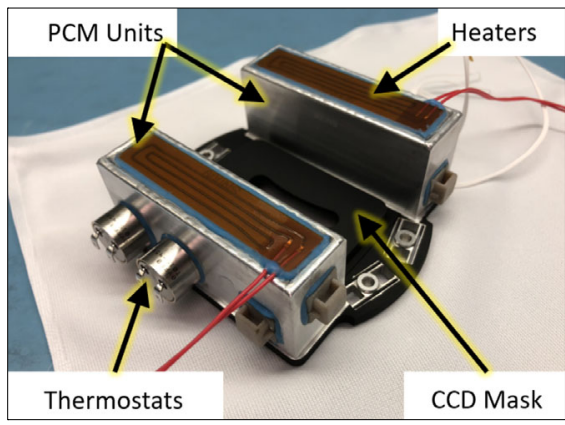

of the instrument such that, besides power dissipation from the SCCD, the major loss/gain of heat comes from radiative transfer. The PCM units have an aluminum enclosure with a carbon fiber matrix core with high thermal conductivity that is filled with n-decane. This alkane material has a melting point of $-29^{\circ} \mathrm{C}$. The energy absorbed by each PCM unit is approximately $3.3 \mathrm{~kJ}$ over the temperature range of -30 to $-20{ }^{\circ} \mathrm{C}$. The PCM units refreeze overnight if they melt during instrument operations and can be used on a daily basis. The aluminum housings are welded enclosures to ensure the n-decane will not leak because it would become a significant organic contaminant for the instrument. The units were filled at $+80{ }^{\circ} \mathrm{C}$ which provides $30{ }^{\circ} \mathrm{C}$ margin of the maximum allowable operating temperature of $+50{ }^{\circ} \mathrm{C}$. Although the units have an internal pressure less than Earth ambient pressure, they are slightly pressurized in the Mars environment or in vacuum during cruise to Mars. The warm-up heaters and thermostats for the SCCD are attached to the PCM housings. The warm-up heater thermostats have a set point cold enough to prevent melting of the PCM while warming up the SCCD to its minimum operating temperature limit.

\subsection{Rover Heat Rejection System}

The SHERLOC instrument has an electronics box (SHERLOC Body Assembly, SBA; Fig. 9) that is mounted to the Rover Avionics Mounting Plate (RAMP). The RAMP serves as the mechanical and thermal interface for all instrument electronics and rover avionics. The SBA dissipates up to 55 Watts to the RAMP where the heat is absorbed by an embedded single-phase fluid loop heat exchanger which circulates fluid from the electronic chasses to radiator surfaces outside the rover chassis. The RAMP is capable of maintaining the SBA 
Fig. 33 TRIOPTICS

OptiCentric lens-adjustment instrument demonstrating placement of optics into structural hardware mount

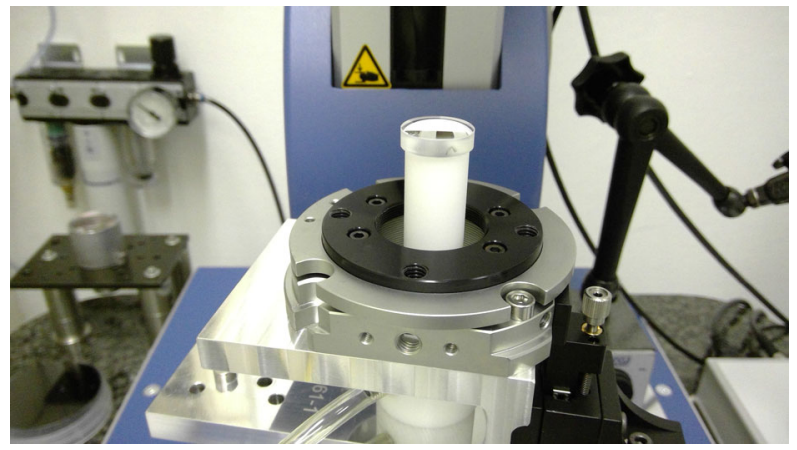

within its allowable operating temperature range of -40 to $+50{ }^{\circ} \mathrm{C}$ under all conditions during the mission. There are no thermal hardware items installed on the SBA other than temperature sensors on the power distribution unit board and the digital electronics assembly board inside the chassis.

\section{SHERLOC Integration and Component Testing}

\subsection{SHERLOC Instrument Optical Alignment}

Because of the sensitive contamination constraints associated with building an ultraviolet Raman and fluorescence spectrometer, all optical assembly and alignment operations were conducted in an ISO 7 cleanroom, often on an ISO 5 flowbench. All optics were stored in Teflon cases and handled by Teflon tools and nitrile gloves. Both transmissive lens assemblies were aligned using a TRIOPTICS OptiCentric lens-alignment instrument by holding each lens element on a Teflon vacuum pedestal, as shown in Fig. 33. These pedestals were attached to stages to articulate the optics in five degrees of freedom within their lens housings. All flat reflective optics were aligned parallel to their mount's mechanical pads using the autocollimator of the OptiCentric instrument. They were then mounted to the optical bench structure that was fabricated to tight tolerances, the complete assembly of which is shown in Fig. 34.

SHERLOC Spectrometer optics were aligned as two independent modules: The spectrometer relay, that injects the collected fluorescence emissions and Raman scattered light into the spectrometer, was aligned with collimated input from an interferometer to collimated output that was reflected off a reference mirror to create a double-pass test of the two OAP mirror module. Both OAP mirrors were held by vacuum as they were articulated to achieve output collimation with minimum aberration. The spectrometer slit was then aligned to the internal focus of this OAP mirror pair using a Foucault technique, guided by the double-pass interferogram.

The sphere-asphere spectrometer optics were aligned using a visible laser while articulating the Spectrometer's spherical mirror. The point spread functions (PSFs) of various laser spots were assessed across the focal plane of the spectrometer optics using a high-resolution beam-profiling camera. The irradiance distributions of the measured PSFs were compared to their modeled sizes and shapes to achieve alignment.

The SCCD sensor and the fore optics bench were then integrated with the Spectrometer optics to complete the internal SHERLOC optical assembly (Fig. 35). The alignment 
Fig. 34 The foreoptics of the SHERLOC optical bench

Fig. 35 SHERLOC optical bench assembly testing with standard sources
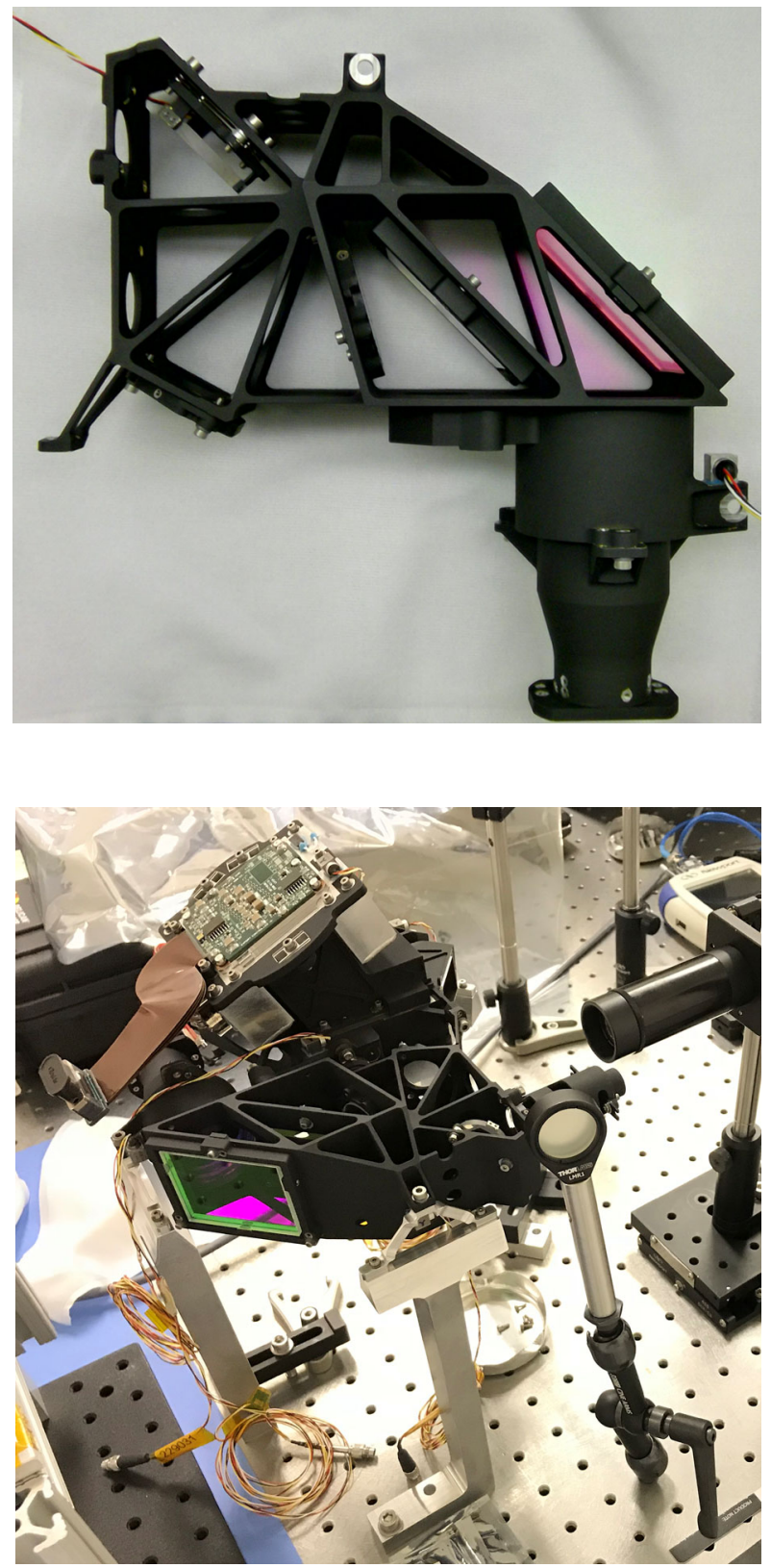

light source was changed to a collimated beam, output from an optical fiber. Into this fiber, mercury and xenon plasma-discharge spectra were input, to cast mercury lines across the Raman region of the SCCD and the broadband xenon continuum across the fluorescence region of the SCCD. These spectral features were focused on and registered laterally to the SCCD pixels by articulating the SCCD itself before it was shimmed and fastened to the spectrometer structure. The hardware configuration for this alignment is shown in Fig. 35. 
The final alignment challenge was to co-align the boresights of all three optical paths by articulating the laser so that its focus is centered within the image of the spectrometer's slit in the instrument's target plane, while keeping both of these spots well centered on the ACI's Focal Plane Array (FPA). This is a three-dimensional alignment problem because all paths must not only be pointed along the same direction, but they also must have collocated focal points, within the performance margin. During this complex alignment procedure, the spectrometer path is held as "truth" to which the other paths are aligned. Alignment of the ACI's FPA was achieved by physically shimming the FPA in its focal plane. A reference to the optimal spectrometer target-space focal coordinate was located in the target plane, and through-focus images of this reference were acquired via the ACI mechanism. The offset between the context image with the sharpest focus and the nominal position of the ACI mechanism was measured, and the corresponding ACI FPA shim thickness change was calculated and installed to focus the Context Imager in the same target plane as the spectrometer. Although it was possible to transversely move the ACI FPA to ensure its lateral alignment, this was not required due to the tight tolerance of the optomechanical components along with the precise alignment of the reflective optics within the SHERLOC optical bench.

The SHERLOC laser optics were initially aligned to expand and collimate the laser using a flight-like laser with similar beam properties; a 35-mm beam-profiling camera measured the propagating beam to assess magnification and collimation as the one lens of the expander was de-spaced and decentered with respect to the other. This alignment provided a starting point for the focus alignment of the laser's focus to the spectrometer in the target plane. To achieve final alignment, the laser was fired, and its target-plane focus location was compared to that of the Spectrometer. The three-dimensional offset between optimal spectrometer focus and optimal laser focus thereby provided the required motion vectors of the laser. Laser focus alignment was achieved by translating one lens of the laser's beam expander with respect to the other. Laser lateral alignment was achieved by physically shimming the laser on its mount pads, slightly tilting the beam through the optical system. This proved to be a tedious but deterministic procedure that converged to achieve better than $30 \mu \mathrm{m}$ lateral overlap and $700 \mu \mathrm{m}$ focal overlap of the laser's caustic within the spectrometer's depth of focus in the target plane. Laser centration was also assessed in the near field via large-area beamprofiling camera; this ensured that the laser output was well centered within the instrument's aperture stop.

\subsection{Deep UV Laser Testing}

Given that the DUV laser is the first of its kind on a space flight instrument, we expended a significant effort to test the laser and characterize its behavior over the Mars thermal range and through vibration (launch, cruise, landing and coring/abrading) conditions. The following sections describe the thermal tests that were performed as well as the key attributes that were analyzed.

\subsubsection{Thermal Vacuum Chamber}

The thermal vacuum chamber is capable of delivering environment temperatures between $+90{ }^{\circ} \mathrm{C}$ and $-150^{\circ} \mathrm{C}$ via electric heaters and a SunPower CryoTel GT cryocooler (Fig. 36).

The laser and laser power supply were bolted into a mechanical cradle that was designed based on the flight mounting hardware. This mechanical mounting assembly was affixed to the environmental chamber for mechanical stability and thermal conductivity, allowing for the test article temperature to be controlled via the environmental chamber thermal shroud. 

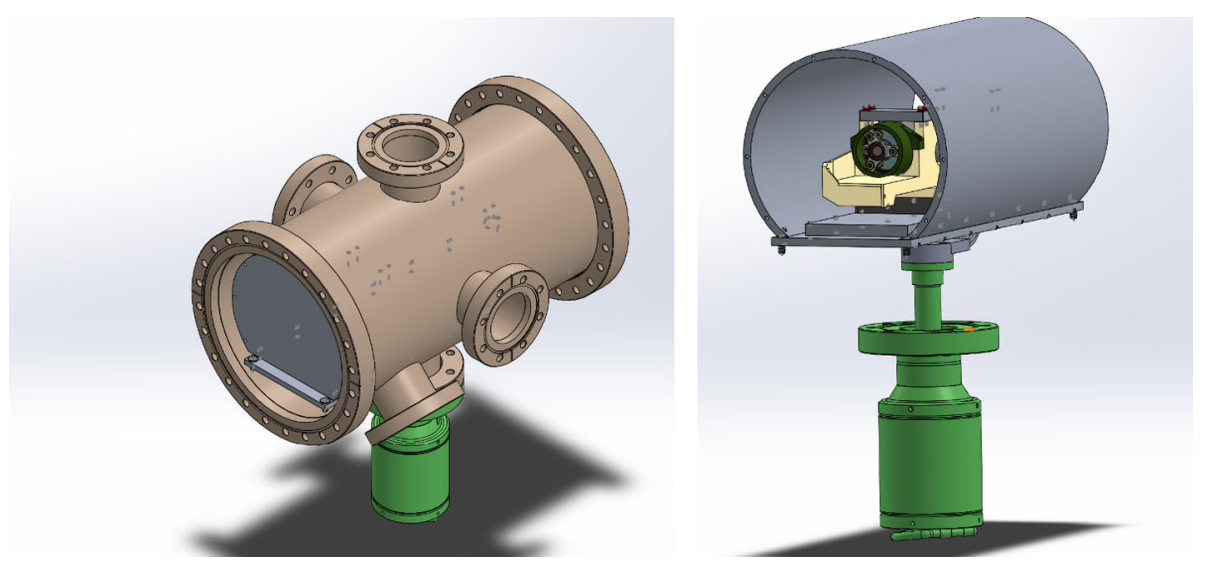

Fig. 36 Overview renderings of the thermal vacuum chamber specifically designed for SHERLOC laser testing. Left: vacuum chamber (brown) with cryocooler (green) mounted on bottom. Right: laser (dark green) mounted in mechanical hardware (beige), affixed to shroud (gray) that is thermally controlled by cryocooler (green)

\subsubsection{Laser Power Supply Operation}

The laser power supply and its corresponding control electronics provide current-regulated output power to the laser, with programmable current, pulse width, repetition rate, and pulse grouping. Typical laser operation requires a $40 \mu$ s pulse of $25 \mathrm{~A}$, with a repetition rate of 80 $\mathrm{Hz}$, and a pulse grouping of typically 165 pulses. To simulate the science operations in the SHERLOC instrument, the operations timing was hardware-controlled using a gating signal within the control electronics. This ensured that the number of pulses and the subsequent delay were well-known. The facility operations software is capable of versatile programmable laser operation to meet the needs of any desired test pattern to emulate SHERLOC instrument science operation.

Temperature A series of E-type thermocouples were affixed to various points on the laser, LPS, mounting hardware, and the environmental chamber thermal shroud. In addition, various $100 \mathrm{ohm}$ PRT sensors were used throughout the environmental chamber, and 1000 ohm PRTs were used on the LPS. Typically, the E-type thermocouples are accurate within roughly $3{ }^{\circ} \mathrm{C}$, while PRTs have accuracies of $0.25 \%$ or better. All temperature monitoring was calibrated and demonstrated to work within the uncertainty of the thermocouples, which was satisfactory for monitoring of temperatures.

Laser Metrology The laser was installed in the test chamber, with laser output directed through a UV-grade fused silica viewport window ( $~ 90 \%$ transmission at $247 \mathrm{~nm}$ ). From there, the laser beam was directed to an optical table with a variety of beam splitters, fold mirrors, and sensors to perform quantitative measurement and analysis of the laser performance when under environmental test conditions (Fig. 37).

\subsubsection{Laser Pulse Characterization}

The output energy of the laser was measured using an Ophir PD-10-C-L-SH sensor head and Juno laser power meter. During operation, the power meter was set to acquire data in 


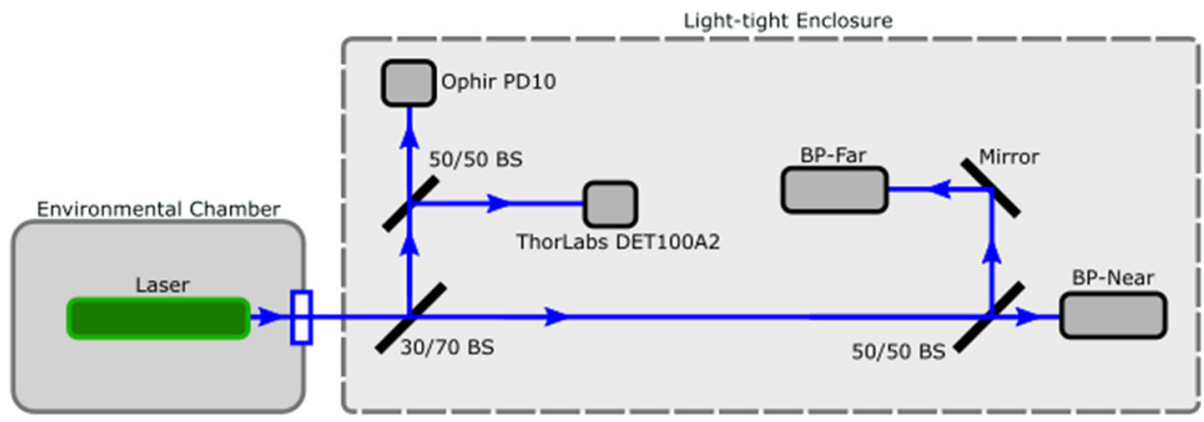

Fig. 37 Schematic of laser metrology. Laser light passes out of environmental chamber via a fused silica viewport and is projected onto an Ophir PD10, Thorlabs DET100A2, and two beam profiler assemblies

threshold triggered mode, and data were streamed/buffered to the facility operations software, where every detected laser pulse was captured. In addition to recording the energy of every detected pulse, the power meter recorded the time of detection with millisecond accuracy. This served as a timestamp event for pulse detection, and provided the most accurate method in the facility for timekeeping during laser operations. All other pulse-by-pulse time-sensitive detection metrology was synchronized to this timestamp.

To account for energy losses due to transmissions and reflections, a radiometry scale factor was determined prior to testing operations. First, the pulse energy of an unobstructed laser was measured at a $45 \mathrm{~cm}$ distance. The pulse energy was then measured with the detector installed at its normal location within the metrology bench, with the vacuum viewport installed. The ratio between these two measurements gave a scale factor that was used to determine the actual energy output from the SHERLOC laser during environmental test conditions.

To measure the time-variation of the laser pulse energy, a ThorLabs DET100A2 biased silicon detector was used. The analog output signal from this biased detector was observed on an oscilloscope and recorded using a NI-6366 analog input at 2 megasamples/s, with an acquisition start hardware-triggered to the laser command pulse output from the NI-9401.

To image the spatial profile of the beam, the laser was directed onto a beam profiler assembly that consists of a BK7 glass microscope slide, focusing optics, and a Basler acA2040-25gm 4-megapixel 8-bit monochromatic camera. The BK7 glass slide downconverted the UV laser light and fluoresces in the visible, which was imaged onto the sensor of the Basler camera. Acquisition start was hardware-triggered using the laser command pulse output from the NI-9401.

The laser output is an annulus spatial profile. Drawing a line along the diameter of the beam, the intensity profile resembled two Gaussian peaks with effectively zero intensity in the middle. The laser beam profile was ideally circular, although some elliptical shape tends to be present. Two of these beam profile acquisition systems were used to provide the minimum information required to measure the spatial property of the laser beam including the pointing $(\mathrm{P})$ direction, the decentration from the mechanical axis $(\mathrm{C})$, and beam expansion properties $\left(\mathrm{M}^{2}\right)$.

To further diagnose operation, the current and voltage delivered from the LPS to the laser was monitored using a Tektronix TCP312A current probe and Tektronix P5200A high voltage differential probe. The analog voltage outputs of these probes were observed on an oscilloscope and recorded using additional input channels of the NI-6366. In this optional 


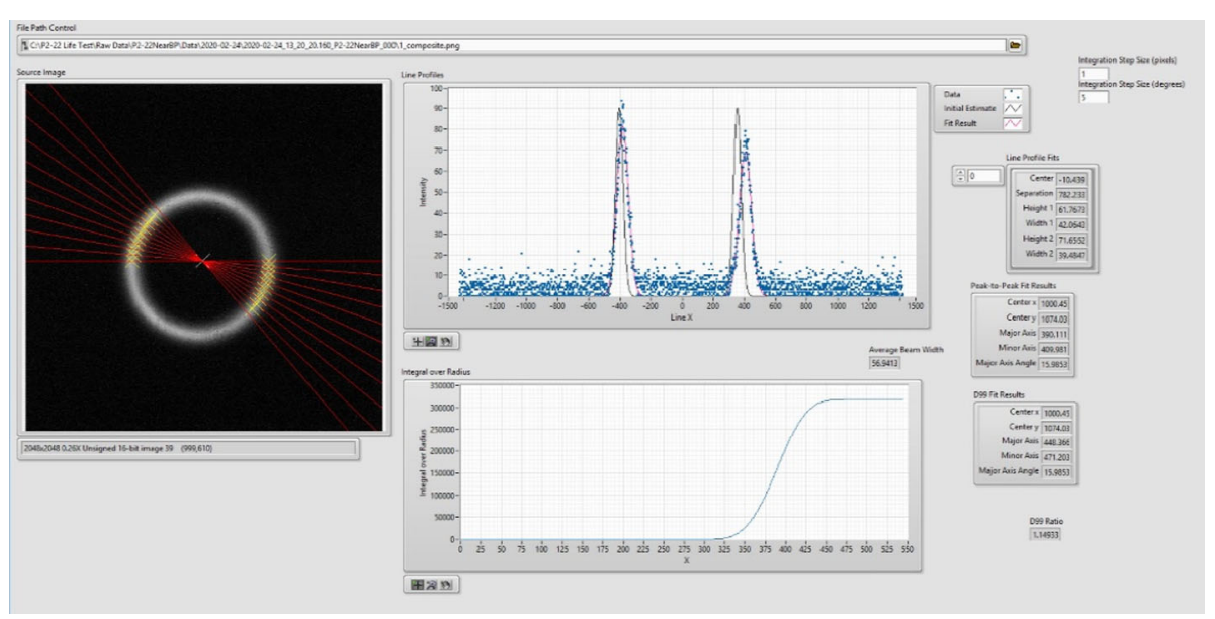

Fig. 38 Laser beam profile analysis software, where spatial dimensions are determined by numerous iterative stages as described in the text

diagnostic configuration, the input and output energy for every laser pulse was recorded with a time resolution of $0.5 \mu \mathrm{s}$.

\subsubsection{Beam Profile Analysis}

Prior to data acquisition, the properties of the beam profiler assembly were determined. The camera imaging optics were adjusted to focus on the BK7 slide with minimal distortion across the active space. The distance from laser output to the BK7 slide was measured, along with scale factors to convert images from pixel space on the sensor into distance space on the BK7 slide.

At the time of data acquisition, the raw image was background-subtracted from a previously-captured dark image. When the multiple images were being accumulated, background-subtracted images were summed and stored in a higher bit-depth image for better SNR.

The fitted ellipse provided information on the "peak-to-peak" diameter $\left(D_{P P}\right)$ of the annulus. In addition to this, the diameter for which $99 \%$ of the laser intensity falls ( $\left.\mathrm{D}_{99}\right)$ was determined by performing a numerical integration of the data (Fig. 38). This required interpolations and coordinate transformations to effectively perform, starting by generating a large collection of tightly-spaced line profiles, interpolating the data so each line profile matched with the same point density and peak-to-peak distance in line-profile-space, then summed over an elliptical coordinate system to ensure correct integrated weight contributions for each constituent area.

\subsection{Spectral Mapping Tests}

In an initial effort to understand the interaction and orchestration of the $\mathrm{C} \& \mathrm{DH}$, the scanner, the laser operations, the ACI focusing, and the spectrometer, an image of a BK7 resolution target was compared to that of a spectral map of the same target. Figures 39 and 40 shows the comparison of the ACI image and the resulting spectral map generated by the fluorescence emission from BK7. While the spectrometer mapping spatial resolution is expectedly 
Fig. 39 Flight unit ACI image of the $\mathrm{BK} 7$ resolution target used during thermal vacuum testing. For scale, the dots are $75 \mu \mathrm{m}$ apart

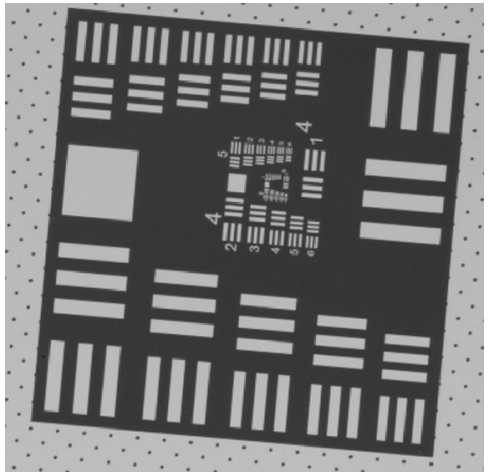

Fig. 40 Flight unit spectral map of the BK7 resolution target using laser as a light source

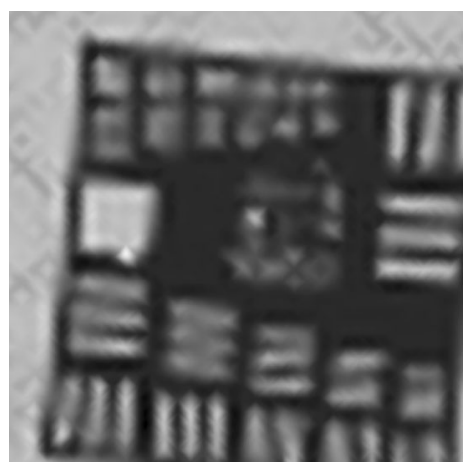

lower, this test demonstrated that the orchestration is working well and within requirements. Additionally, the orientation and overlap of the image and map was the first step toward being able to place spectral maps in context to images from ACI and WATSON images and ultimately, in spatial context with data from the other science and engineering imagers onboard Perseverance. This type of image-to-map correlation is also possible to characterize on the surface of Mars using the "maze" target on the SCT.

\section{SHERLOC Performance}

The SHERLOC science investigation objectives for spatially resolving organics at $1 \times 10^{-3}$ $\mathrm{wt} / \mathrm{wt}$ with bulk organics detections of $1 \times 10^{-5} \mathrm{wt} / \mathrm{wt}$ drove a large fraction of the in SHERLOC instrument design as well as the modes in which it will be operated on Mars.

\subsection{FM Acceptance Testing and Spectra}

To test the integrated SHERLOC instrument performance fully, the system was placed within a thermal-vacuum chamber at JPL (previously used for a number of prior Mars cameras), along with series of remotely accessible targets placed in front of the spectroscopy boresight. The targets included a subset of those from the SCT and a set of targets referred to as the thermal-vacuum (TVAC) palette, which included a carbonaceous chondrite (Allende), resolution targets and Raman and fluorescence standards (Fig. 41). Table 6 lists the 


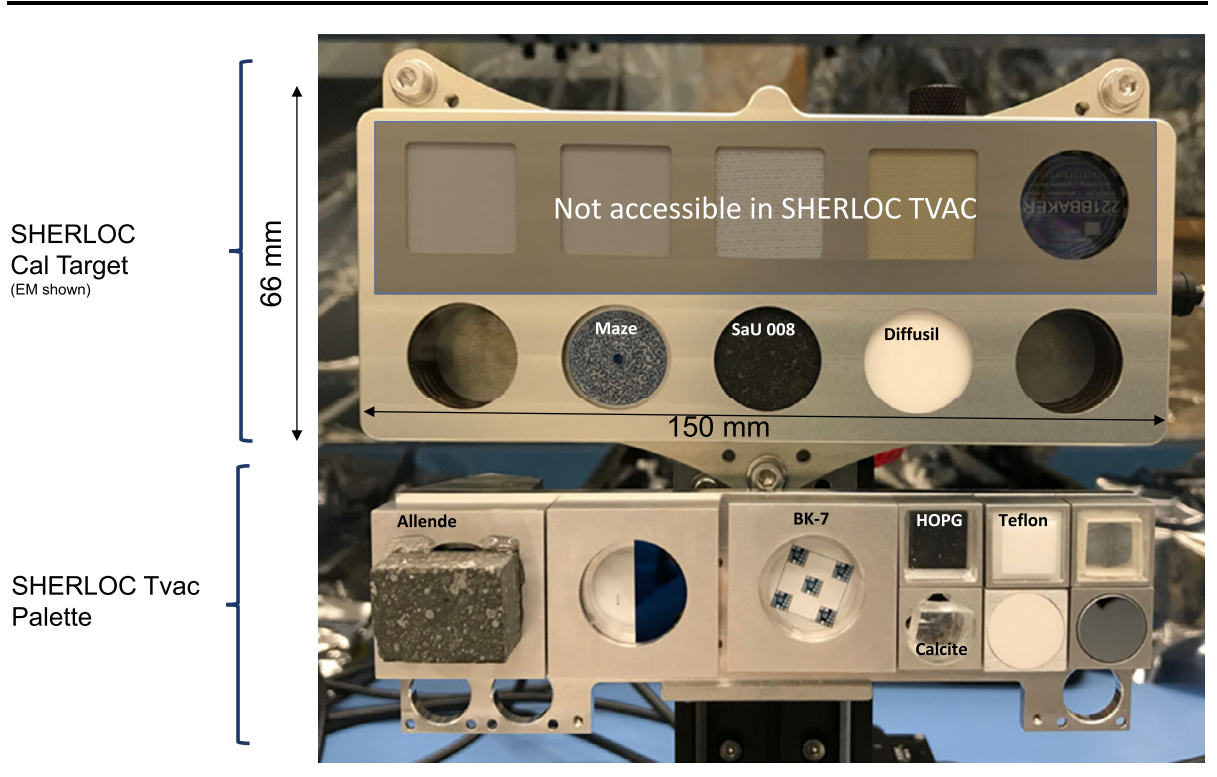

Fig. 41 Photograph of the SHERLOC Thermal-Vacuum (TVAC) test palette consisting of the SHERLOC Calibration Target (SCT) and a series of targets used to assess SHERLOC instrument performance. The SCT shown is the engineering model (EM), however, the original flight model (FM) was used for testing

Table 6 Targets used for SHERLOC thermal-vacuum (TVAC) testing, and purpose

SHERLOC TVAC Targets used for instrument level performance

\begin{tabular}{lll}
\hline Targets on SCT & Purpose & References \\
\hline SHERLOC Maze Target & Spectral and spatial resolution & \\
SaU 008 Meteorite & $\begin{array}{l}\text { Demonstration of organic and mineral } \\
\text { detection on Mars relevant samples }\end{array}$ & Grady et al.2004 \\
Diffusil Target & $\begin{array}{l}\text { Diffuse white balance and fused silica } \\
\text { Raman signature }\end{array}$ \\
\hline
\end{tabular}

Targets on TVAC Palette (Labeled on Fig. 41)

Allende Meteorite

BK7 Resolution Target

Highly Ordered Pyrolytic Graphite (HOPG)

Teflon

Calcite
Natural organic standard with $1 \times 10^{-5}$ Jarosewich et al. 1987 $\mathrm{wt} / \mathrm{wt}$ organics in a mineral matrix

Imaging resolution, mapping resolution, laser diameter, boresight alignment, image to map correlation.

Carbon standard for condensed carbon

Spectral standard to calibrate Raman spectral range and assess resolution

Spectral standard to calibrate Raman spectral range and assess resolution

targets used for the performance evaluation and their purpose. The SBA was located external to the chamber. 


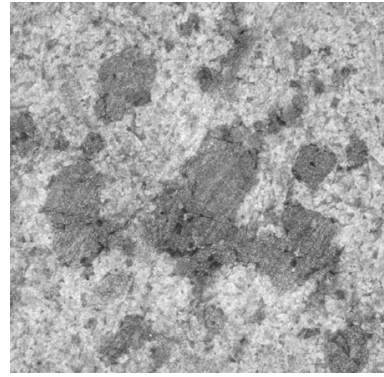

$\mathrm{ACl}$ image

$10.1 \mu \mathrm{m} / \mathrm{pix}$

FOV: $7 \times 7 \mathrm{~mm}$

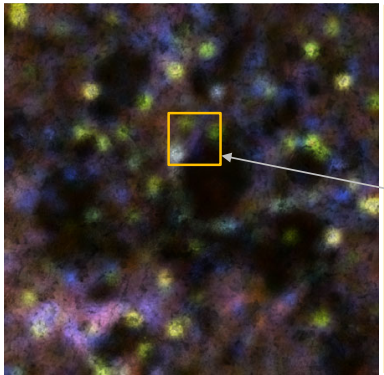

DUV Fluorescence Map

$200 \mu \mathrm{m} / \mathrm{pix}$

FOV: $7 \times 7 \mathrm{~mm}$

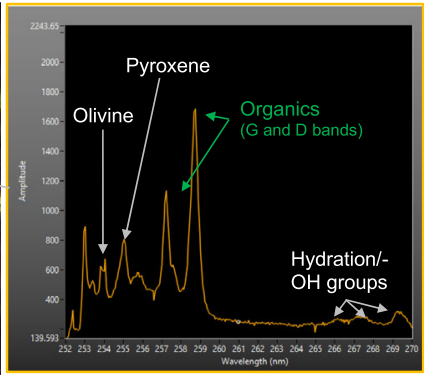

DUV Raman Analysis

$100 \mu \mathrm{m} /$ pix (avg)

FOV: $1 \times 1 \mathrm{~mm}$

Fig. 42 SHERLOC flight unit results from a single placement on the SaU 008 Martian meteorite under Mars pressure (nitrogen) and temperature

To demonstrate that the flight unit SHERLOC met the required performance, 14 $\mathrm{ACI} /$ spectroscopy and 9 WATSON requirements were assessed. Of these, the key requirements are described below.

\subsection{Single Placement}

One of the key aspects of the SHERLOC spectrometer and ACI is that it must be capable of achieving all science objectives at a given target on Mars using a single rover arm placement. This requires that the instrument detect the following with an SNR $>3$ within a nominal 60 minute operation:

1. aromatic organics at $1 \times 10^{-5} \mathrm{wt} / \mathrm{wt}$ over the map area;

2. aliphatic, aromatic and condensed carbon class of organics $1 \times 10^{-3} \mathrm{wt} / \mathrm{wt}$ within $100 \mathrm{um}$ areas; and

3. minerals at the $50 \mu \mathrm{m}$ grain size

Using the SaU 008 meteorite, courtesy of the Natural History Museum (London, UK), a Day-In-The-Life (DITL) measurement was conducted as an example of a nominal operation. Described in detail in Sect. 10, this consists of a Survey Scan over a 7 x $7 \mathrm{~mm}$ area with $200 \mu \mathrm{m}$ separation between points, with 10 pulses per point. This was followed by a detailed scan over a $1 \times 1 \mathrm{~mm}$ area, located within the initial survey map, with $100 \mu \mathrm{m}$ separation between points, and 300 pulses per point. The resulting spectral maps, image, and spectrum are shown in Fig. 42.

These results show that the condensed carbon ( $G$ and $D$ bands) and fine-grained minerals in the SaU 008 meteorite are detectable at SNR values greater than 10:1 within the required duration. It should be noted that while SHERLOC was not designed to detect silicates, these results demonstrate the capability exists.

In addition to the DUV Raman analysis, the DUV fluorescence map also highlights the spatial heterogeneity of organics in the sample within a $7 \times 7 \mathrm{~mm}$ region. While the autonomous selection of a region of interest was not incorporated in this test, the fluorescence map suggests that the organic concentration (purple) is higher in the lower left quadrant. It can also be observed that large, dark olivine phenocrysts $\left(\mathrm{Fo}_{64-71}\right)$ are devoid of organics and that the "green" fluorescence features are uniquely observed at the boundaries of these large phenocrysts. At the time of this writing, our data analysis is on-going as to the nature of these bright regions. 


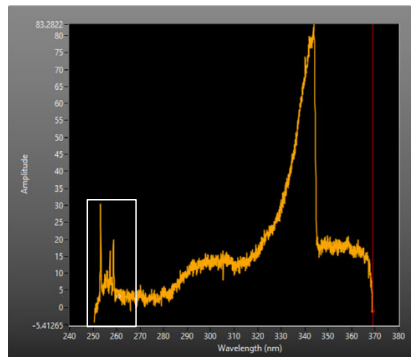

Fluorescence Average Spectrum $200 \mu \mathrm{m} /$ pix

FOV: $7 \times 7 \mathrm{~mm}$

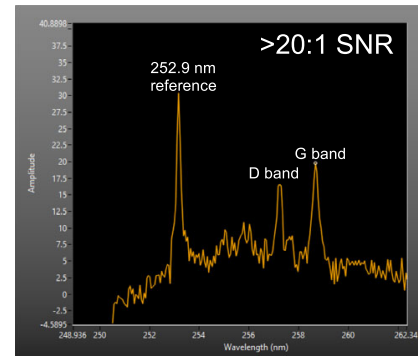

Raman Average Spectrum $200 \mu \mathrm{m} / \mathrm{pix}$ FOV: $7 \times 7 \mathrm{~mm}$

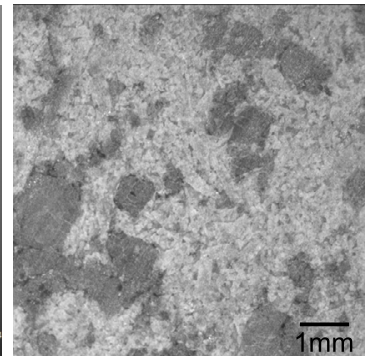

ACl image

$10 \mu \mathrm{m} / \mathrm{pix}$

FOV: $7 \times 7 \mathrm{~mm}$

Fig. 43 SHERLOC flight model bulk analysis of SaU 008 meteorite over a $7 \times 7 \mathrm{~mm}$ area shown in the ACI (right). The fluorescence spectrum (left) has a discontinuity as the latter portion of the fluorescence spectrum was acquired at a lower gain. The Raman spectral region (middle) has been expanded to observe the D and $\mathrm{G}$ organic bands from condensed carbon. Note the $252.9 \mathrm{~nm}$ line is an internal reference from the DUV laser used for wavelength calibration

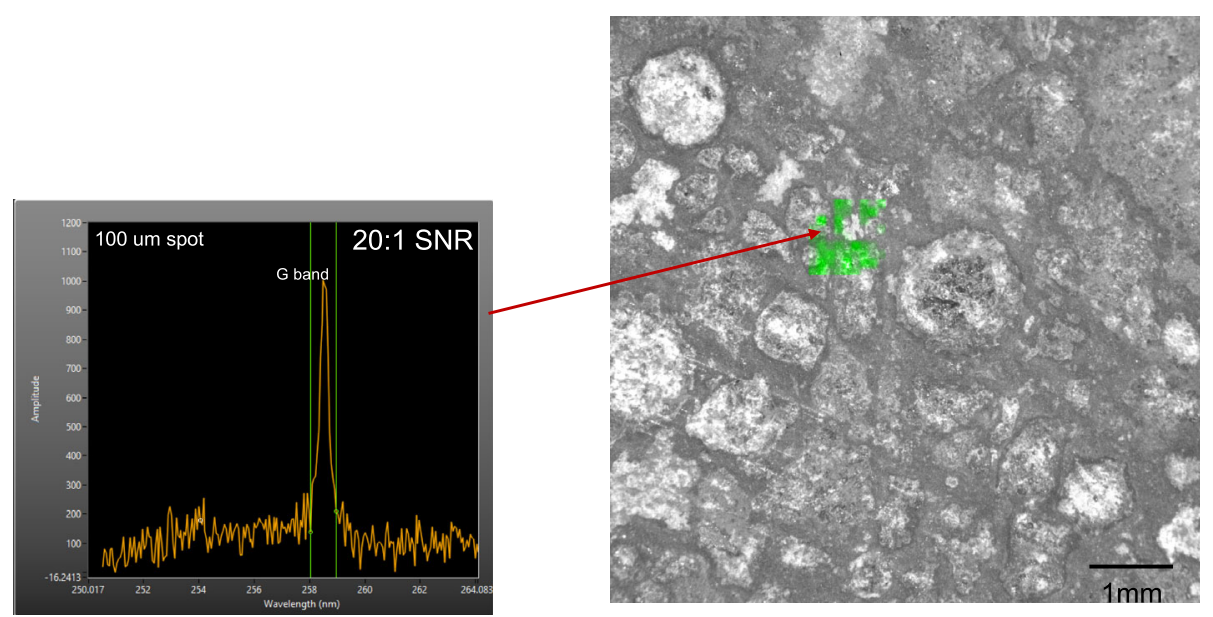

Fig. 44 Using SHERLOC's flight unit operated in Detail Mode (1x1 mm map with $100 \mu \mathrm{m}$ steps and 300 pulses/pt) the $\mathrm{G}$ band is prominently shown and mapped to the matrix of the Allende meteorite

\subsection{Bulk Organics and Spatially Resolved Organic Detection}

Using the SaU 008 target, SHERLOC used the survey map to demonstrate bulk organic detection with DUV Raman of condensed carbon. For SaU 008, the bulk carbon concentration is $8 \times 10^{-6} \mathrm{wt} / \mathrm{wt}$ (Grady et al. 2004) and the observed Raman SNR, averaged over the survey map ( 10 pulses/point) is $>20: 1$ where the fluorescence SNR is $>2 x$ higher (Fig. 43). The data were acquired over a period of about $\sim 10$ minutes.

In addition to the bulk analysis, the Allende meteorite was mapped over a $1 \times 1 \mathrm{~mm}$ area with $100 \mu \mathrm{m}$ steps to assess SHERLOCs ability to spatially resolve organics. As observed Fig. 44, the $2 \times 10^{-3} \mathrm{wt} / \mathrm{wt}$ bulk organics concentration (Jarosewich et al. 1987) was detected, as per the D and $\mathrm{G}$ band from macromolecular carbon, and spatially resolved at $100 \mu \mathrm{m}$ steps with a 20:1 SNR using the deep UV Raman. 

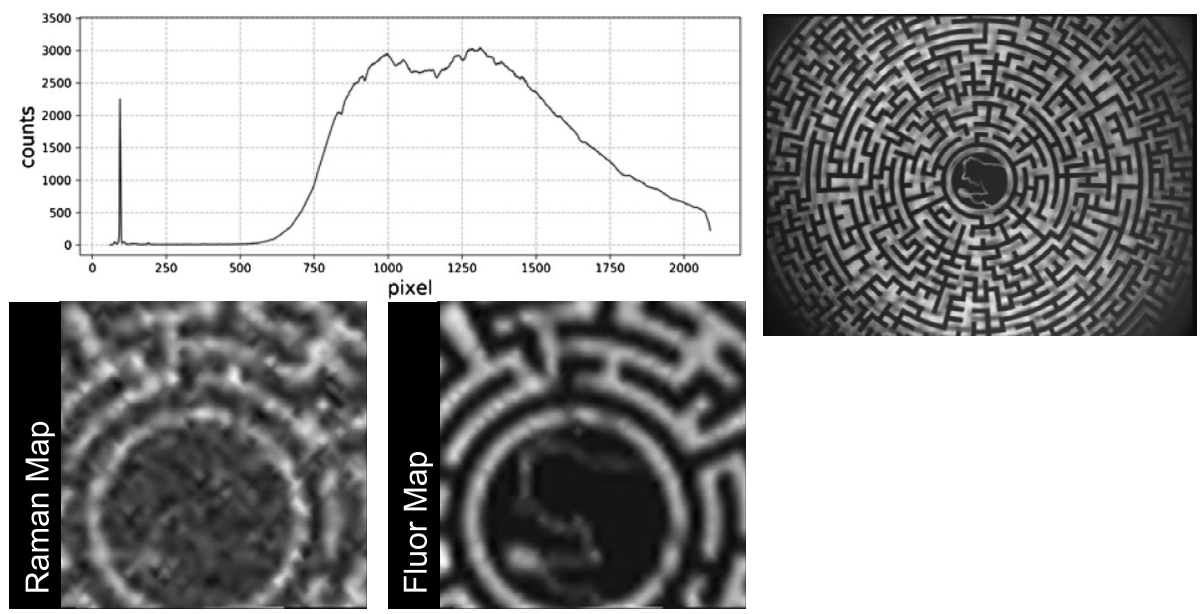

Fig. 45 SHERLOC flight unit analysis of the maze target showing a spatial mapping resolution of $\leq 100 \mu \mathrm{m}$
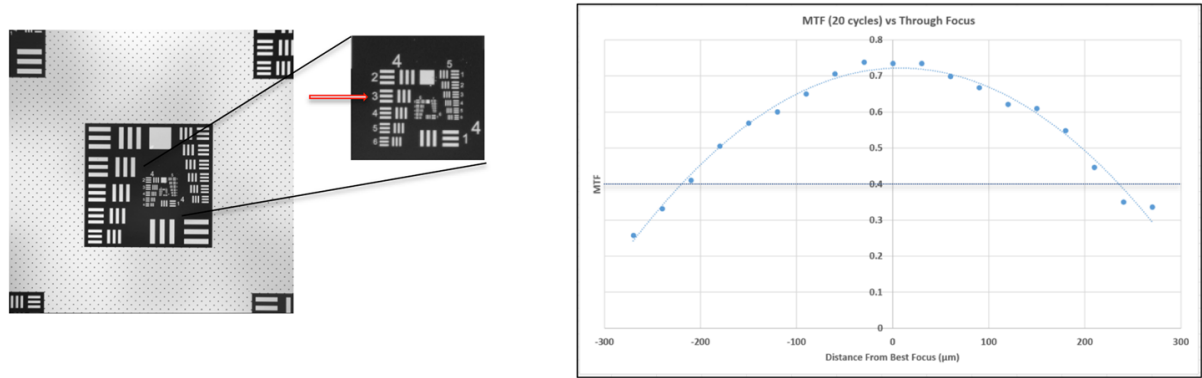

Fig. 46 Flight unit ACI image of the BK7 resolution target. The arrow indicated the target for 20 line pairs $/ \mathrm{mm}$. At best focus, this generates an MTF of 0.7 , and only reduces to 0.4 at $\pm 200 \mu \mathrm{m}$ from best focus

\subsection{Spectral Mapping Spatial Resolution}

One of the unique capabilities of SHERLOC is the ability to spatially resolve the organic and mineral distributions on a geological surface and correlate it with morphological features. As shown in Fig. 42, with the SaU 008 meteorite, and the Strelley Pool stromatolite observations in Fig. 4, this provides not only a means to spatially deconvolve the chemical signatures, but also leads to an understanding of possible formation/deposition mechanisms.

We used the SCT "maze" target (Fig. 26b) to demonstrate the $\leq 100 \mu \mathrm{m}$ spatial mapping capability of SHERLOC. This target will be used on Mars for calibration as well as to monitor instrument health and alignment throughout the duration of the mission. The target pattern consists of $200 \mu \mathrm{m}$ wide chrome bars that are separated by spaces of $200 \mu \mathrm{m}$ width. The area between the chrome bars generates both a strong Raman signature at $\sim 1600 \mathrm{~cm}^{-1}$ as well as a strong fluorescence spectrum at $360 \mathrm{~nm}$. As observed in Fig. 45, a 3.5 x $3.5 \mathrm{~mm}$ map with $100 \mu \mathrm{m}$ steps around the center of the maze generates the expected maze pattern and in addition the $50 \mu \mathrm{m}$ lines from the silhouette of the SHERLOC logo can be observed in the fluorescence maps. 


\subsection{ACI Pre-Launch Characterization and Calibration}

\subsubsection{Image Range and Scale Relations}

Image Scale and Working Distance from Focus Position The ACI is a fixed focus imager with a $48 \mathrm{~mm}$ nominal standoff and a $0.74 x$ magnification of the image plane to the detector FPA. This equates to an image scale of $10.1 \mu \mathrm{m} /$ pixel and a theoretical FOV of $16.16 \mathrm{~mm} \times 12.12 \mathrm{~mm}$. However, over the $7 \times 7 \mathrm{~mm}$ center of the detector (or $11.7 \mathrm{~mm}$ diameter), the image is unvignetted and the distortion is minimized with the ability to resolve $<30 \mu \mathrm{m}$ line pairs (Fig. 46).

Since the ACI image path (Fig. 13) is co-boresighted to the laser and observes the target through scanner mirror, the laser is always aligned to the center of the ACI image (within the co-boresighting error). Thus, as the laser spot is rastered over the target surface, an ACI image can be collected, each time, for registration purposes. However, as the scanner moves the imager off center, the image becomes distorted. Therefore, all spatial resolution values and FOV's for the ACI assume that the scanner is at the center of the boresight.

While the ACI is a fixed focus imager, the objective lens can adjust to the working distance once SHERLOC is positioned by the robotic arm by $\pm 8.35 \mathrm{~mm}$; enabling a working distance range of 39.7 to $56.4 \mathrm{~mm}$. This allows the ACI to find focus when placed at the target within the robotic arm positioning uncertainties. However, given the offset between the imager and the spectrometer ideal focus, the total focusable range for spectral maps is reduced to 40.1 to $56 \mathrm{~mm}$.

ACI stepper motor counts, which are a function of ACI Objective lens focus, can be used to determine working distance for in-focus elements of a given image. The field of view (FOV), depth of field (DOF), and pixel scale are constant. The relationship between stepper motor count is highly linear and described by Eq. (1):

$$
d_{w}=0.005 m-20.34
$$

in which $m$ is the motor count and $d_{w}$ is the working distance in units of millimeters. The range of motor counts this applies to is from 12072 to 15270 , equating to a working distance of 40 to $56 \mathrm{~mm}$. Outside of this range, the cover is opening or closing.

Image Resolution over the Depth of Field In order to focus the SHERLOC spectrometer, the ACI image and spectrometer focus are at a known objective lens motor position offset. As such, the ACI can be used to focus on a target, and the spectrometer can be focused relative to that position $(\sim 700 \mu \mathrm{m})$. In addition, the ACI needs to have a spatial resolution that provides context as to where the spectra are acquired. With a projected spectrometer slit of $100 \mu \mathrm{m}$, the spatial resolution of the imager required is $\leq 50 \mu \mathrm{m}(20$ line pairs $/ \mathrm{mm})$ with an MTF (modulation transfer function) of $>0.4$.

To enable these, the ACI design resulted in a $\pm 200 \mu \mathrm{m}$ depth of field, defined by $>0.4$ MTF at 20 line pairs/mm with a best focus MTF of 0.7 (Fig. 46). This is smaller than the DOF of the spectrometer $( \pm 500 \mu \mathrm{m})$, in which, at best focus, the spatial resolution of the $\mathrm{ACI}$ is at $\sim 30 \mu \mathrm{m}$ (33 line pairs $/ \mathrm{mm})$.

\subsection{WATSON Pre-Launch Characterization and Calibration}

The following sections provide an overview of the WATSON camera characterization and calibration results. Details can be found in Edgett et al. (2019) 


\subsubsection{Image Range and Scale Relations}

Image Scale and Working Distance from Focus Position Each MAHLI and WATSON camera head has a different as-built relation between the instrument-reported focus position, working distance, and image scale. Focus position is reported by the instrument as a function of the incremental stepping of the Cobham stepper motor (Edgett et al. 2012). At the starting point, a motor count $(m)$ of 0 , the transparent dust cover is closed and the lens is in focus for targets at the closest possible working distance.

The relations between focus position (motor count), working distance, and image pixel scale for WATSON were determined empirically by autofocusing on planar targets, placed parallel to the WATSON CCD, located at different working distances over the full range of focus. Results were obtained for motor count positions with the dust cover open $\left(m_{\text {open }}\right)$ and closed $\left(m_{\text {closed }}\right)$. These pre-launch laboratory measurements found that the relation between pixel scale ( $\mathrm{p}$, in units of $\mu \mathrm{m} / \mathrm{pixel})$ and working distance $\left(d_{w}\right.$, in units of $\left.\mathrm{cm}\right)$ for the WATSON onboard Perseverance is determined by:

$$
p=6.7593+3.658 d_{w} .
$$

Working distance $\left(d_{w}\right)$, in turn, is estimated from the focus position reported as a motor count $(m)$. We determined that the minimum working distance at which Perseverance's WATSON can focus is $1.78 \pm 0.1 \mathrm{~cm}$. This results in images of $13.1 \pm 0.1 \mu \mathrm{m} / \mathrm{pixel}$. This is a higher spatial resolution than the MSL MAHLI can achieve. However, based on MAHLI experience (Yingst et al. 2016), the camera will likely never be positioned this close to a target on Mars. Typical Mars surface high resolution images will be in the 16-22 $\mu \mathrm{m} / \mathrm{pixel}$ range (Yingst et al. 2016). At the other end of the focus range, for targets farther than $~ 300$ $\mathrm{cm}$, WATSON images are at infinity focus when $m_{\text {open }}=12000$ and $m_{\text {closed }}=4890$. In the motor count range between 4890 and 12000, the dust cover is either opening or closing; in-focus images of targets at infinity distance can still be achieved over this range, which is not the case for MAHLI. WATSON performance in the "beyond infinity" range, and at the minimum working distance, are indicators that the WATSON optics outperform those of the MAHLI.

Typical WATSON imaging of geological and engineering targets will occur within the range between the minimum and infinity working distances (Yingst et al. 2016). For that range, our empirical measurements determined that the relation between dust cover open motor count $\left(m_{\text {open }}\right)$ and working distance $\left(d_{w}\right)$, in centimeters, is:

$$
d_{w}=\left(\mathrm{a} m_{\text {open }}^{-1}+\mathrm{b}+\mathrm{cm} \text { open }+\mathrm{d} m_{\text {open }}^{2}+\mathrm{e} m_{\text {open }}^{3}\right)^{-1}
$$

in which $\mathrm{a}=699788, \mathrm{~b}=-217.911, \mathrm{c}=2.56035 \times 10^{-2}, \mathrm{~d}=-1.35136 \times 10^{-6}$, and $\mathrm{e}$ $=2.71652 \times 10^{-11}$. Equation (3) applies only to the WATSON onboard Perseverance, only when the dust cover is open, and only when $m_{\text {open }}$ is between $\sim 12228$ and 15420 . The relation between working distance and motor count for the dust cover closed case is best described by the following relationship between dust cover open $\left(m_{\text {open }}\right)$ and dust cover closed motor counts $\left(m_{\text {closed }}\right)$ :

$$
m_{\text {closed }}=16890-m_{\text {open }}
$$

Equation (4) applies only to the WATSON onboard Perseverance, only when the dust cover is closed, and only when $m_{\text {closed }}$ is between $\sim 1470$ and $\sim 4662$. Equations (3) and (4) should only be used only for cases in which $d_{w}$ is in the 1.8 to $\sim 220 \mathrm{~cm}$ range. The distance to targets outside this range can not be determined from motor count. 
Depth of Field Depth of field (DOF) is the distance between the nearest and farthest infocus feature in a given image. DOF is somewhat subjective, as items that might appear to be in focus to one observer might seem somewhat blurry to another. Uncertainty in the relations between $d_{w}$ determined from $m_{\text {open }}$ or $m_{\text {closed }}$ can be estimated using knowledge of the WATSON DOF. As illustrated by Ghaemi (2009), the DOF at small working distances $(<12 \mathrm{~cm})$ was defined as the range over which the lens MTF is at $>50 \%$ of its peak infocus value. For targets at a greater range $(>12 \mathrm{~cm})$, DOF is estimated using the hyperfocal distance $\left(d_{h}\right)$ equation,

$$
d_{h}=\left(f_{e}^{2} / f_{n} c_{c}\right)+f_{e}
$$

in which $f_{e}$ is the lens effective focal length, $c_{c}$ is the circle of confusion, and $f_{n}$ is the focal ratio (f/number). For WATSON, $f_{e}$ and $f_{n}$ change as a function of focus position. We assume $c_{c}$ is a diameter equivalent to two pixel dimensions, (i.e., $14.8 \mu \mathrm{m}$ at the detector surface); this provides the best match to the 50\% MTF fall-off method at $11.25 \mathrm{~cm}$ and is consistent with observations of blurring of near and far objects in MAHLI portraits of the Curiosity rover (e.g., Fig. 23H). When the WATSON dust cover is open, we estimate that the relation between $m_{\text {open }}$ and the range, in centimeters, to the near $\left(d_{\text {near }}\right)$ and far $\left(d_{\text {far }}\right)$ depth of field, also in centimeters, is:

$$
d_{\text {near }} \text { or } d_{f a r}=\left(\mathrm{a} m_{\text {open }}^{-1}+\mathrm{b}+\mathrm{c} m_{\text {open }}+\mathrm{d} m_{\text {open }}^{2}+\mathrm{em}_{\text {open }}^{3}\right)^{-1},
$$

in which, for $d_{\text {near }}, \mathrm{a}=758336, \mathrm{~b}=-235.879, \mathrm{c}=2.76772 \times 10^{-2}, \mathrm{~d}=-1.45801 \times 10^{-6}$, and $\mathrm{e}=2.92292 \times 10^{-11}$; and, for $d_{\text {far }}, \mathrm{a}=755933, \mathrm{~b}=-234.525, \mathrm{c}=2.74318 \times 10^{-2}$, $\mathrm{d}=-1.43997 \times 10^{-6}$, and $\mathrm{e}=2.87582 \times 10^{-11}$. Ultimately, Eqs. (3), (4), and (6) provide an estimate of working distance $\left(d_{w}\right)$ and working distance uncertainty ( $d_{\text {near }}$ and $\left.d_{f a r}\right)$ as a function of focus motor count ( $m_{\text {open }}$ and $\left.m_{\text {closed }}\right)$.

Image Resolution and Contrast (System MTF) Pixel scale (Eq. (2)) concerns the relation between the surface area of a target detected by a given pixel in an image. Resolution, the smallest extent of an areal feature that can be identified and measured accurately and with certainty, is a related matter. Determination of a camera's Modulation Transfer Function (MTF) provides a measure of resolution and contrast capability. We determined WATSON system MTF at two focus positions by imaging a slanted edge black and white target. The image colors produced by the CCD's Bayer pattern microfilters were interpolated before analysis. Near minimum focus $\left(d_{w}=1.84 \mathrm{~cm}\right)$, the system MTF at Nyquist frequency was found to be $17.3 \%$. At a large working distance $\left(d_{w}=326 \mathrm{~cm}\right)$, approaching infinity focus, the system MTF at Nyquist frequency was measured as $22.1 \%$. Both values met and exceeded a requirement of $>15 \%$ over the full WATSON focus range. Additional images regarding the flight model WATSON performance can be found in Edgett et al. 2019).

\subsubsection{System Image Detection Characterization}

System Scale Factor, Full Well, Read Noise, Linearity We characterized the scale factor (electrons, $\mathrm{e}^{-}$, per DN), read noise $\left(\mathrm{e}^{-}\right)$, and full well capacity $\left(\mathrm{e}^{-}\right)$of the WATSON signal chain by imaging a diffuse illuminated integrating sphere target, with the instrument dust cover closed, and by analysis of photon transfer curves (PTC) using the technique described by Janesick et al. (1987). We used images acquired at room temperature in a cleanroom laboratory setting on 03 October 2019 and found the PTC and linearity results to be: scale factor $15.84 \mathrm{e}^{-}$per DN, read noise $18.7 \mathrm{e}^{-}$, full well 24,507 e-. Addition images regarding the flight model WATSON performance can be found in Edgett et al. 2019). 
Dark Current and Bias Dark current is the accumulation of charge within the detector from non-photoelectric phenomena, mostly thermally generated electrons. Typical WATSON camera head operations on Mars are intended to occur at temperatures in the $-40{ }^{\circ} \mathrm{C}$ to $+40{ }^{\circ} \mathrm{C}$ range. Under these conditions, from instrument stand-alone thermal vacuum testing, Perseverance's WATSON exhibits negligible noise from dark current for 250 millisecond exposures over this temperature range. The dark bias is a noiseless DC offset in the signal level that we determined from the data to be a 12-bit DN value of $\sim 119$. Based on this result, as implemented onboard the ACI/WATSON DEA, a bias of 120 is removed from WATSON images during commanding. The bias is reported in the image header. Addition details regarding the flight model WATSON performance can be found in Edgett et al. 2019).

Particulates on the Detector Despite extreme measures to avoid contamination of the detector, WATSON images exhibit dark spots that result from the presence of tiny particles on the CCD. Most of these particles are $\leq 1$ pixel $7.4 \mu \mathrm{m}$, in size); the largest (pre-launch) cover areas of $<5$ by 3 pixels. Some particles are opaque, others are transparent, either because of their size, shape, or inherent transparency. The MSL MAHLI CCD, operating on Mars, has similar particulates; they do not change positions over time and thus can be used as fiducial or reseau marks useful for understanding camera motion while imaging at a single camera position or differences in robotic arm placement as a result of repeated imaging at different times (Edgett et al. 2015). These blemishes are small enough that flat fielding can cosmetically remove them. It should be noted that the ACI and the SCCD detectors were less prone to particulate deposition as they are protected well within the instrument and away from any mechanisms. Additional images regarding the flight model WATSON particulates can be found in Edgett et al. 2019).

\subsubsection{Radiometric Response}

System Flat Field Flat field products are used to account for the effects of the camera system on the image detected, including: (1) pixel-to-pixel variations in response; (2) the impact on image detection induced by the nature of the optics, focal plane assembly, and their housings; and (3) particulates in the optical path. For a spacecraft camera, it is commonly ideal to create a flat field product from observations acquired before launch, because, in some cases, the pre-launch flat field products might be the only such products that can ever be produced if it is difficult or impossible to obtain post-launch flat field observations. In other cases, there are methods for obtaining flat field observations in flight or on the surface of a planetary body. For a camera on Mars, the latter can be performed through dedicated observations of the sky (e.g., Herkenhoff et al. 2006).

For an instrument like WATSON, with its transparent dust cover, it might seem prudent to obtain pre-launch flat field data with the dust cover open and closed. However, the MSL experience is that the dust cover will likely become coated with a thin film of very fine dust during the rover's terminal descent in Jezero crater on Mars, and that dust will remain on the cover throughout the mission (Edgett et al. 2015). Thus, pre-launch flat field data were only acquired with the WATSON dust cover open. The flat field image are of a uniformlyilluminated (as close to uniform as practicable) Spectralon ${ }^{\mathrm{TM}}$ target. With the WATSON camera head was pointed at the target and the dust cover open, images were acquired at 82 $m_{\text {open }}$ focus positions over the full range of focus. Examples are shown in Fig. 47. A good test of the success of a flat field product is that the images should form a seamless mosaic (Fig. 48). It is important to note, however, that after these data were acquired, additional particulates accumulated on the CCD and these are not documented in the pre-launch flat 

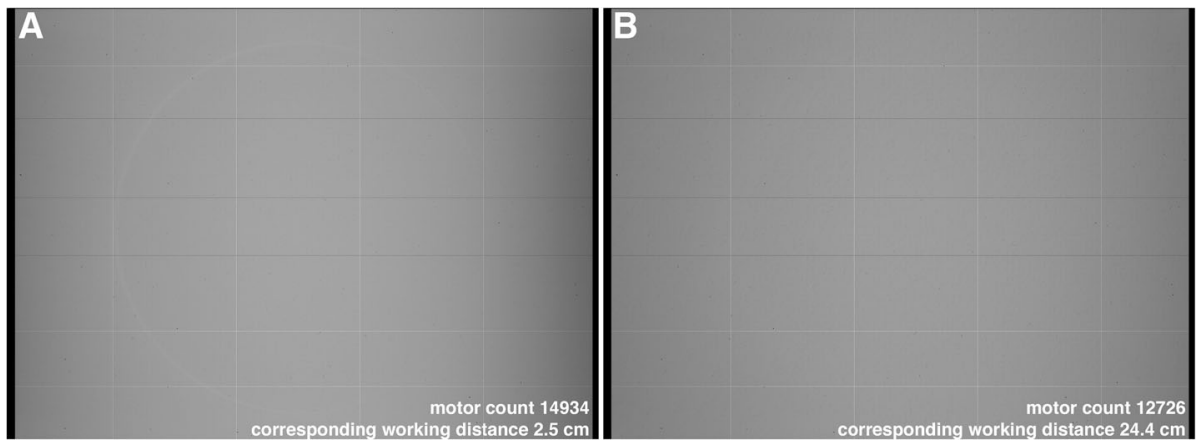

Fig. 47 Example WATSON flat field products from data acquired during stand-alone instrument testing on 04 October 2019. (A) Close range (small working distance) image showing bright "stray light" ring around the center of the frame caused by reflections within the instrument. (B) Flat field image corresponding to a lower spatial resolution context image that WATSON would acquire on Mars; note the bright ring is absent as focus shifts toward greater working distances

Fig. 48 Demonstration of seamless mosaicking using flat fielded WATSON images of a basalt cobble collected by M.C. Malin in Iceland (also imaged by MSL cameras; Malin et al. 2017). The mosaic consists of three images acquired during stand-alone cleanroom testing on 09 October 2019

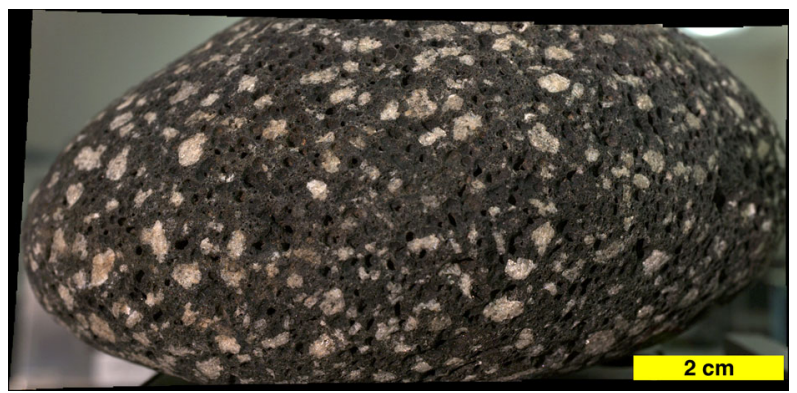

\begin{tabular}{lccc}
\hline band & predicted (e-) & measured (e-) & difference \\
\hline red & 14958 & 14769 & $-1.3 \%$ \\
green & 9145 & 8557 & $-6.4 \%$ \\
blue & 4437 & 4130 & $-6.9 \%$ \\
\hline
\end{tabular}

Table 7 Perseverance WATSON signal (electrons, e-) performance as a function of Bayer red, green and blue relative to prediction (assumes lens at $\sim \mathrm{f} / 9.8$ )

field data. This means that acquisition of sky flat field images on Mars will be of primary importance.

Absolute and Relative Radiometric Response The WATSON absolute radiometric response accounts of application of dark current and bias, flat fields, system spectral throughput, and calibrated measurements of the signal received by the red, green, and blue (RGB) microfilters of the WATSON CCD.

During pre-launch testing, we confirmed that WATSON meets expectations based on lens transmission and CCD quantum efficiency (QE). This was confirmed by imaging an integrating sphere and a calibrated Quartz Tungsten Halogen (QTH) lamp traceable to National Institute of Standards and Technology (NIST) standards. Using data obtained on 03 October 2019 (dust cover closed, exposure 30 milliseconds, minimum working distance focus), the signal level was within $<10 \%$ of prediction for all three Bayer filter bandpasses (Table 7). 
Table 8 Perseverance WATSON fields of view (FOV) over the full focus range

\begin{tabular}{llll}
\hline Focus position & diagonal & $\begin{array}{l}\text { horizontal } \\
\text { (CCD columns) }\end{array}$ & $\begin{array}{l}\text { vertical } \\
\text { (CCD rows) }\end{array}$ \\
\hline $\begin{array}{l}\text { Close focus (minimum } \\
\text { working distance) }\end{array}$ & $41.0^{\circ}$ & $32.8^{\circ}$ & $24.6^{\circ}$ \\
\begin{tabular}{l} 
Infinity focus \\
\hline
\end{tabular} & $39.0^{\circ}$ & $31.2^{\circ}$ & $23.4^{\circ}$ \\
\hline
\end{tabular}

Color Adjustment Coefficients White balance or color adjustments are generally a matter of individual preference or objective. The main reason one might perform a color or white balance adjustment to an image is to accommodate illumination source "temperatures" (i.e., the light source hue as compared with the temperature of a blackbody radiator matching that hue). Consumer digital cameras, for example, commonly have built-in capabilities that allow the user to adjust for differences in illumination conditions, such as a bright, sunny day, an overcast day, or indoor illumination by different types of lamps. The solar illumination reaching the Martian surface, through its atmosphere and with a given opacity at a given moment in time, differs from the terrestrial experience. One motivator for performing a color adjustment on a WATSON image, then, is to ensure that the geologic features observed appear as they might to a human eye working with the materials if they were in a laboratory or field setting on Earth.

For the MSL mission, color adjustment coefficients corresponding to images acquired through the lenses and Bayer pattern red, green, and blue microfilters of the MAHLI CCD were determined using a flight-fidelity copy of Curiosity's MAHLI, the testbed-MAHLI. This MAHLI camera head has been onboard the MSL testbed rover at JPL-Caltech throughout much of the MSL mission, although it also was used for a period in 2019-2020 in the Mars 2020 testbeds. The testbed-MAHLI was used to determine color adjustment coefficients because the instrument can be operated outside a cleanroom environment on Earth. In 2010, it was taken outside and used to image - under near-noon solar illumination on a clear, blue sky day - a target consisting of color and gray swatches identical to those of the MSL MAHLI Calibration Target on Mars (Edgett et al. 2012, 2015). From these data, we derived color correction factors (1.16 for red, 1.00 for green, and 1.05 for blue) based on examination of a $40 \%$ gray swatch on the target (Edgett et al. 2015). Because WATSON is a copy of the MSL MAHLI, with the same CCD and optics, these correction factors can similarly be applied to WATSON data.

\subsubsection{Geometric Characterization}

Field of View over Focus Range WATSON's field of view was empirically measured at close focus and infinity focus positions by imaging targets positioned at working distances of $1.8 \mathrm{~cm}$ and $>8 \mathrm{~m}$. The resulting fields of view are summarized in Table 8 . As expected, WATSON FOV decreases as working distance increases. Our analysis effort also resulted in the recognition that the horizontal, vertical, and diagonal fields of view (FOV) reported in the MSL MAHLI calibration report (Edgett et al. 2015) are in error and should be ignored.

Lens Distortion The WATSON (and MSL MAHLI) lens was designed to provide images exhibiting negligible distortion (Ghaemi 2009). The undistorted nature of WATSON images was confirmed during cleanroom testing by imaging a $50 \times 50 \mathrm{~mm}, 1 \mathrm{~mm}$ pitch grid target at a working distance of $3.36 \mathrm{~cm}(\sim 19 \mu \mathrm{m} / \mathrm{pixel})$ and demonstrating that grid dimensions at the center of the image matched grid dimensions at the four corners of the image. Addition details regarding the flight model WATSON performance can be found in Edgett et al. 2019). 
Fig. 49 Photographs of the targets used for the STT. The EM SCT and an STT custom palette were used for SHERLOC testing. The labeled items were the targets used

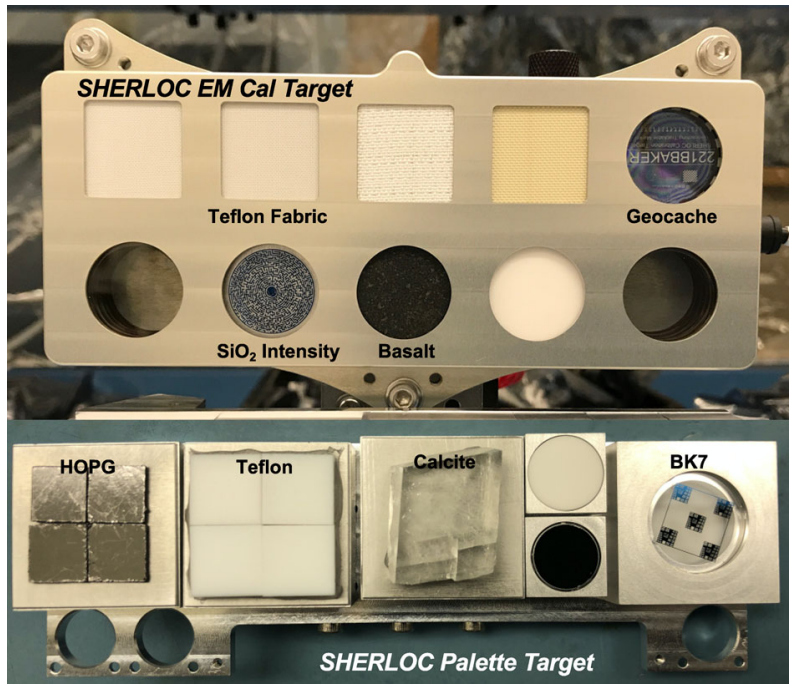

Camera Model A geometric camera model is a set of equations that transform a 3D point in space to a $2 \mathrm{D}$ position in an image (pixel location). The model can also be inverted to transform a pixel in the image to a set of points in space that would map to that pixel. The model contains the camera position and pointing vector, it also models lens distortion and the interior geometry of the instrument. A camera model facilitates accurate monoscopic and stereoscopic measurement of points in an image or images, precise mosaicking of images, and creation of linearized (i.e., geometrically corrected) products for which lens distortion has been removed. At the time of the writing, the CAHVOR camera model is in progress of being generated using data collected during ATLO testing before launch and will be included in a future report.

\subsection{Rover System Thermal Tests: SHERLOC Results}

In addition to SHERLOC instrument level tests, the STA was mounted on the turret at the end of the rover robotic arm (Fig. 8) and performance was tested in ATLO, especially at rover level System Thermal Testing (STT), and for EMI/EMC testing. These were the final tests to demonstrate operability with the rover and evaluate the electrical connections, commanding methodology, and overall instrument performance. Since SHERLOC's optical system is optimized for Mars ambient temperature/pressure, the STT tests provided the best insight on how SHERLOC will perform on Mars.

The EMI/EMC testing results revealed no issues aside from expected interference to the SHERLOC scanner from helicopter (Ingenuity) operations, and specific RIMFAX modes of operation (Balaram et al. 2018). In both cases, SHERLOC spectroscopy would not be operated during these instances.

For STT tests, much of the SHERLOC test targets were composed of the EM SCT and a palette with targets similar to those used for FM acceptance testing and instrument performance evaluation at the instrument level TVAC. However, to accommodate the arm positioning uncertainties, the palette target sizes were increased to $\geq 2 \mathrm{~cm}$ widths (Fig. 49). 

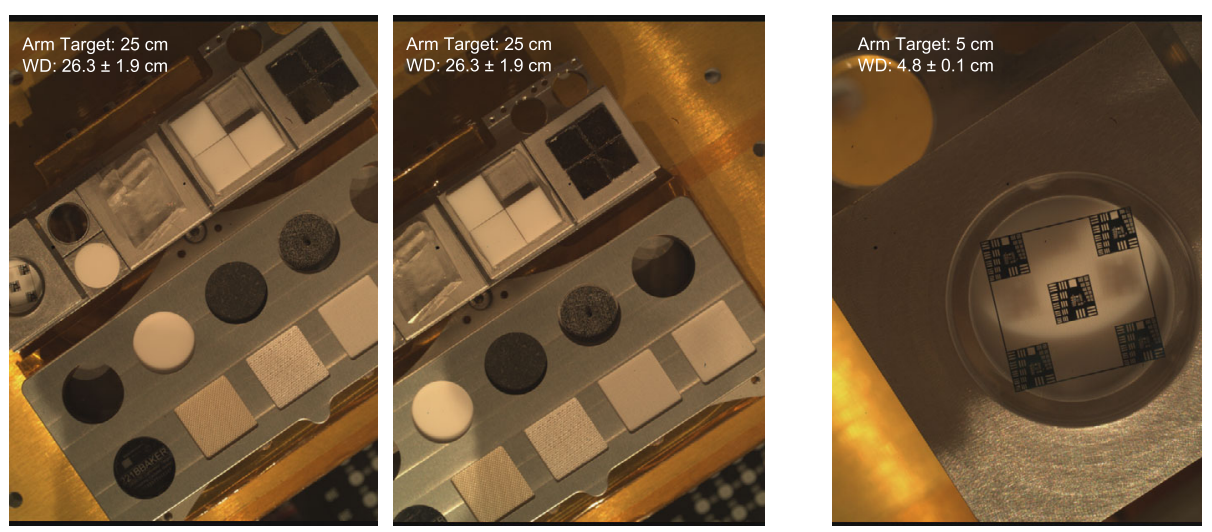

Fig. 50 Testbed-WATSON images of the SHERLOC targets in STT showing intended working distance ("Arm target") as compared with the working distance (WD) determined from WATSON focus position (reported as motor count)

\subsubsection{Arm Placement and Image Mosaics}

The initial STT effort focused on the ability of the robotic arm to accurately place SHERLOC at a specified target within the rover workspace. Given the focus range and field of view, SHERLOC spectroscopy and ACI imaging relies heavily on robotic arm performance. While it was designed to meet the expected errors, the tests in STT were the first demonstrations of placement of SHERLOC by the robotic arm. The initial tests used WATSON at 25 $\mathrm{cm}$ and $5 \mathrm{~cm}$ working distances (Fig. 50). Using the motor count position from these WATSON images, the distance to the target was assessed and compared to the distance at which the arm intended to place the camera. Note that the arm positions used pre-determined positions defined by laser metrology of the targets relative to the rover workspace. While this may suggest a performance that is better than the onboard engineering camera-based positioning, there are also uncertainties in the metrology and the target positions. As such these tests provide confidence in placement, but arm placement accuracies will only be truly understood on the surface of Mars.

As shown in Fig. 50, the arm positioned the WATSON boresight within the error of the depth of the field of the instrument's ranging capability. There are slight lateral shifts when observing the $5 \mathrm{~cm}$ target, however, this was well within the expected arm uncertainty and thus could be accommodated by SHERLOC. In addition to the distance to targets, the WATSON images acquired were part of an arm mosaic test (Fig. 51).

In addition to the WATSON images and mosaics, an ACI image of the BK7 resolution target was acquired. This is shown in Fig. 52 as an overlay on the WATSON image for comparison and to demonstrate the expected relative rotations between the detectors.

\subsubsection{SHERLOC Internal Calibration}

Before opening the dust cover of the spectroscopy boresight, an AlGaN target on the inside of the cover provides a means to test the functionality and performance of the spectrometer and laser, and provide for calibration of the laser energy and the spectrometer spectral position. The AlGaN material was provided by Texas Technology University where AlGaN 
Fig. 51 Testbed-WATSON mosaic acquired during STT showing the SHERLOC calibration targets. This includes the two $25 \mathrm{~cm}$ standoff images and the $5 \mathrm{~cm}$ standoff image from Fig. 50

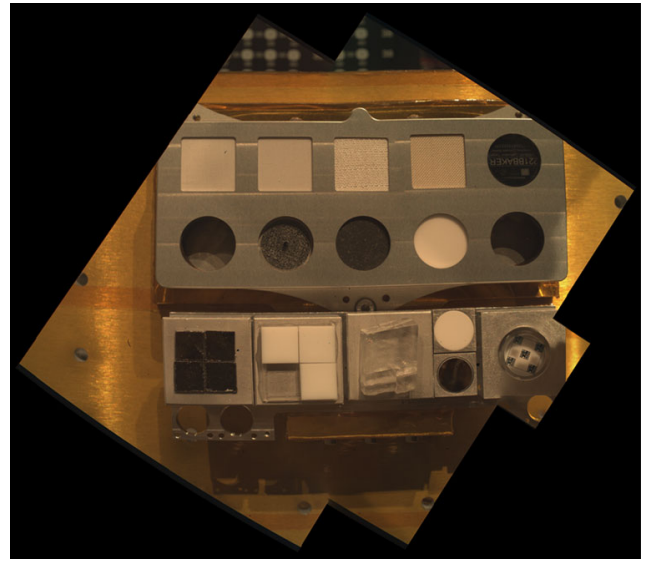

Fig. 52 Overlay of the flight model ACI and testbed-WATSON images acquired via the robotic arm placement during rover level system thermal tests (STT)

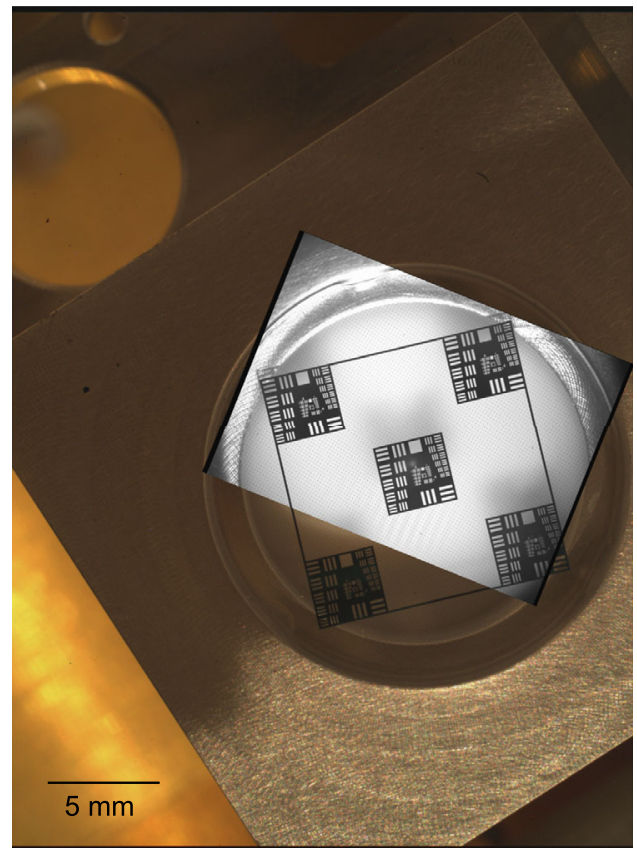

was epitaxially grown on a sapphire substrate. The resulting sample has strong photoluminescence response when excited by the deep UV laser with an emission centered at $275 \mathrm{~nm}$ and is stable over operational temperatures.

The strong response is particularly important given that the internal target is fixed to the inside surface of the dust cover and thus it cannot be placed in at the optimum laser focus. To optimize focus for internal calibration, the objective lens is retracted to the furthest position from the cover. At this point, the laser beam diameter at the $\mathrm{AlGaN}$ target is $\sim 6 \mathrm{~mm}$. The fraction of collected light is sufficient for a strong response even when the instrument is outside the nominal science performance range $\left(-80\right.$ to $\left.-20{ }^{\circ} \mathrm{C}\right)$ (Fig. 53). In addition to the $275 \mathrm{~nm}$ emission, there is a $252.9 \mathrm{~nm}$ emission from the laser that acts as a secondary 


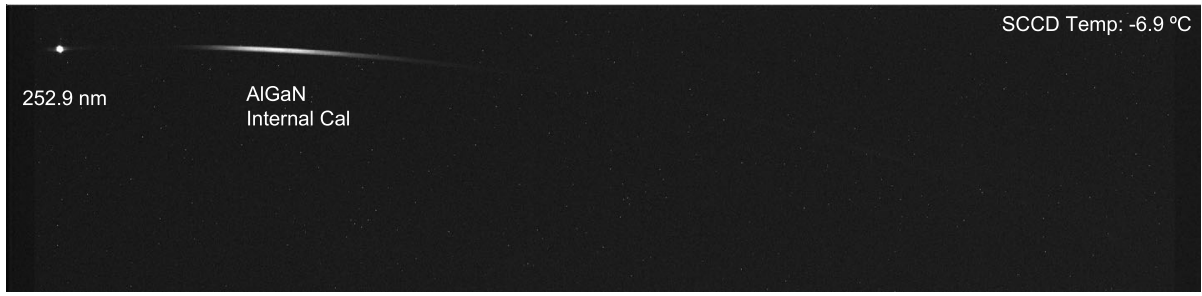

Fig. 53 The 2D image from the SCCD showing the $252.9 \mathrm{~nm}$ line and the emission from the AlGaN internal calibration target

calibration feature. With these two spectral features, the overall alignment of the instrument can be assessed. Since the $252.9 \mathrm{~nm}$ line is an atomic emission line, and it overfills the spectrometer slit, the pixel position and full-width-half-max of the line enables trending of the spectrometer alignment and focus, respectively, over temperature and instrument orientation. The $\mathrm{AlGaN}$ peak also provides a position relative to the $252.9 \mathrm{~nm}$ line to assess spectral spread and any variation due to temperature. The spectral width of the AlGaN also has the added advantage of providing an insight on SCCD rotation. In addition, in concert with the laser photodiode, the intensity of the $\mathrm{AlGaN}$ line provides insight on the overall system performance and can be used to adjust the required laser energy or number of pulses for spectral collection.

Figure 53 shows the first spectral test of SHERLOC on the rover arm acquired at Mars ambient temperature of the AlGaN internal calibration target. The SCCD temperature was slightly higher than what would typically be used for SHERLOC science on Mars. This was a result of time constraints during the test necessary to ensure that all key SHERLOC tests could be completed within schedule. Thus, the noise from this and subsequent STT tests were slightly higher than the Mars operation temperatures.

As shown in figure Fig. 53, the XY pixel position of the $252.9 \mathrm{~nm}$ line from the laser can be used to assess any laterals shifts in the spectrometer or focus change. The intensity can also be used to assess laser energy as a secondary analysis to the integrated photodiode measurement. As observed in SHERLOC TVAC, the STT data shows that the spectrometer is stable to $<1$ SCCD pixel (e.g. $<13.5 \mu \mathrm{m}$ ) over temperature and pressure. Due to data volume limitations, two dimensional SCCD images will not be commonly acquired. As such, binned spectra will provide spectral calibration (Fig. 54). From this, the FWHM for the $252.9 \mathrm{~nm}$ line is observed to be 5.4 pixels, equivalent to pre-STT results.

\subsubsection{Co-Boresighting of the Laser, ACI, and Spectrometer}

In addition to the spectrometer alignment verification, the laser to ACI to spectrometer coboresighting was assessed during STT using the BK-7 target. Since the BK-7 material has a fluorescence that is observable by the ACI and the spectrometer, an image of the focused laser spot can be located on the ACI image (Fig. 55). This was acquired by firing the laser and running ACI in video mode using a smaller image frame (light-toned square in Fig. 55). As such, in addition to testing the co-boresighting, video commanding and independent and simultaneous operation of the DEA and C\&HD were successfully tested. The results show that the laser to ACI offset did not change from instrument level tests and was located $60 \mathrm{x}$ $500 \mu \mathrm{m}$ from the center of the ACI image.

The resulting map was fit onto the ACI image to assess the boresight of the spectrometer/laser relative to the imager (Fig. 56). This image/map overlay, coupled to the laser 
SrlcSpecCalSohRaw_0625433185-28657

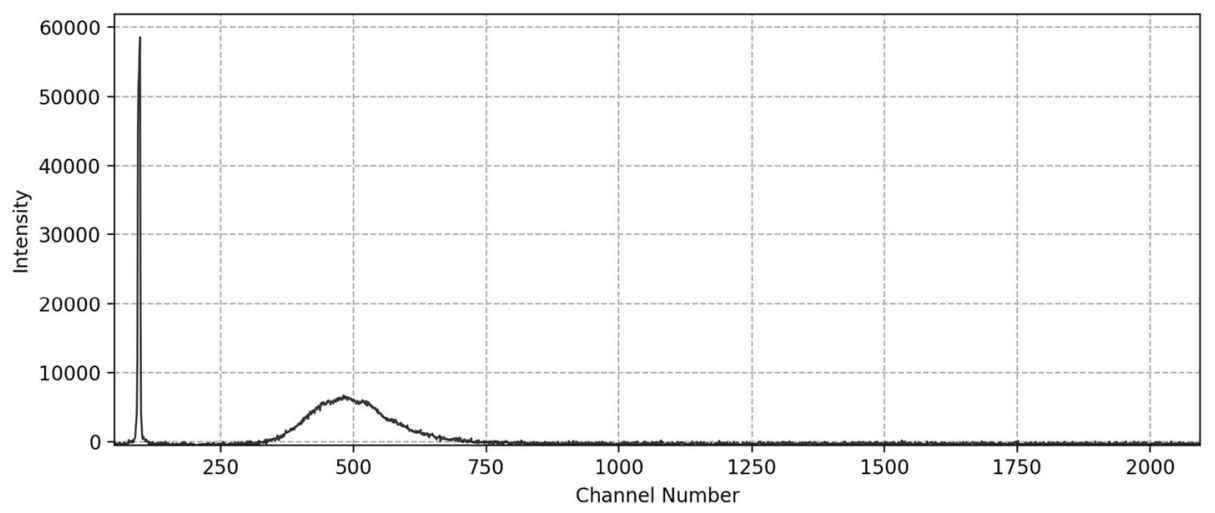

Fig. 54 2D spectrum of the AlGaN internal calibration target acquired during STT

Fig. 55 Flight unit ACI context image, inset with an ACI video subframe (light-toned square), showing fluorescence induced by laser illumination (white circle) of the BK-7 target during STT

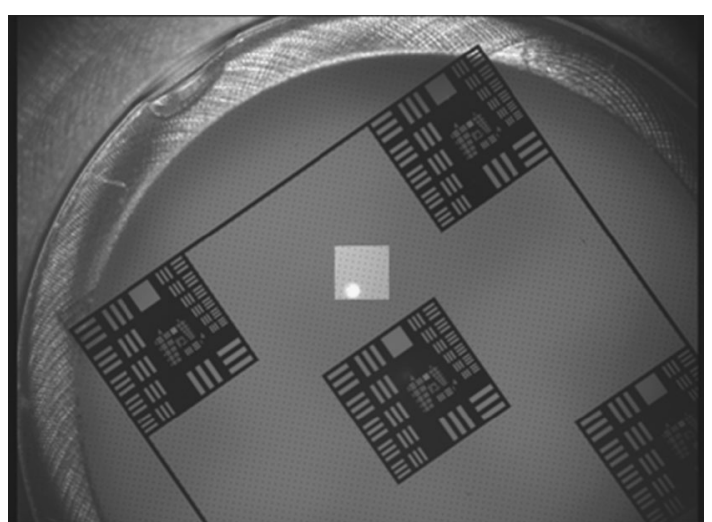

location image in Fig. 55, shows that the spectral map can be correlated with the ACI image and that there is an offset as would be expected from the laser/ACI correlation. This shows that the ACI, laser, and spectrometer paths remain co-boresighted over temperature and are unaffected by being attached to the vibration isolators, turret, and robotic arm. There is also evidence from the data that the arm stability/drift over these two measurements is within $50 \mu \mathrm{m}(<1 / 2$ a laser beam diameter $)$.

\subsubsection{Spectrometer/ACI Axial Alignment}

After establishing that the three optical paths are co-boresighted and stable over temperature under Mars environment conditions, we assessed the axial alignment of the spectrometer the ACI. This test used a target consisting of Highly Oriented Pyrolytic Graphite (HOPG). After establishing the ACI focus on HOPG (Fig. 57), the objective lens was stepped through focus such that the best focus is within the through-focus range. At each $\sim 200 \mu \mathrm{m}$ focus shift, an ACI image and DUV Raman spectrum of the HOPG was acquired. These data were plotted as HOPG intensity as a function of focus motor position and compressed image file as a means to assess focus (Fig. 58). From Fig. 58, an axial offset of $\sim 720 \mu \mathrm{m}$ is observed 
Fig. 56 Flight unit ACI image overlain by a fluorescence spectral map of the BK-7 target acquired during STT

Fig. 57 Flight model ACI image of the HOPG target at best focus, acquired during STT
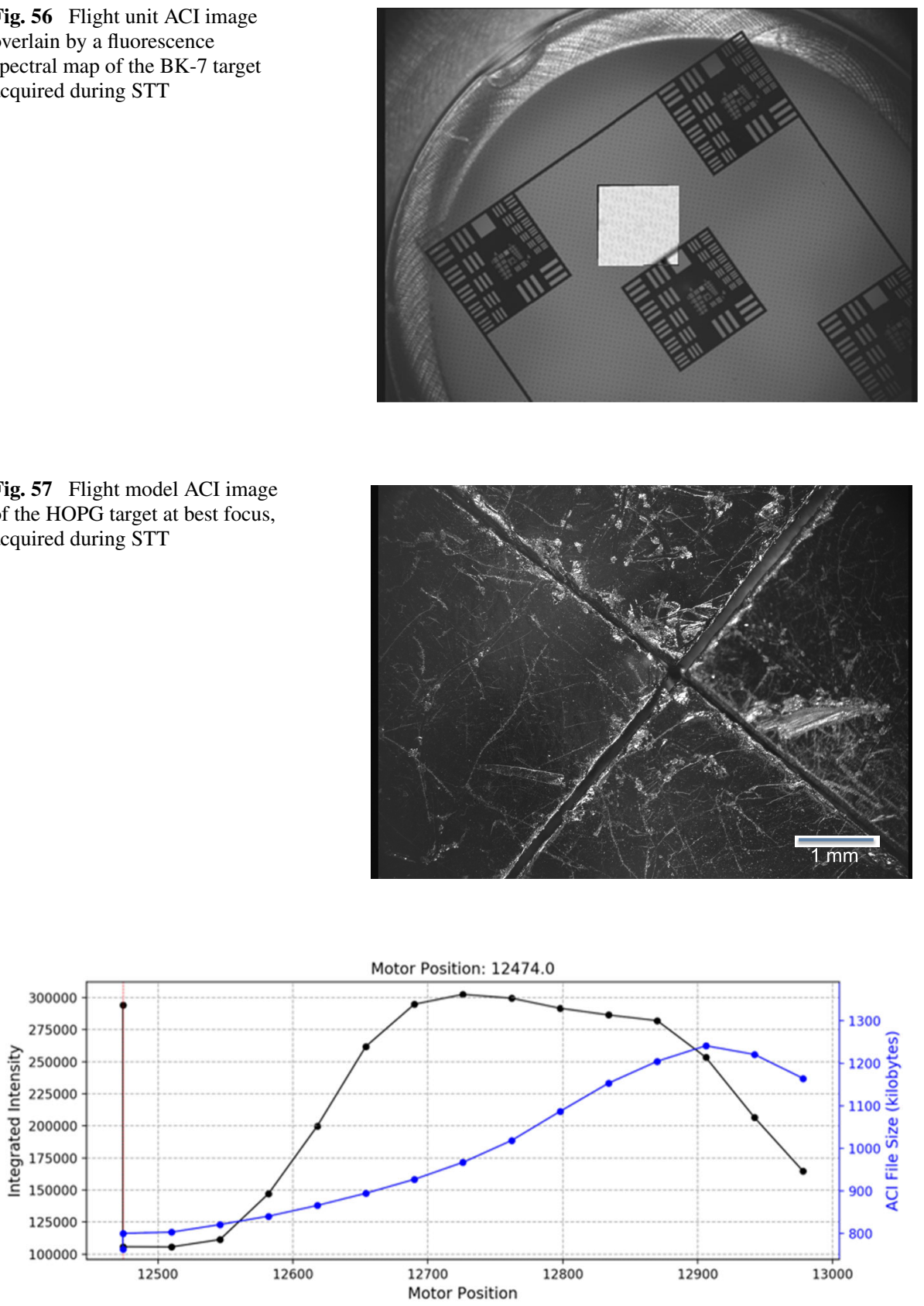

Fig. 58 A through focus plot of the HOPG Raman intensity versus ACI focus motor count (i.e., compressed file size). One Motor count position $=\sim 6 \mu \mathrm{m}$ of movement

between the ACI and the spectrometer focus. In addition, this shows the spectrometer depth of field is $\pm 600 \mu \mathrm{m}$. 

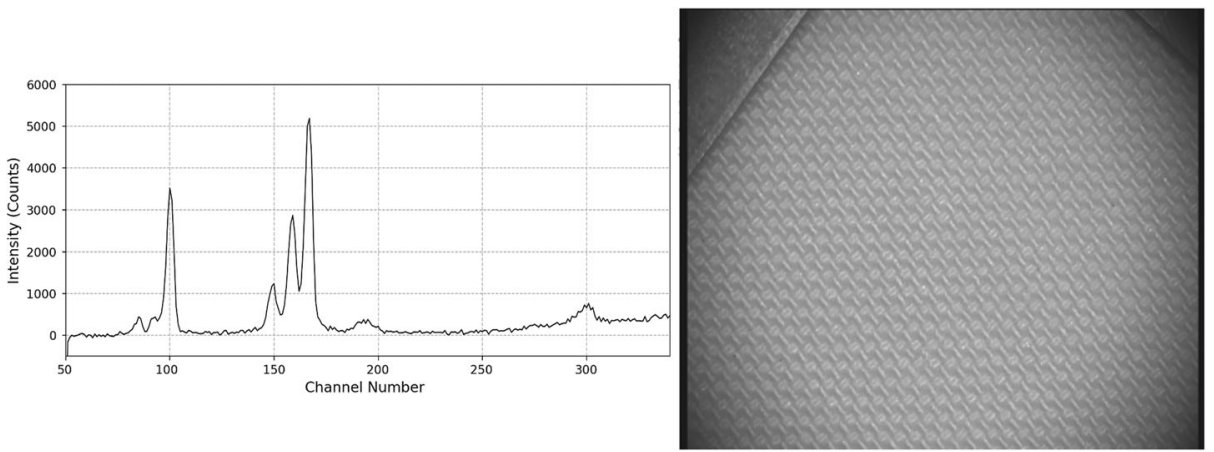

Fig. 59 The SHERLOC Raman spectrum of Teflon (left) and the flight unit ACI image (right) of the Teflon fabric on the EM SCT. For reference, the four strong peaks from left to right are: $729 \mathrm{~cm}^{-1}$ (symmetric stretch F-C-F), $1215 \mathrm{~cm}^{-1}$ (antisymmetric F-C-F stretching modes) $1295 \mathrm{~cm}^{-1}$ and $1379 \mathrm{~cm}^{-1}$ (stretching modes of C-C)

Fig. 60 Flight unit ACI image of the polycarbonate visor material and the geocache coin on the EM SCT during STT operations

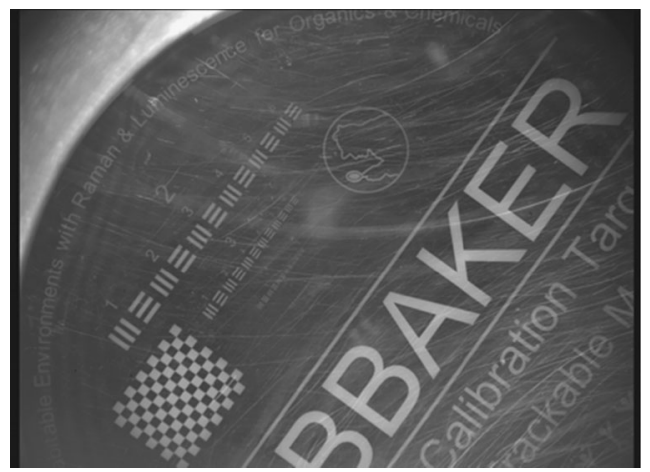

\subsubsection{Spacesuit Material Targets}

STT also enabled the first SHERLOC analysis of some of the spacesuit materials including the polycarbonate/geocache coin and the soft-goods spacesuit materials. For STT, the flight calibration target that contains these materials was covered to avoid contamination. Instead, as illustrated in Fig. 49, we observed examples on the engineering model (EM) SCT. The Teflon fabric was analyzed to assess the ability to use the ACI to focus on the fabric and to observe the well calibrated Teflon Raman response (Fig. 59). The polycarbonate and the Geocache target were imaged to determine where the ACI would focus given that the $3 \mathrm{~mm}$ thick polycarbonate visor material is above the Geocache target. Even with the fine scratches on the EM polycarbonate, the Geocache was well resolved by the ACI (Fig. 60).

\section{Mission Operations}

\subsection{Nominal Operations Description}

The SHERLOC investigation was designed to contribute to the strategic goal of understanding the history of seeking potential biosignatures on Mars. The Mars 2020 mission will make 
Fig. 61 Overview of SHERLOC operations staffing including tactical roles

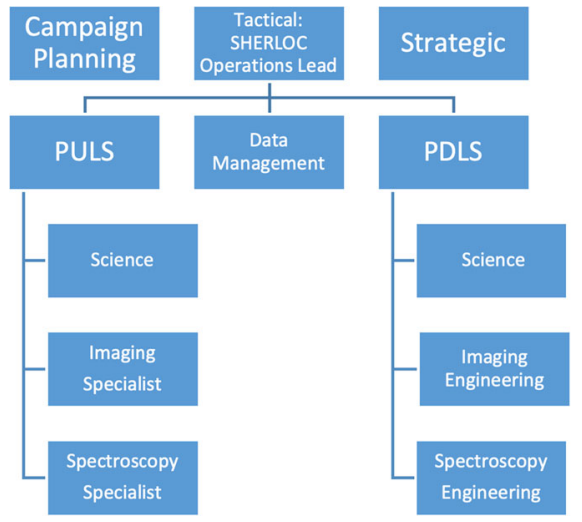

scientific measurements to better understand the history of the sites we are investigating and to determine which core samples should be extracted and cached for eventual return to Earth (Williford et al. 2018; Farley et al. 2020). This process requires both strategic planning over large scale regions of interest (hundreds of square meters) and tactical decisions that need to be planned every sol of operation for the robotic arm workspace directly in front of the rover.

SHERLOC operations are designed to be simple yet flexible to maximize the team's ability to respond to the tactical needs of the science investigation. SHERLOC activity types have been kept to a minimum, but have tunable parameters that make them highly flexible, so that they can accommodate a wide range of desired measurements and observations. For instance, spectroscopy measurements feature the ability to spatially bin acquired spectra to allow an averaged subset of data to be returned on the tactical timeline so that the team can quickly make decisions as to whether or not further measurements are needed or whether a core sample may be desired at the current location. Customizable spectral binning also allows the most desired regions of interest for a given target to be automatically analyzed in more detail so that data can be returned in the same sol without an additional ground-inthe-loop sol. In addition, WATSON imaging operations is based on many years of MAHLI heritage which has refined its operational protocols for optimal efficiency (e.g., Yingst et al. 2016).

It should be noted that SHERLOC has a unique command structure, in which the imaging sub-systems (ACI and WATSON) are handled by the DEA and the spectroscopy subsystems are controlled by the $\mathrm{C} \& \mathrm{DH}$. While working closely together, the imaging and spectroscopy side of operations are different and are thus discussed separately throughout this section where appropriate.

The SHERLOC operations staff will be deployed in both SHERLOC-specific and Mars 2020 Project-defined operational roles, enabling the team to provide substantial input toward achieving the scientific goals of the SHERLOC investigation specifically and the Mars 2020 mission in general. Daily operations require a diverse spectrum of scientific expertise including biosignature detection, astrobiology, geochemistry, mineralogy, petrology, and stratigraphy. Engineering support requires detailed knowledge of the operational abilities and safety considerations of SHERLOC, including pointing capabilities, collision avoidance, and use of the instrument's laser consumable. Specific roles include (Fig. 61):

1. SHERLOC Operations Lead (SOL).

2. Science operations Payload Uplink and Downlink Lead (Sc-PUL \& Sc-PDL). 
Fig. 62 A cropped simulated WATSON image showing the footprint of a $7 \times 7 \mathrm{~mm}$ survey scan in violet and 3 potential $1 \times 1$ $\mathrm{mm}$ detailed scans in black/gray. Detailed scans would be auto-selected, via on board data processing, based on spectral binning and ranking criteria selected by the Sc-PUL and Sp-PUL. Also shown is the spatially correlated $12 \times 16 \mathrm{~mm}$ ACI image in dotted yellow, total arm placement uncertainty in light blue, and the diameter of the abrasion area in red. Exact orientation of ACI image with respect to WATSON image is dependent on arm poses required to obtain images. All to scale

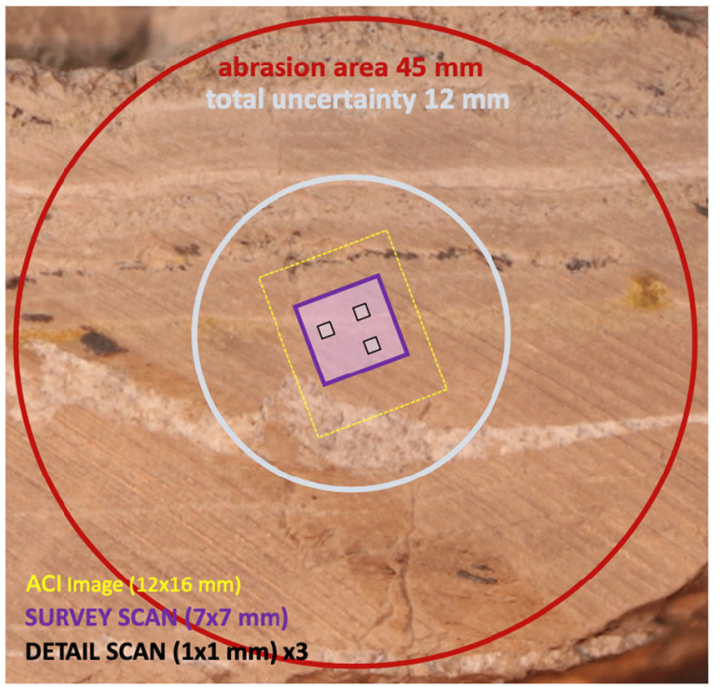

3. Imaging operations Payload Uplink and Downlink Lead (I-PUL \& I-PDL).

4. Spectroscopy operations Payload Uplink and Downlink Lead (Sp-PUL \& Sp-PDL).

5. Data Manager (DM)

6. Science team member participation during Tactical Operations planning.

7. Science operations leadership roles for Strategic and Campaign Implementation planning.

Roles 1-5 are SHERLOC-specific roles that are performed on the tactical timeline starting at the beginning of each sol. This entails Payload Downlink Lead (PDLs) assessing the most recent received from the SHERLOC instrument through to the Payload Uplink Lead (PULs) commanding SHERLOC for the next sol. These activities are overseen by the SOL who will act as the tactical point of contact for all operations, including PULs, PDLs, and the DM, allowing individuals staffing these roles to perform their jobs with minimal interference during the eventual 5-hour tactical timeline. The DM work effort spans both downlink and uplink and acts as the point of contact during tactical operations for managing the onboard data, data modes, and parameter settings. In addition, SHERLOC team members will staff Mars 2020 project-defined tactical roles (6) such as Documentarian, Keeper of Intent and Tactical Science Lead. Team members with previous Mars leadership experience will also staff Campaign Implementation planning and Strategic roles (7), such as Campaign Science Lead and Long-Term Planner. These latter roles will interact with Project Science leadership and the overall Mars 2020 Science Team for longer term planning of Perseverance activities.

\subsubsection{Spectroscopic Scans}

For investigations using SHERLOC spectroscopy, targets of high scientific interest are first identified by the Science Team using Perseverance's remote science instruments such as MastCam- $Z$ and SuperCam, with subsequent analyses being performed on abraded surfaces whenever possible. For SHERLOC spectroscopy, abraded surfaces are preferred because: (a) unabraded surfaces on Mars tend to have a significant amount of dust cover which may obscure spectral features; and (b) unaltered organic material is unlikely to be found in the upper few millimeters of the surface (cf. Razzell Hollis et al. 2020b; Kminek and Bada 2006; 
ten Kate et al. 2005; Stoker and Bullock 1997). The rover is equipped with an abrasion tool designed to flatten and smooth rock surfaces and remove the uppermost material of a rock target to a prearranged depth of $15 \mathrm{~mm}$ (Fig. 62). The gDRT is then employed to blow the resulting abrasion cuttings (dust) out of the abraded patch, clearing an area approximately $45 \mathrm{~mm}$ in diameter for SHERLOC and PIXL proximity science observations (Moeller et al. 2021).

There are three standard SHERLOC spectroscopy activity types that will be used for scientific investigations: Quick Scan, Single Placement, and Multi-Arm Placement. Additionally, whenever a sample core is obtained, an analysis will be performed on the resulting borehole wall to a depth of $>15 \mathrm{~mm}$ (Fig. 64). These activity types can take as little as 10 minutes or as long as 2 hours depending on the nature of the sample to be analyzed. Spectroscopy scans were designed to be relatively short in duration to limit the amount of rover resources utilized (available duration, data volume, power, etc.) if in competition with other rover activities and instruments. A shorter operation time also helps mitigate the effects of thermal arm drift. Thermal drift occurs when diurnal temperature changes cause the rover arm to expand and contract over time, slowly shifting the exact position of SHERLOC over the target, and rendering spatially correlated spectral maps and ACI images difficult to interpret. In order to compensate for this, SHERLOC acquires ACI images both before and after each spectroscopy scan in order to allow the PDLs to assess and correct for any drift that may have occurred.

\subsubsection{Spectroscopic Activity Types}

A variety of spectral activity types are possible for SHERLOC operations. Here we describe four of them. A Quick Scan activity enables rapid assessment of a newly abraded surface or for when operations are constrained for time. A Single Placement activity achieves SHERLOC's investigation objectives with a single arm move. Comparatively, a Multi-Arm Placement activity enables mapping of a larger fraction of the abraded surface to assess organic and mineral variability; this would be used or targets that display stratigraphic features. Finally, the Drill Hole Analysis activity provides near sub-surface analysis to examine whether the concentration or diversity of organics are better preserved and is a surrogate analysis of the acquired core to provide documentation and in-situ analysis for support of future laboratory analysis of cores after they are received on Earth.

The SHERLOC Quick Scan activity consists of raster scanning the laser over a $7 \times 7 \mathrm{~mm}$ area, with a spacing of $200 \mu \mathrm{m}$ between points, for a total of 1296 spectra (see Fig. 62). It nominally fires $4-10$ laser pulses per point at $80 \mathrm{~Hz}$ for an integration time of $0.050-0.125 \mathrm{~s}$ per point. This provides a dynamic range best suited for detection of high yield fluorescence data. This entire survey scan takes less than 20 minutes, including initialization, internal calibration, and acquisition of corresponding spatially correlated ACI images.

The SHERLOC Single Placement activity includes a "Survey Scan", as above, during which the area with the most scientifically interesting fluorescence spectral signature will be auto-selected for a more detailed scan (Fig. 62). The Detailed Scan, centered over this area of interest, consists of rastering the laser over a $1 \times 1 \mathrm{~mm}$ area, with a spacing of $100 \mu \mathrm{m}$ between points, for a total of 100 spectra. It nominally fires 200-900 laser pulses per point for detection of both low and high yield Raman and fluorescence data. Up to 3 detailed scans can be acquired per survey scan, taking 1-2 hours total duration, and includes the acquisition of multiple ACI images for the construction of high quality spatially resolved spectral maps. As mentioned previously the limitations to how long Raman spectra can be obtained is a combination of rover resources (power, data volume) and how long the PCM can keep the SCCD below $-20 \mathrm{C}$. 
Fig. 63 A simulated WATSON image with an example of a potential $6 \times 1$ mosaic that can be formed using the Multi-Arm Placement activity where the dotted boxes are simulated ACI image footprints. The thick outline scan can be done by adjusting the Table Size parameter for a $7 \times 7 \mathrm{~mm}$ survey scan down from 1296 points to 324 points. Diameter of abrasion area is in red

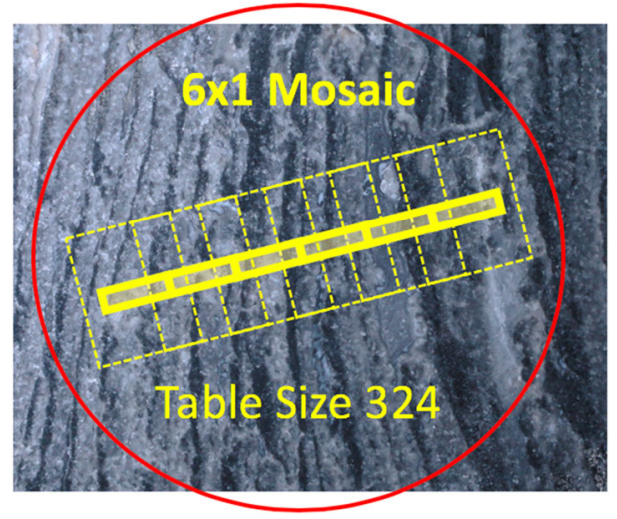

The SHERLOC Multi-Arm Placement activity is a variation of the Single Placement activity comprised of two or more Single Placements positioned to construct a mosaic of survey scans across an area of interest (Fig. 63). This activity requires multiple arm placements and the Sp-PUL must work closely with the Rover Planners (robotic arm operations engineers) to ensure proper arm placement to acquire the desired survey scan footprint. Similar to the Single Arm Placement activity, detailed scans can be added to each survey scan. A simple 2-frame survey scan mosaic could take less than 30 minutes, while a more extensive multi-frame mosaic (e.g., a $3 \times 3$, or $6 \times 1$ ), or a smaller mosaic with 1 or more detailed scans per survey scan, could take up to 2 hours to acquire.

The SHERLOC Borehole Analysis positions the SHERLOC instrument $15^{\circ}$ off a normal to the cored rock surface at a $55 \mathrm{~mm}$ standoff distance above the borehole and rasters the laser over a $2 \times 4.5 \mathrm{~mm}$ field of view down the sidewall, with a spacing of $200 \mu \mathrm{m}$ between points, for a total of 242 spectra (Fig. 64). The ACI lens is than retracted $500 \mu \mathrm{m}$, moving the center of focus to a point further up the sidewall. This activity is repeated 28 times until the swath extends to the top of the borehole, spanning a total vertical swath of $13-15 \mathrm{~mm}$, and collecting 6776 total spectra. This activity takes about 2 hours but yields 28 spectral Z-stacks and ACI context images that can be used to reconstruct the micro-stratigraphy of the upper portion of the borehole.

SHERLOC spectroscopy scans are designed for maximum flexibility, allowing the ScPUL and Sp-PUL to optimize science observations as quickly and easily as possible. To meet this goal, spectroscopy activity types have a number of tunable parameters that can be customized to fit a given observation or altered to react to resource constraints (available duration, power, etc.). Standard tunable parameters include:

Scan Table - a predefined array of points and the order in which they will be interrogated by the laser. SHERLOC can have up to 7 arrays stored in its memory at any one time, but new ones can be swapped in as needed. At the time of launch there are two general types of arrays on board SHERLOC for either Survey Scans (1296 (36x36) points) or Detailed Scans (100 (10x10) points). Depending on the spacing between points, these arrays can be as large as $7 \times 7 \mathrm{~mm}$ or as small as $1 \times 1 \mathrm{~mm}$.

Table Size - the total number of points to be interrogated within the selected Table. The number of points ranges up to the maximum number of points in the selected Scan Table. By doing a subframe of the total number of points available, a Sp-PUL could, for instance, simulate a line scan by only selecting 36 points of a survey scan (or selecting 72 points for a 'thicker' line scan, etc.). This can be especially useful if resources are 


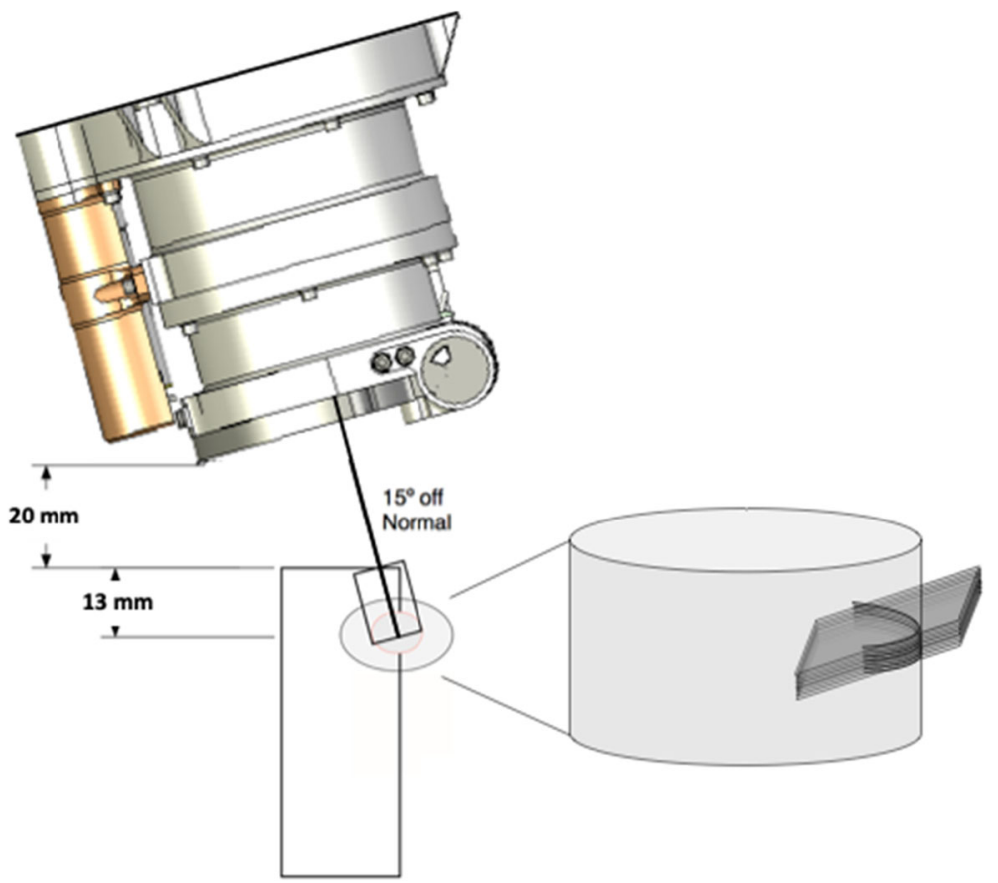

Fig. 64 Schematic of the SHERLOC spectrometer hovering over a core hole at an oblique, off normal, angle in order to perform the Borehole Analysis activity. Close up of drill hole section at right shows multiple ACI image (dark gray) acquired at sequential focus steps ( 8 of 28 total shown here)

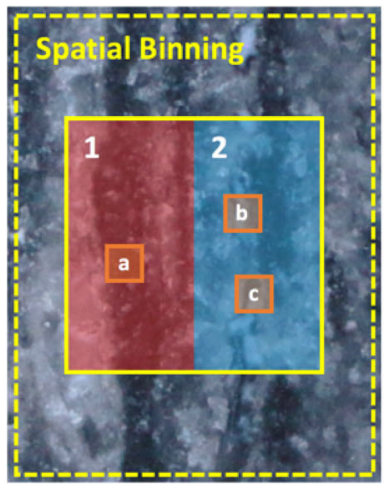

\section{Decisional data: average spectra}
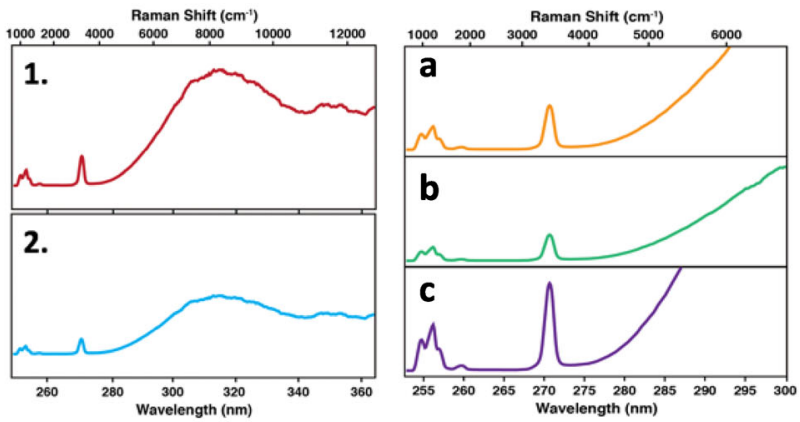

Fig. 65 Example of using the Spatial Binning Parameter on a Single Placement activity featuring a single 1296 point Survey Scan and three 100 point Detailed Scans. Spatial binning reduces the number of decisional spectra that need to be downlinked from 1596 total spectra to 5 averaged spectra, 2 for the survey scan, and 1 each for the 3 detailed scans

constrained on a given sol. For instance, a target with prominent linear features may be more effectively interrogated by using a perpendicular line scan versus a similar Survey Scan, while using a fraction of the resources (Fig. 63). 
Pulses Per Point - the number of laser pulses used to interrogate each point and acquire a spectrum (nominally at $80 \mathrm{~Hz}$ rep rate). The number of pulses per point ranges from 0 to 2000, but typically 4-10 for Survey Scans and 200-900 for Detailed Scans. A lower number of pulses is good when rapidly searching for organic fluorescence and strong Raman peaks, while a higher number of pulses is typically required for organic speciation and to detect weaker Raman peaks. In addition to laser current, pulse width, and frequency of the laser, this parameter offers a means to mitigate laser aging over the mission. Given the laser performance at the beginning of life, we do not expect that the nominal values will increase significantly.

Spatial Binning - reduces the downlinked data volume by averaging regions of the scan, thus ensuring decisional data can be downlinked in time for tactical planning (Fig. 65). Survey scans (1296 points) can be reduced down to 2, 4, 16, or 36 spectra, while detail scans (100 points) can be reduced to 1 or 4 spectra. If no spatial binning is applied to a scan, SHERLOC will downlink 1 spectrum for each point interrogated and the resultant data volume may be too high to be downlinked in time for tactical planning. As such this algorithm allows for reduced "compressed" dataset that loses spatial information but retains spectral resolution and increases SNR by approximately the square-root of the number of spectra binned.

Spectral Binning - is used to determine the number of Detailed Scans to be performed and selecting and ranking the criteria to be used for the auto-selection of these regions of interest. Up to five spectral regions can be defined and weighted for this process. So, for instance, the Sc-PUL and Sp-PUL may choose to have Detailed Scans performed on regions within the Survey Scan that exhibit the strongest fluorescence signature in the spectral range corresponding to potential aromatic molecules.

\subsubsection{Idealized Sol Path}

A Quick Scan would typically be performed immediately after an abrasion with gDRT dust clearing without ground in the loop on the first sol of a proximity science campaign. The data returned would then be used to decide how to proceed on subsequent sols, what features are scientifically interesting enough to interrogate with a Single or Multi-Arm Placement, and how to set parameters to optimize science return. For instance, linear features such as veins or laminations may best be examined using a Multi-Arm Placement mosaic set roughly perpendicular to the features of interest (e.g., see Fig. 63). Furthermore, preliminary spectra may inform which spectral regions should be selected for detailed scans, so that desired bands can be ranked higher than other spectral bands that may have a strong spectral response, but are of less scientific interest (e.g., selecting ranking criteria to preferentially scan regions in a concretion with a higher organic content versus regions containing an otherwise ubiquitous mineral). Finally, if the decision is made by the Science Team to collect and cache a core sample, the Borehole Analysis activity will be used to help document and characterize the uppermost few millimeters of the core by interrogating a vertical swath through the upper part of the borehole wall.

\subsubsection{Spectroscopy Scans}

The Sp-PDLs will be responsible for monitoring the health and performance of the instrument by analyzing data downlinked for a given sol. Routine trending will be done on all voltages, currents, and to track instrument performance under Mars ambient conditions. A dark current spectrum will be acquired every time spectroscopy data is obtained. This dark 
spectrum will be monitored over the course of the mission. Temperatures will be tracked as they inform us about the instrument's response to the environment. An internal photodiode monitors the energy that is generated by the laser over time. Laser output power will be compared to the voltages and current of the laser power supply to monitor laser lifetime. Reports on the health of the instrument will be generated daily by the Sp-PDL.

On the tactical timeline, spectral data and calibration products will be available prior to the next planning phase. This will typically consist of Autolook and Quicklook data products (Fig. 66). An Autolook products consists of (1) an average spectrum automatically generated by the SHERLOC Ground Data Systems (GDS) for areas interrogated by SHERLOC, and (2) a rough overlay showing the location of these areas on the corresponding ACI and WATSON images. Autolooks are produced within 5 minutes of the completion of a downlink pass and require no manual processing. Prior to tactical planning, the Sc-PDL will produce a Quicklook data product by annotating the average spectra to indicate the most likely mineral or organic species corresponding to the observed peaks for both the Raman and fluorescence spectral regions. Quicklooks are expected to be produced within 20 minutes of the completion of a downlink pass for use in tactical planning. A more detailed spectral map can also be generated that co-locates individual spectra with points on the ACI image, potentially correlating spectral features to features noted in the images (but this may not be available on the tactical timeline). A Sc-PDL will also be responsible for these nontactical science products within 24-48 hours of receipt of all data needed to produce these products for Science Team use in campaign and strategic planning. These higher-level data products will be further interpreted by the SHERLOC science team and compared with data from the testbed to establish the nature of organics/minerals detected. Where needed, tests on the SHERLOC EM and/or brassboard will be performed to determine the accuracy of the analysis.

\subsubsection{WATSON Imaging}

WATSON imaging will be commanded in a manner similar to the MSL MAHLI. Given the wide range of focus that WATSON can cover ( $1.8 \mathrm{~cm}$ to infinity), WATSON images are useful for providing high spatial resolution information regarding natural and abraded targets, context images for other observations (spectroscopic scans, abrasion, core holes), as well as imaging on a larger scale, such as landscape imaging and rover self-portraits (Fig. 23). WATSON will also be used for engineering support to inspect and document the state of rover hardware (i.e. wheels). Given its location on the turret at the end of the robotic arm, WATSON can be placed relative to targets to achieve viewing angles unobtainable by the other fixed cameras on the rover body. This includes viewing perpendicular to sedimentary bedding planes, looking straight down into a core hole or getting an angled view of all sides of the interior walls of the hole, viewing the undercarriage of the rover, and so forth. With multiple arm placements, WATSON can acquire mosaics and stereopairs from these varied angles as well. While WATSON is a re-flight of MAHLI, the turret on Perseverance is larger than that on Curiosity. Arm placement will account for risk of turret collision with local topography and that will limit reachable viewing positions.

WATSON Activities Following positioning by the rover's robotic arm, a WATSON imaging activity is performed. Nominally, this includes acquisition of an autofocus subframe at the center of the image to set focus at the center of the target of interest, followed by a full frame image to capture the entire scene. The additional option of a focus stack acquisition (Fig. 48) will be evaluated on a case-by-case basis, but is usually acquired at standoff distances of $7 \mathrm{~cm}$ or less, where the DOF is smaller and where capturing in-focus views on 


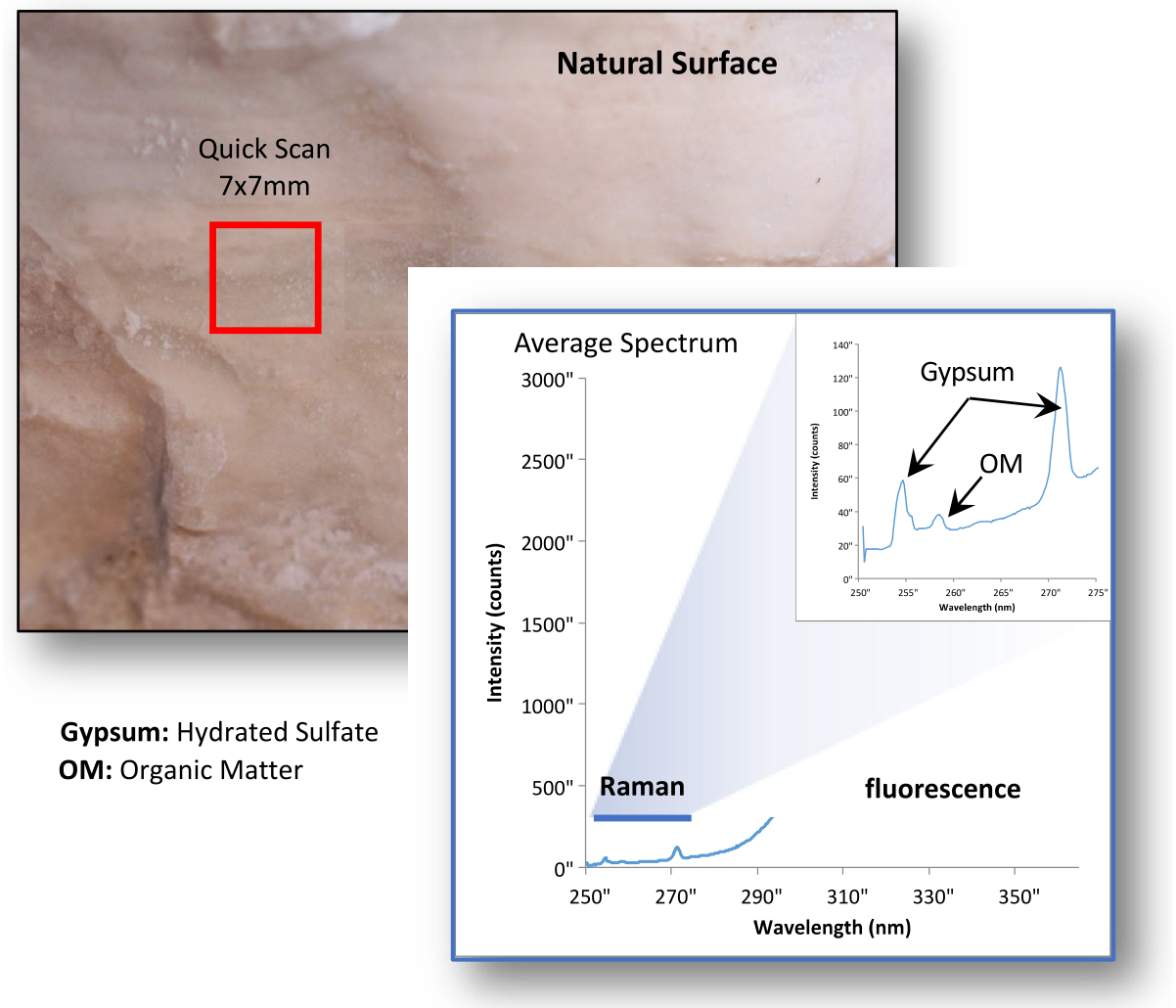

Fig. 66 An example of the data products produced by a SHERLOC Spectroscopy Quicklook product showing the location of a Quick Scan and average Raman and fluorescence spectra over a 7x7 mm area. Note an Autolook would produce essentially the same product but without the spectral annotations (i.e., gypsum and organic matter, OM)

all surface relief elements is desirable. A focus stack of up to 8 images can later be merged onboard with the WATSON Merge activity.

Tactically, the I-PUL sets image compression and downlink priority for each image to increase the likelihood of returning products to Earth that are needed to help plan the next sol's activities, based on predictions of available downlink data volume to be relayed through Mars-orbiting spacecraft. Image size can also be varied with a sub-framing approach, if desired. Different image exposure options can be set within the image command, although, most often, an autoexposure command is used. Autofocus will be used the majority of the time and will be determined over an appropriate focus range for the standoff distance from the target. Manual focus may be used when returning to a previously-used imaging position in order to speed up the observation. White light and UV LEDs may be turned on to illuminate the scene for imaging, although they will usually only be used for images obtained at night (Edgett et al. 2015).

Like the rest of SHERLOC, WATSON is on the turret at the end of the arm, and thus requires interaction with the Rover Planners (who operate the arm) for each placement to ensure the desired imaging is achieved. This provides a powerful combination of arm place- 


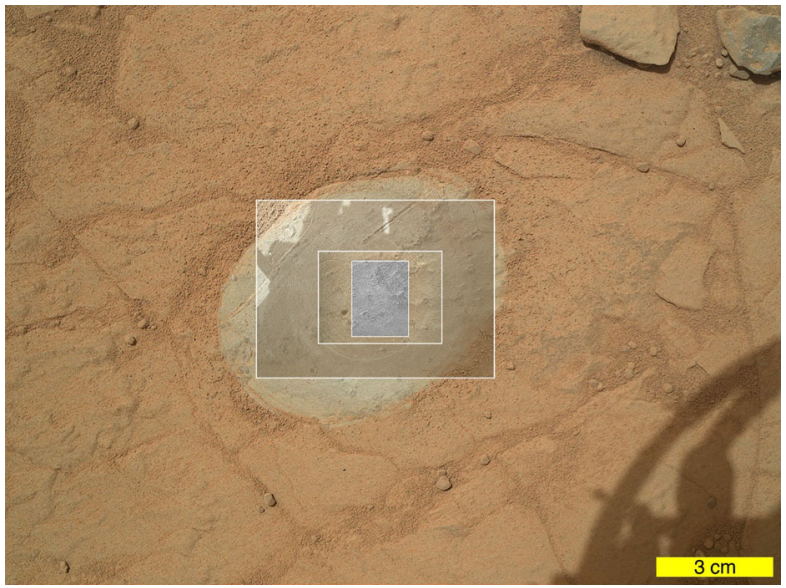

Fig. 67 Example series of nested ACI and WATSON images at typical working distances anticipated for the Perseverance mission: a $10.1 \mu \mathrm{m} /$ pixel grayscale ACI image, nested within a $2.7 \mathrm{~cm}$ working distance WATSON image, nested within a $6.8 \mathrm{~cm}$ working distance WATSON image, nested within a $26.4 \mathrm{~cm}$ WATSON context image. This nested suite was simulated using MSL MAHLI images from Sol 150 of a mudstone target named Ekwir-1. The nested position of the ACI may be rotated differently than shown here, relative to the WATSON data, unless the turret is positioned in a manner that accounts for the natural $20^{\circ}$ rotation of the different CCD's (example in Fig. 52)

ment and imaging options when used with Rover Planner and I-PUL expertise. The following types of observations can be achieved:

Stereo Imaging - a stereopair can be acquired when two different arm positions are used that provide $80 \%$ or more overlap between images. Based on MSL MAHLI experience, a lateral translation between images is normally used.

Nested Imaging - as standard practice for a target, a nested suite of images is acquired at decreasing standoff distance from the surface, centered over the target of interest in the workspace. This includes a context view from $\sim 27 \mathrm{~cm}$ standoff, a stereopair from $\sim 7 \mathrm{~cm}$, and a high spatial resolution close approach image from 3-4 cm, depending on collision risks with the surface at those close standoff distances. (Fig. 67) Nested images allow the science data analyst to extend what is observed in a small area to a larger scene that is readily placed within the context of Mastcam-Z images of the rover workspace.

QRM Imaging - a Quantitative Relief Model (QRM) provides sub-millimeter threedimensional textural information of a surface using Structure from Motion (SfM) analysis (Garvin et al. 2017). The input images for these products are generated by acquiring a small mosaic of WATSON images from the same standoff distance such that considerable overlap between images is achieved. Nominally, Garvin et al. (2017) found with MAHLI that acquisition of five arranged in a cruciform pattern centered on the target at $\sim 7 \mathrm{~cm}$ standoff provides the appropriate trade-off between QRM data product quality and downlink data volumes.

Mosaics - mosaics of any size and shape can be acquired of an area of interest, with proper planning with the Rover Planners. A consistent standoff distance is usually used, chosen with a balance between the spatial resolution desired and projected rover resources availability (e.g., activity duration, data volume available). For a given area, a mosaic from a higher standoff distance will require fewer images compared to a closer standoff distance with more frames, but the higher standoff images will be of lower res- 
olution. By default, $\sim 20 \%$ overlap between images is used between mosaic frames, but this can be varied to provide more or less stereo/overlapping coverage. With greater overlap, SfM algorithms can be used to construct a three-dimensional view of a mosaicked rock outcrop (e.g., Caravaca et al. 2020). Individual images in a mosaic are returned to Earth separately and then are mosaicked using software on the ground.

Documentation Imaging - WATSON will provide a context view for each target observed by the SHERLOC spectrometer and/or PIXL, whether or not additional imaging is done for other science. WATSON will also provide documentation imaging for engineering interactions with the surface, such as abrasion and sample coring.

Hardware Inspections - from its unique position on the end of the arm, WATSON can be placed in proximity to other parts of the rover to perform routine and anomalyassistance inspections of the rover and of other instruments. This may include routine monitoring for dust accumulation over time, calibration target imaging, wheel inspections, rover self-portraits, views to assist helicopter deployment, and arm thermal drift characterization, among others.

Target Refinement - in many cases, a WATSON image that is received on Earth can be used to refine robotic arm placement of other tools and instruments (SHERLOC spectrometer, PIXL, abrader, corer) for future activities. Such WATSON images supplement lower spatial resolution stereopair products created using the rover's engineering cameras (Maki et al. 2020). With the extra help from WATSON, precision placement of other turret instruments can be achieved for very specific targets, which is desired for detailed scans and/or sample acquisition.

Imaging Downlink The I-PDL will monitor and report on the health and safety status of WATSON and ACI on the tactical timeline. This includes monitoring temperatures to make sure the cameras are operating within nominal ranges, reviewing motor counts to check for anomalous skipping, confirming that returned focus values match expected standoff distances, making sure all expected data were received, and reviewing all data products to verify nominal performance. Conditions will be monitored as background Martian conditions change with season throughout the year. Reports on the health of the instrument will be generated daily by the I-PDL.

The DM will be responsible, daily, for keeping a record of ACI and WATSON image products onboard the rover, including knowledge of which images are stored in rover memory versus those stored in the ACI/WATSON DEA. The DM also monitors free storage space on the DEA. When the DEA begins to fill up, the DM oversees deletion of products, first ensuring that all desired images have been downlinked to Earth. As long as a given image resides in the DEA, it can also be commanded to be returned to Earth at different compressions than those originally used; the DM also oversees this process.

\subsection{Commanding}

SHERLOC operations and software heavily leverage the inherited ChemCam and MAHLI architectures that were extremely successful on the Curiosity rover. Using the rover flight software as the operations-to-instrument interface, ground-developed sequences executed on the rover will orchestrate the spectroscopy and imaging observations over LVDS command reply protocols. Figure 68 shows the high-level interface of the SHERLOC software system which consist of three main components:

(1) Ground software includes both uplink/planning support and data processing for both tactical and strategic needs; 


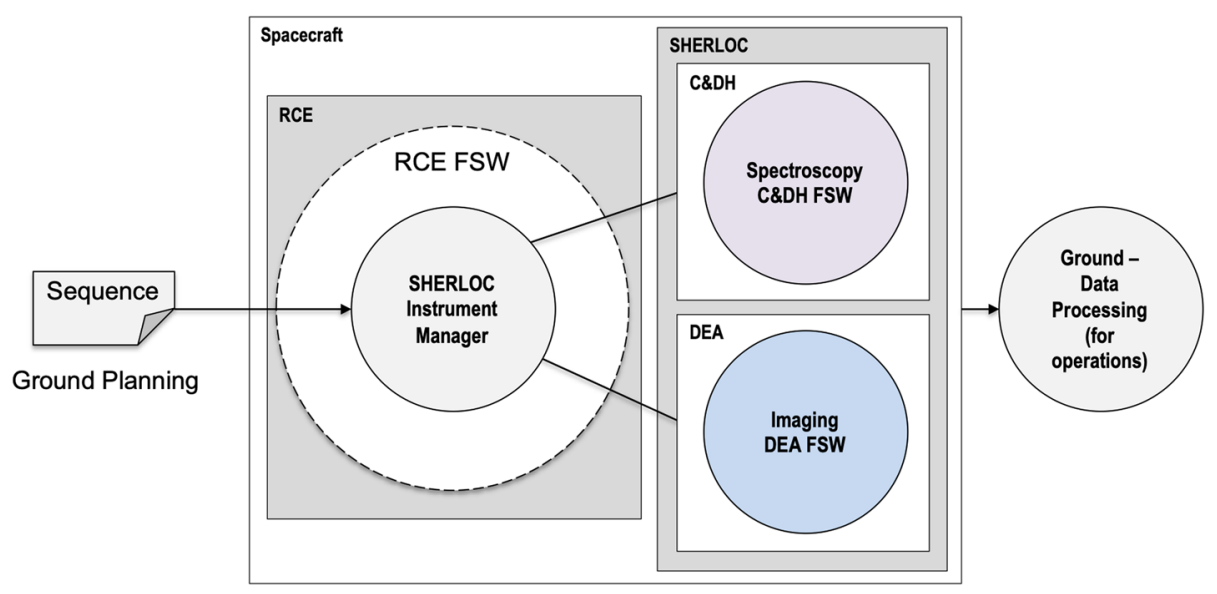

Fig. 68 SHERLOC Software System Components

(2) Rover Computing Elements Flight Software (RCE FSW) includes the interfacing software for ground operations and the instrument; and

(3) SHERLOC instrument software includes both the spectroscopy (C\&DH) and imaging (DEA).

\subsubsection{Spectroscopy and ACI}

SHERLOC spectroscopy requires coordination of both the DEA control of the ACI imager and $\mathrm{C} \& \mathrm{DH}$ control of the laser, scanner and spectrometer.

A nominal SHERLOC operation as described in Sect. 10. To execute this observation in command space, operators develop a sequence that interlaces thermal, spectroscopy and imaging activities within the design constraints of the instrument.

Before SHERLOC can be powered on, the hardware must be within allowable flight temperatures as described in the warm up Sect. 7.2. This is executed as part of the master sequence on a given sol via spacecraft command to the thermal system to turn the SHERLOC warm-up heaters into auto-mode within a parameterized control band, nominally $-20^{\circ}$ to $-25{ }^{\circ} \mathrm{C}$. The adjustable parameters are held in rover NVM. Once within flight temperatures, the main SHERLOC sequence would be executed. SHERLOC instrument would be powered on via spacecraft command that turns on the direct power switch to both imaging and spectroscopy, initializes hardware and software into standby states, and sets the instrument clock. SHERLOC is also powered off by command.

Conditioning and Calibration The first activity executed by SHERLOC after powering on is conditioning and calibration of the spectroscopy and imaging systems. The imager will acquire a series of dark images to clear the detector and assess bad or hot pixels while the dust cover is closed. IF SHERLOC has been non-operational for more than 3 months, laser conditioning is required and up to 1000 pulses will be fired into the closed ACI cover to ensure optimal performance for science is achieved. Once conditioning is complete, the instrument is commanded into the internal calibration target position, and spectra are collected using baseline laser configuration conditions. 
Table 9 Key parameters for operational efficiency

SHERLOC Key Parameters for operational efficiency

\begin{tabular}{|c|c|c|c|}
\hline Spectroscopy & Parameters & Range & Description \\
\hline Spectral Signal & $\begin{array}{l}\text { Number pulses } \\
\text { Current } \\
\text { Pulse width }\end{array}$ & $\begin{array}{l}1-2000 \\
15 / 20 / 25 / 30 \mathrm{~A} \\
20 / 40 \text { usec }\end{array}$ & $\begin{array}{l}\text { Varying the laser current and pulse width allows for } \\
\text { adjustment of laser output energy }\end{array}$ \\
\hline Spectral Noise & $\begin{array}{l}\text { Mode } \\
\text { Laser frequency } \\
\text { Regions } \\
\text { Gain }\end{array}$ & $\begin{array}{l}\text { 1D/2D } \\
10-160 \mathrm{hz} \\
1-3 \text { regions } \\
\text { Low/high }\end{array}$ & $\begin{array}{l}\text { SCCD regions can be parameterized for vertical } \\
\text { binning of the array; each region can have a separate } \\
\text { gain setting }\end{array}$ \\
\hline Scanner Motion & $\begin{array}{l}\text { Manual } \\
\text { Table } \\
\text { Ranked position }\end{array}$ & $\begin{array}{l}+/-7 \mathrm{~mm} \\
7 \mathrm{NVM} \text { tables } \\
\text { with } 100-1296 \\
\text { points }\end{array}$ & $\begin{array}{l}\text { Default tables are stored in NVM and additional } \\
\text { tables can be loaded into VM for specific } \\
\text { observations with table loading capabilities }\end{array}$ \\
\hline
\end{tabular}

Autofocus and Imaging The ACI cover is then opened and the robotic arm is commanded to move the STA into the final position for proximity science, with the ACI front window $\sim 48 \mathrm{~mm}$ above the Martian surface. The ACI is then configured to autofocus on the surface of Mars. This is completed by a single spacecraft command with parameterizable behaviors for autofocus, auto-exposure, sub-framing, and image acquisition. Autofocus for SHERLOC on abraded surfaces will be nominally completed over the full range of motion $(16.7 \mathrm{~mm})$ with 31 image samples on a sub-framed image to avoid focusing on the edges of the abraded surface. Nominally, the first image will be collected with auto-exposure and remaining images will use that exposure for all remaining images for both consistency and optimization of execution time.

Spectroscopic Parameters Due to boresight offsets between the imager and spectroscopy system, the autofocus mechanism is adjusted once in focus to the nominal spectral position ( $\sim 700 \mu \mathrm{m}$ offset). At this point the science team has complete flexibility in operating the spectroscopy system and details of key adjustable parameters are shown in Table 9.

SHERLOC spectroscopy can be commanded in a number of spectral modes including single point, table point scans, or table area scans. Table area scans have been implemented in the instrument in the event read noise was the dominate source of noise (particularly at cold temperatures) where the scanner can be rastered over an area with the SCCD integration open to create a macro pixel many times the diameter of the laser spot. Nominal operations will use the table point mode, and the SHERLOC flight model has low read noise at 5.8 e- rms. Seven certified flight tables are stored in non-volatile memory with varying sizes, spacing and orientations. Tables, like images, can be sub-framed, and smaller regions of tables can be executed by modification of the start and length of the table position for a given command. Additionally, table loads are permitted in flight and new tables can be loaded into volatile and non-volatile memory as needed. Tables are restricted to $7 \times 7 \mathrm{~mm}$ for survey scans and $5 \times 5 \mathrm{~mm}$ for region of interest selection.

For a given spectrum, the operator can control laser output energy using the laser current and pulse width parameters. In conjunction with the number of pulses, the total energy deposited on the surface can be adjusted by many orders of magnitude to control the sensitivity and targetability of native species. 
Noise performance can also be optimized given the ambient conditions. The laser repetition rate can be controlled to minimize integration time and dark noise contribution to noise. The SCCD also can be binned vertically to up to 3 regions each with programmable gain settings to increase the dynamic range or sensitivity for a given spectrum. The SHERLOC spectrometer has a design feature on the detector, a smile shown in Fig. 20, in which the Raman region is spatially dispersed over $<10$ vertical pixels in a flat line, the fluorescence region then turns up into the smile. By selecting regions, the Raman region can be read out with fewest rows and first to reduce dark noise, and signal binned to increase signal while reducing read noise as a single read. Fluorescence regions are read out next as signal is much higher for these signatures. For diagnostics purposes, the detector can also be read out as an image if misalignment issues are identified.

Process Data Behavior Once a spectral map is collected, the data set can be processed on board the instrument for decisional data, data reduction, or for autonomous retargeting areas of interest.

Decisional data and data reduction algorithms include spatial binning, spectral binning, and photodiode summary data. The spectral data are stored in a raw buffer as part of the initial command. The processing starts with the transfer of the raw data into a process data buffer via a background subtraction or copy algorithm which reduces the raw data set by a factor of 2. Spatial binning is performed on a data set by applying a mask stored in NVM (like scanner tables) that are of equal size to the original data map. Spatial binning is limited to 648 spectra per bin so a nominal survey spectrum of 1296 can be reduced into 2 spatial bins but up to as many as 1296. Spectral binning is applied to the data set by specifying up to 6 spectral zones to bin. These zones can be arbitrary in start position and length and optimized for specific spectral signatures. Additionally, the photodiode summary data can be downlinked independent of spectra if laser diagnostics are critical for tactical planning.

Regions of interest (ROI) can be identified by autonomous retargeting from an initial survey map to key features where a high resolution or high SNR map is required. Prior to running the ROI algorithm, the data must be processed onboard to avoid spurious signals and backgrounds to confuse the algorithm. First the background subtraction is applied, followed by bad pixel removal, laser normalization and finally cosmic ray removal. For timing optimization, cosmic ray removal is only applied to the ROIs used later for ranking (Uckert et al. 2019). Once the data have been preprocessed, the ranking algorithm is commanded, setting up to 6 spectral regions and applying a weighted sum to identify the highest ranked region to retarget.

Post-ranking, the spectroscopy command as described in the previous section can be executed with updating the new table to be centered at the Nth ranked position defined by the algorithm. Again, all parameters are adjustable.

Extended Operations Types The architecture allows for images and spectroscopy to be interlaced for documentation of spectral activities whenever needed. Multiple spectral maps can be sequenced as nested tables or retargeted to one or more molecular species of interest. Larger mosaics for both ACI imaging and spectral maps can be generated by coordination with the robotic arm using their nudge behavior. The SHERLOC sequencing structure allows the science team the versatility and flexibility to operate the instrument, while the command simplicity and robustness protects the hardware. 
Table 10 In-situ calibration efforts at frequency

\begin{tabular}{|c|c|c|}
\hline SHERLOC Calibration Plan & Target/Method & Frequency \\
\hline Deep UV Laser Energy & $\begin{array}{l}\text { Internal photodiode } \\
252.9 \mathrm{~nm} \text { line }\end{array}$ & $\begin{array}{l}\text { Every pulse/spectrum } \\
\text { Prior/Post nominal ops }\end{array}$ \\
\hline Spectrometer Throughput & Internal AlGaN response & Prior/Post nominal ops \\
\hline Spectral Calibration (wavelength) & $\begin{array}{l}\text { Internal AlGaN response } \\
252.9 \mathrm{~nm} \text { line } \\
\text { 2D Internal AlGaN acq. } \\
\text { AlGaN samples on SCT } \\
\text { Teflon on SCT }\end{array}$ & $\begin{array}{l}\text { Prior/Post nominal ops } \\
\text { Prior/Post nominal ops } \\
\text { Post landing/Monthly } \\
\text { Post landing/Monthly } \\
\text { Post landing/Monthly }\end{array}$ \\
\hline SCCD Noise & $\begin{array}{l}\text { Diffusil target } \\
\text { Dark acquisitions }\end{array}$ & $\begin{array}{l}\text { Post landing/Monthly } \\
\text { Every spectrum }\end{array}$ \\
\hline SCCD Hot/Dark Pixels & 2D Dark acquisitions & Post Landing/Monthly \\
\hline End to End Throughput/Intensity cal & Intensity "maze" target (SCT) & Post landing/Monthly \\
\hline Mapping Calibration & $\begin{array}{l}\text { Intensity "maze" target (SCT) } \\
\text { SaU } 008 \text { Meteorite (SCT) }\end{array}$ & Post landing/Monthly \\
\hline Focus Calibration (ACI to Spectrometer) & Intensity "maze" target (SCT) & Post landing/Monthly \\
\hline Image Resolution (ACI and WATSON) & $\begin{array}{l}\text { Intensity "maze" target (SCT) } \\
\text { Geocache coin }\end{array}$ & $\begin{array}{l}\text { Post landing } \\
\text { Post landing }\end{array}$ \\
\hline Color Calibration & Mastcam- $Z$ calibration targets & Post landing \\
\hline Internal Contamination analysis & Dark sky with laser & Post landing/Monthly \\
\hline Image Flat Fields & WATSON/ACI images of sky & Post landing/every $\sim 180$ sols \\
\hline
\end{tabular}

\subsection{Mars in-Flight Calibration Plan}

\subsubsection{Spectrometer}

To ensure that SHERLOC is operating to its full potential there are a number of internal and external calibration and assessments of health that have been included in the instrument design and operational methodology. The aspects that have been assessed prior to launch and will be calibrated during the mission are shown in Table 10.

Deep UV Laser Energy As the laser ages, the DUV laser energy will change over the mission lifetime. It is expected that the energy will initially increase and then will follow a slow decrease based on the number of pulses expended. To tune the number of pulses that are needed for the survey, detail, area/line/point modes of operation, an internal $\mathrm{SiC}$ photodiode picks off a small fraction of the laser energy and provides an assessment of laser output as a voltage and is converted into a 10 bit value. These data are recorded for each pulse, however if data volumes are limited, then the average value is provided per spectrum. These data are used to normalize the relative spectral intensity in a map. The data are also used to trend the laser performance during a map acquisition and over operations and adjust the laser parameters. For example, as the laser ages each pulse has less power. We can increase the number of pulses for detail mode to keep sensitivities similar across the mission lifecycle. Alternatively, the laser current can be increased or operated at a higher frequency if longer integration times are not desired. 
Since the photodiode is not thermally controlled, there are some temperature dependent behaviors. Data from SHERLOC TVAC are being used to generate a temperature model for the photodiode and will be used to normalize the data returned from Mars.

Spectrometer Throughput/Spectral Calibration It is expected that over the mission life, the overall instrument throughput will change. This will likely be from contamination, either from the exceedingly low outgassing rates internal to the instrument, or the very fine dust contamination of exposed optics. SHERLOC tracks this continuously using the internal AlGaN calibration target (Fig. 12). The spectral intensity of this target is stable over temperature and it is insensitive to laser/spectrometer alignment. Therefore, the laser normalized spectral signal intensity provides an assessment of spectrometer throughput. The data are obtained before all SHERLOC spectrometer operations and therefore will be used by the operations team to adjust operation parameters to maintain detection sensitivity (examples include the number of laser pulses, laser pulse frequency, and the laser current).

In addition to providing a photoluminescence signal, the $\mathrm{AlGaN}$ acts as a reflector for the $252.9 \mathrm{~nm}$ line generated by the DUV laser. This reflection is attenuated by the laser injection filter but, given its intensity, it is easily observable on the SCCD. The $252.9 \mathrm{~nm}$ line, like the primary laser line, is exceedingly narrow, and provides an assessment of spectrometer focus, instrument line shape, and — with the AlGaN emission—-provides for high-fidelity spectral calibration.

SCCD Noise and Hot/Dark Pixels One lesson learn from ChemCam on MSL, is that the e2V detector may exhibit increased dark noise and hot/cold pixels as a function of time. To assess this, each spectral acquisition is preceded by a dark (no laser) spectral acquisition. This provides a noise value for SCCD at the current temperature. As these value change with time, operations can be modified to maintain detection sensitivity.

In addition, there will be intermittent $2 \mathrm{D}$ acquisitions to assess the presence of hot or dark pixels. This can be uploaded back to SHERLOC such that these pixels are not used in the automated analysis.

End to End Throughput/Intensity Calibration While the internal AlGaN target decouples alignment from throughput analysis, the maze target is used to analyze the full endto-end performance of SHERLOC and assess the laser/spectrometer alignment. A line scan across any portion of the maze with sub beam diameter sizes $(\sim 50 \mu \mathrm{m})$ will provide a Raman signal from the silica that will decrease/increase as the beam moves from pure silica to the chrome lines. These data can be used to generate a curve that plots the intensity as a function the fraction of the beam illuminating the silica substrate. The data on Mars will be compared to the data collected in SHERLOC TVAC testing and be used to compare performance over the mission.

Mapping Calibration While the spectral mapping and ACI imaging offsets are not expected to change, the maze target on the SCT provides a means to verify this by imaging and acquiring a 2D map of the maze target and observing whether the registration between these have changed since STT.

In addition, the $\mathrm{SaU} 008$ meteorite can be used to perform this analysis. The larger grain features in the sample can be located in the spectral maps to analyze the registration when compared to the pre-launch scan done with the laboratory instrument. 
Focus Calibration Similar to the mapping calibration, the focus offset between the ACI and the spectrometer is not expected to change. However, to verify this, the maze target can be used to focus with the ACI perform a through-focus analysis with the spectrometer, much like what was accomplished with the HOPG target during STT.

Internal Contamination Analysis To ensure that all the spectral signatures that are observed are inherent to the sample, there will be occasional operations in which the spectrometer dust cover is opened, and the instrument laser is fired into the dark night sky. Through this method, any weak spectral contamination from the optics, or signature from Mars dust contamination deposited on optics, can be assessed and removed in data processing pipeline.

\subsubsection{ACI and WATSON}

Unlike MSL's MAHLI, WATSON does not have a dedicated calibration target. This is due to the limited volume on the front of the rover after the inclusion of all the coring and sample caching hardware. The MAHLI experience was such that it should be sufficient to position the WATSON via the robotic arm to image the Mastcam-Z primary and secondary targets (Kinch et al. 2020) for color, white balance, or to cross-calibrate the instrument with the Mastcam-Z cameras. The relations between image scale, working distance, and focus motor count will be confirmed and monitored for ACI and WATSON by imaging targets on the rover of known, machined scale, such as the SCT. For WATSON, a nested suite of images (working distances of $\sim 3 \mathrm{~cm}$ to $\sim 2 \mathrm{~m}$ ) of geological material in the robotic arm workspace, plus an autofocus experiment with the camera head stowed and looking out across the Martian landscape, will serve - as on MAHLI (Edgett et al. 2015-to confirm focus performance after landing. WATSON and ACI flat field images will be obtained following the method developed for the MER MI cameras and MAHLI (Herkenhoff et al. 2006; Edgett et al. 2015), in which the camera heads are pointed skyward at an azimuth $\sim 180^{\circ}$ from the sun and $\sim 30^{\circ}$ above the horizon in daylight. Per MAHLI methods, ACI and WATSON sky flat field images will be obtained at commonly-used focus positions, dust cover open and (for WATSON) dust cover closed, at two camera orientations about $180^{\circ}$ apart, every $\sim 180$ sols.

\section{Data Processing and Products}

\subsection{Ground-Based Processing Tools and Techniques}

The instrument Science Data System (iSDS) is a ground data system comprised of tools and services to manage and process Mars 2020 instrument data generated during the cruise and surface operations phases. Raw data products generated by the rover are transmitted to Earth via Mars orbiting spacecraft and the Deep Space Network, where they are reassembled into binary science data frames. Using a series of Product Generated Executables (PGEs), the iSDS generates raw science and engineering data products (Engineering Data Records: EDRs) as well as processed data products (Reduced Data Records: RDRs) for analysis by the science team. SHERLOC data are autonomously processed and packaged for archive in the Geosciences Node of NASA's Planetary Data System for access by the broader planetary science community. Table 11 provides a list of all EDRs and RDRs that will be produced by the iSDS.

The iSDS pipeline runs autonomously as data are received from the rover, with each step executing once the input data products become available. In the event that a partial data set 
Table 11 EDR (blue) and RDR (green) products to be archived in the NASA Planetary Data System (PDS), generated by the iSDS

\begin{tabular}{|c|c|}
\hline Product & Description \\
\hline Spectroscopy Active Frame & $\begin{array}{l}\text { Raw spectral intensity values of science and calibration targets } \\
\text { collected while the laser is firing }\end{array}$ \\
\hline Spectroscopy Dark Frame & $\begin{array}{l}\text { Raw spectral intensity values of science and calibration targets } \\
\text { collected without triggering the laser }\end{array}$ \\
\hline Laser Photodiode Response & $\begin{array}{l}\text { Photodiode response values collected for each laser shot, } \\
\text { representing the laser energy deposited at the target }\end{array}$ \\
\hline Scanner Position & $\begin{array}{l}\text { Scanner azimuth and elevation angle positions at each point, } \\
\text { representing the laser scanner mirror orientation }\end{array}$ \\
\hline WATSON Image & Raw, un-corrected WATSON image \\
\hline ACI Image & Raw, un-corrected ACI image \\
\hline Processed Spectra & $\begin{array}{l}\text { Science and calibration target spectral data that has been } \\
\text { processed to remove cosmic rays, subtract dark frames, correct for } \\
\text { detector gain variability, and normalize to laser intensity. A table } \\
\text { of wavelength values associated with each CCD pixel is also } \\
\text { included in this product }\end{array}$ \\
\hline Drift-Corrected Laser Shot Locations & $\begin{array}{l}\text { Laser shot positions in the target frame and the image frame, } \\
\text { corrected for robotic arm drift }\end{array}$ \\
\hline Spectrum Intensity Maps & $\begin{array}{l}\text { Up to } 36 \text { configurable spectral intensity maps representing Raman } \\
\text { or fluorescence spectral variability }\end{array}$ \\
\hline Laser Intensity Map & $\begin{array}{l}\text { An image representing the photodiode laser intensity variability } \\
\text { within a measurement }\end{array}$ \\
\hline $\begin{array}{l}\text { Image }+ \text { Spectrum Intensity } \\
\text { Map Overlay }\end{array}$ & $\begin{array}{l}\text { A multi-channel image file containing an ACI reference image and } \\
\text { several spectral intensity maps, aligned with the context image }\end{array}$ \\
\hline Corrected WATSON Image & WATSON image geometrically and radiometrically corrected \\
\hline Corrected ACI Image & ACI image geometrically and radiometrically corrected \\
\hline
\end{tabular}

is downlinked (e.g. due to unfavorable weather at the time of downlink, or large datasets broken into multiple transmissions from Mars), updated versions of EDRs and RDRs will be generated and subsequently archived.

The iSDS pipeline is broken into several components: spectroscopy processing, image processing, engineering and state-of-health assessment, and spectral map generation. Figure 69 shows a data flow diagram for the entire iSDS pipeline, with each unit representing a single PGE processing step. The iSDS pipeline culminates in the generation of an Autolook Report - a PDF containing an overview of the processed spectra, context images, laser shot locations, instrument state of health, and spectral intensity maps. Science team members are notified upon the generation of this report to support surface operations target selection. Each processing step in the iSDS pipeline is detailed in the following subsections.

\subsubsection{Acquired and Processed Data}

These PGEs will read (binary) SHERLOC science data frames (including science, calibration, and internal calibration targets), parse the active and dark frames, laser photodiode array, and scanner position information, and output the data to EDRs. Each EDR contains multiple tables containing instrument state-of-health information (e.g. CCD temperature, laser configuration parameters, timing). The SCCD may be divided into up to five regions, each of which may be configured separately to effectively extend the dynamic range and sen- 


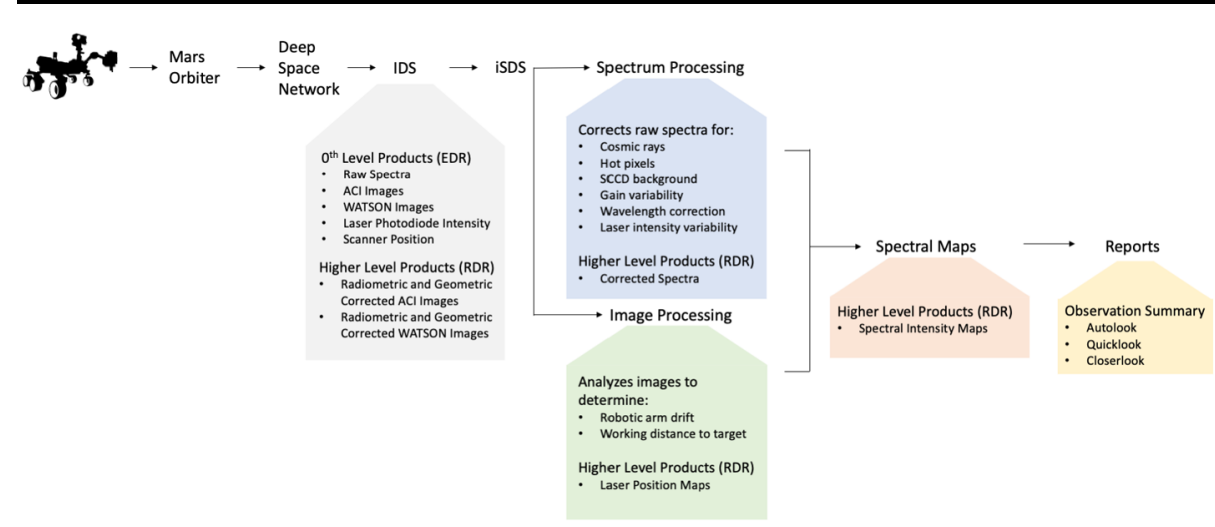

Fig. 69 A data flow diagram representing the major components of the iSDS pipeline: spectroscopy processing (blue), image processing (green), and spectral map generation (red)

sitivity of the instrument. Each SCCD region is represented as a distinct table in SHERLOC EDR and RDR files.

Data processing on the rover automatically removes cosmic rays from spectrometer data. Cosmic rays are identified in each active and dark frame and replaced with an average or interpolation between spectral intensity points outside of the cosmic ray region. Cosmic ray candidates are selected using the method described in Uckert et al. (2019) - a histogram is generated for all wavelength channels (intensity values are binned) a cosmic ray is detected when a bin lies beyond a configurable threshold value, and at least one empty bin precedes the cosmic ray candidate. The cosmic ray reduced spectrum and a list of cosmic ray candidate locations are generated as output products.

The SHERLOC dark frame is subtracted from the active frame for each SCCD region. Dark frame spectra are collected during each spectral measurement at each map position by exposing the SCCD for the same duration as an "active" exposure, but suppressing the laser trigger. Dark frame subtraction corrects for dark current and hot pixels.

The SCCD gain may be defined independently for each region to provide higher CCD sensitivity in Raman spectral regions than fluorescence spectral regions, where a higher fluorescence signal is expected. Gain correction allows for direct comparison between each spectral region.

A wavelength correction for the Raman peak locations is performed through the use of the internal AlGaN target and the SCT. Spectra from these targets are used to empirically define a polynomial relationship between SCCD channel number and wavelength. Polynomial coefficients are updated during routine calibration measurements to correct for potential instrument alignment changes. A table of wavelength values is appended to each spectral RDR product.

The intensity of the SHERLOC laser varies over the duration of a measurement primarily due to temperature fluctuations in the laser power supply. An internal photodiode analyzes some of the picked off laser energy every pulse in order to determine laser intensity. The laser photodiode response is a function of the energy deposited on the target by the laser; spectra must be normalized to the maximum laser energy to account for a potentially variable source to directly compare spectra within a measurement.

SHERLOC measurements of the internal AlGaN target are routinely collected to characterize the performance of the instrument over time. Fermi-Dirac and Gaussian functions are 
fit to the most prominent Raman features in the spectra to determine the peak characteristics of each measurement. The peak position and shape of all features in each spectrum are trended to evaluate the performance and alignment of the SHERLOC spectrometer over the lifetime of the mission.

Raw ACI and WATSON images are radiometrically and geometrically corrected. Before, after, and throughout a nominal SHERLOC spectral measurement, ACI images of the target are acquired. A feature recognition algorithm is applied to all ACI images collected as part of an observation, images are aligned, and offsets between images are calculated. Under ideal laboratory conditions, no offset would exist between images. During operations on Mars, slight offsets are expected between images due to environmental effects from wind, thermal expansion of mechanical components caused by temperature variability, and robotic arm drift. These offsets are characterized by image transformation parameters, capturing the linear offset, rotation, and varying distance to the target. These effects will be characterized during the course of the mission to monitor instrument and robotic arm performance.

Scanner orientation points (azimuth and elevation angle) at each measurement position are converted to $(\mathrm{x}, \mathrm{y})$ positions in the target plane based on the center of the laser position and the rotation of the scanner relative to the image plane.

The integrated intensity within a specified wavelength region for each point is calculated and positioned on a greyscale map based on the drift-corrected laser shot positions in the target plane. Each of the 36 wavelength ranges represents a Raman or fluorescence peak or region of interest that may be modified or configured to better suit a specific target.

Greyscale maps generated from the Map Conversion PGE are overlaid on a reference ACI context image in a multi-channel image file. These spectral intensity maps may reveal spatial variability of Raman or fluorescence features that could correspond to geologic boundaries or the distribution of organics, which may be correlated with morphologic or textural features present in the context image.

This PGE also generates drift-correct laser shot positions in the target plane (x,y) and image plane $(i, j)$ associated with a reference ACI image.

\section{Contamination Control and Planetary Protection}

The SHERLOC instrument is extremely sensitive to deposition of molecular deposits onto the optics and the calibration target. Organic species are highly absorbing in the UV with aromatic species able to generate fluorescence photons from higher energy photons. Both of these effects result in degraded signal from target samples as any generated native fluorescence from contamination is not in focus and hence would not have a major contribution to collected spectra. It is for this reason that SHERLOC's successful scientific function is dependent upon contamination mitigation. Organic species that outgas from hardware tend to be aromatic and have varying degrees of signal attenuation. Also, it is possible that solar UV causes the contamination to polymerize, resulting in changing absorption parameters during the mission. Beyond molecular contamination, it is possible that particulate deposition (both fluorescent and non-fluorescent) onto optical surfaces results in degraded signal. The contamination controls on SHERLOC were designed to limit these effects. These contamination sources may be external or internal to the SHERLOC instrument, and all were monitored during development of the SHERLOC hardware.

External contaminant sources include the rover, adjacent Mars 2020 instruments, aeroshell (jettisoned before landing), environmental conditions, or operations-generated contaminants including those in ATLO, interplanetary cruise, and Entry Descent \& Landing 
(EDL) environments. Self-contamination can include outgassing of materials present within the STA. The STA was designed to have most of the electronics boxes outside of the spectrometer bench on the back side of the STA. However, there are a few electronic devices that had to be within the optical bench and have the potential to generate outgassing in the form of organics, $\mathrm{H}_{2} \mathrm{O}$, and silicones. These species can be distributed throughout the instrument during both instrument and rover-level thermal vacuum testing, cruise, EDL, and mission operations.

Particulate contamination is primarily from particles trapped during instrument integration and test (I\&T). These particles may become dislodged during vibration events at the instrument- and rover-level (acoustic testing, launch, and EDL).

\subsection{Contamination Control}

\subsubsection{Approach}

The Contamination Control approach for SHERLOC employed a set of mitigations designed to enable the integrity of the science investigation. The following contamination mitigation methods were implemented in the design and build of the SHERLOC instrument:

- Design considerations to reduce the amount of cabling and electronics inside the optics bench within the STA.

- Careful material selection and testing of all selected materials for the STA.

a. Evaluation of transmission loss due to attenuation.

b. E595 testing of all materials to determine total outgases mass loss.

c. E1559 testing of select materials.

d. Perform UV fluorescence/Raman analysis of parent material and vapor deposited materials.

- Incorporate decontamination heaters for the optics and the calibration target to prevent deposition of molecular contamination onto contamination sensitive surfaces or to bake off deposited contaminants during cruise and on Mars.

- Incorporate Tenax getter inside the optics bench to minimize molecular contamination deposition onto optics by entrapment onto the getter.

- Utilize an ultra-low outgassing adhesive, Nusil SCV-2585, to bond optics.

- Implement a rigorous vacuum baking scheme to reduce outgassing.

- Perform sampling and analysis of contaminants present on the instrument and in ground processing environments for characteristic signatures that may be detrimental to the science investigation. This included the use of witness plates (now stored at NASA JSC) to monitor contamination levels during I\&T.

\subsubsection{Requirements}

Degradation of the optical throughput for the life of the mission is of utmost concern for the SHERLOC instrument. A major contributor to throughput loss is condensation of materials onto optics with significant absorption or fluorescence with the wavelength range of 248 to $354 \mathrm{~nm}$. To determine the effect of the contamination contribution from the Perseverance rover to the STA, condensate collected from the Curiosity rover (this material is considered analogous to expected outgassing materials from the M2020 rover) system thermal vacuum test was extracted with Dichloromethane and spin coated onto an optic. The coated optic was then evaluated using the SHERLOC laboratory prototype instrument for 
Table 12 Dichloromethane extracted constituents from the Curiosity rover thermal vacuum testing and effect to SHERLOC. Note the primary outgassing constituent from MSL was water

\begin{tabular}{llll}
\hline Chemical Function Groups & Estimated \% & Sources & Effects on SHERLOC \\
\hline Aliphatic Hydrocarbons & $35 \%$ & Hydrocarbon lubricants & Attenuation \\
Aromatic Hydrocarbons & $7 \%$ & $\begin{array}{l}\text { Epoxy bisphenol A and solvents } \\
\text { Plasticizers, ester hydrolysis } \\
\text { products }\end{array}$ & $\begin{array}{l}\text { Fluorescence/Attenuation } \\
\text { Attenuation }\end{array}$ \\
Silicone & $14 \%$ & $\begin{array}{l}\text { RTV, polydimethylsiloxane } \\
\text { oligomers }\end{array}$ & Attenuation \\
Others (all minor components) & $10 \%$ & Amides, amines, alcohols & Attenuation \\
\hline
\end{tabular}

Fig. 70 Percent transmission loss of Curiosity rover condensate

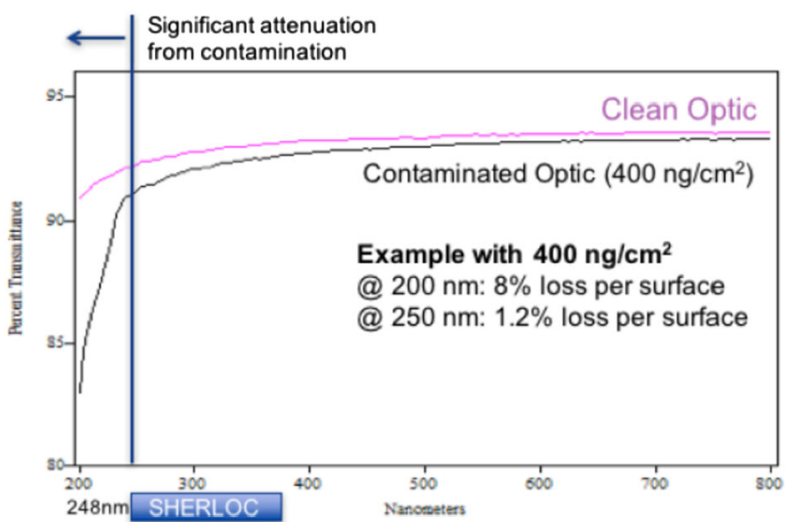

Table 13 Attenuation effect on optical path transmission determined from MSL condensate deposited onto optic

\begin{tabular}{llllll}
\hline MSL Condensate Concentration & None & $50 \mathrm{ng} / \mathrm{cm} 2$ & $100 \mathrm{ng} / \mathrm{cm} 2$ & $200 \mathrm{ng} / \mathrm{cm} 2$ & $300 \mathrm{ng} / \mathrm{cm} 2$ \\
\hline Laser Path \% T & 0.31 & 0.31 & 0.30 & 0.28 & 0.26 \\
Collection Path \% & 0.21 & 0.21 & 0.20 & 0.19 & 0.18 \\
Margin against Requirements & $28 \%$ & $24 \%$ & $20 \%$ & $13 \%$ & $3 \%$ \\
\hline
\end{tabular}

Assumptions: Laser $1 \mu \mathrm{J} /$ pulse, 900 pulses/point, Laser/Collection path start with L5 req. values (CBE is higher), Aliphatic Raman Response

fluorescence affects and transmission loss through the optic. The chemical makeup of the Dichloromethane extract with description of effects to SHERLOC is described in Table 12.

The coated optic was analyzed for \%transmission loss in the spectral range from $200 \mathrm{~nm}$ $-800 \mathrm{~nm}$ as shown in Fig. 70 using a Perkin Lambda 1050 UV-Vis-NIR spectrometer.

Analysis of the coated optic using the SHERLOC laboratory prototype instrument facilitated determination of the attenuation of the MSL surrogate condensate material through the laser path and the collection path optics of the instrument and is presented in Table 13.

Analysis was performed to ascertain the fluorescence effect to transmission loss due to the presence of fluorescence from the optics in the common path (illuminated by both the laser and observed by the spectrometer). The incorporation of a pinhole in the spectrometer 
significantly mitigates fluorescence from the optics in the common path. The fluorescence with the calculated cross sections suggests no significant effect from fluorescence and the effect of the MSL condensate is identical to attenuation analysis results. Based upon the results of the MSL condensate analysis, the SHERLOC team set an upper limit of $150 \mathrm{ng} / \mathrm{cm}^{2}$ of organic contamination on each optical surface as necessary to attain a $16 \%$ margin against meeting stated analysis capabilities on Mars.

\subsubsection{Materials Testing}

Attaining a maximum level of $150 \mathrm{ng} / \mathrm{cm}^{2}$ of organic material deposition on optical surfaces at Mars landing is not trivial. Significant efforts were made to mitigate deposition during I\&T and cruise. Considerable efforts were also made in materials testing and elimination of any materials that are highly outgassing both inside the optics bench and on the STA. Materials testing included ASTM E595 testing for \%TML (Total Mass Loss) and \%CVCM (Collected Volatile Condensable Materials) of all materials used inside the optics bench as well as materials on the STA. In addition to ASTM E595 testing, selected materials utilized inside the optics bench were further evaluated using E1559 testing to facilitate thermal vacuum bakeout and outgassing rate calculations for materials of concern during the cruise phase of the mission.

From E1559 testing of materials, specifically for the SHERLOC optics bench and E1559 testing of the M2020 rover S13GP:6N/LO-1 paint with GE SS-4044 Primer, it was determined that to meet a total accumulation of $<150 \mathrm{ng} / \mathrm{cm}^{2}$ on the optics and the calibration target, decontamination heaters would be required for the cruise phase of the mission. In particular, deposition from rover paint and outgassing materials from the rover electronics vent areas were estimated to be in excess of $150 \mathrm{ng} / \mathrm{cm}^{2}$ (possibly as high as $\sim 450 \mathrm{ng} / \mathrm{cm}^{2}$ in the upper bound) upon arrival at Mars if no additional mitigation was performed. The estimate of the organic contamination deposition to the SHERLOC Calibration Target was also determined to be higher than acceptable during cruise. Because of these analyses, decontamination heaters for the SHERLOC optics and the calibration target became part of the instrument design.

Additional materials testing included fluorescence analysis of both bulk organic materials including adhesives, paints, coatings, a connector, and, where feasible, the outgassing material vapor deposited onto a target in vacuum. Samples were prepared and deposited onto 6061 Aluminum (T6) which had previously been $\mathrm{O}_{2}$ coated. The aluminum slides were transferred in cleaned glass Petri dishes lined with aluminum foil fired at $500{ }^{\circ} \mathrm{C}$ for 12 hours. Vapor-deposited material was collected by using a modified ASTM E595 tester to heat the material to $125^{\circ} \mathrm{C}$ in vacuum and deposit directly onto prepared aluminum targets at $25^{\circ} \mathrm{C}$. The vapor-deposited material was transferred inside special clean containers to the Raman/Fluorescence laboratory instrument.

Spectra in the study was collected using a custom built, DUV-resonance Raman/Fluorescence instrument in the SHERLOC team laboratory (Bhartia et al. 2012b, 2014; Abbey et al. 2017; Sapers et al. 2019). An example of the spectrum of cured Hysol 9309 bulk material obtained from the Raman/Fluorescence instrument is shown in the Fig. 71. Fluorescence spectrum of the vapor deposited Hysol 9309 material is shown in Fig. 72. Note that the bulk material exhibits significant spectral fluorescence intensity while the vapor deposited material exhibits little fluorescence. In addition, a Raman spectrum of the vapor deposited material was also performed without any observable signal.

The materials analyses performed prior to build of the SHERLOC flight instrument were designed to provide screening of organic materials to be used in construction of the instrument for their potential to contaminate the optics. Additionally, the collected spectra provide 


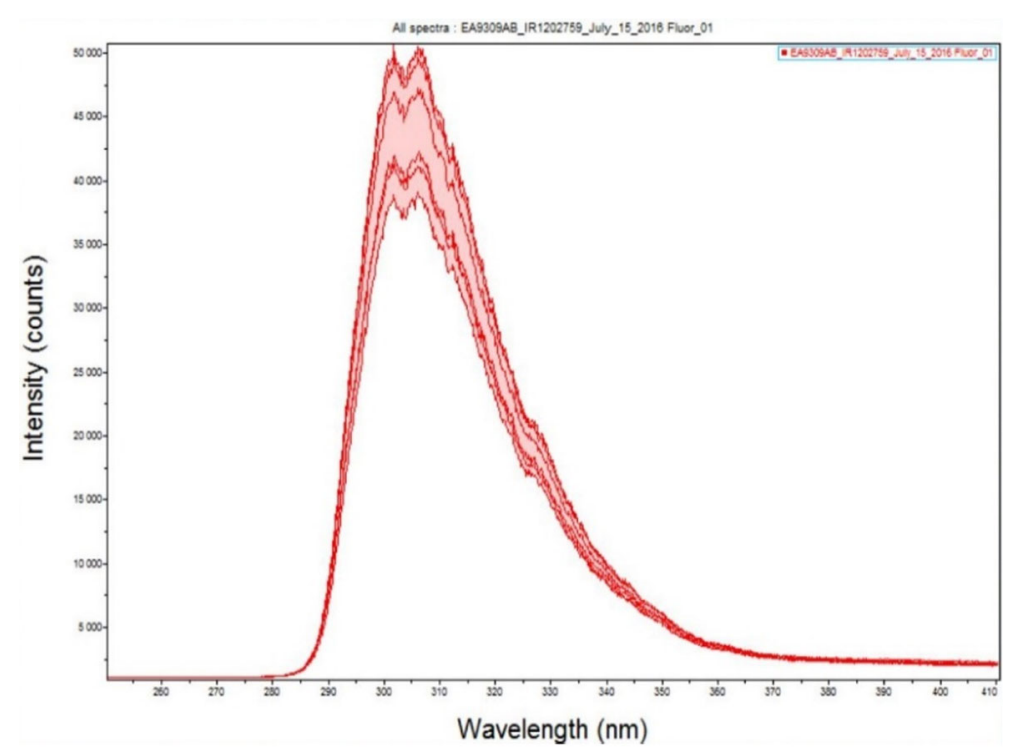

Fig. 71 Fluorescence spectrum of cured Hysol 9309 bulk material

Fig. 72 Fluorescence spectrum of Hysol 9309 vapor deposited material

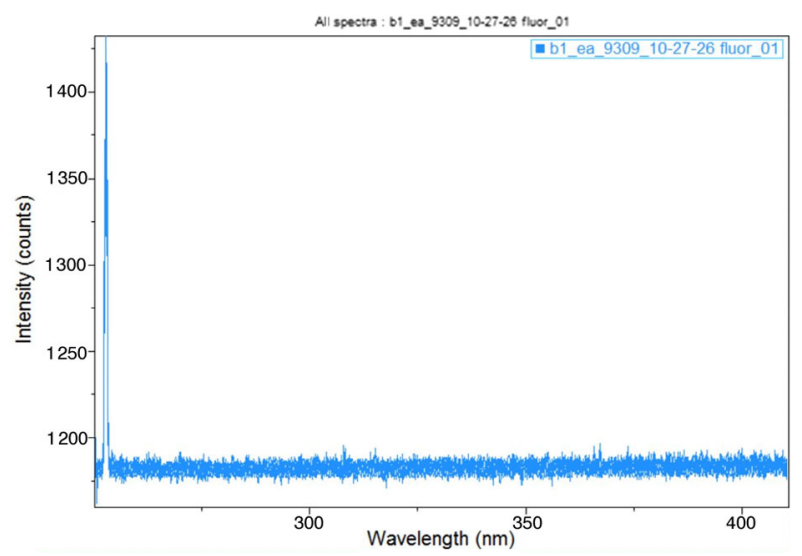

a database for all materials used inside the optics bench for future use by the instrument team.

In recognition of the contamination risk to the optics from materials outgassing inside the optics bench, a new ultralow outgassing silicone was tested and chosen for bonding the optics. This silicone, Nusil SCV-2585 Silicone, Ultralow Outgassing ${ }^{\mathrm{TM}}$ was evaluated for outgassing, mechanical strength and ease of use by the SHERLOC team. The silicone is a platinum cured system and has a 1:1 mix ratio for the catalyst and polymer. The mix ratio mitigates mixing errors and the need to meet specific humidity requirements resulting in a more robust cure outcome for the material. A sample of cured material was used for vapor deposition onto an optic for percent transmission loss in the spectral range from $200 \mathrm{~nm}-$ $2500 \mathrm{~nm}$ using a Perkin Lambda 1050 UV-Vis-NIR spectrometer. Material from the cured silicone heated to $100{ }^{\circ} \mathrm{C}$ in a vacuum was collected onto a sample quartz window cooled 


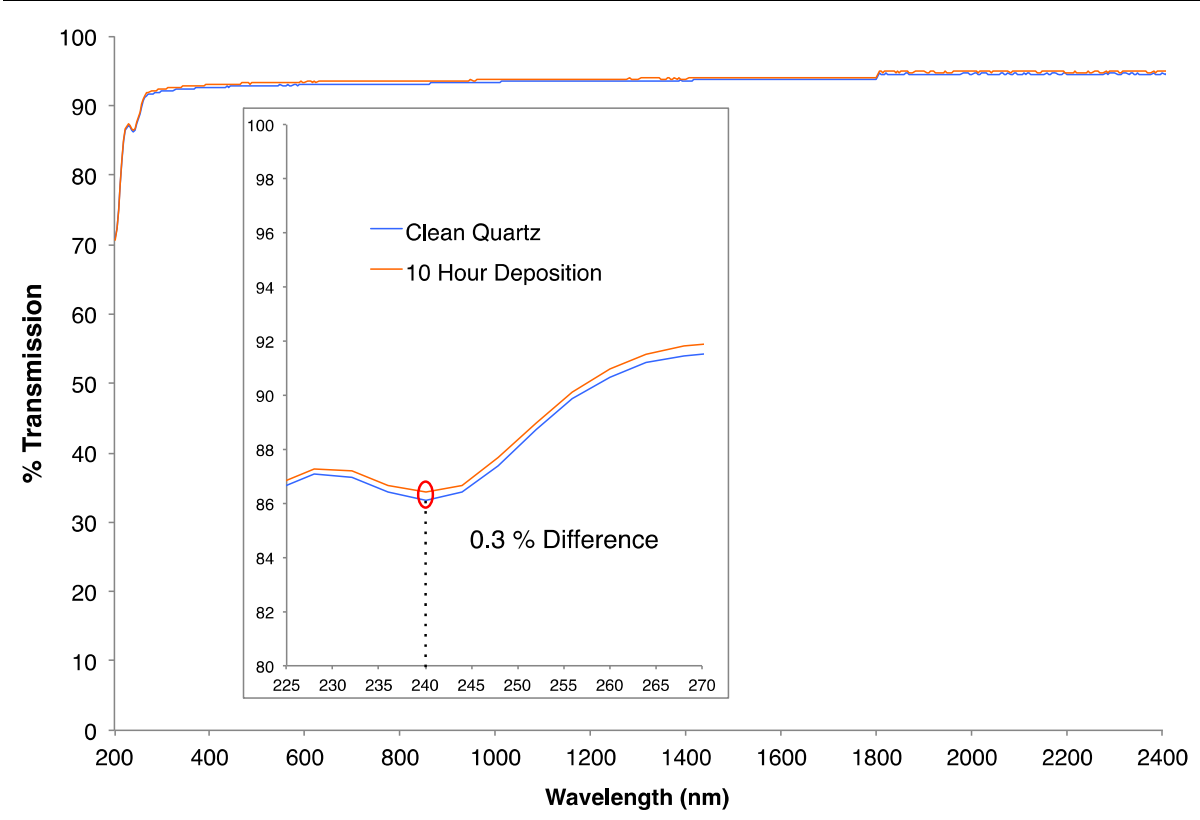

Fig. 73 UV-Near IR spectra of collected residue (200 to $2500 \mathrm{~nm}$ ). This is expanded (insert) in the UV to show the transmission at $240 \mathrm{~nm}$. This is a $0.3 \%$ difference for the $3 \mathrm{ng} / \mathrm{cm}^{2}$ film which falls within the precision of the instrument for this type of measurement rendering the two spectra statistically similar a quartz optic

to $0{ }^{\circ} \mathrm{C}$ for 10.0 hours, $3 \mathrm{~cm}$ from the source. A background sample of the empty chamber deposited onto a sample quartz window was collected for comparison.

The spectral transmission acquired from $200 \mathrm{~nm}$ to $2500 \mathrm{~nm}$ using a Perkin Lambda 1050 UV-Vis-NIR spectrometer is shown in Fig. 73. Post transmission measurement the collected silicone residue on the quartz optic was dissolved in solvent (dichloromethane) and analyzed using Diffuse Reflectance Infrared Fourier Transform (DRIFT) spectroscopy for quantitative determination of the amount of molecular contamination deposited onto the optic. The amount of material deposited was determined to be a $3 \mathrm{ng} / \mathrm{cm}^{2} \mathrm{film}$. Transmittance data were converted to absorbance units and the difference between the two spectra showed no local extrema relative to background, indicating the lack of absorbance events in the wavelength range of interest. This type of analysis often provides a higher precision than the instrument itself, and further supports the results of the transmittance data as within the instrument precision rendering the two spectra as statistically similar.

While analysis and control of materials used in the construction of SHERLOC consumed a significant portion of the contamination control effort, there is recognition that additional mitigation was required to keep deposition of organic material on internal and external optics to below $150 \mathrm{ng} / \mathrm{cm}^{2}$. During the cruise phase of the mission, risk of contamination to optics is greatest. Analysis determined that to minimize condensation of organic material onto optical surfaces, the optical bench would require the use of decontamination heaters during the cruise. The heating scheme is designed to heat the optics to $40{ }^{\circ} \mathrm{C}$ at the beginning of cruise and gradually cool as the spacecraft approaches Mars, while still keeping the optics bench and calibration target approximately $5{ }^{\circ} \mathrm{C}$ warmer than other hardware near or within the line of sight of the instrument. 
An additional mitigation to organic contamination onto the optics incorporates the use of Tenax, poly(2, 6-diphenyl-p-phenylene oxide), as a getter inside the optics bench. Tenax has been analyzed by JPL as a contamination trap for the Adaptive Caching Assembly (ACA) for Mars core sample acquisition. Testing demonstrated that the Tenax material functioned as a molecular absorber in air (during ATLO), vacuum (during cruise) and at 7 Torr $\mathrm{CO}_{2}$ (simulated Martian atmosphere). It also absorbs at temperatures as high as $40^{\circ} \mathrm{C}$. Desorption of Tenax getter is $\sim 200{ }^{\circ} \mathrm{C}$, far above SHERLOC operational temperatures. A particular advantage of Tenax over other molecular absorbers is that it is hydrophobic and does not absorb water. The material also has a high adsorption capacity on the order of $3.7-$ $17.7 \mathrm{mg} / \mathrm{cm}^{2}$, depending upon contaminant. The high adsorptive capacity, coupled with the hydrophobicity of the Tenax, was of particular advantage during ATLO operations.

Vibration testing of the material to simulate worst-case vibration conditions during launch, landing, and Mars surface operations, was performed to evaluate the potential for shedding of particulates inside the optics bench. Analysis of silicon wafer witness plates mounted directly under the Tenax material during testing was found to be free of Tenax particulates on the success of the analyses performed, Tenax was mounted inside the optics bench prior to final closeout as an additional method to mitigate accumulation of organic molecular contamination onto SHERLOC optics.

A rigorous cleaning regimen was implemented for all hardware built for the SHERLOC instrument. Optics and the optical bench were handled inside a dedicated class 10,000 cleanroom with limited access and built on a class 100 laminar flow bench within the cleanroom. Prior to incorporation of parts and optics into the build, thorough inspections were implemented. These inspections included sample swabbing of hardware with hexane to determine surface non-volatile residue (NVR), and Teflon tape lifts to determine particle count as well as visual inspection under magnification. In addition, the cleanroom was constantly monitored with an airborne particle counter, humidity monitor, and temperature monitor. The electronics build was performed inside an adjacent class 10,000 cleanroom and monitored as described above. Both particulate fallout witness plates and separate organic fallout witness plates were used inside both areas to monitor room conditions. Additional particle plates were used to monitor the build of critical optics. Hardware was further protected with the use of a nitrogen-purged box for all piece parts and sub-assemblies during the assembly. Specialized shipping containers and storage containers were also used to minimize contamination to hardware. Hardware monitoring continued in ATLO as Perseverance underwent assembly and test. These samples will be analyzed after launch to document potential contamination and determine their additive effects (if any).

\subsubsection{Hardware Outgassing}

Ultimately, a key factor to maintaining low levels of molecular contamination deposition onto optics was the overall outgassing rate of items within and adjacent to the optics bench. Control of materials, directed venting, use of Tenax getter, incorporation of decontamination heaters, and control of conditions during I\&T are all good mitigation strategies. The outgassing rate of items within the optics bench was required to have an outgassing rate of $1 \mathrm{ng} / \mathrm{cm}^{2} /$ hour (measured with hardware at $+50{ }^{\circ} \mathrm{C}$ and Thermoelectric Quartz Crystal Microbalance, TQCM, at $-50{ }^{\circ} \mathrm{C}$ and chamber at $<10^{-5}$ Torr) overall. Items outside of the optics bench, including electronics boxes, were required to be below $10 \mathrm{ng} / \mathrm{cm}^{2} /$ hour overall. To this end, a rigorous thermal vacuum bakeout campaign with TQCM measurements were made of all hardware on the SHERLOC STA. Piece parts were vacuum baked at high temperatures at low levels of assembly post precision clean, painted/coated items were vacuum baked post paint and coating, harnesses and sub-assemblies were vacuum baked. Many 
piece-part vacuum bakeouts were performed in chambers where the chamber background was monitored with TQCM. However, all final bakeouts and TQCM measurements were performed using a SHERLOC-dedicated vacuum bakeout chamber with a chamber background kept between 1-3 Hz/hour (chamber at $+50{ }^{\circ} \mathrm{C}$ and TQCM at $-50{ }^{\circ} \mathrm{C}$ and $<10^{-5}$ Torr). Having a low chamber background enabled the SHERLOC team to measure accurately the very low outgassing rates of items within the optic bench. Often, the bakeout regime resulted in better than expected outgassing rates for all hardware including electronics.

In summation, the contamination control program for the SHERLOC instrument employed stringent practices for design, build, monitoring of cleanroom environments, thermal vacuum bakeouts, cleaning of hardware, verification of hardware cleanliness, hardware handling, careful selection of materials for build, incorporation of Tenax getter, and incorporation of decontamination heaters in the optics bench. The results to date show that the throughput of the optics is better than expected. It is the anticipation of the contamination control engineers that the optical throughput will continue to be optimal upon arrival on Mars.

\subsection{Planetary Protection}

In order to avoid the forward contamination of Mars from biology from the Earth, hardware landed on Mars must be biologically cleaned. Furthermore, since the Perseverance rover is the first step in sample return, care must be exercised such that samples collected on Mars do not have terrestrial contamination. Planetary Protection referrers to the efforts used to ensure the cleanliness of the Perseverance rover and document all hardware that goes to Mars. The instruments on the robotic arm turret do not come in contact with the Martian surface and SHERLOC and PIXL were designed and tested to meet cleanliness defined in NPR8020.12D, Sect. 5.3.2.1a, and has a maximum total microorganisms limited to $<5100$ spores total for all surfaces that are exposed to the Martian environment over the life cycle of the instrument.

SHERLOC was designed to be a non-contact device to avoid issues with both contamination of returned samples as well as for Planetary Protection. SHERLOC hardware is required to be in conformance with Planetary Protection requirements for the Mars 2020 Project. In addition to total spore count for surfaces exposed to the Martian surface, surfaces behind the HEPA filter have a required maximum bioburden density of $1000 \mathrm{spores} / \mathrm{m}^{2}$ while the exposed surfaces, the maximum bioburden density is limited to 300 spores $/ \mathrm{m}^{2}$.

SHERLOC assembly was performed in clean environments, which were primarily driven by the contamination control requirements. During the assembly process, both the internal and external surfaces were subjected to biological assays prior to its last access using the NASA Standard Assay protocol as described in NASA Handbook 6022. Additionally, credit was also taken for manufacturing processes that resulted in a microbial reduction on the parts that were subject to a high temperature exposure. Following sampling, the hardware was maintained in an ISO 8 or better cleanroom environment to prevent recontamination and double-bagged for transportation. All data collected throughout the life cycle of the instrument through to the final access point was consolidated and the final spore bioburden density was assessed. The results show that SHERLOC surfaces behind the HEPA filter had an average of 14.93 spores $/ \mathrm{m}^{2}$ and 20.4 spores $/ \mathrm{m}^{2}$ on exposed surfaces. This met the requirements of $1000 \mathrm{spores} / \mathrm{m}^{2}$ and 300 spores $/ \mathrm{m}^{2}$, respectively. The total bioburden on exposed surfaces was 38 spores which fell far below the 5100 spore allocation. The SHERLOC instrument met and exceeded expectations set by Planetary Protection for the Mars 2020 Mission. 


\section{Summary}

The NASA Perseverance rover's SHERLOC instrument is designed to help achieve a variety of Mars 2020 mission goals related to exploring an astrobiologically relevant ancient environment within the Jezero crater field site. SHERLOC is a non-contact, robotic arm-mounted instrument that acquires macro and microphotographic images and enables mineralogy and organic molecule detection using laser spectroscopy. The $248.6 \mathrm{~nm}$ laser wavelength has a $100 \mu \mathrm{m}$ laser spot at the target and can be systematically scanned across a surface. Raman scattered photons from organics and minerals are spectrally resolved with a spectral range of $\sim 800-4000 \mathrm{~cm}^{-1}$ ( 250 to $273 \mathrm{~nm}$ ). Fluorescence emitted photons from organics are identifiable in the 270 to $\sim 370 \mathrm{~nm}$ range and are similarly spatially resolved. Individual spectra can be co-located with surface features visible on a grayscale image obtained by the ACI. Additionally, the spectral maps can be placed within color context images obtained by WATSON, overlayed with PIXL elemental maps as well as data from SuperCam and Mastcam-Z multispectral images to fully understand the make-up of an in situ target. Through this process, not only will scientific understanding of the current conditions and the history of Mars be made, but the highest priority samples can be identified for coring and sample caching for eventual return to Earth. This contribution has provided details on the instrument including goals, design, fabrication, testing and expected surface operations for the SHERLOC investigation.

Acknowledgements The work described in this paper was partially carried out at the Jet Propulsion Laboratory, California Institute of Technology, under a contract with the National Aeronautics and Space Administration. We thank the NASA Postdoctoral Program fellowship awarded to Joseph Razzell Hollis, administered by the Universities Space Research Association on behalf of NASA. We thank K. D. Supulver for help with the WATSON motor count to working distance equations. We would thank the two reviewers for reading and improving the paper. We gratefully acknowledge the supporting contributions of Mars 2020 Project Personnel and Review Board members Dave Braun, Tom Glavich, Steve Macenka, Bill Mateer, Glenn Reeves, Jeff Simmonds, Mark Underwood, Mike Wilson, Kevin Clark, Elizabeth Cordoba, Soren Norvang Madsen, Nicole Spanovich, Art Thompson and Steven Scott. We also like to thank all the support of everyone who participated in a review board for any subsystem during the 6 years of instrument design and development. Finally, we wish to give our sincere thanks to everyone who worked the Deep UV spectroscopy technology during the 22 years that it was under development at JPL. It really was a herculean effort done by a group of truly excellent scientists and engineers.

Publisher's Note Springer Nature remains neutral with regard to jurisdictional claims in published maps and institutional affiliations.

Open Access This article is licensed under a Creative Commons Attribution 4.0 International License, which permits use, sharing, adaptation, distribution and reproduction in any medium or format, as long as you give appropriate credit to the original author(s) and the source, provide a link to the Creative Commons licence, and indicate if changes were made. The images or other third party material in this article are included in the article's Creative Commons licence, unless indicated otherwise in a credit line to the material. If material is not included in the article's Creative Commons licence and your intended use is not permitted by statutory regulation or exceeds the permitted use, you will need to obtain permission directly from the copyright holder. To view a copy of this licence, visit http://creativecommons.org/licenses/by/4.0/.

\section{References}

W.J. Abbey, R. Bhartia, L.W. Beegle, L. DeFlores, V. Paez, K. Sijapati, S. Sijapati, K. Williford, M. Tuite, W. Hug, R. Reid, Icarus 290, 201 (2017). https://doi.org/10.1016/j.icarus.2017.01.039

W. Abbey, R. Anderson, L. Beegle, J. Hurowitz, K. Williford, G. Peters, J.M. Morookian, C. Collins, J. Feldman, R. Kinnett, L. Jandura, D. Limonadi, C. Logan, S. McCloskey, J. Melko, A. Okon, M. Robinson, C. Roumeliotis, C. Seybold, J. Singer, N. Warner, Icarus 319, 1 (2019). https://doi.org/10.1016/j.icarus. 2018.09.004 
U. Acuña, F. Amat-Guerri, P. Morcillo, M. Liras, B. Rodríguez, Org. Lett. 11(14), 3020-3023 (2009). https:// doi.org/10.1063/1.5079883

A.C. Allwood, M.R. Walter, B.S. Kamber, C.P. Marshall, I.W. Burch, Nature 441(7094), 714-718 (2006). https://doi.org/10.1038/nature04764

A.C. Allwood, L.A. Wade, M.C. Foote et al., PIXL: Planetary Instrument for X-Ray Lithochemistry. Space Sci. Rev. 216, 134 (2020). https://doi.org/10.1007/s11214-020-00767-7

R.C. Anderson, L.W. Beegle, J. Hurowitz, C. Hanson, W. Abbey, C. Seybold, D. Liminodi, S. Kuhn, L. Jandura, K. Brown, G. Peters, C. Roumeliotis, M. Robinson, K. Edgett, M. Minitti, J. Grotzinger, Icarus 256, 66 (2015). https://doi.org/10.1016/j.icarus.2015.03.033

S.A. Asher, Anal. Chem. 65, 59A (1993). https://doi.org/10.1021/ac00050a001

S.A. Asher, C. Johnson, Science 225(4659), 311-313 (1984). https://doi.org/10.1126/science.6740313

S.A. Asher, J.L. Murtaugh, Appl. Spectrosc. 42, 83 (1988). https://doi.org/10.1366/0003702884428653

B. Balaram, T. Canham, C. Duncan, H.F. Grip, W. Johnson, J. Maki, A. Quon, R. Stern, D. Zhu, Mars helicopter technology demonstrator. AIAA Atmospheric Flight Mechanics Conference, Reston, Virginia (2018). https://doi.org/10.2514/6.2018-0023. Paper AIAA 2018-0023

S.G. Banham, S. Gupta, D.M. Rubin, J.A. Watkins, D.Y. Sumner, K.S. Edgett, J.P. Grotzinger, K.W. Lewis, L.A. Edgar, K.M. Stack-Morgan, R. Barnes, J.F. Bell, M.D. Day, R.C. Ewing, M.G.A. Lapotre, N.T. Stein, F. Rivera-Hernandez, A.R. Vasavada, Sedimentology 65, 993 (2018). https://doi.org/10.1111/sed. 12469

B.E. Bayer, Color imaging array. United States Patent 3,971,065 (1976)

L.W. Beegle, R. Bhartia, M. White, L. DeFlores, W. Abbey, Y.-H. Wu, B. Cameron, J. Moore, M. Fries, A. Burton, K.S. Edgett, M.A. Ravine, W. Hug, R. Reid, T. Nelson, S. Clegg, R. Wiens, S. Asher, P. Sobron, SHERLOC: Scanning habitable environments with Raman \& luminescence for organics \& chemicals, in 2015 IEEE Aerospace Conference (IEEE, Big Sky, 2015). https://doi.org/10.1109/AERO.2015.7119105

J.F. Bell III, J.N. Maki, G.L. Mehall, M.A. Ravine, M.A. Caplinger, Z.J. Bailey, S. Brylow, J.A. Schaffner, K.M. Kinch, M.B. Madsen, A. Winhold, A.G. Hayes, P. Corlies, C. Tate, M. Barrington, E. Cisneros, E. Jensen, K. Paris, K. Crawford, C. Rojas, L. Mehall, J. Joseph, J.B. Proton, N. Cluff, R. Deen, B. Betts, E. Cloutis, A.J. Coates, A. Colaprete, K.S. Edgett, B.L. Ehlmann, S. Fagents, J.P. Grotzinger, C. Hardgrove, K.E. Herkenhoff, B. Horgan, R. Jaumann, J.R. Johnson, M. Lemmon, G. Paar, M. Caballo-Perucha, S. Gupta, C. Traxler, F. Preusker, M.S. Rice, M.S. Robinson, N. Schmitz, R. Sullivan, M.J. Wolff, The Mars 2020 perseverance rover mast camera zoom (Mastcam-Z) multispectral, stereoscopic imaging investigation. Space Sci. Rev. (2020, this journal). https://doi.org/10.1007/s11214-020-00755-X

S.A. Benner, K.G. Devine, L.N. Matveeva, D.H. Powell, The missing organic molecules on Mars. Proc. Natl. Acad. Sci. USA 97(6), 2425-2430 (2000). https://doi.org/10.1073/pnas.040539497

R. Bhartia, Non invasive detection of microbes in natural environments using deep UV native fluorescence spectroscopy and hyperspectral imaging. Ph.D. Thesis, University of Southern California (2012). uscthesesreloadpub_Volume71/etd-BhartiaRoh-545.pdf

R. Bhartia, W. Hug, E. Salas, R. Reid, K. Sijapati, A. Tsapin, W. Abbey, K. Nealson, A. Lane, P. Conrad, Appl. Spectrosc. 62(10), 1070-1077 (2008). https://doi.org/10.1366/000370208786049123

R. Bhartia, E. Salas, W. Hug, R. Reid, A. Lane, K. Edwards, K. Nealson, Label-free bacterial imaging with deep-UV-laser-induced native fluorescence. Appl. Environ. Microbiol. 76(21), 7231-7237 (2010). https://doi.org/10.1128/AEM.00943-10

R. Bhartia, W. Hug, R. Reid, Improved sensing using simultaneous deep UV Raman and fluorescence detection. Proc. SPIE 8358, 83581A (2012a). https://doi.org/10.1117/12.920170

R. Bhartia, W. Hug, R. Reid, E. Salas SPIE Defense (2012b). https://doi.org/10.1117/12.921420

R. Bhartia, W. Hug, R. Reid, L. Beegle, Explosives detection and analysis by fusing deep UV native fluorescence and resonance Raman spectroscopy, in Laser-Based Optical Detection Methods of Explosives, ed. by P.M. Pellegrino, E.L. Holthoff, M.E. Ferrell (Taylor \& Francis Group, Boca Raton, 2014)

R. Borkman, D. Kearns, Electronic relaxation processes in acetone. J. Chem. Phys. 44, 945 (1966). https:// doi.org/10.1063/1.1726846

I.S. Bowen, Wave lengths of forbidden nebular lines. II. Astrophys. J. 132(1), 1-17 (1960). https://doi.org/ $10.1086 / 146893$

S.L. Cady, J.R. Skok, V.G. Gulick, J.A. Berger, N.W. Hinman, Siliceous hot spring deposits: Why they remain key astrobiological targets, in From Habitability to Life on Mars (2018), pp. 179-210. https://doi.org/ 10.1016/B978-0-12-809935-3.00007-4. Chap. 7

L. Cameron, The Geocaching Handbook: The Guide for Family-Friendly High-Tech Treasure Hunting, 3rd edn. (Falcon Guides, Guilford, 2017). 152 p.

G. Caravaca, N. Mangold, S. Le Mouélic, L. Le Deit, O. Gasnault, F. Rivera-Hernandez, C.M. Fedo, K.S. Edgett, R.C. Wiens, Characterization of small sedimentary structures in rocks of the Glen Torridon region (Gale crater, Mars) using photogrammetry, in Lunar Planet. Sci. Conf., vol. 51, abstract 1455 (2020) 
M.H. Carr, J.W. Head III, Oceans on Mars: An assessment of the observational evidence and possible fate. J. Geophys. Res., Planets 108(E5), 5042 (2003). https://doi.org/10.1029/2002JE001963

B. Carrier, W.J. Abbey, L.W. Beegle, R. Bhartia, Y. Liu, Attenuation of ultraviolet radiation in rocks and minerals: Implications for Mars science. J. Geophys. Res., Planets 124, 2599-2612 (2019). https://doi. org/10.1029/2018JE005758

S. Chang, D. Des Marais, R. Mack, S.L. Miller, G.E. Strathearn, Pre-biotic organic synthesis and the origin of life, in Earth's Earliest Biosphere: Its Origin and Evolution, ed. by J.W. Schopf (Princeton University Press, Princeton, 1983), pp. 53-92

H. Chennaoui Aoudjehane, G. Avice, J.A. Barrat, O. Boudouma, G. Chen, M.J.M. Duke, I.A. Franchi, J. Gattacecca, M.M. Grady, R.C. Greenwood, C.D.K. Herd, R. Hewins, A. Jambon, B. Marty, P. Rochette, C.L. Smith, V. Sautter, A. Verchovsky, P. Weber, B. Zanda, Tissint Martian meteorite: A fresh look at the interior, surface, and atmosphere of Mars. Science 338(6108), 785-788 (2012). https://doi.org/10. 1126/science. 1224514

S. Christesen, J. Jones, J. Lochner, Optics InfoBase: Applied spectroscopy - Ultraviolet Raman spectra and cross-sections of the G-series nerve agents. Appl. Spectrosc. 62(10), 1078-1083 (2008). https://doi.org/ 10.1366/000370208786049024

L. Csillag, M. Janossy, V. Rosa, T. Salamon, New infrared CW laser oscillation in Cu II. Phys. Lett. A 50, 13-14 (1974). https://doi.org/10.1016/0375-9601(74)90331-4

D. Czaja, A.B. Kudryavtsev, G.D. Cody, J.W. Schopf, Org. Geochem. 40, 353 (2009). https://doi.org/10.1016/ j.orggeochem.2008.12.002

F.J. de Hoog, J.R. McNeil, G.J. Collins, K.B. Persson, Discharge studies of the Ne-Cu laser. J. Appl. Phys. 48, 3701-3704 (1977). https://doi.org/10.1063/1.324284

C. de la Torre, J.d.V. Albendea, Digital correlated double sampling CCD readout characterization, in Proc. SPIE 10709, High Energy, Optical, and Infrared Detectors for Astronomy VIII, 1070920 (2018). https:// doi.org/10.1117/12.2312498

J.D. Deane, Ichnographs from the Sandstone of Connecticut River (Little, Brown, Boston, 1861)

D.R. DiBiase, J. Laramee, Mars Hand Lens Imager: Lens mechanical design, in Proc. 2009 IEEE Aerospace Conf., Paper 1215 (2009). https://doi.org/10.1109/AERO.2009.4839434

K.S. Edgett, H.E. Newsom, Dust deposited from eolian suspension on natural and spaceflight hardware surfaces in Gale crater as observed using Curiosity's Mars Hand Lens Imager (MAHLI), in Dust in the Atmosphere of Mars and Its Impact on Human Exploration, ed. by J.S. Levine, D. Winterhalter, R.L. Kerschmann (Cambridge Scholars Publishing, Newcastle upon Tyne, 2018), pp. 81-104

K.S. Edgett, R.A. Yingst, M.A. Ravine, M.A. Caplinger, J.N. Maki, F.T. Ghaemi, J.A. Schaffner, J.F. Bell III, L.J. Edwards, K.E. Herkenhoff, E. Heydari, L.C. Kah, M.T. Lemmon, M.E. Minitti, T.S. Olson, T.J. Parker, S.K. Rowland, J. Schieber, R.J. Sullivan, D.Y. Sumner, P.C. Thomas, E.H. Jensen, J.J. Simmonds, A.J. Sengstacken, R.G. Willson, W. Goetz, Curiosity's Mars Hand Lens Imager (MAHLI) investigation. Space Sci. Rev. 170, 259-317 (2012). https://doi.org/10.1007/s11214-012-9910-4

K.S. Edgett, M.A. Caplinger, J.N. Maki, M.A. Ravine, F.T. Ghaemi, S. McNair, K.E. Herkenhoff, B.M. Duston, R.G. Willson, R.A. Yingst, M.R. Kennedy, M.E. Minitti, A.J. Sengstacken, K.D. Supulver, L.J. Lipkaman, G.M. Krezoski, M.J. McBride, T.L. Jones, B.E. Nixon, J.K. Van Beek, D.J. Krysak, R.L. Kirk, Curiosity's robotic arm-mounted Mars Hand Lens Imager (MAHLI): Characterization and calibration status, version 2, 05 October 2015. MSL MAHLI Tech. Rept. 0001 (2015). https://doi.org/ 10.13140/RG.2.1.3798.5447

K.S. Edgett, M.A. Caplinger, M.A. Ravine, in Mars 2020 Perseverance SHERLOC WATSON Camera Predelivery Characterization and Calibration Report (Malin Space Science Systems, San Diego, California (2019). https://doi.org/10.13140/RG.2.2.18447.00165

B.L. Ehlmann et al., Orbital identification of carbonate-bearing rocks on Mars. Science 322(5909), 1828-1832 (2008) https://doi.org/10.1126/science.1164759

B.L. Ehlmann et al., Subsurface water and clay mineral formation during the early history of Mars. Nature 479(7371), 53-60 (2011). https://doi.org/10.1038/nature10582

B.L. Ehlmann, K.S. Edgett, B. Sutter, C.N. Achilles, M.L. Litvak, M.G.A. Lapotre, R. Sullivan, A.A. Fraeman, R.E. Arvidson, D.F. Blake, N.T. Bridges, P.G. Conrad, A. Cousin, R.T. Downs, T.S.J. Gabriel, R. Gellert, V.E. Hamilton, C. Hardgrove, J.R. Johnson, S. Kuhn, P.R. Mahaffy, S. Maurice, M. McHenry, P.-Y. Meslin, D.W. Ming, M.E. Minitti, J.M. Morookian, R.V. Morris, C.D. O’Connell-Cooper, P.C. Pinet, S.K. Rowland, S. Schröder, K.L. Siebach, N.T. Stein, L.M. Thompson, D.T. Vaniman, A.R. Vasavada, D.F. Wellington, R.C. Wiens, A.S. Yen, J. Geophys. Res., Planets 122, 2510 (2017). https:// doi.org/10.1002/2017JE005267

J.L. Eigenbrode, R.E. Summons, A. Steele, C. Freissinet, M. Millan, R. Navarro-González, B. Sutter et al., Organic matter preserved in 3-billion-year-old mudstones at Gale crater, Mars. Science 360(6393), 1096-1101 (2018). https://doi.org/10.1126/science.aas9185 
K.A. Farley, K.H. Williford, K.M. Stack et al., Mars 2020 mission overview. Space Sci. Rev. 216, 142 (2020). https://doi.org/10.1007/s11214-020-00762-y

C.I. Fassett, J.W. Head III., Fluvial sedimentary deposits on Mars: Ancient deltas in a crater lake in the Nili Fossae region. Geophys. Res. Lett. 32, L14201 (2005). https://doi.org/10.1029/2005GL023456

M.R. Fisk, J. Pommerenck, K. Watkins-Brandt, K.S. Edgett, M.E. Minitti, C.J. Hardgrove, R. Popa, W. Goetz, E. Cloutis, B. Nixon, M.R. Kennedy, A.H. Treiman, in AGU Fall Meeting Abstracts, vol. 51, P51E (2015)

G.J. Flynn, The delivery of organic matter from asteroids and comets to the early surface of Mars, in Worlds in Interaction: Small Bodies and Planets of the Solar System (Springer, Dordrecht, 1996), pp. 469-474. https://doi.org/10.1007/978-94-009-0209-1_58

G.J. Flynn, D.S. McKay, An assessment of the meteoritic contribution to the Martian soil. J. Geophys. Res., Solid Earth 95(B9), 14497-14509 (1990). https://doi.org/10.1029/JB095iB09p14497

M.A. Fonstad, A. Zettler-Mann, The camera and the geomorphologist. Geomorphology 366, 107181 (2020). https://doi.org/10.1016/j.geomorph.2020.107181

T. Fornaro, A. Steele, J. Brucato, Catalytic/protective properties of Martian minerals and implications for possible origin of life on Mars. Life 8, 56 (2018). https://doi.org/10.3390/life8040056

C. Freissinet, D.P. Glavin, P.R. Mahaffy, K.E. Miller, J.L. Eigenbrode, R.E. Summons, A.E. Brunner et al., Organic molecules in the sheepbed mudstone, Gale crater, Mars. J. Geophys. Res., Planets 120(3), 495-514 (2015). https://doi.org/10.1002/2014JE004737

M. Fries, A. Steele, in Confocal Raman Microscopy, ed. by J. Toporski, T. Dieing, O. Hollricher (Springer, Cham, 2018), pp. 209-236

M. Fries, R. Bhartia, A. Steele, Carbonaceous chondrite groups discerned using Raman spectral parameters, in Lunar and Planetary Science Conference, Houston, Tx, vol. 42 (2011), p. 1860. https://doi.org/10. 1016/j.gca.2016.09.024

T. Frosch, N. Tarcea, M. Schmitt, H. Thiele, F. Langenhorst, J. Popp, Anal. Chem. 79, 1101 (2007). https:// doi.org/10.1021/ac0618977

R.H. Garstang, Transition probabilities for forbidden lines of Fe III and Fe V. Mon. Not. R. Astron. Soc. 117, 393 (1957)

J.B. Garvin, K.S. Edgett, R. Dotson, D.M. Fey, K.E. Herkenhoff, B.J. Hallet, M.R. Kennedy, Quantitative relief models of rock surfaces on Mars at sub-millimeter scales from Mars Curiosity rover Mars Hand Lens Imager (MAHLI) observations: Geologic implications. Microsc. Microanal. 23(S1), 2146-2147 (2017). https://doi.org/10.1017/S1431927617011394

R. Gellert, B.C. Clark III, MSL and MER Science Teams, In situ compositional measurements of rocks and soils with the Alpha Particle X-Ray Spectrometer on NASA's Mars rovers. Elements 11, 39-44 (2015). https://doi.org/10.2113/gselements.11.1.39

F.T. Ghaemi, Design and fabrication of lenses for the color science cameras aboard the Mars Science Laboratory rover. Opt. Eng. 48(10), 103002 (2009). https://doi.org/10.1117/1.3251343

D.P. Glavin, C. Freissinet, K.E. Miller, J.L. Eigenbrode, A.E. Brunner, A. Buch, B. Sutter et al., Evidence for perchlorates and the origin of chlorinated hydrocarbons detected by SAM at the Rocknest aeolian deposit in Gale Crater. J. Geophys. Res., Planets 118(10), 1955-1973 (2013). https://doi.org/10.1002/ jgre.20144

T.D. Glotch et al., Distribution and formation of chlorides and phyllosilicates in Terra Sirenum, Mars. Geophys. Res. Lett. 37, 1-5 (2010). https://doi.org/10.1126/science.1164759

W. Goetz, W.T. Pike, S.F. Hviid, M.B. Madsen, R.V. Morris, M.H. Hecht, U. Staufer, K. Leer, H. Sykulska, E. Hemmig, J. Marshall, J.M. Morookian, D. Parrat, S. Vijendran, B.J. Bos, M.R. El Maarry, H.U. Keller, R. Kramm, W.J. Markiewicz, L. Drube, D. Blaney, R.E. Arvidson, J.F. Bell III, R. Reynolds, P.H. Smith, P. Woida, R. Woida, R. Tanner, Microscopy analysis of soils at the Phoenix landing site, Mars: Classification of soil particles and description of their optical and magnetic properties. J. Geophys. Res., Planets 115, E00E22 (2010). https://doi.org/10.1029/2009JE003437

Golder et al., Characteristics of dark-toned crater floor materials, Jezero crater: Implications for mode of emplacement, in Lunar Planet. Sci. Conf., vol. 51 (Lunar and Planetary Institute, Houston, Texas, 2020), p. 1302.

T.A. Goudge, J.F. Mustard, J.W. Head, C.I. Fassett, Constraints on the history of open-basin lakes on Mars from the composition and timing of volcanic resurfacing. J. Geophys. Res., Planets 117, E00J21 (2012). https://doi.org/10.1029/2012JE004115

T.A. Goudge, J.F. Mustard, J.W. Head, C.I. Fassett, S.M. Wiseman, Assessing the mineralogy of the watershed and fan deposits of the Jezero crater paleolake system, Mars. J. Geophys. Res., Planets 120, 775-808 (2015). https://doi.org/10.1002/2014JE004782

T.A. Goudge, R.E. Milliken, J.W. Head, J.F. Mustard, C.I. Fassett, Sedimentological evidence for a deltaic origin of the western fan deposit in Jezero crater, Mars and implications for future exploration. Earth Planet. Sci. Lett. 458, 357-365 (2017). https://doi.org/10.1016/j.epsl.2016.10.056 
M.M. Grady, The history of research on meteorites from Mars, in History of Meteoritics and Key Meteorite Collections: Fireballs, Falls and Finds, ed. by G.J. McCall, A.J. Bowden, R.J. Howarth (Geological Society Publishing House, Bath, 2006), pp. 405-416. https://doi.org/10.1111/j.1945-5100.2007.tb00244.X

M.M. Grady, I.P. Wright, C.T. Pillinger, A carbon and nitrogen isotope study of Zagami. J. Geophys. Res., Planets 102(E4), 9165-9173 (1997). https://doi.org/10.1029/97JE00414

M.M. Grady, A.B. Verchovsky, I.P. Wright, Magmatic carbon in Martian meteorites: Attempts to constrain the carbon cycle on Mars. Int. J. Astrobiol. 3, 117-124 (2004). https://doi.org/10.1017/ S1473550404002071

J.A. Grant, M.P. Golombek, S.A. Wilson, K.A. Farley, K.H. Williford, A. Chen, The science process for selecting the landing site for the 2020 Mars rover. Planet. Space Sci. 164, 106-126 (2018). https:// doi.org/10.1016/j.pss.2018.07.001

J.P. Grotzinger, J. Crisp, A.R. Vasavada, R.C. Anderson, C.J. Baker, R. Barry, D.F. Blake, P. Conrad, K.S. Edgett, B. Ferdowski, R. Gellert, J.B. Gilbert, M. Golombek, J. Gómez-Elvira, D.M. Hassler, L. Jandura, M. Litvak, P. Mahaffy, J. Maki, M. Meyer, M.C. Malin, I. Mitrofanov, J.J. Simmonds, D. Vaniman, R.V. Welch, R.C. Wiens, Mars Science Laboratory mission and science investigation. Space Sci. Rev. 170, 5-56 (2012). https://doi.org/10.1007/s11214-012-9892-2

J.P. Grotzinger, D.Y. Sumner, L.C. Kah, K. Stack, S. Gupta, L. Edgar, D. Rubin, K. Lewis, J. Schieber, N. Mangold, R. Milliken, P.G. Conrad, D. DesMarais, J. Farmer, K. Siebach, F. Calef, J. Hurowitz, S.M. McLennan, D. Ming, D. Vaniman, J. Crisp, A. Vasavada, K.S. Edgett, M. Malin, D. Blake, R. Gellert, P. Mahaffy, R.C. Wiens, S. Maurice, J.A. Grant, S. Wilson, R.C. Anderson, L. Beegle, R. Arvidson, B. Hallet, R.S. Sletten, M. Rice, J. Bell, J. Griffes, B. Ehlmann, R.B. Anderson, T.F. Bristow, W.E. Dietrich, G. Dromart, J. Eigenbrode, A. Fraeman, C. Hardgrove, K. Herkenhoff, L. Jandura, G. Kocurek, S. Lee, L.A. Leshin, R. Leveille, D. Limonadi, J. Maki, S. McCloskey, M. Meyer, M. Minitti, H. Newsom, D. Oehler, A. Okon, M. Palucis, T. Parker, S. Rowland, M. Schmidt, S. Squyres, A. Steele, E. Stolper, R. Summons, A. Treiman, R. Williams, A. Yingst (MSL Science Team), A habitable fluvio-lacustrine environment at Yellowknife Bay, Gale crater, Mars. Science 343, 1242777 (2014). https://doi.org/10. $1126 /$ science. 1242777

S.E. Hamran, D.A. Paige, H.E.F. Amundsen et al., Radar Imager for Mars' Subsurface Experiment-RIMFAX. Space Sci. Rev. 216, 128 (2020). https://doi.org/10.1007/s11214-020-00740-4

T.M. Harrison, Hadean Earth (Springer, Berlin, 2020). https://doi.org/10.1007/978-3-030-46687-9

H. Hartman, S. Johansson, Ultraviolet fluorescence lines of Fe II observed in satellite spectra of the symbiotic star RR Telescopii. Astron. Astrophys. 359(2), 627-634 (2000)

M.H. Hecht, S.P. Kounaves, R.C. Quinn, S.J. West, S.M. Young, D.W. Ming, D.C. Catling, B.C. Clark, W.V. Boynton, J. Hoffman, L.P. Deflores, K. Gospodinova, J. Kapit, P.H. Smith, Detection of perchlorate and the soluble chemistry of martian soil at the Phoenix lander site. Science 325(5936), 64-67 (2009). https://doi.org/10.1126/science.1172466

K.E. Herkenhoff, S.W. Squyres, J.F. Bell III, J.N. Maki, H.M. Arneson, P. Bertelson, D.I. Brown, S.A. Collins, A. Dingizian, S.T. Elliot, W. Goetz, E.C. Hagerott, A.G. Hayes, M.J. Johnson, R.L. Kirk, S. McLennan, R.V. Morris, L.M. Scherr, M.A. Schwochert, L.R. Shiraishi, G.H. Smith, L.A. Soderblom, J.N. Sohl-Dickstein, M.V. Wadsworth, Athena microscopic imager investigation. J. Geophys. Res., Planets 108(E12), 8065 (2003). https://doi.org/10.1029/2003JE00207

K.E. Herkenhoff, S.W. Squyres, R. Anderson, B.A. Archinal, R.E. Arvidson, J.M. Barrett, K.J. Becker, J.F. Bell III, C. Budney, N.A. Cabrol, M.G. Chapman, D. Cook, B.L. Ehlmann, J. Farmer, B. Franklin, L.R. Gaddis, D.M. Galuszka, P.A. Garcia, T.M. Hare, E. Howington-Kraus, J.R. Johnson, S. Johnson, K. Kinch, R.L. Kirk, E.M. Lee, C. Leff, M. Lemmon, M.B. Madsen, J.N. Maki, K.F. Mullins, B.L. Redding, L. Richter, M.R. Rosiek, M.H. Sims, L.A. Soderblom, N. Spanovich, R. Springer, R.M. Sucharski, T. Sucharski, R. Sullivan, J.M. Torson, A. Yen, Overview of the microscopic imager investigation during spirit's first 450 sols in Gusev crater. J. Geophys. Res., Planets 111, E02S04 (2006). https://doi.org/10. 1029/2005JE002574

K.E. Herkenhoff, S.W. Squyres, R.E. Arvidson, S.B. Cole, R. Sullivan, A. Yingst, N. Cabrol, E.M. Lee, J. Richie, B. Sucharski, J.F. Bell III, F. Calef, M. Chapman, L. Edgar, B. Franklin, P. Geissler, J. Hurowitz, E. Jensen, J.R. Johnson, R. Kirk, P. Lanagan, C. Leff, J. Maki, K. Mullins, B. Redding, M. Rice, M. Sims, L. Soderblom, N. Spanovich, R. Springer, A. Sunda, A. Vaughan, Overview of spirit microscopic imager results. J. Geophys. Res., Planets 124, 528-584 (2019). https://doi.org/10.1029/2018JE005774

M. Höhl, M. Meinhardt-Wollweber, H. Schmitt, T. Lenarz, U. Morgner, in Biomedical Vibrational Spectroscopy 2016: Advances in Research and Industry, ed. by A. Mahadevan-Jansen, W. Petrich, San Francisco, California, United States (2016), p. 970404

B.H. Horgan, R.B. Anderson, G. Dromart, E.S. Amador, M.S. Rice, The mineral diversity of Jezero crater: Evidence for possible lacustrine carbonates on Mars. Icarus 339, 113526 (2020). https://doi.org/10. 1016/j.icarus. 2019.113526 
W. Hug, R. Reid, R. Bhartia, A. Lane, Performance status of a small robot-mounted or hand-held, solar-blind, standoff chemical, biological, and explosives (CBE) sensor. Proc. SPIE 7304, 73040Z (2009). https:// doi.org/10.1117/12.817881

W.F. Hug, R. Bhartia, R.D. Reid, M.R. Reid, P. Oswal, A.L. Lane, K. Sijapati, K. Sullivan, J.E. Hulla, J. Snawder, S.P. Proctor, Wearable real-time direct reading naphthalene and VOC personal exposure monitor. In: Proceedings of Spie - the International Society For Optical Engineering, vol. 8366 (2012). https://doi.org/10.1117/12.918945

W. Hug, R. Bhartia, K. Sijapati, Q. Nguyen, P. Oswal, R. Reid, Rapid optical detection and classification of microbes in suspicious powders. Proc. SPIE 10629, 106290J (2018). https://doi.org/10.1117/ 12.2299856

J. Janesick, Scientific Charge-Coupled Devices (SPIE Press Books, Bellingham, 2001)

J.R. Janesick, K.P. Klaasen, T. Elliott, Charge-coupled-device charge-collection efficiency and the photontransfer technique. Opt. Eng. 26, 972-980 (1987). https://doi.org/10.1117/12.7974183

E. Jarosewich, R.S. Clarke Jr., J.N. Barrows, Allende meteorite reference sample, in Smithsonian Contributions to the Earth Sciences (1987), pp. 1-49. https://doi.org/10.5479/si.00810274.27.1

S. Johansson, T. Zethson, H. Hartman, J.O. Ekberg, K. Ishibashi, K. Davidson, T. Gull, Astron. Astrophys. 361(3), 977-981 (2000)

C. Johnson, S. Asher, UV resonance Raman excitation profiles of l-cystine. J. Raman Spectrosc. 18, 345-349 (1987). https://doi.org/10.1002/jrs.1250180509.

L.C. Kah, K.M. Stack, J.L. Eigenbrode, R.A. Yingst, K.S. Edgett, Terra Nova 30, 431 (2018)

H.U. Keller, W. Goetz, H. Hartwig, S.F. Hviid, R. Kramm, W.J. Markiewicz, R. Reynolds, C. Shinohara, P. Smith, R. Tanner, P. Woida, R. Woida, B.J. Bos, M.T. Lemmon, Phoenix robotic arm camera. J. Geophys. Res., Planets 113, E00A17 (2008). https://doi.org/10.1029/2007JE003044

H. Kerp, B. Bomfleur, Photography of plant fossils-New techniques, old tricks. Rev. Palaeobot. Palynol. 166, 117-151 (2011). https://doi.org/10.1016/j.revpalbo.2011.05.001

K.M. Kinch, M.B. Madsen, J.F. Bell III, J.N. Maki, Z. Bailey, A.G. Hayes, O.B. Jensen, M. Merusi, M.H. Bernt, A.N. Sørensen, M. Hilverda, E. Cloutis, D. Applin, E. Mateo-Marti, J.A. Manrique, G. LopezReyes, A. Bello-Arufe, B.L. Ehlmann, J. Buz, A. Pommerol, N. Thomas, L. Affolter, K. Herkenhoff, J.R. Johnson, M. Rice, P. Corlies, C. Tate, M. Caplinger, E. Jensen, T. Kubacki, E. Cisneros, K. Paris, A. Winhold, Radiometric calibration targets for the Mastcam-Z camera on the Mars 2020 rover mission. Space Sci. Rev. (2020, this journal). https://doi.org/10.1007/s11214-020-00774-8

G. Kminek, J.L. Bada, The effect of ionizing radiation on the preservation of amino acids on Mars. Earth Planet. Sci. Lett. 245(1-2), 1-5 (2006). https://doi.org/10.1016/j.eps1.2006.03.008

C.H. Kremer, J.F. Mustard, M.S. Bramble, A widespread olivine-rich ash deposit on Mars. Geology 47(7), 677-681 (2019). https://doi.org/10.1130/G45563.1

W.L. Kubasek, B. Hudson, W.L. Peticolas, Proc. Natl. Acad. Sci. USA 82, 2369 (1985). https://doi.org/10. 1073/pnas.82.8.2369

S. Le Mouélic, O. Gasnault, K.E. Herkenhoff, N.T. Bridges, Y. Langevin, N. Mangold, S. Maurice, R.C. Wiens, P. Pinet, H.E. Newsom, R.G. Deen, J.F. Bell III, J.R. Johnson, W. Rapin, B. Barraclough, D.L. Blaney, L. Deflores, J. Maki, M.C. Malin, R. Pérez, M. Saccoccio, The ChemCam remote micro-imager at Gale crater: Review of the first year of operations on Mars. Icarus 249, 93-107 (2015). https://doi. org/10.1016/j.icarus.2014.05.030

E. Le Ru, P. Etchegoin, Principles of Surface Enchanced Raman Spectroscopy (Elsevier, Amsterdam, 2008). https://doi.org/10.1016/B978-0-444-52779-0.X0001-3

D.A. Long, Raman Spectroscopy (McGraw-Hill, New York, 1977). ISBN 0070386757

P.R. Mahaffy, C.R. Webster, M. Cabane, P.G. Conrad, P. Coll, S.K. Atreya, R. Arvey et al., The sample analysis at Mars investigation and instrument suite. Space Sci. Rev. 170(1-4), 401-478 (2012)

J.N. Maki, J.F. Bell III., K.E. Herkenhoff, S.W. Squyres, A. Kiely, M. Klimesh, M. Schwochert, T. Litwin, R. Willson, A. Johnson, M. Maimone, E. Baumgartner, A. Collins, M. Wadsworth, S.T. Elliot, A. Dingisian, D. Brown, E.C. Hagerott, L. Scherr, R. Deen, D. Alexander, J. Lorre, Mars Exploration Rover engineering cameras. J. Geophys. Res., Planets 108(E12), 8071 (2003). https://doi.org/10.1029/2003JE002077

J.N. Maki, D. Gruel, C. McKinney, M.A. Ravine, M. Morales, D. Lee, R. Willson, D. Copley-Woods, M. Valvo, T. Goodsall, J. McGuire, R.G. Sellar, J.A. Schaffner, M.A. Caplinger, J.M. Shamah, A.E. Johnson, H. Ansari, K. Singh, T. Litwin, R. Deen, A. Culver, N. Ruoff, D. Petrizzo, D. Kessler, C. Basset, T. Estlin, F. Alibay, A. Nelessen, S. Algermissen, The Mars 2020 engineering cameras and microphone on the Perseverance rover: A next-generation imaging system for Mars exploration. Space Sci. Rev. (2020, this journal). https://doi.org/10.1007/s11214-020-00765-9

M.J. Malaska, R. Bharita, K.S. Manatt et al., Subsurface In Situ Detection of Microbes and Diverse Organic Matter Hotspots in the Greenland Ice Sheet. Astrobiology 20(12), 1185-1211 (2020). https://doi.org/10. 1089/ast.2020.2241

T.S. Maley, Field Geology Illustrated (Mineral Land Publications, Boise, 2005). 
M.C. Malin, M.A. Ravine, M.A. Caplinger, F.T. Ghaemi, J.A. Schaffner, J.N. Maki, J.F. Bell III, J.F. Cameron, W.E. Dietrich, K.S. Edgett, L.J. Edwards, J.B. Garvin, B. Hallet, K.E. Herkenhoff, E. Heydari, L.C. Kah, M.T. Lemmon, M.E. Minitti, T.S. Olson, T.J. Parker, S.K. Rowland, J. Schieber, R. Sletten, R.J. Sullivan, D.Y. Sumner, R.A. Yingst, B.M. Duston, S. McNair, E.H. Jensen, The Mars Science Laboratory (MSL) mast cameras and descent imager: Investigation and instrument descriptions. Earth Space Sci. 4, 506-539 (2017). https://doi.org/10.1002/2016EA000252

N. Mangold, F. Poulet, J.F. Mustard, J.-P. Bibring, B. Gondet, Y. Langevin, V. Ansan, Ph. Masson, C. Fassett, J.W. Head III., H. Hoffmann, G. Neukum, Mineralogy of the Nili Fossae region with OMEGA/Mars Express data: 2. Aqueous alteration of the crust. J. Geophys. Res., Planets 112, E08S04 (2007). https:// doi.org/10.1029/2006JE002835

S. Maurice, R.C. Wiens, P. Bernardi et al., The SuperCam instrument suite on the Mars 2020 Rover: Science objectives and mast-unit description. Space Sci. Rev. 217, 47 (2021). https://doi.org/10.1007/s11214021-00807-w

J.R. McNeil, G.J. Collins, K.B. Persson, D.L. Franzen, CW laser oscillation in Cu II. Appl. Phys. Lett. 27, 595-598 (1975). https://doi.org/10.1063/1.88300

J.R. McNeil, G.J. Collins, K.B. Persson, D.L. Franzen, Ultraviolet laser action from Cu II in the $2500 \AA$ region. Appl. Phys. Lett. 28, 207-209 (1976). https://doi.org/10.1063/1.88698

J. Michalski, P.B. Niles, Atmospheric origin of Martian interior layered deposits: Links to climate change and the global sulfur cycle. Geology 40(5), 419-422 (2012). https://doi.org/10.1130/G32971.1

J.R. Michalski, J. Cuadro, P.B. Niles, J. Parnell, A.D. Rogers, S.P. Wright, Groundwater activity on Mars and implications for a deep biosphere. Nat. Geosci. 6, 133-138 (2013). https://doi.org/10.1038/ngeo1706

N. Miklusicak, J. Atkins, B. Boring, T. Hicks, E. Shaver, R. Spaulding, K. Golder, W. Abbey, L. Kah, Large fractures in Jezero crater: Implications for understanding dark-toned crater floor materials, in Lunar Planet. Sci. Conf., vol. 51 (Lunar and Planetary Institute, Houston, Texas, 2020), p. 1303

M.E. Minitti, L.C. Kah, R.A. Yingst, K.S. Edgett, R.C. Anderson, L.W. Beegle, J.L. Carsten, R.G. Deen, W. Goetz, C. Hardgrove, D.E. Harker, K.E. Herkenhoff, J.A. Hurowitz, L. Jandura, M.R. Kennedy, G. Kocurek, G.M. Krezoski, S.R. Kuhn, D. Limonadi, L. Lipkaman, M.B. Madsen, T.S. Olson, M.L. Robinson, S.K. Rowland, D.M. Rubin, C. Seybold, J. Schieber, M. Schmidt, D.Y. Sumner, V.V. Tompkins, J.K.V. Beek, T.V. Beek, J. Geophys. Res., Planets 118, 2338 (2013). https://doi.org/10.1002/ 2013JE004426

M.E. Minitti, K.S. Edgett, R.A. Yingst, P.G. Conrad, M. Fisk, C.J. Hardgrove, K.E. Herkenhoff, L.C. Kah, M.R. Kennedy, G.M. Krezoski, M.T. Lemmon, L. Lipkaman, S.R. Kuhn, M.L. Robinson, V.V. Tompkins, A. Treiman, K.H. Williford, MAHLI after dark: Nighttime Mars Hand Lens Imager observations under LED illumination, in Lunar Planet. Sci. Conf., vol. 45 (Lunar and Planetary Institute, Houston, Texas, 2014), p. 2029.

S.L. Murchie, J.F. Mustard, B.L. Ehlmann, R.E. Milliken, J.L. Bishop, N.K. McKeown, E.Z. Noe Dobrea, F.P. Seelos, D.L. Buczkowski, S.M. Wiseman, R.E. Arvidson, J.J. Wray, G. Swayze, R.N. Clark, D.J. Des Marais, A.S. McEwen, J.P. Bibring, J. Geophys. Res., Planets 114, 1-30 (2009). https://doi.org/10. 1029/2009JE003342

R.C. Moeller, L. Jandura, K. Rosette et al., The Sampling and Caching Subsystem (SCS) for the scientific exploration of Jezero crater by the Mars 2020 Perseverance rover. Space Sci. Rev. 217, 5 (2021). https:// doi.org/10.1007/s11214-020-00783-7

J.E. Moores, A.C. Schuerger, UV degradation of accreted organics on Mars: IDP longevity, surface reservoir of organics, and relevance to the detection of methane in the atmosphere. Journal of Geophysical Research: Planets 117 (2012). https://doi.org/10.1029/2012JE004060

J.F. Mustard, S.L. Murchie, S.M. Pelkey, B.L. Ehlmann, R.E. Milliken, J.A. Grant, J.-P. Bibring, F. Poulet, J. Bishop, E. Noe Dobrea, L. Roach, F. Seelos, R.E. Arvidson, S. Wiseman, R. Green, C. Hash, D. Humm, E. Malaret, J.A. McGovern, K. Seelos, T. Clancy, R. Clark, D.D. Marais, N. Izenberg, A. Knudson, Y. Langevin, T. Martin, P. McGuire, R. Morris, M. Robinson, T. Roush, M. Smith, G. Swayze, H. Taylor, T. Titus, M. Wolff, Nature 454(7202), 305-309 (2008). https://doi.org/10.1038/nature07097

L. Nagli, M. Gaft, Y. Fleger, M. Rosenbluh, Absolute Raman cross-sections of some explosives: Trend to UV. Opt. Mater. 30, 1747-1754 (2008). https://doi.org/10.1016/j.optmat.2007.11.019

NASA, Explore Science 2020-2024: A vision for science excellence (2020). https://science.nasa.gov/sciencepink/s3fs-public/atoms/files/2020-2024_Science-TAGGED.pdf

W. Nelson, R. Manoharan, J. Sperry, UV resonance Raman studies of bacteria. Appl. Spectrosc. Rev. 27(1), 67-124 (1992). https://doi.org/10.1080/05704929208018270

D.E. Osterbrock, S. Veilleux, Faint emission-lines in the spectrum of the orion nebula and the abundances of some of the rarer elements. Astrophys. J. 389(1), 305-324 (1992). https://doi.org/10.1086/171206

M.J. Pelletier, Appl. Spectrosc. 57, 20A (2003). https://doi.org/10.1080/13693780500338761

J.T. Perron, J.X. Mitrovica, M. Manga, I. Matsuyama, M.A. Richards, Evidence for an ancient Martian ocean in the topography of deformed shorelines. Nature 447(7146), 840-843 (2007). https://doi.org/10.1038/ nature 05873 
J. Razzell Hollis, Understanding morphology and photostability of organic solar cells via advanced structural probes. PhD Thesis, Imperial College London (2016). Available at: https://spiral.imperial.ac.uk/handle/ 10044/1/32271

J. Razzell Hollis, S. Ireland, W. Abbey, R. Bhartia, L.W. Beegle, Deep-ultraviolet Raman spectra of Marsrelevant evaporite minerals under $248.6 \mathrm{~nm}$ excitation. Icarus 351, 113969 (2020a). https://doi.org/10. 1016/j.icarus.2020.113969

J. Razzell Hollis, D. Rheingold, R. Bhartia, L. Beegle, An optical model for quantitative Raman microspectroscopy. Appl. Spectrosc. 74, 684-700 (2020b). https://doi.org/10.1177/0003702819895299

J. Razzell Hollis, T. Fornaro, W. Rapin, J. Wade, Á. Vicente-Retortillo, A. Steele, R. Bhartia, L.W. Beegle, Detection and degradation of adenosine monophosphate in perchlorate-spiked Martian regolith analog, by deep-UV spectroscopy. Astrobiology (2020c, in press)

F. Remondino, A. Rizzi, S. Girardi, F.M. Petti, M. Avanzini, 3D ichnology-Recovering digital 3D models of dinosaur footprints. Photogramm. Rec. 25(131), 266-282 (2010). https://doi.org/10.1111/j.1477-9730. 2010.00587.x

F. Rivera-Hernández, D.Y. Sumner, N. Mangold, S.G. Banham, K.S. Edgett, C.M. Fedo, S. Gupta, S. Gwizd, E. Heydari, S. Maurice, M. Nachon, H. Newsom, J. Schieber, K. Stack-Morgan, N. Stein, R.C. Wiens, Grain size variations in the Murray formation: Stratigraphic evidence for changing depositional environments in Gale crater, Mars. J. Geophys. Res., Planets 125, e2019JE006230 (2020). https://doi.org/10.1029/2019JE006230

M. Robinson, C. Collins, P. Leger, J. Carsten, V. Tompkins, F. Hartman, J. Yen, In-situ operations and planning for the Mars Science Laboratory robotic arm: The first 200 sols, in Proc. IEEE Internat. Conf. System of Systems Eng., vol. 8 (2013), pp. 153-158. https://doi.org/10.1109/SYSoSE.2013.6575259

J.D. Rodrigues, Defining, mapping and assessing deterioration patterns in stone conservation projects. J. Cult. Herit. 16, 267-275 (2015). https://doi.org/10.1016/j.culher.2014.06.007

J.A. Rodriguez-Manfredi, M. de la Torre Juárez, A. Alonso et al., The Mars Environmental Dynamics Analyzer, MEDA. A suite of environmental sensors for the Mars 2020 mission. Space Sci. Rev. 217, 48 (2021). https://doi.org/10.1007/s11214-021-00816-9

S.W. Ruff, K.A. Campbell, M.J. Van Kranendonk, M.S. Rice, J.D. Farmer, The case for ancient hot spring in Gusev crater, Mars. Astrobiology 20, 475-499 (2020). https://doi.org/10.1089/ast.2019.2044

E. Salas, R. Bhartia, L. Anderson, W. Hug, R. Reid, G. Iturrino, K. Edwards, In situ detection of microbial life in the deep biosphere in igneous ocean crust. Front. Microbiol. 6, 1260 (2015). https://doi.org/10. 3389/fmicb.2015.01260

H.M. Sapers, J. Razzell Hollis, R. Bhartia, L.W. Beegle, V.J. Orphan, J.P. Amend, Front. Microbiol. 10, 679 (2019). https://doi.org/10.3389/fmicb.2019.00679

E.L. Schock, M.D. Schulte, Organic synthesis during fluid mixing in hydrothermal systems. J. Geophys. Res., Planets 103(E12), 28513-28527 (1998). https://doi.org/10.1029/98JE02142

S.C. Schon, J.W. Head, C.I. Fassett, An overfilled lacustrine system and progradational delta in Jezero crater, Mars: Implications for Noachian climate. Planet. Space Sci. 67, 28-45 (2012). https://doi.org/10.1016/ j.pss.2012.02.003

M.A. Sephton, I.P. Wright, I. Gilmour, J.W. De Leeuw, M.M. Grady, C.T. Pillinger, High molecular weight organic matter in Martian meteorites. Planet. Space Sci. 50(7-8), 711-716 (2002). https://doi.org/10. 1016/S0032-0633(02)00053-3.

K.L. Siebach, M.B. Baker, J.P. Grotzinger, S.M. McLennan, R. Gellert, L.M. Thompson, J.A. Hurowitz, J. Geophys. Res., Planets 122, 295 (2017). https://doi.org/10.1002/2016JE005195

S.W. Squyres, O. Aharonson, B.C. Clark, B.A. Cohen, L. Crumpler, P.A. de Souza, W.H. Farrand, R. Gellert, J. Grant, J.P. Grotzinger, A.F.C. Haldemann, J.R. Johnson, G. Klingelhöfer, K.W. Lewis, R. Li, T. McCoy, A.S. McEwen, H.Y. McSween, D.W. Ming, J.M. Moore, R.V. Morris, T.J. Parker, J.W. Rice Jr., S. Ruff, M. Schmidt, C. Schröder, L.A. Soderblom, A. Yen, Pyroclastic activity at Home Plate in Gusev crater, Mars. Science 316, 738-742 (2007). https://doi.org/10.1126/science.1139045

K.M. Stack, N.R. Williams, F. Calef et al., Photogeologic map of the perseverance rover field site in Jezero crater constructed by the Mars 2020 science team. Space Sci. Rev. 216, 127 (2020). https://doi.org/10. 1007/s11214-020-00739-X

A. Steele, M. Fries, H.E.S. Amundsen, B. Mysen, M. Fogel, M. Schwitzer, N. Borter, A comprehensive imaging and Raman spectroscopy study of ALH84001 and a terrestrial analoguefrom Svalbard, in Abstracts of Papers, 37th Lunar and Planetary Science Conf., Abstract 2096, TX, USA (2006)

A. Steele, F.M. McCubbin, M.D. Fries, D.C. Golden, D.W. Ming, L.G. Benning, Graphite in the Martian meteorite Allan Hills 84001. Am. Mineral. 97, 1256-1259 (2012a). https://doi.org/10.2138/am.2012. 4148

A. Steele, F.M. McCubbin, M. Fries, L. Kater, N.Z. Boctor, M.L. Fogel, P.G. Conrad, M. Glamoclija, M. Spencer, A.L. Morrow, M.R. Hammond, R.N. Zare, E. Vicenzi, S. Siljestrom, R. Bowden, C.D.K. Herd, B.O. Mysen, S.B. Shirey, H.E.F. Amundsen, A.H. Treiman, E.S. Bullock, A.J.T. Jull, A reduced organic 
carbon component in Martian basalts. Science 337, 212-215 (2012b). https://doi.org/10.1126/science. 1220715

A. Steele, F. McCubbin, L.G. Benning, S. SiljestrÖem, G. Cody, Y. Goreva, E.H. Hauri, J. Wang, A.L.D. Kilcoyne, C. Grady, M. Smith, C. Freissinet, D.P. Glavin, A.S. Burton, M.D. Fries, J.D.R. Blanco, M. Glamoclija, K.L. Rogers, S. Mikhail, J. Dworkin, Organic carbon inventory of the Tissint meteorite, in 44th Lunar and Planetary Science Conference, Held March 18-22, 2013 in the Woodlands, Texas. LPI Contribution No. 2854 (2016)

J.C. Stern, B. Sutter, C. Freissinet, R. Navarro-González, C.P. McKay, P. Douglas Archer, A. Buch, A.E. Brunner, P. Coll, J.L. Eigenbrode, A.G. Fairen, H.B. Franz, D.P. Glavin, S. Kashyap, A.C. McAdam, D.W. Ming, A. Steele, C. Szopa, J.J. Wray, F.J. Martín-Torres, M. Zorzano, P.G. Conrad, P.R. Mahaffy, Proc. Natl. Acad. Sci. USA 112(14), 4245-4250 (2015). https://doi.org/10.1073/pnas.1420932112

C.R. Stoker, M.A. Bullock, Organic degradation under simulated Martian conditions. J. Geophys. Res., Planets 102(E5), 10881-10888 (1997). https://doi.org/10.1029/97JE00667

M. Storrie-Lombardi, W. Hug, G. McDonald, A. Tsapin, K. Nealson, Hollow cathode ion lasers for deep ultraviolet Raman spectroscopy and fluorescence imaging. Rev. Sci. Instrum. 72, 4452 (2001). https:// doi.org/10.1063/1.1369627

D.A.V. Stow, Sedimentary Rocks in the Field: A Colour Guide (Manson Publishing, London, 2005).

R.E. Summons, J.P. Amend, D. Bish, R. Buick, G.D. Cody, D.J. Des Marais, G. Dromart, J.L. Eigenbrode, A.H. Knoll, D.Y. Sumner, Astrobiology 11, 157 (2011). https://doi.org/10.1089/ast.2010.0506

V.Z. Sun, K.M. Stack, L.C. Kah, L. Thompson, W. Fischer, A.J. Williams, S.S. Johnson, R.C. Wiens, R.E. Kronyak, M. Nachon, C.H. House, S. VanBommel, Icarus 321, 866 (2019). https://doi.org/10.1016/j. icarus.2018.12.030

N. Tarcea, M. Harz, P. Rösch, T. Frosch, M. Schmitt, H. Thiele, R. Hochleitner, J. Popp, Spectrochim. Acta, Part A, Mol. Biomol. Spectrosc. 68, 1029 (2007). https://doi.org/10.1016/j.saa.2007.06.051

I.L. ten Kate, J.R.C. Garry, Z. Peeters, R.d. Quinn, B. Foing, P. Ehrenfreund, Amino acid photostability on the Martian surface. Meteorit. Planet. Sci. 40(8), 1185-1193 (2005). https://doi.org/10.1111/j.19455100.2005.tb00183.x

I.L. ten Kate, J.R.C. Garry, Z. Peeters, B. Foing, P. Ehrenfreund, The effects of Martian near surface conditions on the photochemistryof amino acids. Planet. Space Sci. 54, 296-302 (2006). https:// doi.org/10.1016/j.pss.2005.12.002

A. Toyama, N. Hanada, J. Ono, E. Yoshimitsu, H. Takeuchi, J. Raman Spectrosc. 30, 623 (1999). https:// doi.org/10.1002/jrs.1324

M. Trulson, R. Mathies, Raman cross section measurements in the visible and ultraviolet using an integrating cavity: Application to benzene, cyclohexane, and cacodylate. J. Chem. Phys. 84(4), 2068 (1986). https:// doi.org/10.1063/1.450415

D. Tuschel, A. Mikhonin, B. Lemoff, S. Asher, Deep ultraviolet resonance Raman excitation enables explosives detection. Appl. Spectrosc. 64(4), 425-432 (2010). https://doi.org/10.1366/000370210791114194

K. Uckert, R. Bhartia, J. Michel, A semi-autonomous method to detect cosmic rays in Raman hyperspectral data sets. Appl. Spectrosc. 73(9), 1019-1027 (2019). https://doi.org/10.1177/0003702819850584

S.J. VanBommel, R. Gellert, J.A. Berger, L.M. Thompson, K.S. Edgett, M.J. McBride, M.E. Minitti, N.I. Boyd, J.L. Campbell, Modeling and mitigation of sample relief effects applied to chemistry measurements by the Mars Science Laboratory alpha particle X-ray spectrometer. X-Ray Spectrom. 46, 229-236 (2017). https://doi.org/10.1002/xrs.2755

R.C. Wiens, S. Maurice, S.H. Robinson et al., The SuperCam instrument suite on the NASA Mars 2020 rover: Body unit and combined system tests. Space Sci. Rev. 217, 4 (2021). https://doi.org/10.1007/s11214020-00777-5

S.C. Werner, The early martian evolution-Constraints from basin formation ages. Icarus 195, 45-60 (2008). https://doi.org/10.1016/j.icarus.2007.12.008

K.H. Williford, K.A. Farley, K.M. Stack, A.C. Allwood, D. Beaty, L.W. Beegle, R. Bhartia, A.J. Brown, M. de la Torre Juarez, S.-E. Hamran, M.H. Hecht, J.A. Hurowitz, J.A. Rodriguez-Manfredi, S. Maurice, S. Milkovich, R.C. Wiens, The NASA Mars 2020 rover mission and the search for extraterrestrial life, in From Habitability to Life on Mars, ed. by N.A. Cabrol, E.A. Grin, (Elsevier, Amsterdam, 2018), pp. 275-308. https://doi.org/10.1016/B978-0-12-809935-3.00010-4. Chap. 11

H. Woodward, Photography in geology. Geol. Mag. (Decade IV) 10(9), 408-412 (1903). https://doi.org/10. 1017/S0016756800179476

J.J. Wray, B.L. Ehlmann, Geology of possible Martian methane source regions. Planet. Space Sci. 59(2-3), 196-202 (2011). https://doi.org/10.1016/j.pss.2010.05.006

Q. Wu, T. Hamilton, W.H. Nelson, S. Elliott, J.F. Sperry, M. Wu, Anal. Chem. 73, 3432 (2001). https:// doi.org/10.1021/ac001268b

Z. Wu, C. Zhang, P.C. Stair, Catal. Today 113, 40 (2006). https://doi.org/10.1016/j.cattod.2005.11.077 
R.A. Yingst, K.S. Edgett, M.R. Kennedy, G.M. Krezoski, M.J. McBride, M.E. Minitti, M.A. Ravine, R.M.E. Williams, MAHLI on Mars: Lessons learned operating a geoscience camera on a landed payload robotic arm. Geosci. Instrum. Method. Data Syst. 5, 205-217 (2016). https://doi.org/10.5194/gi-5-205-2016 